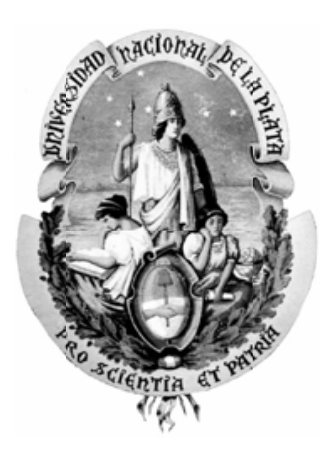

\title{
Transferencia de calor durante la congelación, el almacenamiento y la descongelación de alimentos
}

Viviana Olga Salvadori

Director: Dr. Rodolfo H. Mascheroni

Tesis presentada para optar al grado de DOCTOR EN INGENIERÍA 
INDICE

Capítulo I. INTRODUCCION

1.1. Presentación del tema

1.2. Importancia económica de la producción de alimentos congel ados en Argentina 1.2.1. Productos cárneos

1.2.2. Pescados y mariscos

1.2.3. Frutas y hortalizas

1.3. Descripción de objetivos y contenidos

Capítulo II. PREDICCION DE TIEMPOSDE CONGELACION DE ALIMENTOS

2.1. Introducción

2.2. El proceso decongelación

2.3. Métodos de predicción

2.3.1. Soluciones exactas

2.3.2. Soluciones aproximadas

2.4. Métodos de predicción desarrollados

2.4.1. Congelación simétrica

2.4.2. Congelación asimétrica

2.4.3. Resultados

2.4.3.1. Resultados de congelación simétrica

2.4.3.2. Resultados de congelación asimétrica

2.5. Conclusiones

Capítulo III. PREDICCION DE TIEMPOS DE DESCONGELACION DE ALIMENTOS

3.1. Introducción

3.2. El proceso de descongelación

3.3. Métodos de predicción

3.3.1. Soluciones exactas

3.3.2. Soluciones aproximadas

3.4. Métodos de predicción desarrollados

3.4.1. Descongelación simétrica

3.4.2. Resultados

3.5. Conclusiones 
Capítulo IV. ALMACENAMIENTO

4.1. Introducción

4.2. Funciones de transferencia

4.2.1. Formulación matemática

4.2.2. Equipo experimental

4.2.2.1. Cámara experimental de al macenamiento

4.2.2.2. Estibas

4.4.2.2.1. Tarima de cajas

4.4.2.2.2. Estibas de baldes

4.2.3. Descripción delas medidas experimentales

4.2.4. Cálculo de los coeficientes de la función de transferencia

4.2.4.1. Coeficientes experimentales

4.2.4.2. Coeficientes numéricos

4.2.5. Resultados: Predicción de respuestas térmicas frente a distintas perturbaciones.

4.3. Pérdida decalidad

Capítulo V. CON CLUSIONES GENERALES

NOMENCLATURA

REFERENCIASBIBLIOGRAFICAS 
CAPITULO I

INTRODUCCION 


\subsection{PRESENTACION DEL TEMA}

Dado el notable crecimiento que ha experimentado la población mundial en los últimos años, a un ritmo del 1,6\% anual (Mattarolo, 1990), es imprescindible estudiar todos los aspectos relacionados con la alimentación de la misma. Entre ellos merecen destacarse:

A.- Incremento de la producción total de alimentos, que puede lograrse aumentando el área cultivada; utilizando semillas genéticamente mejoradas; criando el ganado a establo, con alimento balanceado; y explotando mejor los recursos acuáticos.

B.- Mejoras en las tecnologías de conservación y procesamiento de modo de reducir al mínimo las pérdidas por deterioro (en algunos países en desarrollo estas últimas al canzan hasta el 50\% de la cosecha total).

C.- Mejoras en los sistemas de distribución de alimentos.

En el presente trabajo de tesis se analizarán distintos aspectos tecnológicos vinculados con la conservación de alimentos mediante congelación, directamente relacionados con los items B y C, con vistas a la mejora del diseño de equipos, aumento de la producción, disminución de los costos y mantenimiento de la calidad delos productos congelados.

En relación con estos aspectos los beneficios de la refrigeración en la conservación de alimentos se conocen desde hace varios siglos, si bien la utilización racional de las bajas temperaturas en la preservación comenzó hace cien años, como consecuencia del progreso tecnológico en los sistemas de refrigeración mecánica.

En la actualidad el impacto de la refrigeración es enorme, nuestro sistema de vida no puede ser imaginado sin el soporte de una cadena de frío. Como ejemplo podemos mencionar que el $40 \%$ de la producción mundial de alimentos, aproximadamente 1700 millones de tn, necesita refrigeración para evitar su deterioro. También juega un papel importante en los sistemas de transporte de 
alimentos, especialmente cuando se deben recorrer largas distancias entre los centros de procesamiento y consumo.

Las principales causas del deterioro de los alimentos son el crecimiento de microorganismos y las reacciones químicas, enzimáticas o no. Es sabido que el crecimiento microbiano puede ser drásticamente reducido disminuyendo la actividad acuosa. Uno de los primeros métodos en utilizarse para disminuir la actividad acuosa, eliminando el agua, fue el secado. Posteriormente, con el desarrollo de la microbiología, se perfeccionaron las técnicas de pasteurización y esterilización. La congelación no elimina totalmente los microorganismos vivos, sino que evita su multiplicación al eliminar del medio el agua en estado líquido. Por otro lado la gran mayoría de las reacciones químicas de deterioro son fuertemente dependientes de la temperatura, reduciéndose notablemente su velocidad con la disminución de esta variable, lo que se traduce en un aumento de la vida útil del alimento.

En la actualidad, si bien en varias situaciones cada uno de los diferentes métodos de preservación de alimentos presenta ventajas técnicas y económicas, se ha comprobado que los métodos de refrigeración, tanto el enfriamiento como la congelación, ofrecen las mayores posibilidades para mantener la calidad original del alimento fresco, presentando también ventajas desde el punto de vista económico para operaciones a gran escala. Si además se presta atención a los requerimientos de los consumidores, que día a día reclaman alimentos más sanos, de buena calidad, y con el menor tratamiento y/ o agregado posible de aditivos, la congelación resulta el mejor método para mantener durante un período de almacenamiento prolongado las características organolépticas del alimento fresco.

La congelación se practica con distintos fines:

- Prolongar la vida útil de alimentos altamente perecederos, que posteriormente son introducidos al mercado descongelados, y consumidos como "productos frescos". Esta operatoria permite absorber las variaciones estacionales de la oferta, como ejemplo podemos citar frutillas, espárragos, etc.

- Producir alimentos procesados como hamburguesas, productos de carne, Cap. $1-3$ 
pollo o pescado empanados, platos preparados listos para calentar y servir, etc. Estos productos día a día cuentan con mayor número de consumidores, en parte debido al ritmo de vida actual en las grandes ciudades, donde cada vez se reduce más el tiempo disponible para las tareas hogareñas.

- Congelar materias primas o productos semiprocesados que son almacenados para posteriormente ser descongelados y elaborados.

\subsection{IMPORTANCIA ECONOMICA DE LA PRODUCCION DE ALIMENTOS CONGELADOS EN ARGENTINA}

Argentina presenta, por sus características de suelo y clima tan variadas, condiciones óptimas para ser un país proveedor de alimentos.

Durante mucho tiempo y debido a la gran extensión dedicada exclusivamente a la actividad agropecuaria, con una altísima producción de cereales, especialmente trigo, nuestro país era reconocido como el "granero del mundo".

En la actualidad la situación del mercado internacional de materias primas agrícolas determina una baja generalizada del precio de las mismas, por lo que es imprescindible una reconversión del sector productivo para ofrecer al mercado internacional productos alimenticios de mayor valor agregado.

Si se atiende a la demanda internacional, se observa en los principales países importadores de alimentos (Estados Unidos, Alemania, Francia, Japón) una tendencia hacia los productos congelados en detrimento de otros productos procesados como enlatados y conservas.

A continuación detallamos la producción actual de alimentos congelados en Argentina, considerando tres rubros por separado:

1.2.1. Productos cárneos.

1.2.2. Pescados y mariscos.

1.2.3. Frutas y hortalizas. 


\subsubsection{PRODUCTOS CARNEOS}

Según un informe de D.I.E.P.B.A. (1991b), Argentina cuenta con 110 frigoríficos de los cuales 27 exportan más del 30\% de la faena. La faena total en el país en el año 1990 alcanzó a 2648800 tn.

El consumo interno, si bien es alto (el promedio en los últimos años es de $75.35 \mathrm{~kg}$ de carne vacuna y $6.55 \mathrm{~kg}$ de carne porcina per cápita por año) es mayoritariamente carne refrigerada o procesada en el caso de la carne porcina. El consumo de congelado corresponde casi exclusivamente a hamburguesas y productos empanados.

La Tabla 1.1 detalla el volumen de las exportaciones argentinas de carnes, en miles de toneladas equivalentes de res con hueso y en miles de U $\$ S$ corrientes, para el período 1988-1990. La Tabla 1.2 presenta el detalle de las exportaciones de carnes vacunas, y en la Tabla 1.3 se detallan los principales países de destino. 
Introducción

Tabla 1.1. Volumen de exportaciones argentinas de carnes (Fuente: D.I.E.P.B.A., 1991b).

\begin{tabular}{||l|r|r|r|r|r|r||}
\hline \multirow{2}{*}{ Producto } & \multicolumn{2}{|c|}{1988} & \multicolumn{2}{|c|}{1989} & \multicolumn{2}{|c|}{1990} \\
\cline { 2 - 7 } & $\operatorname{tn} 10^{3}$ & $\mathrm{U} \$ \mathrm{~S} 10^{3}$ & tn $10^{3}$ & $\mathrm{U} \$ \mathrm{~S} 10^{3}$ & tn $10^{3}$ & \multicolumn{1}{|c|}{$\mathrm{U} \$ \mathrm{~S} 10^{3}$} \\
\hline \hline Carnes vacunas & 243 & 550053 & 299 & 667048 & 349 & 821635 \\
Carnes ovinas & 7 & 7788 & 8 & 9744 & 10 & 10993 \\
Carnes porcinas & 2 & 1413 & 4 & 3106 & 3 & 2723 \\
Carnes equinas & 40 & 42263 & 41 & 45655 & 36 & 45736 \\
Subproductos & 11 & 19319 & 11 & 16615 & 13 & 17444 \\
Total & $\mathbf{3 0 3}$ & $\mathbf{6 2 0 8 3 6}$ & $\mathbf{3 6 3}$ & $\mathbf{7 4 2 1 6 8}$ & $\mathbf{4 1 1}$ & $\mathbf{8 9 8 5 3 1}$ \\
\hline \hline
\end{tabular}

Tabla 1.2. Volumen de exportaciones de carnes vacunas

(Fuente: D.I.E.P.B.A., 1991b).

\begin{tabular}{||l|r|r|r|r|r|r||}
\hline \multirow{2}{*}{ Producto } & \multicolumn{2}{|c|}{1986} & \multicolumn{2}{|c|}{1987} & \multicolumn{2}{|c||}{1990} \\
\cline { 2 - 7 } & \multicolumn{1}{|c|}{ tn } & U\$S 103 & \multicolumn{1}{c|}{ tn } & U\$S 103 & \multicolumn{1}{c|}{ tn } & U\$S 103 \\
\hline \hline Cuartos & 7275 & 8275 & 3568 & 4100 & 10741 & 11807 \\
Cortes & 63402 & 176994 & 68214 & 261456 & 101475 & 193818 \\
Manuf. cong. & 6595 & 6265 & 2742 & 3450 & 4930 & 4451 \\
Enlatada & 28777 & 58644 & 43839 & 107641 & 48356 & 108223 \\
Coc. y cong. & 23236 & 74980 & 30600 & 105946 & 35759 & 107501 \\
Otros & 8016 & 17623 & 5791 & 14585 & 8482 & 20583 \\
Total & $\mathbf{1 3 7 3 0 1}$ & $\mathbf{3 4 2 7 8 1}$ & $\mathbf{1 5 4 7 5 4}$ & $\mathbf{4 9 7 1 7 8}$ & $\mathbf{2 0 9 7 4 3}$ & $\mathbf{4 4 6 3 8 3}$ \\
\hline \hline
\end{tabular}

Notas: -La Junta Nacional de Carnes se encuentra reconstruyendo la información de los años 1988 y 1989, por lo cual se ha incluído la correspondiente a los años 1986 y 1987.

- Los items "Cuartos" y "Cortes" corresponden a producto enfriado (aproximadamenteel 30\%) y congelado (el 70\% restante).

Tabla 1.3. Exportaciones de carnes vacunas según países de destino

(Fuente: D.I.E.P.B.A., 1991b). 
Introducción

\begin{tabular}{|c|c|c|c|c|c|c|}
\hline \multirow{2}{*}{$\begin{array}{l}\text { ¡Error! } \\
\text { M arcador no } \\
\text { definido.Produ } \\
\text { cto }\end{array}$} & \multicolumn{2}{|c|}{1988} & \multicolumn{2}{|c|}{1989} & \multicolumn{2}{|c|}{1990} \\
\hline & tn $10^{3}$ & U\$S $10^{3}$ & $\operatorname{tn} 10^{3}$ & U\$S $10^{3}$ & $\operatorname{tn} 10^{3}$ & U\$S $10^{3}$ \\
\hline Alemania & 34 & 195748 & 37 & 196446 & 34 & 177704 \\
\hline E.E.U.U. & 42 & 111934 & 42 & 115035 & 47 & 135313 \\
\hline Inglaterra & 21 & 39931 & 28 & 67218 & 32 & 73231 \\
\hline Italia & 14 & 26797 & 19 & 35190 & 22 & 47500 \\
\hline Resto & 132 & 175629 & 173 & 253159 & 214 & 387887 \\
\hline Total & 243 & 555039 & 299 & 667048 & 349 & 821635 \\
\hline
\end{tabular}

Estas estadísticas no incluyen a los productos que se congelan para consumo interno, principalmente como hamburguesas de distintas carnes, porciones empanadas, comidas preparadas en base a carnes, etc.

\subsubsection{PESCADOS Y MARISCOS}

La tabla 1.4 detalla la captura anual según las distintas especies, en el período 1988-1990. En promedio el 51.4\% se destinó para el consumo interno y el $48.6 \%$ se destina a exportación. En 1990 nuestro país contaba con 124 firmas exportadoras de productos pesqueros, las 10 empresas principales exportan el 56\% del total. Como puede observarse en la Tabla 1.5 el volumen exportado es mayoritariamente producto congelado. La Tabla 1.6 presenta los principales países de destino y los volumenes exportados a cada uno de ellos.

El consumo interno de productos congelados es muy pequeño. Está compuesto principalmente por filetes de merluza, camarones, langostinos y porciones de empanados y rebozados. 
Tabla 1.4. Captura anual por especie, en tn (Fuente: D.I.E.P.B.A., 1991a).

\begin{tabular}{||l|r|r|r||}
\hline \hline Especie & \multicolumn{1}{|c|}{1988} & 1989 & \multicolumn{1}{c|}{1990} \\
\hline \hline Merluza & 296026 & 294332 & 341042 \\
A badejo & 17307 & 20797 & 34096 \\
Otros pescados & 128682 & 121729 & 127485 \\
Total pescados & $\mathbf{4 4 2 0 1 5}$ & $\mathbf{4 3 6 8 5 8}$ & $\mathbf{5 0 2 6 2 3}$ \\
Calamar & 20777 & 23105 & 27603 \\
Otros moluscos & 1432 & 3452 & 4370 \\
Langostino & 17800 & 11680 & 9852 \\
Otros crustáceos & 586 & 429 & 493 \\
Total mariscos & $\mathbf{4 0 5 9 4}$ & $\mathbf{3 8 6 6 6}$ & $\mathbf{4 2 3 1 7}$ \\
Total & $\mathbf{4 8 2 6 0 9}$ & $\mathbf{4 7 5 5 2 4}$ & $\mathbf{5 4 4 9 4 1}$ \\
\hline \hline
\end{tabular}

Tabla 1.5. Volumen de exportaciones de productos pesqueros (Fuente: D.I.E.P.B.A., 1991a).

\begin{tabular}{||l|r|r|r|r|r|r||}
\hline \multirow{2}{*}{ Producto } & \multicolumn{2}{|c|}{1988} & \multicolumn{2}{|c|}{1989} & \multicolumn{2}{|c||}{1990} \\
\cline { 2 - 7 } & \multicolumn{1}{c|}{ tn } & $\mathrm{U} \$ \mathrm{~S} 10^{3}$ & \multicolumn{1}{c|}{ tn } & $\mathrm{U} \$ \mathrm{~S} 10^{3}$ & \multicolumn{1}{c|}{ tn } & \multicolumn{1}{c|}{$\mathrm{U} \$ \mathrm{~S} 10^{3}$} \\
\hline \hline Congelado & 194138 & 254283 & 203158 & 264286 & 235623 & 300527 \\
Enfriado & 10856 & 19260 & 14468 & 25072 & 12060 & 20514 \\
Otros & 6532 & 2478 & 11705 & 3773 & 7398 & 2712 \\
Total & $\mathbf{2 1 1 5 2 6}$ & $\mathbf{2 7 6 0 2 0}$ & $\mathbf{2 2 9 3 3 1}$ & $\mathbf{2 9 3 1 3 1}$ & $\mathbf{2 5 5 0 8 1}$ & $\mathbf{3 2 3 7 5 3}$ \\
\hline \hline
\end{tabular}


Tabla 1.6. Exportaciones, según países de destino (Fuente: D.I.E.P.B.A., 1991a)

\begin{tabular}{|l|r|r|r|r|r|r||}
\hline \multirow{2}{*}{ Producto } & \multicolumn{2}{|c|}{1987} & \multicolumn{2}{c|}{1988} & \multicolumn{2}{|c|}{1990} \\
\cline { 2 - 7 } & \multicolumn{1}{c|}{ tn } & U\$S $10^{3}$ & \multicolumn{1}{c|}{ tn } & U\$S 103 & \multicolumn{1}{c|}{ tn } & U\$S 103 \\
\hline \hline España & 49952 & 64565 & 34235 & 67985 & 46627 & 74463 \\
Italia & 32034 & 32365 & 21275 & 35641 & 28181 & 45325 \\
E.E.U.U. & 34589 & 49224 & 27478 & 41216 & 27800 & 45325 \\
Brasil & 19023 & 14065 & 20003 & 15303 & 46958 & 45325 \\
Francia & 11811 & 14854 & 8882 & 9764 & 8461 & 9713 \\
Japón & 13084 & 15756 & 16180 & 35765 & 19257 & 22663 \\
Alemania & 9303 & 11462 & 7589 & 9157 & 12764 & 16188 \\
Resto & 71403 & 65015 & 75884 & 61189 & 65033 & 64751 \\
Total & $\mathbf{2 4 1 1 9 9}$ & $\mathbf{2 6 7 3 0 6}$ & $\mathbf{2 1 1 5 2 6}$ & $\mathbf{2 7 6 0 2 0}$ & $\mathbf{2 5 5 0 8 1}$ & $\mathbf{3 2 3 7 5 3}$ \\
\hline
\end{tabular}

\subsubsection{FRUTAS Y HORTALIZAS}

En estos productos la proporción de la producción argentina que se congela aún es muy reducida, aunque en los últimos años se ha producido cierto incremento de la misma, instalándose varias plantas de congelación de arvejas, espárragos, maíz dulce, porotos, papas y zanahorias. La información existente se da en la Tabla 1.7.

Atendiendo a los principales países importadores (EEUU, Europa y Japón) que consumen aproximadamente 17000000 tn de productos hortifrutícolas congelados, queda claro que existe un mercado potencial al que habrá que llegar cumpliendo especialmente requisitos de calidad, por lo cual deben atenderse a todos los factores que determinan las características finales del producto congelado.

Tabla 1.7. Volumen de exportaciones de productos frutihortícolas congelados, en tn (Fuente: PROM EX, 1992b). 
Introducción

\begin{tabular}{|l|r|r|r||}
\hline \hline $\begin{array}{l}\text { iError! Marcador no } \\
\text { definido.Producto }\end{array}$ & 1989 & 1990 & \multicolumn{1}{|c|}{1991} \\
\hline \hline Maíz dulce & 3 & 2 & 1 \\
Choclo & 172 & 370 & 77 \\
Porotos & 136 & 152 & 178 \\
Arvejas & 126 & 153 & 137 \\
Fruta & 168 & 147 & 143 \\
Fruta d azúcar & 470 & 247 & 252 \\
Total & $\mathbf{1 0 7 5}$ & $\mathbf{1 0 7 1}$ & $\mathbf{6 8 8}$ \\
\hline \hline
\end{tabular}

N ota: Los valores del año 1991 corresponden al primer semestre.

Por otra parte el consumo interno también aporta un volumen en crecimiento principal menteen la franja de papas congeladas (prefritas o no), juliana, arvejas, choclo y espinaca.

\subsection{DESCRIPCION DE OBJETIVOS Y CONTENIDOS}

Como ya se ha mencionado, en el presente trabajo se estudian distintos aspectos relacionados con la congelación y el almacenamiento de productos congelados, con los siguientes objetivos:

- Optimizar el diseño de sistemas para la congelación o almacenamiento de alimentos a través de un mejor conocimiento de los fenómenos de transferencia de calor que tienen lugar en los mismos. Esto involucra un adecuado dimensionamiento de los equipos con el consiguiente ahorro en los costos de inversión.

- Desarrollar métodos simplificados y muy precisos de predicción de tiempos de congelación y descongelación, con lo que se contribuirá al objetivo anterior y, principalmente, a la operación eficiente de los equipos con los consiguientes ahorros de energía y costos de operación y mejora en la calidad de los productos procesados. 
- Estudiar el comportamiento térmico de diversas estibas de alimentos congelados frente a fluctuaciones de la temperatura del ambiente de almacenamiento y analizar los efectos de éstas sobre la calidad de los alimentos, para optimizar el diseño de los sistemas de almacenamiento y distribución.

Con estos fines se planificaron las investigaciones cuyos desarrollos y resultados se presentan en los capítulos siguientes, las mismas se reseñan a continuación:

La necesidad de operar eficientemente los equipos industriales de congelación (tales como lechos fluidizados, túneles continuos, congeladores a placas, etc.), para obtener productos de óptima calidad, requiere conocer adecuadamente todas las variables que afectan al proceso. Una de las variables más importante es el tiempo de congelación, ya que determina el tiempo de residencia de los alimentos en los equipos. Si esta variable es subdimensionada los productos abandonan el equipo de congelación a una temperatura mayor que la supuesta. Al ingresar a la cámara de almacenamiento se produce un incremento de la carga térmica ya que las cámaras están diseñadas para mantener los alimentos a una determinada temperatura y no para enfriarlos; además el tiempo que resta para alcanzar la temperatura final puede extenderse demasiado. Por el contrario un tiempo de congelación mayor que el necesario significa que se está desperdiciando energía en el equipo de congelación. En el capítulo II luego de realizar una exhaustiva revisión de los métodos conocidos de cálculo del tiempo de congelación (Cleland, 1990), y ante la falta de un método simple y preciso válido para un rango amplio de condiciones operativas, se ha desarrollado un método simplificado de predicción de tiempos de congelación de alimentos con transferencia de calor unidimensional, que considera condiciones de contorno simétricas y asimétricas.

Además de congelarse productos finales, en los últimos años se ha manifestado una tendencia a congelar materias primas o productos semiprocesados y almacenarlos hasta que llegue el momento del procesamiento final. En esta operatoria aparece como variable tecnológica el tiempo de descongelación. En el capítulo III se desarrolla un esquema similar al propuesto en el capítulo II para el cál culo de tiempos de descongelación de alimentos de formas regulares, válido en la mayoría de las situaciones encontradas en la práctica industrial. 
Como ya hemos mencionado, el objetivo de la congelación es retardar el deterioro de los alimentos. Generalmente los alimentos congelados son almacenados por un período prolongado; debe prestarse especial atención a las condiciones en que se realiza este almacenamiento para que el producto llegue al consumidor manteniendo su calidad original. La variable que más influye en el deterioro durante el almacenamiento es la temperatura, el deterioro es mayor si se producen fluctuaciones de la temperatura ambiental. En el capítulo IV se utilizan conceptos de funciones de transferencia para estudiar el comportamiento térmico de distintas estibas de alimentos congel ados frente a determinadas fluctuaciones de la temperatura del ambiente de al macenamiento. Se analiza también la influencia de fallas en el suministro de energía eléctrica o en el funcionamiento de los equipos de refrigeración en la evolución de la temperatura de las estibas almacenadas. Por último se describen los cambios en el alimento que suelen utilizarse como indicadores de la calidad y se realiza el cálculo del deterioro producido por diferentes historias térmicas.

El capítulo V presenta las conclusiones generales. 
CAPITULO II

\section{PREDICCION DE TIEM POS DE CONGELACION DE ALIMENTOS}




\subsection{INTRODUCCION}

La congelación es la tecnología de preservación de alimentos de mayor aplicación cuando el objetivo es lograr largos períodos de almacenamiento, manteniendo prácticamente inalteradas las características originales del alimento.

Al planificar adecuadamente un proceso de congelación, deberán considerarse varios aspectos para lograr un producto final de calidad óptima. Definidas las características del alimento (forma, composición, tamaño, empaque) y seleccionado el equipo a utilizar, determinando las condiciones operativas del mismo (temperatura del refrigerante, coeficiente de transferencia calórica, temperatura a la que ingresará el producto) importa principalmente poder calcular el tiempo de residencia del producto en el equipo de congelación, el que está determinado por las características del producto y por las condiciones operativas.

Aquí es evidente que es necesario contar con métodos de predicción del tiempo de congelación muy precisos ya que si dicho tiempo es subestimado el alimento abandonará el equipo de congelación parcialmente congelado y, por el contrario, si dicha variable es sobreestimada estaremos desaprovechando parte de la capacidad de operación del equipo y consumiendo energía en exceso, lo cual es una gran desventaja desde un punto de vista económico.

Antes de discutir las distintas metodologías existentes para calcular el tiempo de congelación, debemos especificar adecuadamente la mencionada variable ya que en la literatura encontramos diversas interpretaciones de la misma, en parte debido a que al finalizar la congelación la temperatura del alimento no es uniforme, razón por la cual el tiempo de congelación puede calcularse respecto de diversos puntos; y en parte debido a que distintos autores han tomado diferentes temperaturas como representativas del final de la congelación.

Como punto de referencia adoptaremos el centro térmico que se define como el punto de mayor temperatura al finalizar la congelación. En alimentos de forma regular (placa plana, cilindro infinito o finito, esfera, paquete prismático) y

Cap. 2-2 
cuando el fenómeno de transferencia de calor está gobernado por conducción y las condiciones operativas son simétricas, el centro térmico coincide con el centro geométrico de la pieza.

Como definición del tiempo de congelación adoptaremos la presentada por el IIR (1972): "El tiempo efectivo de congelación es el tiempo requerido para bajar la temperatura de su valor inicial a un valor dado en el centro térmico".

\subsection{EL PROCESO DE CONGELACION}

La congelación puede dividirse en tres etapas (a los efectos de su visualización, en realidad estas etapas se superponen parcialmente):

- Preenfriamiento: En este período el alimento se enfría desde su temperatura inicial $\mathrm{T}_{\mathrm{i}}$, que generalmente es mayor que $0^{\circ} \mathrm{C}$, hasta la temperatura de comienzo de cambio de fase.

- Cambio de fase: Este período está caracterizado por la nucleación y el crecimiento de cristales de hielo. El tamaño de los cristales dependerá de la velocidad de transferencia de calor.

La gran mayoría de los alimentos está constituida por una matriz de sólidos insolubles y una fase acuosa que contiene una cierta cantidad de sólidos disueltos. Debido a estos sólidos la congelación del agua no se produce a $0^{\circ} \mathrm{C}$ sino que el cambio de fase comienza a una temperatura menor $\mathrm{T}_{\mathrm{f}}$, la magnitud del descenso crioscópico es proporcional a la concentración inicial de sólidos. Al avanzar la congelación la concentración de sólidos en la fase sin congelar aumenta, razón por la cual la temperatura de cambio de fase continúa descendiendo obteniéndose un rango de temperaturas de cambio de fase. La curva de equilibrio del contenido de hielo vs. temperatura será particular para cada alimento pero presenta la forma general que se observa en la Figura 2.1. 


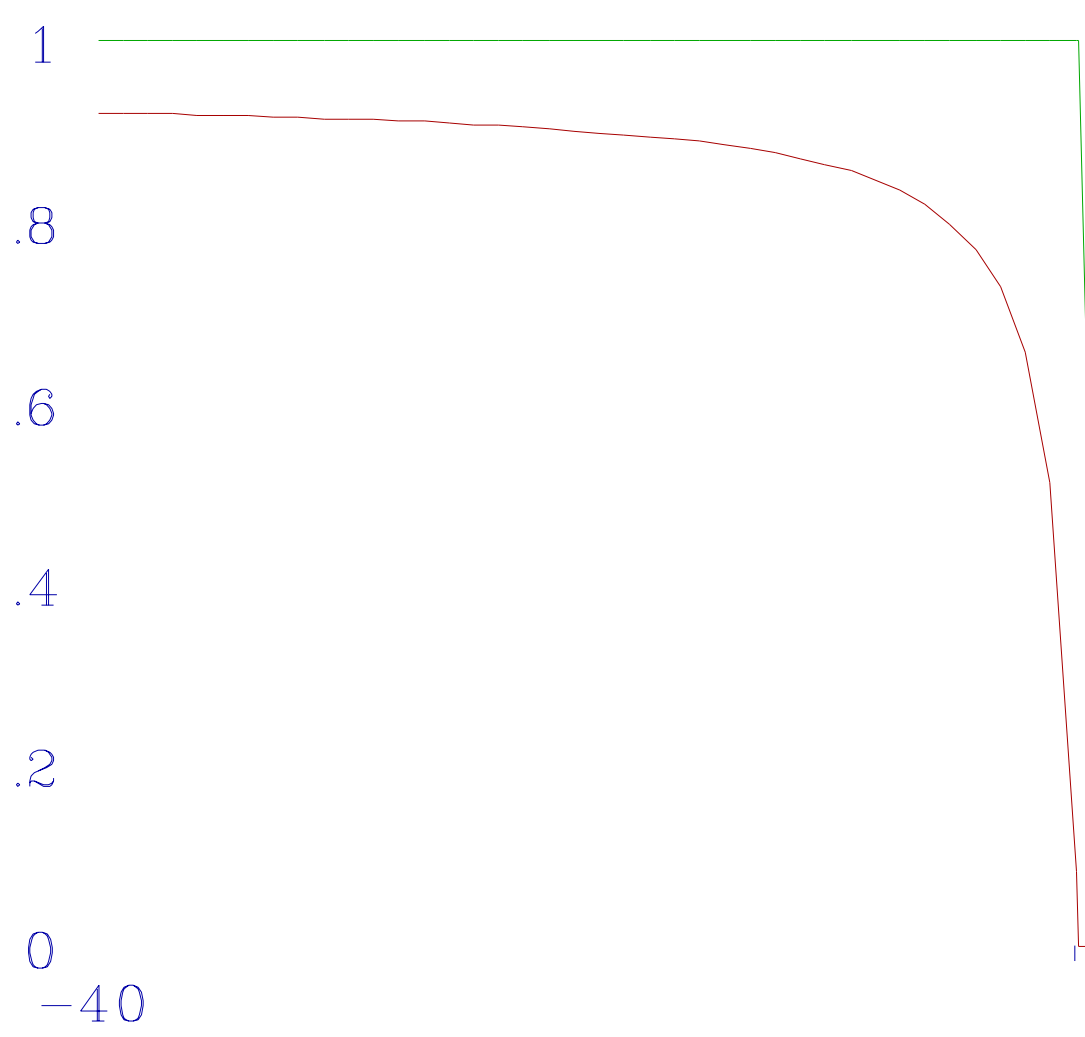

Figura 2.1. Contenido de hielo vs. temperatura, para agua y para un alimento.

Se encuentra además que aún a temperaturas muy bajas, por ejemplo $40^{\circ} \mathrm{C}$, una pequeña proporción del contenido inicial de agua permanece sin congelar, esta fracción se denomina "agua ligada". A los fines prácticos se considera que, para alimentos de alto contenido acuoso, la mayor parte del cambio de fase ocurre entre $\operatorname{Tf}_{\mathrm{f}}-5^{\circ} \mathrm{C}$.

- Atemperado: Esta última etapa consiste en enfriar el alimento ya congelado hasta la temperatura final $\mathrm{T}_{\mathrm{c}}$. Si bien no existen limitaciones para seleccionar $\mathrm{T}_{\mathrm{c}}$, los dos valores más utilizados en la práctica son -10 y $-18^{\circ} \mathrm{C}$.

\subsection{METODOS DE PREDICCION}

Los distintos métodos conocidos de cálculo del tiempo de congelación se basan en diferentes soluciones del balance diferencial de energía térmica, que 
simplificado para un alimento sólido (ver inciso 2.4.), resulta:

$$
\rho C_{p} \frac{\partial T}{\partial t}=\Delta(k \Delta T)
$$

donde $\mathrm{T}$ es la temperatura, $\mathrm{t}$ el tiempo, $\rho$ la densidad, $\mathrm{C}_{\mathrm{p}}$ el calor específico y $\mathrm{k}$ la conductividad térmica.

Los alimentos presentan distintas formas geométricas, regulares e irregulares. En el presente trabajo nos ocuparemos principalmente de las formas regulares, con transferencia de energía en una única dirección: placa plana infinita, cilindro infinito y esfera.

El cambio de fase del agua conlleva una variación importante de todas las propiedades físicas involucradas en el fenómeno de transferencia de calor. La conductividad térmica del hielo, por ejemplo, es 5 veces mayor que la conductividad térmica del agua líquida. Es de destacar que el cambio no sólo se da entre los estados inicial y final sino que es gradual y afecta a todo el proceso de congelación. Como ejemplo, en las Figuras 2.2, 2.3 y 2.4 se han graficado la variación con la temperatura de la densidad, el calor específico y la conductividad térmica de carne vacuna, respectivamente.

La condición inicial más frecuente es que el alimento se encuentra a una temperatura uniforme, $\mathrm{T}_{\mathrm{i}}$, por encima del punto de congelación.

En cuanto a las condiciones de contorno pueden presentarse cuatro variantes:

- La temperatura de la superficie es constante (conocida como condición de contorno de primer tipo). 


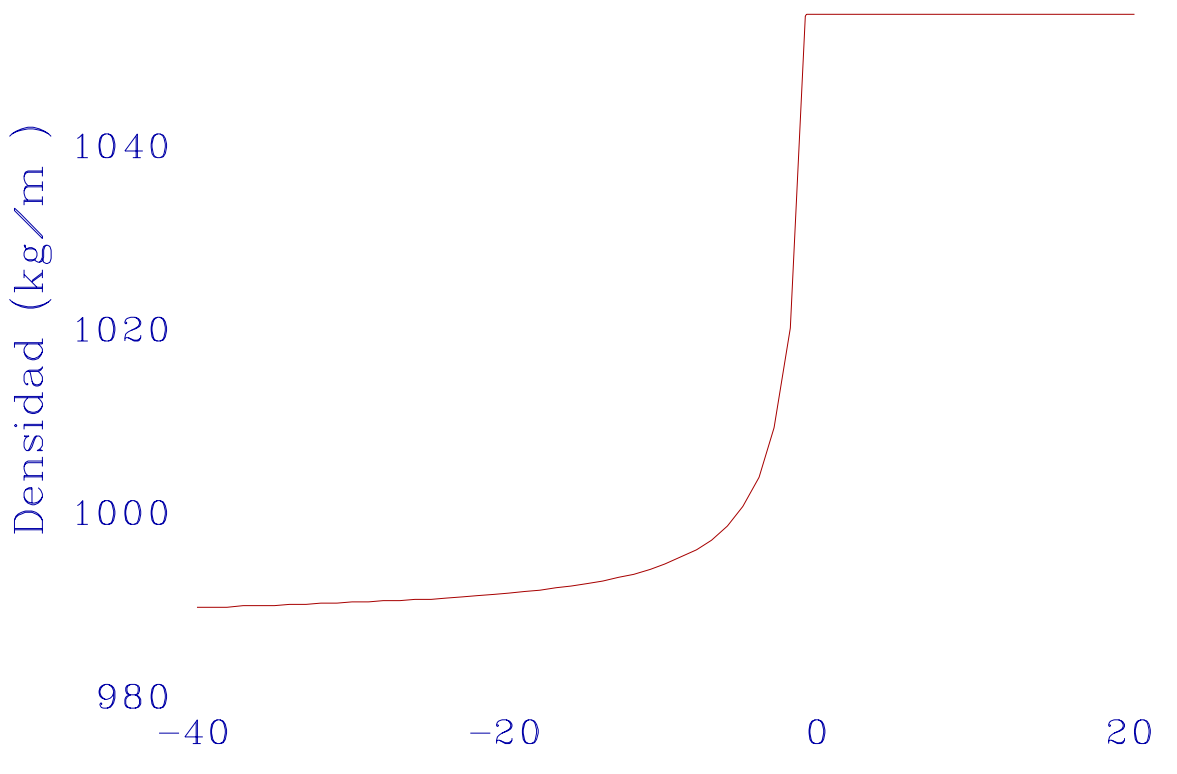

Figura 2.2. Densidad de carne en función de la temperatura.

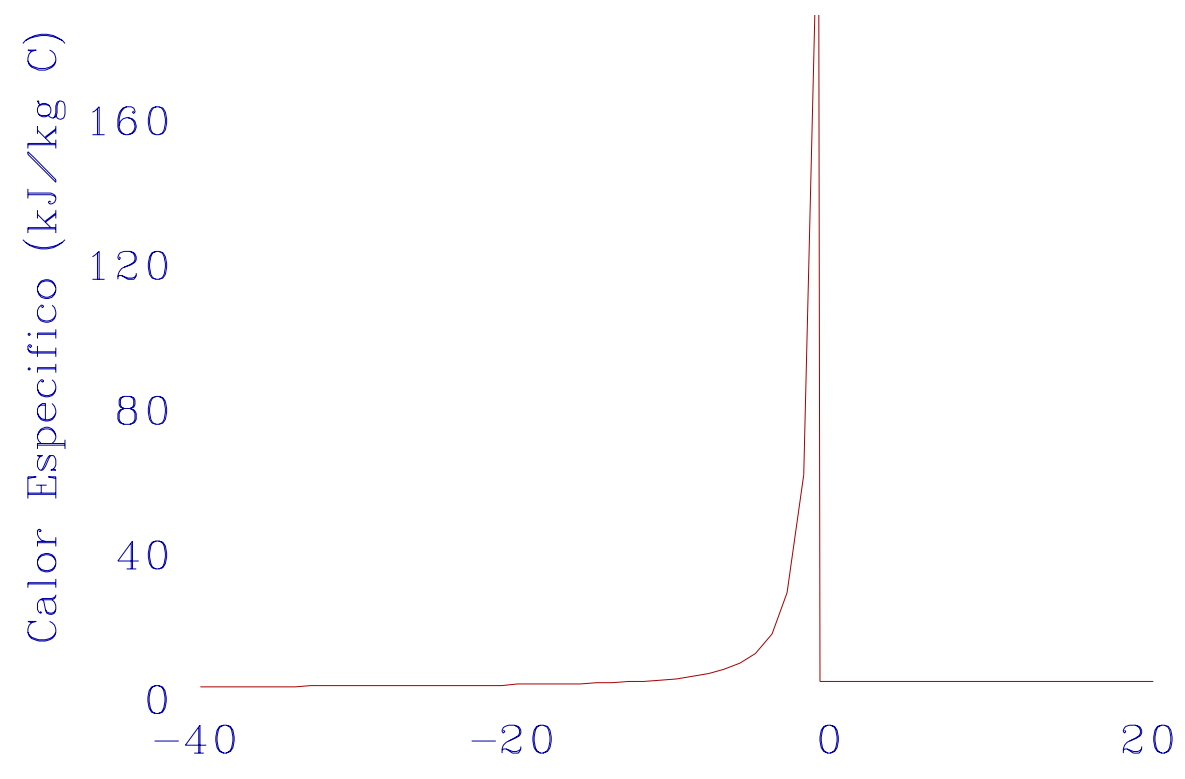

Figura 2.3. Calor específico de carne en función de la temperatura. 


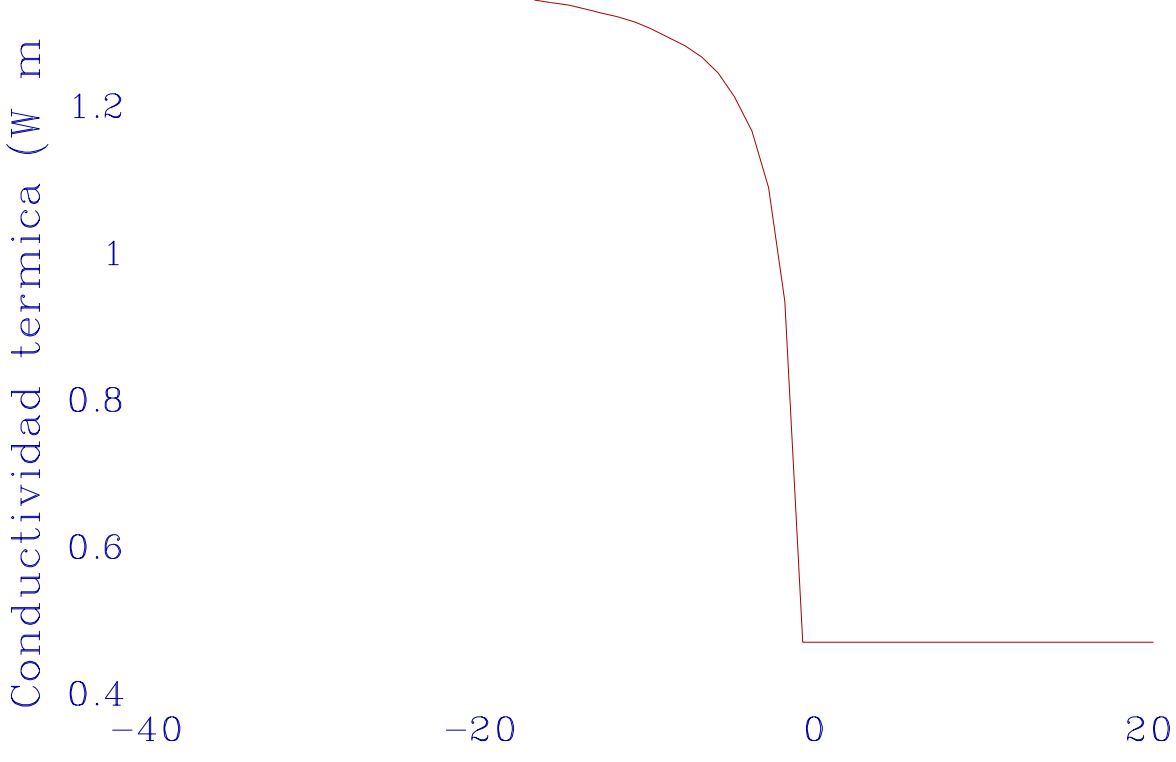

Figura 2.4. Conductividad térmica de carne en función de la temperatura.

- El flujo de calor en la superficie es constante y conocido (condición de contorno de segundo tipo).

- El flujo de calor en la superficie puede expresarse según $k(d T / d r)=h\left(T_{a}-T\right)$ conocida como "ley de enfriamiento" de Newton o condición convectiva (condición de contorno de tercer tipo, la más habitual en la congelación industrial).

- La temperatura de la superficie es una dada función del tiempo (condición de contorno de cuarto tipo).

Las soluciones conocidas del balance (2.1) pueden agruparse en dos grandes grupos:

\subsubsection{Soluciones "exactas".}

\subsubsection{Soluciones aproximadas.}




\subsubsection{SOLUCIONES EXACTAS}

Debido a que las propiedades termofísicas $\rho, C_{p}$ y $k$ dependen fuertemente de la temperatura, la ec. (2.1) no tiene una solución analítica exacta que contemple las condiciones más generales de la congelación.

La mayoría de las soluciones analíticas exactas conocidas, obtenidas para placa plana semiinfinita, corresponden a casos simplificados, por ejemplo consideran el caso más simple de frente móvil con temperatura de cambio de fase constante. Ninguna de ellas excepto la solución obtenida por Plank (que se describirá detalladamente en el inciso 2.3.2.) tiene en cuenta la condición de contorno de tercer tipo, por lo que no son aplicables a casos prácticos. Entre ellas podemos citar las soluciones de Stefan y de Neumann (Carslaw \& Jaeger, 1959) y Frederick \& Grief (1985).

La otra alternativa para encontrar una solución exacta de la ec. (2.1) es plantear un modelo numérico. Las características generales de los métodos numéricos se enumeran a continuación:

- No simplifican el balance diferencial, se introduce error al discretizar las derivadas, pero dicho error es minimizado tomando pequeños incrementos de espacio y tiempo.

- Calculan los perfiles de temperatura en función del tiempo además del tiempo total de proceso.

- Utilizan ecuaciones teóricas o valores experimentales para considerar la dependencia de las propiedades físicas con la temperatura.

- No tienen restricciones en cuanto al rango de condiciones operativas.

- Permiten analizar distintos tipos de condiciones de contorno, aún aquellas variables en el tiempo.

El modelo numérico puede plantearse utilizando diferencias finitas o elementos finitos. Se han propuesto modelos numéricos basados en la integración de una ecuación diferencial ordinaria que expresa la posición del frente móvil (Hayakawa y col. (1986)), pero estos modelos son menos realistas que los que se basan en el balance diferencial de energía discretizado por diferencias o elementos finitos. Cuando se tiene que elegir un modelo se recomienda adoptar el método

Cap. 2-8 
más simple, que genere menores errores en su implementación.

Además de los errores de truncación y redondeo, las otras fuentes de error de los métodos numéricos se encuentran en la forma de discretizar las condiciones de contorno y en la manera en que se expresa la funcionalidad que vincula las propiedades físicas con la temperatura (ambas fuentes de error son independientes del método utilizado).

Los esquemas de diferencias finitas son más simples, requieren menor disponibilidad de memoria y el tiempo de cómputo es menor que en los modelos de elementos finitos; dan resultados igualmente satisfactorios cuando se trabaja con formas regulares (placa plana, cilindro, esfera). Cuando la forma del alimento es irregular resulta más fácil plantear un esquema que utilice elementos finitos ya que las diferencias finitas necesitarán una grilla distorsionada que contemple el contorno del alimento. El estudio de la congelación de alimentos de formas irregulares cae fuera del alcance de este trabajo, razón por la cual sólo se analizarán los esquemas planteados con diferencias finitas.

En la literatura encontramos una gran variedad de modelos, sólo discutiremos los que han tenido mayor uso, y que han sido contrastados con valores experimentales. Para poder comparar los distintos esquemas, presentaremos brevemente cada uno de ellos.

Se considerará la congelación de una placa plana infinita, con transferencia de energía en una única dirección. La grilla de la Figura 2.5 indica como se discretiza el espacio (subíndice i) y el tiempo (supraíndice n); $\Delta \mathrm{r}$ es el incremento de espacio y $\Delta t$ el intervalo de tiempo.

El balance (2.1) llevado al caso de una placa plana es:

$$
\rho C_{p} \frac{\partial T}{\partial t}=\frac{\partial}{\partial r}\left(k \frac{\partial T}{\partial r}\right)
$$




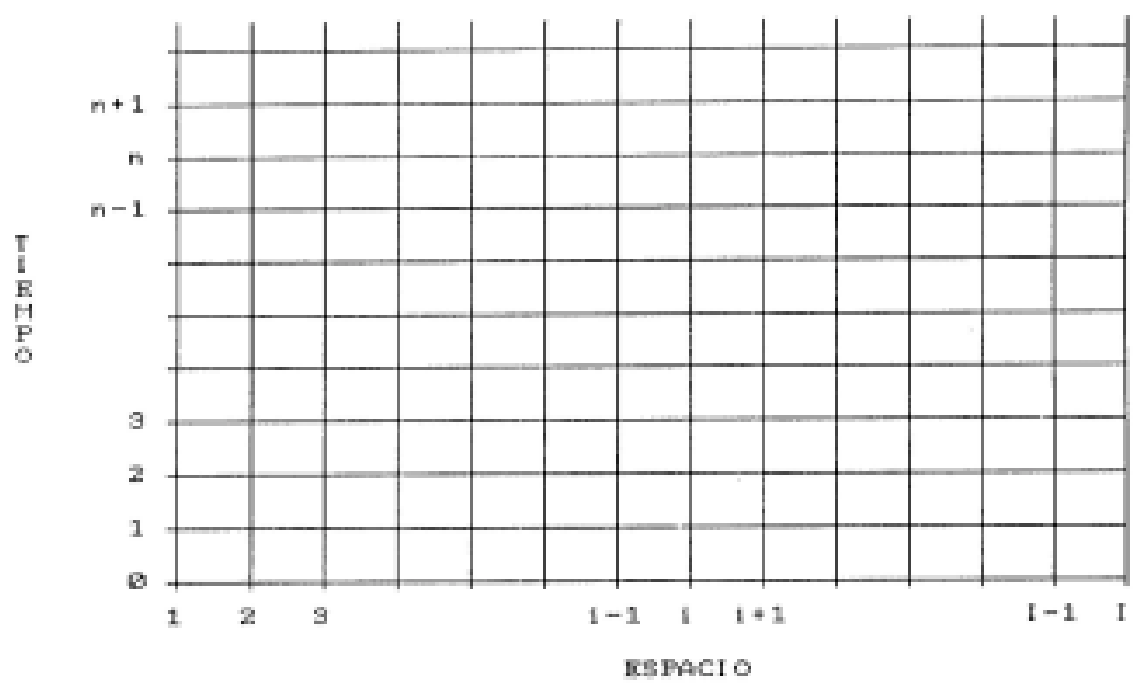

Figura 2.5. Grilla de discretización de espacio y tiempo.

Esquema explícito: La forma general del balance discretizado queda expresada en la ec. (2.3):

$$
\left(\rho C_{p}\right)_{i}^{n} \frac{T_{i}^{n+1}-T_{i}^{n}}{\Delta t}=\frac{1}{(\Delta r)^{2}}\left[k_{i+1 / 2}^{n}\left(T_{i+1}^{n}-T_{i}^{n}\right)-k_{i-1 / 2}^{n}\left(T_{i}^{n}-T_{i-1}^{n}\right)\right]
$$

Es evidente que la variable desconocida, $T_{i}^{n+1} 1$, se despeja fácilmente de la ec. (2.3).

La desventaja del metodo explícito es que para cumplir la condición de convergencia manteniendo una precisión adecuada se debe trabajar con incrementos de tiempo $\Delta t$ muy pequeños, lo cual origina mayores tiempos de ejecución de programa; aunque actualmente ésto ya no es una dificultad debido a la gran velocidad de cálculo de las computadoras modernas. Se debe trabajar con incrementos de tiempo pequeños también para minimizar el error que se comete al evaluar el producto $\rho C_{p}$ en el tiempo $n \Delta_{t}$ y no en el instante medio entre $n \Delta_{t} y$ $(\mathrm{n}+1) \Delta \mathrm{t}$.

Mascheroni \& Calvelo (1982) y Bazán \& Mascheroni (1984) han utilizado 
un esquema de diferencias finitas explícito para placa plana infinita, con propiedades de carne vacuna y ovina respectivamente, que ha sido contrastado con valores experimentales de tiempos de congelación.

De Michelis \& Calvelo (1982) utilizan un esquema similar para modelar la congelación de placa plana con condiciones de contorno asimétricas (plantean distintos valores del coeficiente de transferencia calórica y/o de la temperatura del refrigerante a ambos lados de la placa).

Esquema de Lees: Es implícito, trabaja con tres niveles de tiempo : $(\mathrm{n}-1) \Delta \mathrm{t}$, $n \Delta t$ y $(n+1) \Delta t$ y es totalmente centrado en el punto (i,n). A pesar de ser implícito no es necesario iterar porque todos los coeficientes se calculan al tiempo $\mathrm{n} \Delta \mathrm{t}$.

$$
\begin{aligned}
& \left(\rho C_{p}\right)_{i}^{n} \frac{T_{i}^{n+1}-T_{i}^{n-1}}{2 \Delta t}=\frac{1}{3(\Delta r)^{2}}\left[k_{i+1 / 2}^{n}\left(T_{i+1}^{n+1}-T_{i}^{n+1}+T_{i+1}^{n}-T_{i}^{n}+T_{i+1}^{n-1}-T_{i}^{n-1}\right)-\right. \\
& \left.-k_{i-1 / 2}^{n}\left(T_{i}^{n+1}-T_{i-1}^{n+1}+T_{i}^{n}-T_{i-1}^{n}+T_{i}^{n-1}-T_{i-1}^{n-1}\right)\right]
\end{aligned}
$$

Bonacina \& Comini (1971), Cleland \& Earle (1977, 1979a) y Wilson \& Singh (1987) utilizan este esquema desarrollado inicialmente por Lees (1966). Con este esquema el error de truncación es pequeño. Si bien se pueden utilizar incrementos de tiempo grandes ya que la convergencia del método es incondicional, se ha comprobado que si se utilizan $\Delta t$ demasiado grandes la temperatura puede superar el valor de cambio de fase en un solo paso, ignorando el pico de la curva $C_{p}$ vs. temperatura (este efecto se denomina "peak jumping"), lo que origina tiempos totales menores a los reales al subestimar la carga térmica. Un modo de detectar este error consiste en realizar un balance y comparar el flujo de calor a través de la superficie del alimento con la cantidad total de calor extraída, estos valores difieren cuando se produce el fenómeno de "peak jumping". El pico de la curva $C_{p}$ vs. T se debe a que se utiliza un calor específico aparente que involucra el calor sensible y el calor latente (Figura 2.3). El error mencionado se evita utilizando menores incrementos de tiempo. Los autores han verificado este modelo con datos experimentales de tilosa, un gel de $23 \%$ de metil-hidroxietilcelulosa muy utilizado ya que tiene propiedades termofísicas similares a las de carne vacuna y al congelarse se comporta como la mayoría de los alimentos de alto contenido de agua. 
Pham (1985), para evitar el problema mencionado de "peak jumping", propone calcular el calor específico con un balance de entalpías, luego plantea un esquema de Lees para calcular los perfiles de temperatura.

Esquema de Crank-Nicolson: Es un esquema implícito, la característica es que realiza el balance en el punto de coordenadas (i, $n+1 / 2)$.

$$
\begin{gathered}
\left(\rho C_{p}\right)_{i}^{n} \frac{T_{i}^{n+1}-T_{i}^{n}}{\Delta t}=\frac{1}{2(\Delta r)^{2}}\left[k_{i+1 / 2}^{n}\left(T_{i+1}^{n+1}-T_{i}^{n+1}+T_{i+1}^{n}-T_{i}^{n}\right)-\right. \\
\left.-k_{i-1 / 2}^{n}\left(T_{i}^{n+1}-T_{i-1}^{n+1}+T_{i}^{n}-T_{i-1}^{n}\right)\right]
\end{gathered}
$$

Succar \& Hayakawa (1984) han utilizado este esquema para modelar la congelación de placa plana. Experimentalmente ha sido verificado contra tiempos de congelación de muestras de tilosa.

Esquema entálpico: Es un esquema explícito, la ventaja es que la variación de entalpías es más suave que la variación del producto $\left(P C_{p}\right)$, pero el inconveniente es que paso a paso se debe realizar la conversión de entalpía a temperatura.

$$
\frac{H_{i}^{* n+1}-H_{i}^{* n}}{\Delta t}=\frac{1}{(\Delta r)^{2}}\left[k_{i+1 / 2}^{n}\left(T_{i+1}^{n}-T_{i}^{n}\right)-k_{i-1 / 2}^{n}\left(T_{i}^{n}-T_{i-1}^{n}\right)\right]
$$

Este esquema ha sido utilizado por Joshi \& Tao (1974) quienes estudiaron la congelación de placa plana, utilizando propiedades de carne magra. El método fue verificado con la solución analítica de Neumann, válida para líquidos puros. Sastry (1984) también plantea un esquema usando una entalpía volumétrica como variable dependiente. Mannapperuma \& Singh (1988a) desarrollan un modelo numérico basado en una transformación de entalpías.

Cleland \& Earle (1984a) analizan los resultados obtenidos aplicando los esquemas anteriores al mismo producto y encuentran que todos son igualmente válidos, convergen a idéntico resultado a medida que $\Delta t$ tiende a cero. Para el caso 
particular estudiado los explícitos deben utilizar $\Delta_{\mathrm{t}}$ de $0.5 \mathrm{~s}$ cuando con un esquema implícito se puede trabajar con incrementos de tiempo de $1.5 \mathrm{~s}$ y aún con $\Delta t$ de $6 \mathrm{~s}$ si se usa el esquema de Crank-Nicolson. Actualmente el límite de precisión de los métodos numéricos no puede bajarse de $\pm 10 \%$ debido principalmente a que no se cuenta con valores exactos de las propiedades termofísicas y del coeficiente de transferencia de calor. Es importante destacar que la misma precisión puede obtenerse con un método aproximado.

Para estudiar sistemas con conducción de calor en más de una dirección (cilindro finito, varilla rectangular finita o infinita) Bonacina \& Comini (1973) proponen utilizar el esquema implícito de Lees combinado con el método de direcciones alternantes: se plantean dos esquemas inplícitos, el primero barre la grilla en una dirección y obtiene un perfil de temperaturas ficticias $\mathrm{T}^{*}$ (que no tienen significado físico), estas temperaturas alimentan el segundo esquema del que se obtiene el perfil real $T(x, y, t)$ ó $T(r, z, t)$. Si se tratase de una varilla rectangular finita (prisma rectángulo) se trabaja en tres pasos obteniéndose sucesivamente las temperaturas ficticias $\mathrm{T}^{*}$ y $\mathrm{T}^{* *} \mathrm{y}$, en el tercer paso, la solución $\mathrm{T}(\mathrm{x}, \mathrm{y}, \mathrm{z}, \mathrm{t})$. Los autores citados plantean el modelo numérico para una varilla rectangular infinita, Cleland \& Earle (1979b) estudian la congelación de varillas rectangulares finitas y Cleland \& Cleland (1991) plantean el esquema correspondiente a cilindros finitos.

\subsubsection{SOLUCIONES APROXIMADAS}

Algunas características comunes de los métodos aproximados son:

- Realizan simplificaciones en la ecuación diferencial o regresionan mediante ecuaciones o gráficos resultados obtenidos con métodos "exactos" o determinados experimentalmente.

- Calculan el tiempo total del proceso, y no la evolución de la temperatura con el tiempo.

- Utilizan valores promedio de las propiedades.

- Su aplicación es estrictamente válida para el rango de condiciones operativas en que fueron obtenidos.

- Son fuertemente dependientes de la forma del alimento y, muchos de ellos, han sido desarrollados para un alimento en particular. 
- En general no pueden aplicarse a condiciones operativas variables.

- La gran ventaja que presentan es que el cálculo del tiempo de proceso suele ser sencillo, y puede realizarse aún con una calculadora de mano.

- En general consideran condiciones de contorno simétricas (el mismo valor de temperatura externa y del coeficiente de transferencia calórica en toda la superficie del alimento), el caso particular de congelación asimétrica, que suele encontrarse en la congelación industrial se abordará con mayor extensión en el inciso 2.4.2. Los métodos aproximados conocidos que contemplan asimetría son modificaciones de métodos desarrollados para condiciones simétricas.

El método más antiguo que se conoce es el desarrollado por Plank en 1913, modificado posteriormente por el propio autor en 1941 (Plank, 1963).

Plank realiza distintas suposiciones de modo tal que simplifica el balance diferencial de la ec. (2.1), éstas son:

- La temperatura inicial del producto es uniforme e igual a la de cambio de fase.

- El cambio de fase ocurre a una temperatura constante.

- La diferencia de entalpía $\Delta \mathrm{H}$ sólo involucra calor latente, no hay subenfriamiento del producto posterior al cambio de fase.

- El producto $\rho \mathrm{C}_{\mathrm{p}}$ de la fase congelada tiende a cero con lo que se obtiene un estado pseudoestacionario que origina un perfil lineal de temperaturas.

- Las propiedades térmicas son constantes en cada fase.

- Considera que el flujo de calor en la superficie puede expresarse por la ley de enfriamiento de Newton (condición de contorno de tercer tipo).

La ec. (2.7) es el resultado de integrar el balance de energía con las suposiciones mencionadas:

$$
t_{c}=\frac{\rho_{s} \Delta \hat{H}}{\left(T_{f}-T_{a}\right)}\left(\frac{P D}{h}+\frac{R D^{2}}{k_{s}}\right)
$$

donde tc es el tiempo de congelación, $\rho_{\mathrm{s}} \mathrm{y}$ ks son la densidad y la conductividad térmica del producto congelado, h es el coeficiente de transferencia calórica, $\Delta_{-}$es la diferencia de entalpía entre el estado inicial y final (igual al calor de fusión del 
agua, multiplicado por el contenido inicial de agua del alimento, $\mathrm{Y}_{0}$ ), D es el espesor o el diámetro del alimento, $\mathrm{T}_{\mathrm{f}}$ es la temperatura de comienzo de cambio de fase y $\mathrm{T}_{\mathrm{a}}$ es la temperatura del medio refrigerante.

P y R son constantes que dependen exclusivamente de la geometría, los valores correspondientes se dan en la Tabla 2.1.

Tabla 2.1. Constantes de la ec. (2.7).

\begin{tabular}{||lrr||}
\hline \multicolumn{1}{|c|}{ Geometría } & \multicolumn{1}{c|}{$\mathrm{P}$} & \multicolumn{1}{c|}{$\mathrm{R}$} \\
\hline \hline & & \\
Placa plana infinita & 0.5 & 0.125 \\
Cilindro infinito & 0.25 & 0.0625 \\
Esfera & 0.166 & 0.0416 \\
\hline
\end{tabular}

Todos los supuestos en que se basó Plank no se verifican en el caso más común que se presenta en la congelación industrial ya que :

- La temperatura inicial del alimento generalmente es mayor a $T_{f}$.

- Debido al fenómeno de descenso crioscópico ya mencionado el producto presenta un rango de temperaturas de cambio de fase.

- La temperatura final del producto es mucho menor que la temperatura a la que comienza el cambio de fase, normalmente se fija en $-10^{\circ} \mathrm{C}$ ó $-18^{\circ} \mathrm{C}$.

La ecuación de Plank, a través de los factores de forma $\mathrm{P}$ y R, propone que los tiempos de congelación correspondientes a las tres formas regulares sencillas, bajo las mismas condiciones operativas, mantienen la siguiente relación (que se verifica experimentalmente):

$$
\begin{array}{ccc}
t_{c} \text { (placa plana) } & t_{c} \text { (cilindro infinito) } & : t_{c} \text { (esfera) } \\
6 & : & 3
\end{array}
$$

Los tiempos de congelación calculados por la ec. (2.7) son hasta un $40 \%$ menores que los reales, pero debido a que la ecuación tiene buena base teórica, numerosos autores han planteado diversas modificaciones para desarrollar métodos aproximados con mayor precisión, con algunas de las siguientes características: 
- Introducen factores que corrigen la fórmula original.

- Suman dos términos (o un término y un factor corrector) para tener en cuenta los períodos de preenfriamiento y atemperado.

- Reevalúan las constantes P y R.

Nagaoka y col. (1955) corrigen la ec. (2.7) para considerar una temperatura inicial $\mathrm{T}_{\mathrm{i}}$ distinta de la temperatura de cambio de fase $\mathrm{T}_{\mathrm{f}}$, e incluyen en el valor de

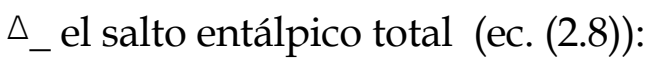

$$
t_{c}=\frac{\left(1+0.008 T_{i}\right) \rho_{s} \Delta \hat{H}}{\left(T_{f}-T_{a}\right)}\left(\frac{P D}{h}+\frac{R D^{2}}{k_{s}}\right)
$$

La fórmula presentada por Levy (1958) es similar a la anterior:

$$
t_{c}=\frac{\left(1+0.008\left(T_{i}-T_{f}\right)\right) \rho_{s} \Delta \hat{H}}{\left(T_{f}-T_{a}\right)}\left(\frac{P D}{h}+\frac{R D^{2}}{k_{s}}\right)
$$

La ec. propuesta por el IIR (1972) considera la diferencia de entalpías entre la temperatura de cambio de fase y la temperatura final $\mathrm{T}_{\mathrm{c}}$ :

$$
t_{c}=\frac{\rho_{s} \Delta \hat{H}}{\left(T_{f}-T_{c}\right)}\left(\frac{P D}{h}+\frac{R D^{2}}{k_{s}}\right)
$$

Se han desarrollado alguna modificaciones más de este tipo, pueden encontrarse en Ramaswamy \& Tung (1984).

Cleland \& Earle $(1977,1979 a)$ realizaron un gran número de experiencias de congelación, cubriendo el rango de condiciones operativas detallado en la Tabla 2.2. En la mayoría de las experiencias utilizaron tilosa, un gel que presenta características y propiedades físicas similares a los alimentos y que posee la ventaja de poder producirse bajo condiciones estandarizadas manteniendo reproducibles sus propiedades.

Regresionaron los datos experimentales de tiempos de congelación de 
muestras de tilosa, obteniendo nuevas expresiones para calcular los factores de forma P y R. El tiempo de congelación se calcula con la ec. (2.10). $\Delta_{-}$es la diferencia de entalpías entre la temperatura de comienzo de cambio de fase Tf y $10^{\circ} \mathrm{C}$, ya que en todas sus experiencias esa es la temperatura final del centro térmico.

Tabla 2.2. Rango de condiciones operativas estudiado experimentalmente por Cleland \& Earle (1977,1979a).

\begin{tabular}{||ccccc||}
\hline \multicolumn{1}{|c}{ Geometría } & $\begin{array}{c}\mathrm{D} \\
(\mathrm{cm})\end{array}$ & $\begin{array}{c}\mathrm{T}_{\mathrm{i}} \\
\left({ }^{\circ} \mathrm{C}\right)\end{array}$ & $\begin{array}{c}\mathrm{T}_{\mathrm{a}} \\
\left({ }^{\circ} \mathrm{C}\right)\end{array}$ & $\begin{array}{c}\mathrm{h} \\
\mathrm{W} /\left(\mathrm{m}^{2 \circ} \mathrm{C}\right)\end{array}$ \\
\hline \hline $\begin{array}{l}\text { Placa Plana } \\
\text { Cilindro infinito } \\
\text { y Esfera }\end{array}$ & 2.5 a 10 & 3 a 35 & $-20 \mathrm{a}-40$ & 10 a 500 \\
\hline
\end{tabular}

Para placa plana infinita:

$$
\begin{gathered}
P=0.5072+0.2018 P k+\operatorname{Ste}\left(0.3224 P k+0.0105 / B^{\prime} i+0.0681\right) \\
R=0.1684+\operatorname{Ste}(0.2740 P k-0.0135)
\end{gathered}
$$

Para cilindro infinito:

$$
\begin{gathered}
P=0.3751+0.0999 P k+\operatorname{Ste}\left(0.4008 P k+0.0710 / B^{\prime} i-0.5865\right) \\
R=0.0133+\operatorname{Ste}(0.0415 P k-0.3957)
\end{gathered}
$$

Para esfera:

$$
\begin{gathered}
P=0.1084+0.0924 P k+\operatorname{Ste}\left(0.231 P k-0.3114 / B^{\prime} i+0.6739\right) \\
R=0.0784+\operatorname{Ste}(0.0386 P k-0.1694)
\end{gathered}
$$

Los números adimensionales que aparecen en las ecuaciones anteriores, números de Stefan, Plank y Biot, se definen como:

$$
\begin{gathered}
\text { Ste }=\frac{\rho_{s} C_{p s}\left(T_{f}-T_{a}\right)}{\Delta H^{*}} \\
P k=\frac{\rho_{0} C_{p 0}\left(T_{i}-T_{f}\right)}{\Delta H^{*}} \\
B^{\prime} i=\frac{h D}{k_{s}}
\end{gathered}
$$

En un trabajo posterior, Cleland \& Earle (1984b) modifican la ec. de Plank para considerar distintas temperaturas finales $T_{c}$. La ecuación (2.14) es válida para 
cualquier geometría (inclusive para formas multidimensionales), la misma se tiene en cuenta en el parámetro EHTD (número de dimensiones equivalentes de transferencia de calor). EHTD vale 1 para placa plana infinita, 2 para cilindro infinito y 3 para esferas, tomando valores intermedios para productos de otras formas.

$$
t_{c}=\frac{\Delta H^{*}}{\operatorname{EHTD}\left(T_{f}-T_{a}\right)}\left(\frac{P D}{h}+\frac{R D^{2}}{k_{s}}\right)\left(1-\frac{1.65 S t e}{k_{s}} \ln \frac{T_{c}-T_{a}}{T_{r e f}-T_{a}}\right)
$$

donde los parámetros $\mathrm{P}$ y $\mathrm{R}$ deben calcularse según:

$$
\begin{gathered}
P=0.5(1.026+0.5808 P k+\operatorname{Ste}(0.2296 P k+0.105)) \\
R=0.125(1.202+\operatorname{Ste}(3.41 P k+0.7336))
\end{gathered}
$$

Se adopta como temperatura de referencia $T_{\text {ref }}=-10^{\circ} \mathrm{C}$.

Los métodos desarrollados por Cleland \& Earle han sido ampliamente utilizados por distintos autores. Si bien el cálculo puede efectuarse con una calculadora de mano, requiere conocer los valores de las propiedades físicas del alimento fresco y congelado y la variación de entalpía del proceso. Se debe recalcar que las regresiones partieron de datos experimentales obtenidos en todos los casos con tilosa, por lo que la extensión del método a otros alimentos deberá hacerse con cierto cuidado.

Mascheroni \& Calvelo (1982) desarrollaron un método analítico, para placa plana, que calcula el tiempo total de congelación, $\mathrm{t}_{\mathrm{c}}$, como la suma de tres tiempos: un tiempo de preenfriamiento $t_{p}$, un tiempo de cambio de fase tce, $y$ un tiempo de atemperado, $\mathrm{tr}$.

$$
t_{c}=t_{p}+t_{c e}+t_{r}
$$

Los autores definen a tp como el tiempo necesario para que la temperatura pase del valor inicial $\mathrm{T}_{\mathrm{i}}$ (uniforme en todo el producto) al valor de comienzo de cambio de fase $\mathrm{T}_{\mathrm{f}}$ en un punto intermedio entre la superficie y el centro térmico. tce es el tiempo necesario para que se produzca la congelación de todo el agua presente a Tf $\mathrm{y}$, por último, tr es el tiempo que transcurre mientras el alimento se enfría desde $\mathrm{T}_{\mathrm{f}}$ hasta la temperatura final $\mathrm{T}_{\mathrm{c}}$ en el centro del producto. 
El tiempo de cambio de fase tce se calcula con la ecuación original de Plank (ec. (2.7)), utilizando una conductividad térmica evaluada a $T_{m}$, valor promedio entre $\mathrm{Tf}_{\mathrm{y}} \mathrm{T}_{\mathrm{a}}$. Los dos tiempos restantes se evalúan con la solución analítica conocida para transferencia de calor sin cambio de fase. Dicha solución se encuentra graficada en forma de curvas de temperatura adimensional vs. tiempo adimensional (Fo), con el número de Biot como parámetro. Para calcular tp se utilizan propiedades físicas promedio entre las evaluadas a $T_{i}$ y a $T_{m}$ y para el cálculo de $t_{r}$ se utilizan las propiedades evaluadas a $\mathrm{T}_{\mathrm{m}}$.

La validez de este modelo fue verificada con valores experimentales obtenidos con muestras de carne vacuna, y con resultados de un modelo numérico. El método permite considerar distintas temperaturas iniciales y finales, y evaluar la contribución de cada período al tiempo total. En general, se verifica que alrededor del 70\% del tiempo total corresponde al período de cambio de fase, y el período de atemperado es levemente superior al de preenfriamiento. Tiene la desventaja de que el cálculo es largo, para cada tiempo intermedio utiliza distintos valores de las propiedades físicas, y se debe disponer de los gráficos o calcular la solución analítica (si bien ésta es exacta su cálculo suele ser complicado).

De Michelis \& Calvelo (1983) extienden esta metodología al cálculo del tiempo de congelación con transferencia de calor en más de una dirección, utilizando el concepto de dimensiones equivalentes, EHTD. Este método tiene una buena base teórica y permite evaluar la contribución de cada etapa al proceso total de congelación, pero análogamente a lo expuesto en el párrafo anterior, el cálculo resulta engorroso ya que se debe recurrir a distintos gráficos y ecuaciones para encontrar el tiempo total.

Hung \& Thompson (1983) modifican la ec. (2.7), para congelación de placas, con transferencia de calor en una única dirección. De acuerdo a su trabajo, $\triangle \mathrm{H}$ debe calcularse según la ec. (2.16) y la diferencia de temperaturas según la ec. (2.17).

$$
\Delta H=C_{p 0}\left(T_{i}-T_{f}\right)+C_{p s}\left(T_{f}-T_{c}\right)+\left(Y_{0}-Y_{n}\right) \lambda_{f}
$$


$\Delta T=\frac{\left(T_{i}-T_{f}\right) C_{p 0}\left(\frac{T_{i}+T_{f}}{2}-T_{a}\right)+\left(T_{f}-T_{c}\right) C_{p s}\left(\frac{T_{f}+T_{c}}{2}-T_{a}\right)+\left(Y_{0}-Y_{n}\right) \lambda_{f}\left(T_{f}-T_{a}\right)}{\Delta H}$

Los autores han considerado que todo el cambio de fase ocurre a una única temperatura, $\mathrm{T}_{\mathrm{f}}$. Las ecuaciones anteriores fueron obtenidas regresionando una serie de medidas experimentales, cubriendo el rango de condiciones de la Tabla 2.3 .

Tabla 2.3. Rango de condiciones operativas estudiado experimentalmente por Hung \& Thompson (1983).

\begin{tabular}{||cccc||}
\hline $\begin{array}{c}\mathrm{D} \\
(\mathrm{m})\end{array}$ & $\begin{array}{c}\mathrm{Ti} \\
\left({ }^{\circ} \mathrm{C}\right)\end{array}$ & $\begin{array}{c}\mathrm{Ta}_{\mathrm{a}} \\
\left({ }^{\circ} \mathrm{C}\right)\end{array}$ & $\begin{array}{c}\mathrm{h} \\
\mathrm{W} /\left(\mathrm{m}^{2 \circ} \mathrm{C}\right)\end{array}$ \\
\hline \hline & & & \\
\hline
\end{tabular}

Las ecuaciones para recalcular P y $\mathrm{R}$ fueron halladas con un método de regresión lineal múltiple:

$$
\begin{gathered}
P=0.7306-1.083 P k+\operatorname{Ste}\left(15.4 U-15.43+0.01329 S_{t e} / B^{\prime} i\right) \\
R=0.2079-0.2656 U \text { Ste }
\end{gathered}
$$

En las ecuaciones anteriores $\mathrm{Ste}, \mathrm{Pk}$ y $\mathrm{Bi}$ ' son números adimensionales definidos en las ecs. (2.11), (2.12) y (2.13) respectivamente y $\mathrm{U}$ es una temperatura adimensional.

Este modelo fue testeado con valores experimentales de congelación de carne magra, carne picada, carpa y puré de papa. La mayor dificultad para utilizarlo reside en la necesidad de conocer el parámetro $\mathrm{Y}_{\mathrm{n}}$, fracción de agua que queda sin congelar.

Bazán \& Mascheroni (1984) estudian la congelación de carnes ovinas, determinan experimentalmente las propiedades termofísicas y resuelven el balance de energía por diferencias finitas para geometría de placa plana. Regresionando los resultados numéricos obtienen una ecuación sencilla para el cálculo del tiempo de congelación; que difiere según la temperatura final sea $-10^{\circ} \mathrm{C}$ (ec.

Cap. 2-20 
(2.18)) ó $-18^{\circ} \mathrm{C}$ (ec. (2.19)):

$$
\begin{aligned}
t_{c} & =77.979 \frac{L^{2}}{\alpha_{0}}\left(B i^{-1}+0.19387\right)\left(1+9.001110^{-3}\left(T_{i}-T_{f}\right)\right)\left(T_{f}-T_{a}\right)^{-1.01353} \\
t_{c} & =135.564 \frac{L^{2}}{\alpha_{0}}\left(B i^{-1}+0.18327\right)\left(1+7.991910^{-3}\left(T_{i}-T_{f}\right)\right)\left(T_{f}-T_{a}\right)^{-1.13292}
\end{aligned}
$$

Rubiolo de Reinick \& Schwartzberg (1986) desarrollan un método rápido válido tanto para congelación como para descongelación, que puede ser usado en alimentos rectangulares, con dos o cuatro caras expuestas a la transferencia de calor, las condiciones externas pueden ser simétricas o asimétricas. El método se restringe a bajos números de Biot $\left(\mathrm{Bi}^{\prime} \leq 3\right)$. El punto de partida del desarrollo es la solución analítica para el problema sin cambio de fase y $\mathrm{Bi}=0$. La gran ventaja frente a los métodos ya analizados es que además de calcular el tiempo total de proceso (ec. (2.20)) presenta un modo simple de calcular una temperatura media entálpica $T_{m e}$ en función del tiempo, a medida que transcurre el cambio de fase. Esta se define como la temperatura a la que la función $\mathrm{H}(\mathrm{T})$ iguala a la entalpía total del alimento.

$$
t_{c}=\frac{W E(T)}{\sum h_{j} S_{j} F\left(B i_{j}^{\prime}\right)}
$$

donde el factor $\mathrm{E}(\mathrm{T})$ surge de integrar el balance usando un calor específico efectivo (Schwartzberg, 1976) que contempla el calor de cambio de fase, depende de los valores de las temperaturas y de las propiedades termofísicas; y $F\left(B_{i}{ }^{\prime}\right)$ es un factor de corrección, que es función del número de Biot de cada cara (el subíndice j se refiere a la cara de superficie $\mathrm{S}_{\mathrm{j}}$ ).

$$
\begin{gathered}
E(T)=C_{p s} \ln \left[\frac{G\left(T_{i}-T_{a}\right)}{T_{m e}-T_{a}}\right]+\frac{D_{l}}{\left(T_{w}-T_{a}\right)^{2}}\left[\ln \left(G D_{2}\right)+D_{3}\right] \\
G=G_{i} G_{j} G_{k} \text { donde cada } G_{l}=1-0.01 B^{\prime} i_{l} \text { siendo } l=i, j, k \\
D_{l}=Y_{0}-b N_{s}\left(T_{w}-T_{i}\right) \lambda_{f} \\
D_{2}=\frac{\left(T_{w}-T_{m e}\right)\left(T_{a}-T_{i}\right)}{\left(T_{w}-T_{i}\right)\left(T_{a}-T_{m e}\right)} \text { y } \quad D_{3}=\frac{\left(T_{i}-T_{m e}\right)\left(T_{w}-T_{a}\right)}{\left(T_{w}-T_{a}\right)\left(T_{w}-T_{m e}\right)}
\end{gathered}
$$

Cap. $2-21$ 


$$
F\left(B^{\prime} i\right)=\frac{1}{1+0.17 B^{\prime} i} \quad F\left(B^{\prime} i\right)=\frac{1}{1+\frac{B^{\prime} i}{6}+\frac{B^{\prime} i^{2}}{224}}
$$

Pham (1984) presenta un método en el que introduce ciertas aproximaciones analíticas para modificar la ecuación original de Plank. Basándose en el método de Mascheroni y Calvelo, divide el proceso en tres etapas: preenfriamiento, cambio de fase y subenfriamiento (ec. (2.15)).

Para calcular los tiempos tp (ec. (2.23)) y tr (ec. (2.24)), que corresponden a los períodos sin cambio de fase, corrige la "ley de enfriamiento" de Newton, teniendo en cuenta la resistencia interna a la transferencia de calor.

$$
t_{p}=t_{l}=\frac{\Delta H_{1}}{h A \Delta T_{m l}}\left(1+\frac{B i_{1}}{6}\right)
$$

donde:

$$
\begin{gathered}
\Delta H_{1}=\rho_{0} C_{p 0}\left(T_{i}-T_{f m}\right) V \\
\Delta T_{m l}=\frac{\left(T_{i}-T_{a}\right)-\left(T_{f m}-T_{a}\right)}{\ln \frac{T_{i}-T_{a}}{T_{f m}-T_{a}}} \\
\mathrm{y} \quad t_{r}=t_{3}=\frac{\Delta H_{3}}{h A \Delta T_{m 3}}\left(1+\frac{B^{\prime} i}{6}\right)
\end{gathered}
$$

donde:

$$
\begin{gathered}
\Delta_{H_{3}}=\rho_{s} C_{p s}\left(T_{f m}-T_{0}\right) V \\
\Delta T_{m 3}=\frac{\left(T_{f m}-T_{a}\right)-\left(T_{0}-T_{a}\right)}{\ln \frac{T_{f m}-T_{a}}{T_{0}-T_{a}}}
\end{gathered}
$$

To es una temperatura media final, que se calcula conociendo la temperatura final del centro térmico $\mathrm{T}_{\mathrm{c}} \mathrm{y}$ el número de Biot.

$$
T_{0}=T_{c}-\left(T_{c}-T_{a}\right)\left(2+\frac{4}{B^{\prime} i}\right)
$$

El cálculo del tiempo de cambio de fase, tce, lo realiza con la ecuación de Plank (ec. (2.26)), considerando una temperatura media de cambio de fase Tfm que es aproximadamente $1.5^{\circ} \mathrm{C}$ menor que la temperatura de comienzo de cambio de fase $T_{f}$. 


$$
t_{c e}=t_{2}=\frac{\Delta H_{2}}{h A\left(T_{f m}-T_{a}\right)}\left(1+\frac{B^{\prime} i}{4}\right)
$$

donde:

$$
\Delta H_{2}=V \lambda_{f, e f}^{*}
$$

Bi1 es el número de Biot promedio de los evaluados con las propiedades del fresco y del congelado respectivamente y con el espesor total como longitud característica.

Del análisis de los resultados surge que esta modificación es buena cuando la fracción de calor sensible no es muy pequeña respecto a la fracción de calor latente y cuando los números de Biot son bajos, lo que se cumple con temperaturas inicial y final distantes del punto de congelación.

En un trabajo posterior Pham (1986) corrige el método considerando que todo el cambio de fase ocurre a una temperatura media Tfm común a todos los alimentos, obtenida al regresionar todos los valores experimentales de tc publicados. Dicha temperatura de cambio de fase queda definida por la siguiente ecuación:

$$
T_{\text {fin }}=1.8+0.263 T_{c}+0.105 T_{a}
$$

Reagrupa las etapas, sólo tiene una de preenfriamiento, y otra de cambio de fase más el subenfriamiento. En ambas considera $\left(1+\mathrm{Bi}^{\prime} / 4\right)$ en el factor que corrige la ley de enfriamiento $\left(\mathrm{Bi}^{\prime}=\mathrm{hD} / \mathrm{ks}\right)$.

El tiempo de congelación puede calcularse con la ec. (2.28):

$$
\begin{gathered}
t_{c}=\frac{V}{h A}\left(\frac{\Delta H_{1}^{*}}{\Delta T_{1}}+\frac{\Delta H_{2}^{*}}{\Delta T_{2}}\right)\left(1+\frac{B^{\prime} i}{4}\right) \\
\Delta H_{1}^{*}=\rho_{0} C_{p 0}\left(T_{i}-T_{f m}\right) \\
\Delta H_{2}^{*}=\lambda_{f, e f}^{*}+\rho_{s} C_{p s}\left(T_{f m}-T_{c}\right) \\
\Delta T_{1}=\frac{T_{i}+T_{f m}}{2}-T_{a} \\
\Delta T_{2}=T_{f m}-T_{a}
\end{gathered}
$$

Este método tiene un error de predicción: calcula un tiempo de congelación finito en el caso de que la temperatura final en el centro térmico coincide con la 
temperatura del medio refrigerante, $T a$.

Hasta aquí se han descripto los métodos de predicción más conocidos y utilizados, la lista de métodos existentes es bastante extensa, pero la mayoría de ellos presentan restricciones ya sea en cuanto a la geometría, a las condiciones de trabajo o respecto del producto.

\subsection{METODOS DE PREDICCION DESARROLLADOS}

Luego de realizar una exhaustiva revisión de los métodos aproximados conocidos, podemos concluir que no existe un método generalizado de buena precisión que calcule tc con una única ecuación, contemplando un rango amplio de las variables independientes: tamaño, propiedades físicas, condiciones de trabajo. Todos los existentes necesitan del cálculo de coeficientes variables, utilizan más de una valor de cada propiedad termofísica, interpolan datos de tablas o gráficos, etc.

Por ello se consideró importante desarrollar un método de predicción que cumpliera con los siguientes requisitos:

- Debe ser simple y preciso.

- Debe usar propiedades físicas a una única temperatura (mayor que $0^{\circ} \mathrm{C}$ ).

- Debe ser válido para cualquier temperatura final (inferior a $-5^{\circ} \mathrm{C}$ ).

- Debe ser válido para cualquier alimento (de estructura homogénea y de alto contenido de agua).

Para llegar a la forma final del método propuesto (ec. (2.55)), se fueron cumpliendo varias etapas.

Inicialmente se obtuvieron los perfiles de temperatura en función del tiempo en un alimento que se congela, resolviendo el balance diferencial de energía térmica por un método numérico. En la ec. (2.29) se presenta el balance general de Energía partiendo de las ecuaciones básicas de fenómenos de transporte: 


$$
\rho \frac{D \hat{H}}{D t}=-(\Delta \cdot \vec{q})-(\tau: \Delta \vec{v})+\frac{D p}{D t}
$$

El miembro de la izquierda representa la variación de entalpía con el tiempo ( $\hat{\mathrm{H}}$ : entalpía por unidad de masa). La derivada sustancial puede reemplazarse por la derivada parcial, al considerar despreciable los efectos convectivos por tratarse de un alimento sólido (o al menos de geles).

En el segundo miembro, el primer término representa el flujo de calor que se transfiere por conducción; q puede vincularse con la temperatura por medio de la ley de Fourier:

$$
\vec{q}=k \Delta T
$$

El término $(\tau: \nabla v)$ donde $\tau$ es el tensor esfuerzo viscoso y $\nabla v$ el gradiente de velocidades es nulo ya que no hay disipación viscosa al tratarse de un alimento sólido y el tercer término (derivada de la presión respecto del tiempo) también es nulo ya que la congelación, en equipos industriales, transcurre a presión constante. Al realizar el balance de energía se supone que durante la congelación no se producen reacciones químicas, lo cual no es estrictamente cierto ya que ocurren reacciones enzimáticas y se insolubilizan proteínas, pero se admite que el calor involucrado en estas reacciones es despreciable frente al calor de cambio de fase. Se supone además que no se produce recristalización durante la congelación, en caso contrario deberíamos contemplar la energía superficial puesta en juego en la desaparición de los cristales pequeños y el crecimiento de los cristales más grandes.

La ec. (2.31) nos da el balance simplificado:

$$
\rho \frac{\partial \hat{H}}{\partial t}=-\Delta(k \Delta T)
$$

Para resolver esta ecuación debemos contar con una relación que vincule la entalpía con la presión, la temperatura y la composición. 


$$
d \hat{H}=\left(\frac{\partial \hat{H}}{\partial T}\right)_{p, w_{i}} d T+\left(\frac{\partial \hat{H}}{\partial p}\right)_{T, w_{i}} d p+\sum_{I}^{N}\left(\frac{\partial \hat{H}}{\partial w_{i}}\right)_{T, p, w_{j} \neq i} d w_{i}
$$

donde es:

$$
\left(\frac{\partial \hat{H}}{\partial T}\right)_{p, w_{i}}=C_{p} \quad y\left(\frac{\partial \hat{H}}{\partial p}\right)_{T, w_{i}}=0
$$

En la resolución del balance se utilizaron propiedades de carne vacuna, admitiendo que el tejido cárneo parcialmente congelado se puede modelar como constituido por tres componentes: agua, hielo y tejido seco (un análisis similar puede hacerse para tejidos vegetales). Como la sumatoria de las fracciones másicas de todos los componentes es igual a 1, sólo tenemos dos fracciones másicas independientes, por ejemplo wa (fracción másica de agua líquida) y wt (de tejido). En la congelación el agua se transforma en hielo, y la fracción de tejido seco permanece invariable $\left(\mathrm{dw}_{\mathrm{t}}=0\right)$.

$$
\sum_{l}^{2}\left(\frac{\partial \hat{H}}{\partial w_{i}}\right)_{p, T, w_{j \neq i}}=\left(\frac{\partial \hat{H}}{\partial w_{a}}\right)_{p, T, w_{t}} d w_{a}
$$

donde:

$$
\left(\frac{\partial \hat{H}}{\partial w_{a}}\right)_{p, T, w_{t}}=\lambda_{f}
$$

En la ecuación anterior se ha considerado que el calor de disolución de las sales en el agua es constante, no es función del contenido de hielo.

La fracción másica de agua puede expresarse en función del contenido inicial de humedad del alimento Yo y de la fracción másica de hielo, w:

$$
w_{a}=Y_{0}(1-w)_{-} \frac{d w_{a}}{d T}={ }_{-} Y_{0} \frac{d w}{d T}
$$

Reemplazando las ecuaciones anteriores la ec. (2.32) puede reescribirse Cap. 2-26 
como:

$$
d \hat{H}=C_{p} d T+\lambda_{f} d w_{a}=\left(C_{p}-\lambda_{f} Y_{0} \frac{d w}{d T}\right) d T
$$

Considerando que la fracción de hielo w es función del contenido inicial de agua, Yo, y de la temperatura T (ec. (2.38), Mascheroni \& Calvelo (1978)) y definiendo, según la ec. (2.39), un calor específico aparente que considere el calor sensible y el calor latente, se simplifica aún más la expresión del balance diferencial de energía térmica (ec. 2.37):

$$
\begin{gathered}
w=1.1866-\frac{0.1866}{Y_{0}}+\frac{2.7013}{T}\left(\frac{1-Y_{0}}{Y_{0}}\right) \\
C_{p, a p}=C_{p}-\lambda_{f} Y_{0} \frac{d w}{d T} \\
\rho_{C_{p, a p}} \frac{\partial T}{\partial t}=\Delta(k \Delta T)=\frac{\partial}{\partial r}\left(k \frac{\partial T}{\partial r}\right)+\frac{a^{\prime} k}{r} \frac{\partial T}{\partial r}
\end{gathered}
$$

Nota: En adelante llamaremos $C_{p}$ al calor específico aparente.

En la ec. (2.40) se ha desarrollado el gradiente de temperatura, utilizando $\mathrm{r}$ como coordenada genérica de posición (recordemos que sólo nos interesa estudiar la transferencia de calor en una única dirección). El parámetro a' vale 0 para placa plana infinita, 1 para cilindro infinito y 2 para esfera.

Las propiedades utilizadas con el modelo matemático son las siguientes (Sanz y col., 1989):

$$
\begin{aligned}
& T_{f}=\frac{1-Y_{0}}{0.06908-0.4393 Y_{0}} \\
& \rho=1053 . \quad T \geq T_{f} \\
& \rho=\frac{1053 .}{0.98221+0.1131 Y_{0}+0.25746\left(1-Y_{0}\right) T^{-1}} T<T_{f} \\
& C_{p}=1448\left(1-Y_{0}\right)+4187 Y_{0} \quad T \geq T_{f} \\
& C_{p}=3874-2534 Y_{0}+902893\left(1-Y_{0}\right) T^{-2} \quad T<T_{f}
\end{aligned}
$$


El modelo utilizado para describir la conductividad térmica de carne parcialmente congelada implica suponer un arreglo particular de hielo y fibras parcialmente deshidratadas. Se admite que el agua es un medio disperso en una matriz sólida formada por el resto de los constituyentes del tejido. Cuando la temperatura es superior a $\mathrm{T}_{\mathrm{f}}$, el agua se encuentra totalmente dentro de las fibras (Mascheroni $\mathrm{y}$ col., 1977). A medida que la temperatura desciende y se produce el cambio de fase, aparece hielo extracelular que crece a expensas del agua dispersa en el tejido.

Al modelar la conductividad térmica debe diferenciarse, en geometría plana, la transferencia de calor perpendicular a las fibras de la situación paralela a las mismas.

En el primer caso la conducción se efectúa a través de dos caminos: hielo continuo y tejido con hielo en serie. Este modelo conduce a las siguientes ecuaciones (Sanz y col., 1989):

$$
\begin{gathered}
k=0.0866+0.501 Y_{0}+5.05210^{-4} Y_{0} T \quad T \geq T_{f} \\
k=0.378+1.376 Y_{0}+0.930 T^{-1} \quad T<T_{f}
\end{gathered}
$$

En el caso paralelo existe transferencia calórica en paralelo entre tres disposiciones: hielo, fibra e hielo-fibra en serie. Las ecuaciones que se obtienen son las siguientes (Sanz y col., 1989):

$$
\begin{gathered}
k=0.1075+0.501 Y_{0}+5.05210^{-4} Y_{0} T \quad T \geq_{T_{f}} \\
k=0.398+1.448 Y_{0}+0.985 T^{-1} \quad T<T_{f}
\end{gathered}
$$

$\mathrm{Si}$ bien este modelo numérico se verificó experimentalmente con propiedades de carne, es válido para cualquier otro sistema donde se cumplan las siguientes condiciones: la presión se mantiene constante, no hay aportes convectivos ni disipación viscosa y es correcta la suposición de tres componentes. 


\subsubsection{CONGELACION SIMETRICA.}

Al establecer las condiciones de contorno en geometría plana pueden diferenciarse dos situaciones: condiciones simétricas y asimétricas. En la mayoría de los casos se admite que la congelación se produce bajo condiciones de contorno simétricas; que en geometría plana significa que tanto el coeficiente de transferencia calórica como la temperatura externa tienen idéntico valor en ambas caras del alimento. En cilindros y esferas, si admitimos que tanto $h$ como $T_{a}$ son uniformes en toda la superficie del alimento la congelación será simétrica.

Las condiciones inicial y de contorno que se aplican a la ec. (2.40) al simular la operación en congeladores industriales son las siguientes:

$$
\begin{array}{cc}
T=T_{i} & 0 \leq r \leq L \quad t \leq 0 \\
\frac{\partial T}{\partial r}=0 & r=0 \quad t>0 \\
-k \frac{\partial T}{\partial r}=h\left(T-T_{a}\right) \quad r=L \quad t>0
\end{array}
$$

La ecuación diferencial fue resuelta con un esquema de diferencias finitas explícitas, utilizando diferencias adelantadas para el tiempo (con error de truncación del orden de $\Delta t$ ) y diferencias centradas para las derivadas respecto del espacio (con error de truncación del orden de $\left.(\Delta \mathrm{r})^{2}\right)$ :

$$
\begin{gathered}
\frac{\partial T}{\partial t}=\frac{T_{i}^{n+1}-T_{i}^{n}}{\Delta t} \\
\frac{\partial T}{\partial r}=\frac{T_{i+1}^{n}-T_{i-1}^{n}}{2 \Delta r} \\
\frac{\partial^{2} T}{\partial r^{2}}=\frac{T_{i+1}^{n}-2 T_{i}^{n}+T_{i-1}^{n}}{\Delta r^{2}}
\end{gathered}
$$

Cap. 2-29 
Se usó una malla equiespaciada colocando el origen de coordenadas en el centro. En ese punto $\mathrm{i}=1(\mathrm{r}=0)$ y en el borde $\mathrm{i}=\mathrm{I}(\mathrm{r}=\mathrm{L})$. Las propiedades se evaluaron en los puntos de la grilla, en el tiempo anterior $\mathrm{n}(\Delta \mathrm{t})$.

La ecuación discretizada, reordenada, para placa plana infinita, es:

$$
T_{i}^{n+1}=T_{i}^{n}+\frac{\Delta t k_{i}^{n}}{(\Delta r)^{2} \rho_{i}^{n} C_{p i}^{n}}\left(T_{i+1}^{n}-2 T_{i}^{n}+T_{i-1}^{n}\right)+\frac{\Delta t}{4(\Delta r)^{2} \rho_{i}^{n} C_{p i}^{n}}\left(k_{i+1}^{n}-k_{i-1}^{n}\right)\left(T_{i+1}^{n}-T_{i-1}^{n}\right)
$$

La ecuación anterior es válida para los puntos interiores, $2 \leq \mathrm{i} \leq \mathrm{I}-1$. Para los extremos, $\mathrm{i}=1$ e $\mathrm{i}=\mathrm{I}$ surgen expresiones particulares al aplicar las condiciones de contorno.

Para $\mathrm{i}=1$ :

$$
T_{I}^{n+1}=T_{1}^{n}+\frac{4 \Delta t k_{l}^{n}}{(\Delta r)^{2} \rho_{1}^{n} C_{p l}^{n}}\left(T_{2}^{n}-T_{I}^{n}\right)
$$

y para $\mathrm{i}=\mathrm{I}$ :

$$
T_{I}^{n+1}=T_{I}^{n}+\frac{2 \Delta t}{(\Delta r)^{2} \rho_{I}^{n} C_{p I}^{n}}\left[h \Delta r\left(T_{a}-T_{I}^{n}\right)-k_{I-I / 2}\left(T_{I}^{n}-T_{I-1}^{n}\right)\right]
$$

Para que el esquema converja, el coeficiente que multiplica a la temperatura del tiempo anterior $T_{i}^{n} 8$ en la ec. (2.47) debe ser positivo, por lo tanto el incremento de tiempo $\Delta \mathrm{t}$ no es arbitrario sino que queda determinado por el incremento de espacio y los valores de las propiedades físicas, debiendo cumplirse la condición expresada por la ec. (2.50):

$$
\Delta t \leq \frac{(\Delta r)^{2} \rho C_{p}}{2 k}
$$

Para cilindros y esferas el término $\frac{a^{\prime} k}{r} \frac{\partial T}{\partial r}$ del balance (ec.(2.40)) queda 
indeterminado en el centro del alimento, en ese punto se aplica la regla de L'Hôpital y se aproxima por la derivada segunda de la temperatura respecto de la posición.

Este modelo numérico ha sido extensamente empleado y correctamente testeado con valores experimentales de congelación y descongelación (Mascheroni \& Calvelo, 1980; Mascheroni, 1982; Bazán \& Mascheroni, 1984); ha sido utilizado en este trabajo ya que el objetivo perseguido era desarrollar un método aproximado de predicción y no implementar un nuevo modelo numérico.

El programa de cálculo fue implementado en FORTRAN 77, para placa plana se usó una grilla de 37 puntos y un paso de tiempo de $1 \mathrm{~s}$; para cilindro infinito una grilla de 41 puntos y $\Delta \mathrm{t}$ de $0.75 \mathrm{~s}$ y, finalmente, para esfera, se usó una grilla de 41 puntos y $\Delta t=0.4 \mathrm{~s}$. Numéricamente se obtuvieron las historias térmicas de distintos puntos. El análisis se centró en el punto que, en todo momento se encuentra a mayor temperatura. Dicho punto, definido como el centro térmico, coincide con el centro geométrico en las tres formas estudiadas.

Con el modelo numérico se estudió un rango muy amplio de condiciones operativas, de modo de cubrir todos los valores que es posible hallar en la práctica industrial, rango que se detalla en la Tabla 2.4.

Tabla 2.4. Rango de variación de condiciones operativas estudiado con el modelo numérico.

\begin{tabular}{||cccc||}
\hline Geometría & $\begin{array}{c}\mathrm{Ti} \\
\left({ }^{\circ} \mathrm{C}\right)\end{array}$ & $\begin{array}{c}\mathrm{Ta} \\
\left({ }^{\circ} \mathrm{C}\right)\end{array}$ & $\mathrm{Bi}$ \\
\hline \hline Placa plana $(\perp)$ & 2 a 25 & -25 a -45 & 1 a 50 \\
Placa plana $(\|)$ & 2 a 20 & -25 a -45 & 1 a 60 \\
Cilindro infinito & 7 a 25 & -20 a -40 & 1 a 30 \\
Esfera & 2 a 25 & -25 a -45 & 1 a 20 \\
\hline
\end{tabular}

La Figura 2.6 nos muestra una serie de curvas de congelación, de placa plana, con transferencia de calor perpendicular a las fibras. Se ha graficado la evolución de la temperatura del centro térmico en función del tiempo, para 
distintas combinaciones de los parámetros $\mathrm{T}_{\mathrm{i}}, \mathrm{T}_{\mathrm{a}}$ y Bi. En todos los casos el semiespesor del producto es de $3.6 \mathrm{~cm}$.

Puede observarse que todas las curvas tienen la misma forma, si bien parten de distintos valores iniciales, en todas se observa una zona plana, donde se produce la mayor parte del cambio de fase, y luego la región final de atemperado. Para formular un método aproximado de predicción del tiempo de congelación tendríamos que encontrar una expresión que vincule la temperatura del centro térmico $T_{c}$ con el tiempo. Dicha expresión no necesariamente deberá valer para toda la curva de congelación, sino que debe cumplirse en el tramo final (para calcular el tiempo de congelación la temperatura $T_{c}$ deberá tomar valores inferiores a $-5^{\circ} \mathrm{C}$, ya que a esa temperatura podemos admitir que se ha producido casi totalmente el cambio de fase).

Empíricamente, se buscó una variable adimensional $X$ que reemplazara al tiempo $t$ de modo que la gráfica de $T_{c}$ vs. $X$ resultase en una única curva, considerando aún los casos extremos de los parámetros indicados en la Tabla 2.4. Toda la complejidad del problema se vuelca en la definición de la variable $X$ que deberá contemplar todos los parámetros del proceso además del tiempo t: tamaño del producto $\mathrm{L}$ (semiespesor en geometría plana o radio para cilindros y esferas) y propiedades físicas del alimento ( $\rho, C_{p}$ y k), temperatura del refrigerante $T_{a}$, temperatura inicial $T_{i}$, coeficiente de transferencia calórica $h$.

Después de probar distintas formas, basadas en resultados de los modelos teóricos aproximados, la variable $\mathrm{X}$ quedó definida por la siguiente ecuación:

$$
X=\frac{F o\left(\frac{T_{a}-T_{f}}{T_{f}}\right)^{m}}{\left(\frac{1}{B i}+c\right)\left(\frac{T_{f}-T_{i}}{T_{f}}\right)^{n}}
$$




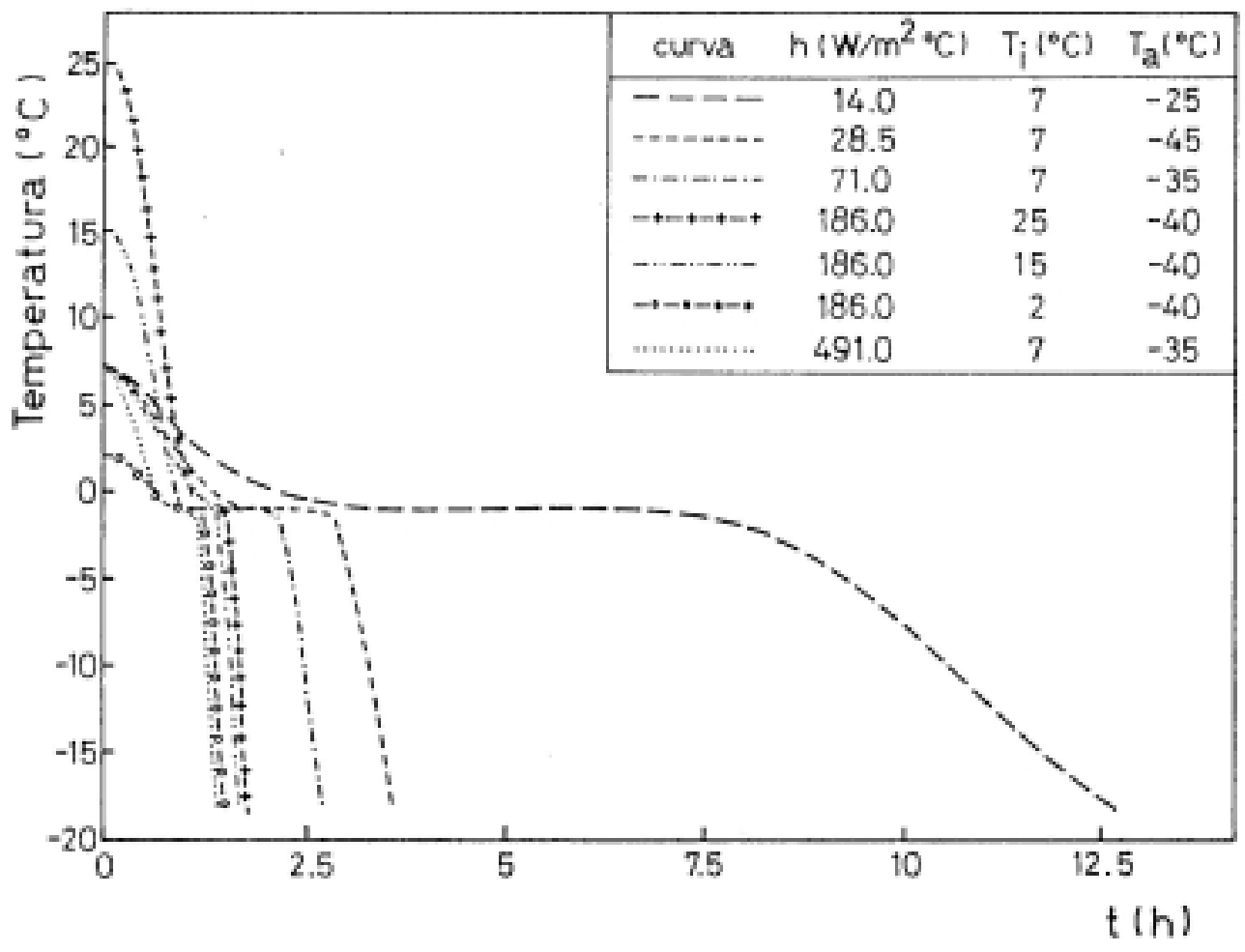

Figura 2.6. Curvas de congelación, placa plana, correspondientes a distintas condiciones operativas.

Las propiedades físicas que aparecen en el número de Fourier (difusividad térmica $\alpha$ ) y en el número de Biot (conductividad térmica k) corresponden al estado fresco (sin congelar) ya que son más fáciles de obtener de la bibliografía, calcular o determinar experimentalmente.

Las constantes $\mathrm{c}, \mathrm{m}$ y $\mathrm{n}$ son constantes empíricas que dependen exclusivamente de la geometría, se presentan en la Tabla 2.5 . 
Tabla 2.5. Constantes empíricas de la ec. (2.51).

\begin{tabular}{||lccc||}
\hline \multicolumn{1}{|c}{ Geometría } & $\mathrm{c}$ & $\mathrm{m}$ & $\mathrm{n}$ \\
\hline \hline Placa plana $(\perp)$ & 0.18 & 1.04 & 0.09 \\
Placa plana $(\|)$ & 0.16 & 1.04 & 0.10 \\
Cilindro infinito & 0.17 & 1.00 & 0.09 \\
Esfera & 0.18 & 0.90 & 0.06 \\
\hline \hline
\end{tabular}

En la Figura 2.7 se han graficado las curvas de congelación ya mostradas en la Figura 2.6, pero en función de X.

En la Figura 2.8 se ha graficado la temperatura del centro, en la zona final de la curva de congelación, en función de $X$, para las cuatro geometrías estudiadas. En todos los casos las líneas punteadas corresponden a los valores extremos de $\mathrm{X}$ para cada temperatura, $\mathrm{v}$ la línea llena al valor promedio de $\mathrm{X}$.

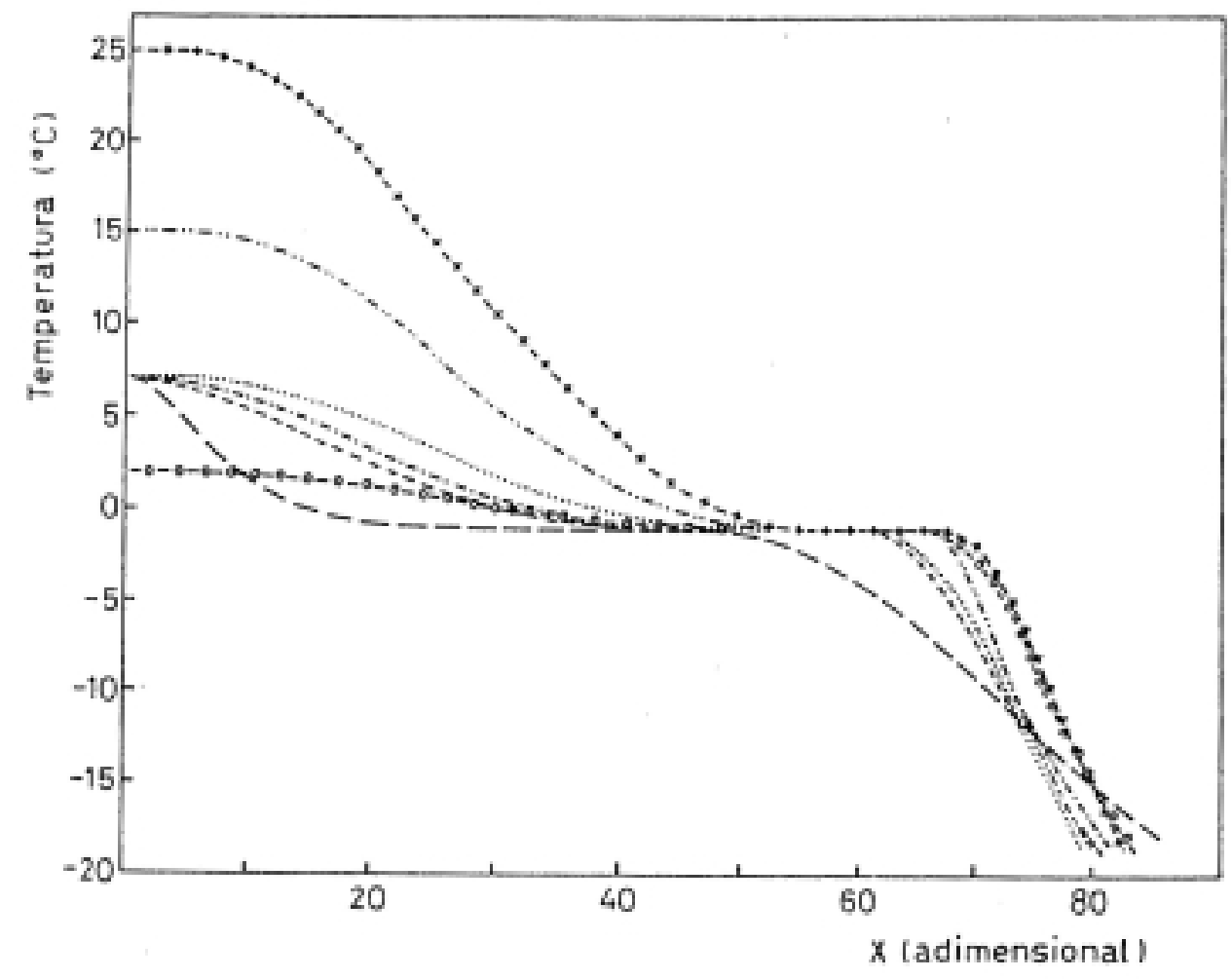

Figura 2.7. Temperatura del centro térmico vs. X, placa plana infinita, para distintas condiciones operativas. 


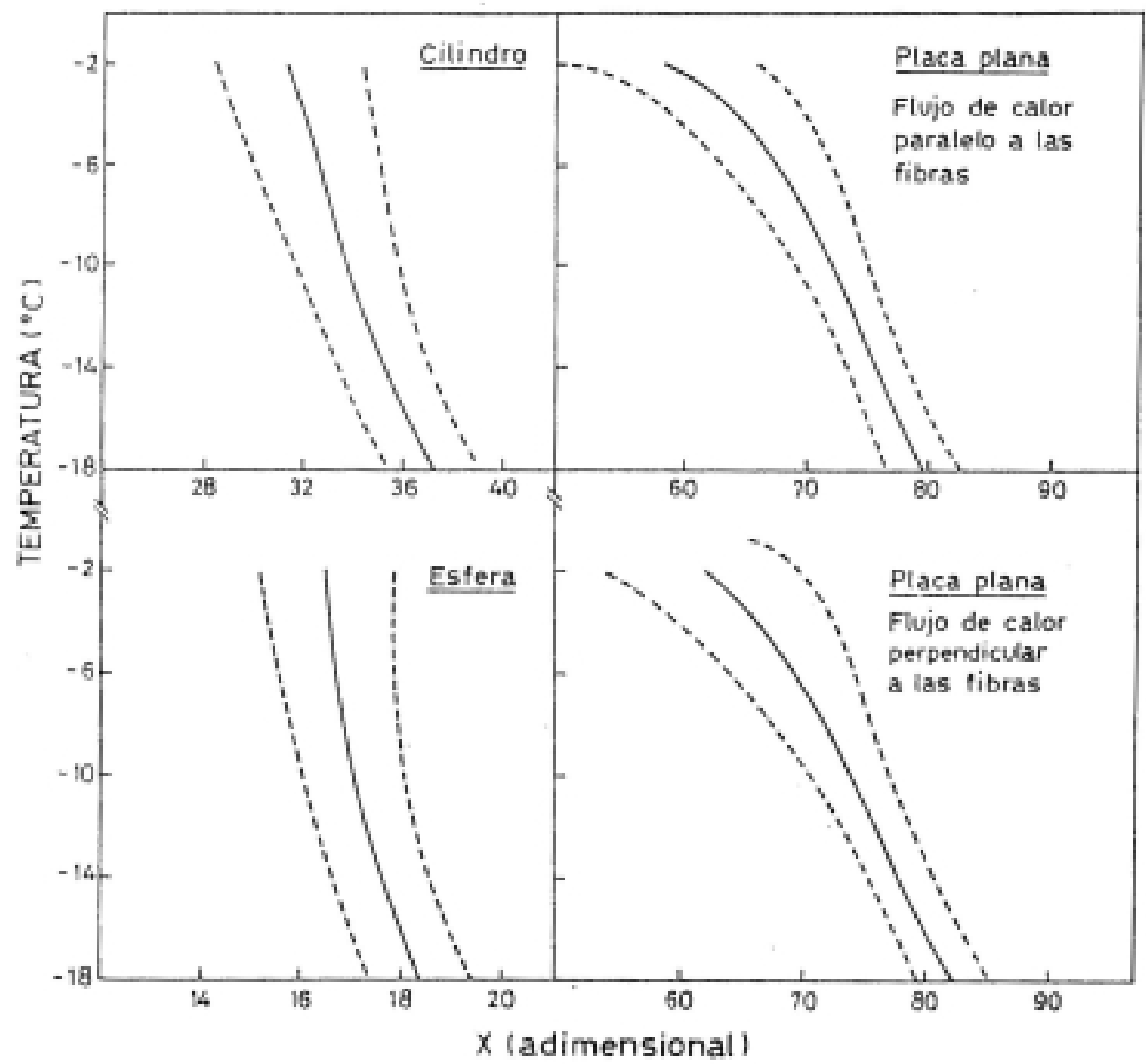

Figura 2.8. Temperatura del centro térmico vs. X, para las cuatro geometrías estudiadas.

En la ec. (2.51) aparece como variable la temperatura de comienzo de cambio de fase $\mathrm{T}_{\mathrm{f}}$. Recordemos que el método fue desarrollado utilizando resultados numéricos obtenidos con propiedades de carne vacuna, para la cual $\mathrm{T}_{\mathrm{f}}$ es aproximadamente $-1^{\circ} \mathrm{C}$ (varía un poco dependiendo del contenido inicial de agua $\mathrm{Y}_{0}$ ). Al utilizar este método con otros alimentos se comprobó que ajusta mejor la expresión siguiente, donde se ha reemplazado Tf por $-1^{\circ} \mathrm{C}$ (en esta expresión debe trabajarse necesariamente con el valor de temperatura en grados Centígrados): 


$$
X=\frac{F o\left(-T_{a}-1\right)^{m}}{\left(\frac{1}{B i}+c\right)\left(1+T_{i}\right)^{n}}
$$

El primer método de predicción propuesto se basaba en tomar de la Figura 2.8 el valor de X para una temperatura final determinada (de la línea llena promedio correspondiente, según la geometría). Luego, reordenando la ec. (2.52) y desadimensionalizando el tiempo (del número de Fourier, Fo) se calculaba el tiempo de congelación tc:

$$
t_{c}=X \frac{L^{2}}{\alpha_{0}}\left(\frac{1}{B i}+c\right)\left(1+T_{i}\right)^{n}\left(-T_{a}-1\right)^{-m}
$$

La desventaja que presenta esta metodología de cálculo reside en que es necesario tomar el valor de $\mathrm{X}$ del gráfico, agregando un nuevo error (de lectura) en la predicción de tc.

Tomando como base la ec. (2.52) que define la variable $X$, y admitiendo que la relación entre la temperatura del centro térmico, Tc y $\mathrm{X}$ puede expresarse por una recta, tal como lo muestran las Figuras 2.7 y 2.8 (ec. (2.54)), se plantea una nueva ecuación de predicción, ec. (2.55), debiendo recalcular los parámetros empíricos anteriores (c, m y n) y los nuevos (a y b).

$$
X=a T_{c}+b
$$

$$
t_{c}=\frac{L^{2}}{\alpha_{0}}\left(a T_{c}+b\right)\left(\frac{1}{B i}+c\right)\left(1+T_{i}\right)^{n}\left(-T_{a}-1\right)^{-m}
$$

Los parámetros fueron calculados regresionando el modelo propuesto con un paquete estadístico (Wilkinson (1990)), con un método de regresión no lineal; los valores correspondientes a las distintas geometrías se presentan en la Tabla 2.6. 
Tabla 2.6. Constantes empíricas de la ec. (2.55)

\begin{tabular}{||lrlrrr||}
\hline \hline Geometría & $\mathrm{a}$ & $\mathrm{b}$ & $\mathrm{c}$ & \multicolumn{1}{c|}{$\mathrm{m}$} & \multicolumn{1}{c|}{$\mathrm{n}$} \\
\hline \hline Placa plana & -1.272 & 65.489 & 0.184 & 1.070 & 0.096 \\
infinita $(\perp)$ & $(0.050)$ & $(2.057)$ & $(0.002)$ & $(0.007)$ & $(0.010)$ \\
& & & & & \\
Placa plana & -0.996 & 63.407 & 0.162 & 1.043 & 0.103 \\
infinita $(\|)$ & $(0.089)$ & $(3.884)$ & $(0.003)$ & $(0.015)$ & $(0.007)$ \\
& & & & & \\
Cilindro & -0.750 & 32.198 & 0.179 & 1.032 & 0.037 \\
infinito & $(0.104)$ & $(3.766)$ & $(0.005)$ & $(0.035)$ & $(0.026)$ \\
Esfera & & & & & \\
& -0.439 & 24.804 & 0.167 & 1.078 & 0.073 \\
& $(0.035)$ & $(1.171)$ & $(0.006)$ & $(0.014)$ & $(0.003)$ \\
\hline
\end{tabular}

N ota: Los valores entre paréntesis corresponden a la desviación estandar de los parámetros estimados.

\subsubsection{CONGELACION ASIMETRICA}

Cuando se congelan hamburguesas, filetes, o alimentos con forma de bloques, paquetes o cajas bajo condiciones de transferencia de calor unidireccional (en congeladores de placas, túneles continuos o discontinuos, congeladores de cinta, etc.) es difícil obtener en ambas superficies del alimento valores idénticos del coeficiente de transferencia calórica $\mathrm{h}$ y/o de la temperatura de refrigerante $\mathrm{T}_{\mathrm{a}}$. A consecuencia de ello, los flujos de calor en las caras opuestas son distintos, causando que el centro térmico se desplace del centro geométrico del producto, moviéndose hacia la cara que presenta menor flujo calórico, a medida que la congelación avanza.

Para poder predecir el tiempo de congelación en esas situaciones, tratamos de adaptar la metodología desarrollada para congelación simétrica, introduciendo pequeñas modificaciones que permitiesen usar las ecs. (2.53) ó (2.55) para los cálculos. La primera dificultad que observamos es que debemos calcular de otro modo la longitud equivalente, que en el caso simétrico tomábamos igual al semiespesor ya que el centro térmico y el geométrico coincidían. 
Definiendo como semiespesor equivalente a la distancia desde una de las caras (la cara 1, por ejemplo) hasta el centro térmico, se propone calcular el tiempo de congelación como si las condiciones operativas fuesen simétricas e iguales a las de la cara 1.

Como se mencionó la posición del centro térmico varía durante la congelación, al principio se ubica en el centro de la pieza, y luego se mueve hacia la cara de menor flujo calórico. Como las ecuaciones de predicción a utilizar regresionan la temperatura final del centro consideramos correcto adoptar la posición final del centro térmico para calcular la longitud característica Leq.

Para evaluar Leq se probaron en primer lugar dos metodologías conocidas:

- De Michelis \& Calvelo (1982) proponen un método basado en la ec. (2.15), dividiendo la congelación en tres períodos. Para cada período deben evaluar la posición del centro térmico.

Para el período de preenfriamiento, los autores encuentran, utilizando un modelo numérico, que el centro térmico no difiere demasiado del centro geométrico, razón por la cual Leq es igual al semiespesor L.

Para el período de atemperado, si la asimetría se origina en distintos coeficientes de transferencia calórica, evalúan Leq de un gráfico elaborado en base a resultados presentados por Uno \& Hayakawa (1979), obtenido de la solución analítica del balance diferencial de energía térmica sin cambio de fase, con propiedades físicas constantes. Cuando la asimetría se origina en las temperaturas externas la posición se obtiene de un gráfico obtenido al resolver numéricamente el balance de energía. Si ocurren simultáneamente ambas causas de asimetría, Leq se calcula con la siguiente expresión:

$$
\frac{L_{e q}}{D}=\left(\frac{L_{e q}}{D}\right)_{T}+-\left[\left(\frac{L_{e q}}{D}\right)_{h}-0.5\right]
$$


donde los semiespesores equivalentes deberán sumarse si ambas causas contribuyen al desplazamiento en un mismo sentido, o restarse si actúan en sentido contrario.

Durante el período de cambio de fase el centro térmico se mueve rápidamente, se propone utilizar un semiespesor equivalente promedio de los correspondientes a los otros dos períodos.

Este modelo de predicción fue probado sólo para calcular la longitud equivalente de la última etapa, la desventaja es que el rango de variación de los parámetros del gráfico que debe utilizarse para calcular (Leq/D)т es demasiado estrecho y no contempla todos los datos experimentales disponibles.

-Pham (1987) considera que el centro térmico es el punto de intersección de dos frentes de congelación que avanzan desde las superficies externas hacia el interior del bloque. Para el cálculo del tiempo de congelación utiliza una ecuación ya presentada (ec. (2.28)) basada en una modificación de la ec. de Plank.

Para el cálculo del semiespesor equivalente plantea que:

$$
L_{e q}=L\left(1+x_{P}\right)
$$

Si $h_{1} \neq h_{2}$ :

$$
x_{P}=\frac{r_{P}-1}{r_{P}+1+B^{\prime} i_{1}} \text { donde } r_{P}=\frac{h_{1}}{h_{2}}
$$

Si $\mathrm{T}_{\mathrm{a} 1} \neq \mathrm{T}_{\mathrm{a} 2}$ :

$$
x_{P}=\frac{\left(1+s_{P}\right)\left(1+\frac{2}{B^{\prime} i}\right)}{\left(1-s_{P}\right)}-\left[\left(\frac{\left(1+s_{P}\right)\left(1+\frac{2}{B^{\prime} i}\right)}{1-s_{P}}\right)^{2}-1+\frac{4}{B^{\prime} i}\right]^{1 / 2}
$$

donde: 


$$
S_{P}=\frac{\left(\frac{\Delta H_{1}^{*}}{\Delta T_{1}}+\frac{\Delta H_{2}^{*}}{\Delta T_{2}}\right)_{\text {Cara } 1}}{\left(\frac{\Delta H_{1}^{*}}{\Delta T_{1}}+\frac{\Delta H_{2}^{*}}{\Delta T_{2}}\right)_{\text {Cara } 2}}
$$

$\Delta \mathrm{H}_{1}{ }^{*}, \Delta \mathrm{H}_{2}{ }^{*}, \Delta \mathrm{T}_{1}$ y $\Delta \mathrm{T}_{2}$ se calculan con las expresiones presentadas con el modelo simétrico (ec. (2.28) y subsiguientes).

Si ocurren ambas asimetrías:

$$
x_{P}=\frac{p_{P}-\left(p_{P}^{2}-\left(1-s_{P}\right) q_{P}\right)^{1 / 2}}{1-s_{P}}
$$

donde:

$$
\begin{aligned}
& p_{P}=\frac{2 s_{P}}{B^{\prime} i_{l}}+\frac{2 r_{P}}{B^{\prime} i_{1}}+s_{P}+1 \\
& q_{P}=\frac{-4 s_{P}}{B^{\prime} i_{1}}+\frac{4 r_{P}}{B^{\prime} i_{l}}-s_{P}+1
\end{aligned}
$$

Este esquema de cálculo de la longitud equivalente fue ensayado, calculando el tiempo de congelación con la ec. (2.53). Comparando los valores predichos con resultados experimentales, en todos los casos se subpredecía levemente el tiempo de congelación. Además el cálculo de la longitud equivalente resulta demasiado complicado para un método aproximado que busca utilizar una única ecuación simple para el cálculo de tc.

La solución elegida consiste en una tercera alternativa: como ya mencionamos adoptamos como Leq la distancia hasta el centro térmico calculada al final de la congelación, cuando ya se ha producido el cambio de fase. Si se presentan distintos coeficientes de transferencia calórica utilizamos el gráfico elaborado en base a los resultados de Uno \& Hayakawa (reproducido en la Figura

Cap. 2-40 
2.9).

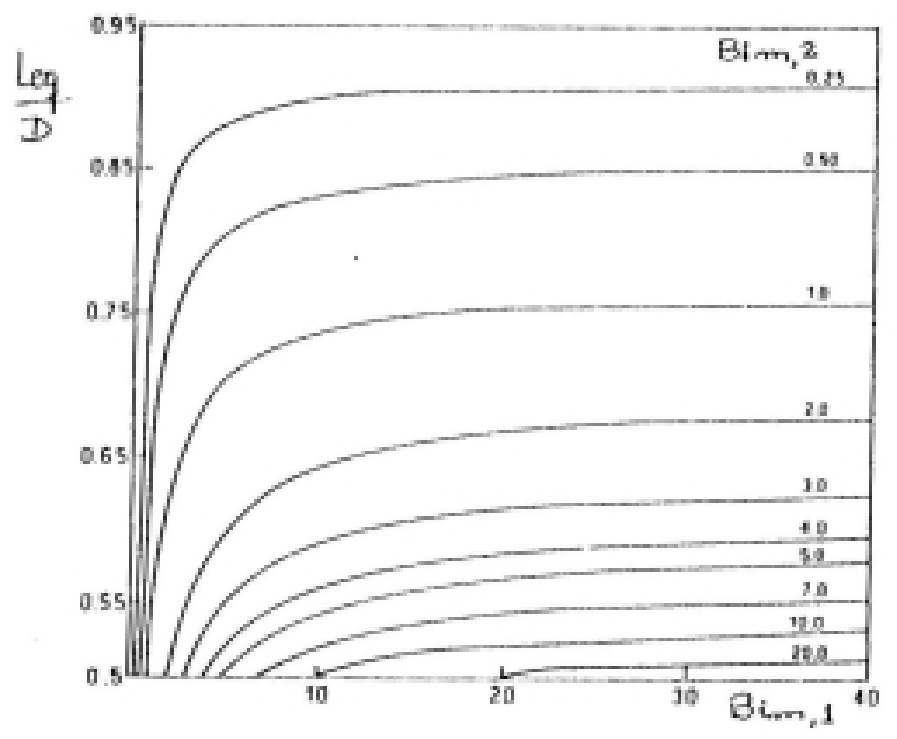

Figura 2.9. Semiespesor equivalente en función de los números de Biot.

Cuando las temperaturas externas difieren, se calcula Leq a partir de la solución analítica exacta para el caso de transferencia de calor sin cambio de fase, utilizando propiedades constantes e iguales a las del producto congelado. Esta resulta en una serie infinita, pero a tiempos largos puede trabajarse con un único término:

$$
T=C_{I} r+C_{2}+A_{I} e^{-\lambda_{1}^{2} \alpha_{s} t}\left(\lambda_{1} \cos \left(\lambda_{1} r\right)+\frac{B^{\prime} i}{D} \operatorname{sen}\left(\lambda_{1} r\right)\right)
$$

donde:

$$
\begin{gathered}
A_{l}=\frac{2}{\lambda_{1} D+\operatorname{sen}\left(\lambda_{1} D\right) \cos \left(\lambda_{1} D\right)}\left[\left(T_{i}-C_{2}\right) \operatorname{sen}\left(\lambda_{1} D\right)+\frac{C_{l}}{\lambda_{l}}\left(\lambda_{1} D \operatorname{sen}\left(\lambda_{1} D\right)+\cos \left(\lambda_{l} D\right)-1\right)\right] \\
C_{1}=\frac{B^{\prime} i\left(T_{a 2}-T_{a 1}\right)}{D\left(2+B^{\prime} i\right)} \\
C_{2}=\frac{T_{a l}\left(1+B^{\prime} i\right)+T_{a 2}}{2+B^{\prime} i}
\end{gathered}
$$

$\lambda_{1}$ es la primera raíz de la siguiente ecuación trascendental:

$$
\lambda_{1} L \operatorname{tg}\left(\lambda_{1} L\right)=\frac{B^{\prime} i}{2}
$$


La posición del centro térmico se encuentra derivando la ec. (2.61) respecto de la posición, e igualando a cero ya que en ese punto la temperatura es máxima. Esta operación conduce a que el centro térmico se encuentra en la raíz de la siguiente igualdad (ec. (2.62)):

$$
C_{l}+A_{l} e^{-\lambda_{I}^{2} \alpha_{s} t} \lambda_{1}\left(-\lambda_{1} \operatorname{sen}\left(\lambda_{1} r\right)+\frac{B^{\prime} i}{D} \cos \left(\lambda_{1} r\right)\right)=0
$$

Las raíces de (2.62) fueron calculadas para un amplio rango de valores de Bi' y C1. Para facilitar el uso de estos resultados, se construyó el nomograma de la Figura 2.10.

Para utilizar el nomograma, debe ubicarse en la región derecha del mismo, el punto que resulta de la intersección de ambas temperaturas externas, Ta1 y Ta2. Dicho valor es la asíntota de las curvas de la izquierda de la Figura ((Leq/D) vs. Bi'). Con el valor de la asíntota se define la curva a usar para tomar el valor de Leq/D con el número de Biot correspondiente.

Si ocurren ambas asimetrías, se suma el efecto de ambos según la ec. (2.56). 


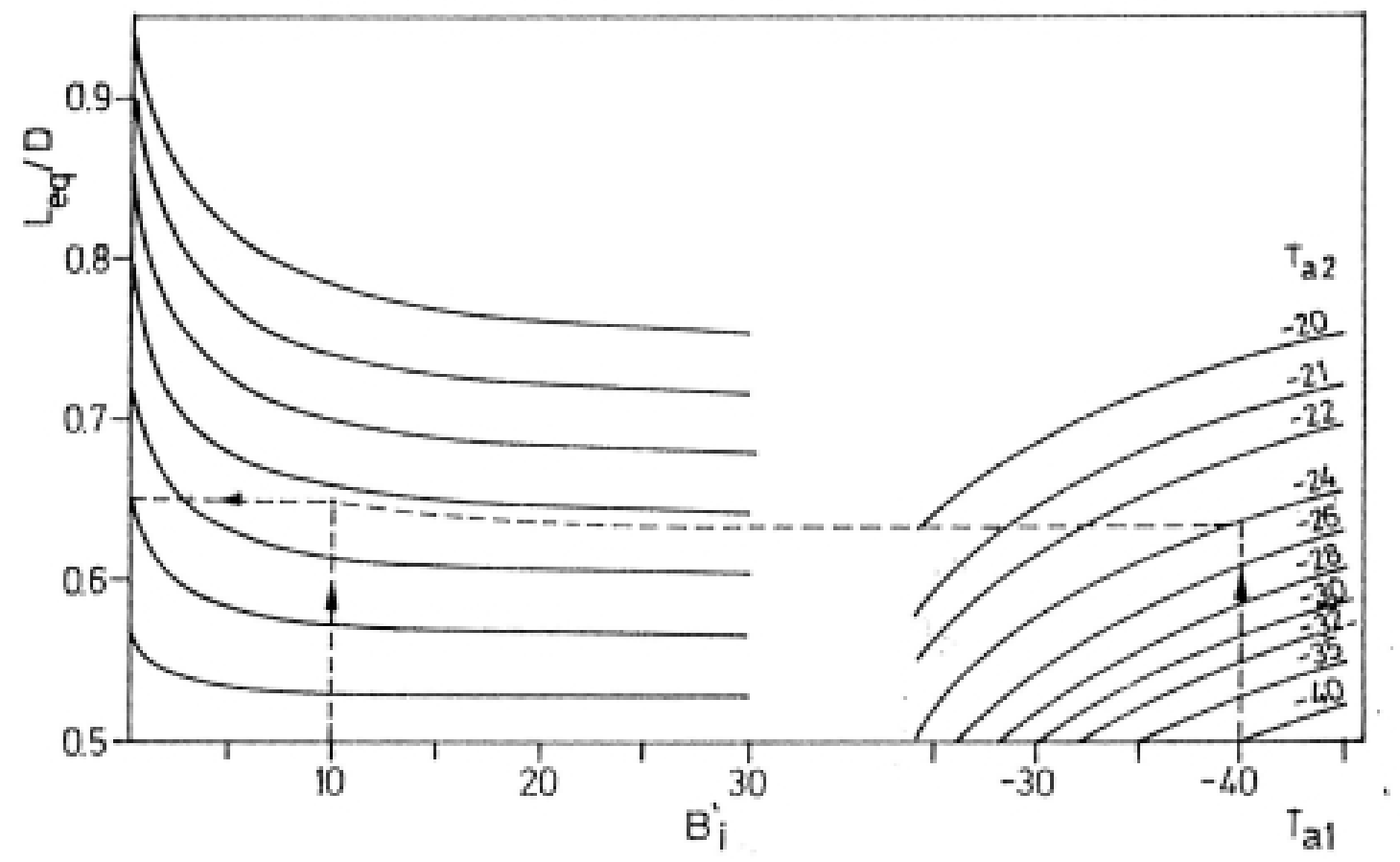

Figura 2.10. Semiespesor equivalente, en función de las temperaturas externas y del número de Biot.

\subsubsection{RESULTADOS}

Para verificar la validez de los métodos de predicción presentados, deben compararse los tiempos calculados con tiempos de congelación experimentales. Una práctica común en estos casos es tomar todos los resultados experimentales que han sido publicados y contrastarlos con los valores predichos.

Para demostrar que el método desarrollado puede aplicarse a alimentos de distinta composición y estructura, se tomaron los datos correspondientes a distintos tipos de alimentos: carnes, pescados, vegetales, frutas, sustancias de prueba.

En la Tabla 2.7 se detallan los valores de las propiedades físicas utilizados en los cálculos. En algunos casos se empleó el valor presentado por los autores, en otros se adoptaron valores experimentales o calculados en base a ecuaciones de predicción existentes (Singh \& Lund (1984), Sanz y col. $(1987,1989))$. Cabe aclarar 
que en la actualidad la falta de precisión de los métodos muchas veces se debe a que no se conocen con exactitud los valores de las propiedades termofísicas; además un mismo juego de datos puede generar resultados con mayor o menor precisión de acuerdo a los valores de propiedades físicas utilizadas.

Cap. 2-44 
Tabla 2.7. Propiedades físicas de los alimentos frescos, a temperatura ambiente.

\begin{tabular}{|c|c|c|c|c|c|}
\hline Alimento & $\begin{array}{c}\rho_{0} \\
\left(\mathrm{~kg} / \mathrm{m}^{3}\right) \\
\end{array}$ & $\begin{array}{c}\mathrm{C}_{\mathrm{p} 0} \\
\mathrm{~J} /\left(\mathrm{kg}^{\circ} \mathrm{C}\right) \\
\end{array}$ & $\begin{array}{c}\mathrm{k}_{0} \\
\mathrm{~W} /\left(\mathrm{m}^{\circ} \mathrm{C}\right) \\
\end{array}$ & $\mathrm{Y}_{0}$ & $\begin{array}{l}\alpha_{0} 10^{7} \\
\left(\mathrm{~m}^{2} / \mathrm{s}\right) \\
\end{array}$ \\
\hline Tilosa & 1006.0 & 3653.3 & 0.550 & 0.77 & 1.500 \\
\hline Gel de agar & 1006.4 & 4180.0 & 0.564 & 0.97 & 1.340 \\
\hline Carne vacuna $(\perp)$ & 1053.0 & 3475.0 & 0.480 & 0.74 & 1.310 \\
\hline Carne vacuna $(\|)$ & 1053.0 & 3475.0 & 0.510 & 0.74 & 1.390 \\
\hline \multirow[t]{3}{*}{ Carne picada } & 1041.7 & 3244.7 & 0.450 & 0.63 & 1.330 \\
\hline & 1000.0 & 3310.5 & 0.434 & 0.68 & 1.310 \\
\hline & 1000.0 & 3650.0 & 0.500 & 0.74 & 1.370 \\
\hline Cordero & 1000.0 & 3487.0 & 0.495 & 0.74 & 1.240 \\
\hline Albóndigas & 973.0 & 3364.8 & 0.441 & 0.70 & 1.347 \\
\hline Hígado de cerdo & 1057.0 & 3370.0 & 0.440 & 0.70 & 1.240 \\
\hline Carpa & 1035.5 & 3457.3 & 0.480 & 0.70 & 1.340 \\
\hline Tiburón & 1054.0 & 3600.0 & 0.453 & 0.85 & 1.190 \\
\hline Bacalao & 1000.0 & 3720.0 & 0.470 & 0.83 & 1.260 \\
\hline Sardina & 770.0 & 3440.0 & 0.464 & 0.73 & 1.750 \\
\hline Caballa & 1003.0 & 3470.0 & 0.463 & 0.77 & 1.330 \\
\hline Camarón & 1087.0 & 3500.0 & 0.500 & 0.80 & 1.310 \\
\hline Puré de papas & 1089.3 & 3360.0 & 0.530 & 0.83 & 1.450 \\
\hline Manzana & 797.0 & 3760.0 & 0.393 & 0.80 & 1.310 \\
\hline
\end{tabular}

\subsubsection{RESULTADOS DE CONGELACION SIMETRICA}

Las Tablas 2.8 a 2.19 presentan en detalle 200 medidas experimentales extractadas de bibliografía, correspondientes a placa plana infinita, con transferencia de energía perpendicular a las fibras. En cada caso se detallan el 
semiespesor L, las condiciones operativas y el tiempo de congelación experimental, $t_{\text {cexp. }}$ Cada tabla corresponde a los resultados obtenidos para un mismo producto por un mismo autor. En la penúltima columna se presenta el tiempo de congelación calculado con la ec. (2.55).

En la Figura 2.11 ( $\mathrm{a}$ y b) se han graficado los tiempos de congelación predichos vs. los valores experimentales, para los distintos tipos de productos. Por otra parte en la Figura 2.12 ( $\mathrm{a}$ y b) la distinción se hace en función de que las condiciones operativas (coeficiente de transferencia calórica, temperatura externa o temperatura inicial) estén dentro o fuera del rango detallado en la Tabla 2.4, barrido con el modelo numérico. La ec. (2.55) es estrictamente válida para el mencionado rango de condiciones, pero la Figura 2.12 muestra que la misma puede utilizarse fuera de dicho margen sin perder precisión. Las Tablas 2.20 y 2.21 corresponden a placa plana infinita, con transferencia de calor paralela a las fibras, en este caso se cuenta con pocos valores experimentales (13 en total), todos de carne vacuna. Análogamente la Figura 2.13 muestra los tiempos predichos vs. los tiempos experimentales, diferenciados en base a las condiciones operativas.

Las Tablas 2.22 y 2.23 corresponden a cilindro infinito, de tilosa y camarón. Para esta geometría se cuenta con 36 datos experimentales de congelación. Similarmente a placa plana, las Figuras 2.14 y 2.15 diferencian los valores graficados por tipo de producto y por rango de condiciones operativas, respectivamente. Análogamente, las Tablas 2.24 a 2.26 corresponden a esfera, contando con 114 valores experimentales. En la Figura 2.16 ( $\mathrm{a} \mathrm{y} \mathrm{b}$ ) se han graficado los tiempos predichos vs. los experimentales, diferenciados por tipo de producto. A su vez en la Figura 2.17 ( $a$ y b) los valores se han diferenciado por sus condiciones operativas. 
Tabla 2.8. Tiempos de congelación, experimentales y calculados. Placa plana, tilosa (Cleland \& Earle, 1977).

\begin{tabular}{|c|c|c|c|c|c|c|c|c|}
\hline $\begin{array}{c}\mathrm{L} \\
(\mathrm{cm})\end{array}$ & $\begin{array}{c}\mathrm{h} \\
\mathrm{W} /\left(\mathrm{m}^{2 \circ} \mathrm{C}\right. \\
)\end{array}$ & $\mathrm{Bi}$ & $\begin{array}{c}\mathrm{T}_{\mathrm{a}} \\
\left({ }^{\circ} \mathrm{C}\right)\end{array}$ & $\begin{array}{c}\mathrm{T}_{\mathrm{i}} \\
\left({ }^{\circ} \mathrm{C}\right)\end{array}$ & $\begin{array}{c}\mathrm{T}_{\mathrm{c}} \\
\left({ }^{\circ} \mathrm{C}\right)\end{array}$ & $\begin{array}{c}t_{\text {cexp }} \\
(\mathrm{min})\end{array}$ & $\begin{array}{c}\mathrm{t}_{\text {cpre }} \\
\text { (min) }\end{array}$ & $\begin{array}{c}\mathrm{e} \\
(\%)\end{array}$ \\
\hline 5.00 & 320.0 & 26.23 & -20.0 & 28.0 & -10.0 & 256.8 & 280.7 & 9.32 \\
\hline 5.00 & 310.0 & 25.41 & -23.0 & 3.0 & -10.0 & 207.6 & 199.4 & -3.94 \\
\hline 3.60 & 410.0 & 24.20 & -22.0 & 3.0 & -10.0 & 115.2 & 109.5 & -4.92 \\
\hline 3.60 & 330.0 & 19.48 & -24.5 & 10.5 & -10.0 & 109.2 & 111.9 & 2.44 \\
\hline 3.60 & 90.0 & 5.31 & -21.7 & 11.0 & -10.0 & 181.2 & 197.6 & 9.06 \\
\hline 3.60 & 51.9 & 3.06 & -40.0 & 30.0 & -10.0 & 157.2 & 148.6 & -5.45 \\
\hline 3.60 & 51.9 & 3.06 & -20.0 & 30.0 & -10.0 & 288.0 & 320.8 & 11.40 \\
\hline 3.60 & 51.9 & 3.06 & -40.0 & 10.0 & -10.0 & 133.2 & 134.6 & 1.02 \\
\hline 3.60 & 51.9 & 3.06 & -20.0 & 10.0 & -10.0 & 270.0 & 290.5 & 7.58 \\
\hline 3.60 & 21.6 & 1.27 & -40.0 & 30.0 & -10.0 & 284.4 & 276.9 & -2.63 \\
\hline 3.60 & 21.6 & 1.27 & -20.0 & 30.0 & -10.0 & 537.6 & 597.8 & 11.20 \\
\hline 3.60 & 21.6 & 1.27 & -40.0 & 10.0 & -10.0 & 241.2 & 250.7 & 3.94 \\
\hline 3.60 & 21.6 & 1.27 & -20.0 & 10.0 & -10.0 & 477.6 & 541.2 & 13.32 \\
\hline 3.60 & 16.7 & 0.99 & -26.7 & 28.0 & -10.0 & 510.0 & 530.0 & 3.93 \\
\hline 3.60 & 13.6 & 0.80 & -26.0 & 3.0 & -10.0 & 505.2 & 53.1 & 6.31 \\
\hline 2.43 & 410.0 & 16.30 & -21.0 & 11.0 & -10.0 & 60.0 & 62.9 & 4.90 \\
\hline 2.43 & 360.0 & 14.31 & -23.5 & 3.0 & -10.0 & 52.8 & 51.5 & -2.40 \\
\hline 2.43 & 90.0 & 3.58 & -22.0 & 34.5 & -10.0 & 122.4 & 120.8 & -1.33 \\
\hline 2.43 & 90.0 & 3.58 & -24.3 & 13.7 & -10.0 & 92.4 & 99.3 & 7.46 \\
\hline 2.43 & 30.6 & 1.22 & -30.0 & 20.0 & -10.0 & 164.4 & 172.5 & 4.94 \\
\hline 2.43 & 30.6 & 1.22 & -30.0 & 20.0 & -10.0 & 164.4 & 172.5 & 4.94 \\
\hline 2.43 & 30.6 & 1.22 & -30.0 & 20.0 & -10.0 & 164.4 & 172.5 & 4.94 \\
\hline 2.43 & 30.6 & 1.22 & -30.0 & 20.0 & -10.0 & 162.0 & 172.5 & 6.49 \\
\hline 2.43 & 30.6 & 1.22 & -30.0 & 20.0 & -10.0 & 159.6 & 172.5 & 8.09 \\
\hline 2.43 & 16.7 & 0.66 & -25.3 & 15.3 & -10.0 & 315.6 & 339.1 & 7.45 \\
\hline 2.43 & 13.6 & 0.54 & -29.5 & 28.6 & -10.0 & 349.2 & 363.5 & 4.11 \\
\hline 2.43 & 13.6 & 0.54 & -26.0 & 3.0 & -10.0 & 320.4 & 345.1 & 7.72 \\
\hline 1.25 & 430.0 & 8.81 & -19.0 & 3.0 & -10.0 & 20.4 & 20.2 & -1.21 \\
\hline 1.25 & 400.0 & 8.20 & -22.0 & 21.6 & -10.0 & 19.2 & 20.7 & 7.92 \\
\hline 1.25 & 90.0 & 1.84 & -24.0 & 28.0 & -10.0 & 40.8 & 44.1 & 8.01 \\
\hline 1.25 & 90.0 & 1.84 & -20.0 & 11.0 & -10.0 & 44.4 & 49.7 & 11.87 \\
\hline 1.25 & 51.9 & 1.06 & -40.0 & 30.0 & -10.0 & 38.4 & 38.7 & 0.65 \\
\hline 1.25 & 51.9 & 1.06 & -20.0 & 30.0 & -10.0 & 75.6 & 83.4 & 10.36 \\
\hline 1.25 & 51.9 & 1.06 & -40.0 & 10.0 & -10.0 & 33.6 & 35.0 & 4.14 \\
\hline 1.25 & 51.9 & 1.06 & -20.0 & 10.0 & -10.0 & 67.2 & 75.5 & 12.40 \\
\hline 1.25 & 21.6 & 0.44 & -40.0 & 30.0 & -10.0 & 85.2 & 83.2 & -2.35 \\
\hline 1.25 & 21.6 & 0.44 & -20.0 & 30.0 & -10.0 & 164.4 & 179.6 & 9.24 \\
\hline 1.25 & 21.6 & 0.44 & -40.0 & 10.0 & -10.0 & 75.6 & 75.3 & -0.37 \\
\hline 1.25 & 21.6 & 0.44 & -20.0 & 10.0 & -10.0 & 146.4 & 162.6 & 11.06 \\
\hline 1.25 & 16.7 & 0.34 & -26.0 & 28.7 & -10.0 & 160.8 & 169.2 & 5.24 \\
\hline 1.25 & 16.7 & 0.34 & -24.5 & 3.0 & -10.0 & 144.0 & 149.2 & 3.58 \\
\hline 1.25 & 13.6 & 0.28 & -25.3 & 22.0 & -10.0 & 186.0 & 206.5 & 11.02 \\
\hline 1.25 & 13.6 & 0.28 & -29.6 & 5.8 & -10.0 & 139.2 & 154.3 & 10.85 \\
\hline
\end{tabular}


Tabla 2.9. Tiempos de congelación, experimentales y calculados. Placa plana, tilosa (Pham \& Willix, 1990).

\begin{tabular}{|c|c|c|c|c|c|c|c|c|}
\hline $\begin{array}{c}\mathrm{L} \\
(\mathrm{cm})\end{array}$ & $\begin{array}{c}\mathrm{h} \\
\mathrm{W} /\left(\mathrm{m}^{2 \circ} \mathrm{C}\right. \\
)\end{array}$ & $\mathrm{Bi}$ & $\begin{array}{c}\mathrm{T}_{\mathrm{a}} \\
\left({ }^{\circ} \mathrm{C}\right)\end{array}$ & $\begin{array}{c}\mathrm{T}_{\mathrm{i}} \\
\left({ }^{\circ} \mathrm{C}\right)\end{array}$ & $\begin{array}{c}\mathrm{T}_{\mathrm{c}} \\
\left({ }^{\circ} \mathrm{C}\right)\end{array}$ & $\begin{array}{c}t_{\text {cexp }} \\
(\mathrm{min})\end{array}$ & $\begin{array}{c}\mathrm{t}_{\text {cpre }} \\
\text { (min) }\end{array}$ & $\begin{array}{c}\mathrm{e} \\
(\%)\end{array}$ \\
\hline 3.79 & 306.4 & 23.70 & -39.5 & 18.3 & -18.0 & 91.2 & 87.2 & -4.35 \\
\hline 7.53 & 326.7 & 50.17 & -38.5 & 18.3 & -18.0 & 330.6 & 315.5 & -4.57 \\
\hline 3.83 & 81.6 & 6.37 & -40.8 & 18.2 & -18.0 & 135.0 & 133.4 & -1.16 \\
\hline 7.49 & 80.2 & 12.25 & -39.7 & 18.7 & -18.0 & 403.2 & 403.9 & 0.17 \\
\hline 3.82 & 25.3 & 1.97 & -39.7 & 18.2 & -18.0 & 279.6 & 286.7 & 2.55 \\
\hline 7.47 & 23.5 & 3.58 & -40.6 & 18.5 & -18.0 & 693.6 & 707.5 & 2.00 \\
\hline 3.80 & 246.5 & 19.09 & -39.6 & 17.4 & -18.0 & 95.4 & 91.1 & -4.50 \\
\hline 7.39 & 199.6 & 30.10 & -39.2 & 18.9 & -18.0 & 323.4 & 320.8 & -0.80 \\
\hline 3.78 & 259.6 & 20.03 & -23.9 & 18.1 & -18.0 & 166.8 & 156.8 & -5.99 \\
\hline 7.36 & 94.3 & 14.15 & -24.5 & 19.1 & -18.0 & 612.0 & 636.5 & 4.01 \\
\hline 3.79 & 80.9 & 6.25 & -24.8 & 18.5 & -18.0 & 229.2 & 229.0 & -0.09 \\
\hline 7.31 & 44.7 & 6.66 & -25.9 & 19.0 & -18.0 & 741.0 & 789.6 & 6.56 \\
\hline 4.03 & 23.3 & 1.92 & -22.9 & 18.8 & -18.0 & 624.0 & 601.6 & -3.59 \\
\hline 7.26 & 19.1 & 2.83 & -23.9 & 19.8 & -18.0 & 1393.8 & 1409.4 & 1.12 \\
\hline 4.00 & 221.5 & 18.08 & -25.0 & 19.3 & -18.0 & 181.2 & 172.2 & -4.97 \\
\hline 7.69 & 117.8 & 18.48 & -26.2 & 2.2 & -18.0 & 511.8 & 502.5 & -1.81 \\
\hline 3.94 & 241.8 & 19.44 & -39.7 & 1.1 & -18.0 & 89.4 & 79.2 & -11.45 \\
\hline 7.53 & 144.4 & 22.18 & -39.4 & 2.3 & -18.0 & 305.4 & 295.2 & -3.35 \\
\hline 3.98 & 64.8 & 5.26 & -40.8 & 2.5 & -18.0 & 135.0 & 135.3 & 0.23 \\
\hline 7.51 & 56.6 & 8.67 & -40.2 & 3.3 & -18.0 & 379.2 & 393.6 & 3.79 \\
\hline 3.94 & 22.9 & 1.84 & -40.1 & 1.9 & -18.0 & 260.4 & 264.7 & 1.66 \\
\hline 7.47 & 19.1 & 2.91 & -41.0 & 1.9 & -18.0 & 652.8 & 666.9 & 2.16 \\
\hline 3.92 & 106.6 & 8.52 & -40.3 & 2.4 & -18.0 & 108.0 & 105.2 & -2.59 \\
\hline 7.70 & 127.2 & 19.99 & -39.1 & 2.6 & -18.0 & 326.4 & 321.7 & -1.45 \\
\hline 3.91 & 122.3 & 9.76 & -26.2 & 1.0 & -18.0 & 162.0 & 151.9 & -6.23 \\
\hline 7.50 & 64.1 & 9.80 & -24.4 & 3.4 & -18.0 & 634.8 & 650.6 & 2.49 \\
\hline 3.91 & 64.5 & 5.14 & -25.3 & 2.3 & -18.0 & 223.8 & 222.4 & -0.62 \\
\hline 7.63 & 59.2 & 9.22 & -24.8 & 2.1 & -18.0 & 652.2 & 655.8 & 0.56 \\
\hline 3.89 & 23.1 & 1.83 & -25.5 & 2.1 & -18.0 & 431.4 & 429.6 & -0.42 \\
\hline 7.58 & 18.7 & 2.89 & -25.5 & 2.2 & -18.0 & 1154.4 & 1176.5 & 1.91 \\
\hline 3.89 & 113.9 & 9.04 & -24.1 & 1.5 & -18.0 & 185.4 & 173.7 & -6.29 \\
\hline 7.50 & 55.3 & 8.46 & -25.1 & 2.1 & -18.0 & 632.4 & 646.8 & 2.27 \\
\hline
\end{tabular}

Cap. $2-48$ 
Tabla 2.10. Tiempos de congelación, experimentales y calculados. Placa plana, tilosa (Hung \& Thompson, 1983).

\begin{tabular}{|c|c|c|c|c|c|c|c|c|}
\hline $\begin{array}{c}\mathrm{L} \\
(\mathrm{cm})\end{array}$ & $\begin{array}{c}\mathrm{h} \\
\mathrm{W} /\left(\mathrm{m}^{2 \circ} \mathrm{C}\right.\end{array}$ & $\mathrm{Bi}$ & $\begin{array}{c}\mathrm{T}_{\mathrm{a}} \\
\left({ }^{\circ} \mathrm{C}\right)\end{array}$ & $\begin{array}{c}\mathrm{T}_{\mathrm{i}} \\
\left({ }^{\circ} \mathrm{C}\right)\end{array}$ & $\begin{array}{c}\mathrm{T}_{\mathrm{c}} \\
\left({ }^{\circ} \mathrm{C}\right)\end{array}$ & $\begin{array}{c}t_{\text {cexp }} \\
(\mathrm{min})\end{array}$ & $\begin{array}{c}\mathrm{t}_{\text {cpre }} \\
\text { (min) }\end{array}$ & $\begin{array}{c}\mathrm{e} \\
(\%)\end{array}$ \\
\hline 0.65 & 9.0 & 0.11 & -19.9 & 5.1 & -18.0 & 184.0 & 203.8 & 10.75 \\
\hline 1.40 & 11.5 & 0.29 & -25.0 & 18.3 & -18.0 & 302.0 & 307.1 & 1.69 \\
\hline 0.65 & 9.0 & 0.11 & -30.1 & 2.9 & -18.0 & 122.0 & 123.0 & 0.83 \\
\hline 2.40 & 11.0 & 0.48 & -30.1 & 3.7 & -18.0 & 374.0 & 403.9 & 7.98 \\
\hline 0.60 & 11.0 & 0.12 & -30.1 & 29.6 & -18.0 & 138.0 & 113.5 & -17.75 \\
\hline 2.35 & 11.0 & 0.47 & -20.4 & 30.2 & -18.0 & 791.0 & 730.6 & -7.63 \\
\hline 1.45 & 108.0 & 2.85 & -29.3 & 3.5 & -18.0 & 33.0 & 35.7 & 8.19 \\
\hline 0.55 & 110.0 & 1.10 & -32.4 & 26.3 & -18.0 & 12.5 & 11.2 & -10.72 \\
\hline 2.25 & 108.0 & 4.42 & -29.3 & 29.6 & -18.0 & 87.5 & 79.2 & -9.46 \\
\hline 0.60 & 108.0 & 1.18 & -30.9 & 16.8 & -18.0 & 13.3 & 12.7 & -4.58 \\
\hline 2.40 & 108.0 & 4.71 & -29.4 & 4.2 & -18.0 & 73.5 & 73.1 & -0.49 \\
\hline 0.55 & 106.0 & 1.06 & -25.7 & 4.4 & -18.0 & 12.7 & 12.7 & 0.29 \\
\hline 0.50 & 104.0 & 0.95 & -20.3 & 30.2 & -18.0 & 20.0 & 17.9 & -10.68 \\
\hline 2.40 & 104.0 & 4.54 & -20.0 & 4.5 & -18.0 & 111.0 & 115.4 & 3.95 \\
\hline 0.55 & 104.0 & 1.04 & -20.1 & 4.4 & -18.0 & 16.0 & 17.0 & 6.50 \\
\hline 0.55 & 69.0 & 0.69 & -30.6 & 3.8 & -18.0 & 14.8 & 15.0 & 1.57 \\
\hline 2.20 & 68.0 & 2.72 & -25.6 & 18.0 & -18.0 & 116.0 & 113.0 & -2.58 \\
\hline 1.35 & 68.0 & 1.67 & -24.9 & 30.9 & -18.0 & 74.0 & 65.5 & -11.51 \\
\hline 1.50 & 67.0 & 1.83 & -20.1 & 17.4 & -18.0 & 93.0 & 91.0 & -2.14 \\
\hline 1.44 & 68.0 & 1.77 & -25.0 & 17.3 & -18.0 & 61.8 & 66.7 & 7.87 \\
\hline 1.35 & 68.0 & 1.67 & -24.7 & 17.3 & -18.0 & 62.0 & 62.6 & 1.03 \\
\hline 1.35 & 68.0 & 1.67 & -25.2 & 17.4 & -18.0 & 60.0 & 61.3 & 2.15 \\
\hline 1.40 & 68.0 & 1.73 & -25.1 & 17.9 & -18.0 & 67.0 & 64.6 & -3.64 \\
\hline
\end{tabular}

Tabla 2.11. Tiempos de congelación, experimentales y calculados. Placa plana, cordero (Bazán \& Mascheroni, 1984).

\begin{tabular}{|c|c|c|c|c|c|c|c|c|}
\hline $\begin{array}{c}\mathrm{L} \\
(\mathrm{cm})\end{array}$ & $\begin{array}{c}\mathrm{h} \\
\mathrm{W} /\left(\mathrm{m}^{2 \circ} \mathrm{C}\right. \\
) \\
\end{array}$ & $\mathrm{Bi}$ & $\begin{array}{c}\mathrm{T}_{\mathrm{a}} \\
\left({ }^{\circ} \mathrm{C}\right)\end{array}$ & $\begin{array}{c}\mathrm{T}_{\mathrm{i}} \\
\left({ }^{\circ} \mathrm{C}\right)\end{array}$ & $\begin{array}{c}\mathrm{T}_{\mathrm{c}} \\
\left({ }^{\circ} \mathrm{C}\right)\end{array}$ & $\begin{array}{c}t_{\text {cexp }} \\
\text { (min) }\end{array}$ & $\begin{array}{c}\mathrm{t}_{\text {cpre }} \\
\text { (min) }\end{array}$ & $\begin{array}{c}\mathrm{e} \\
(\%)\end{array}$ \\
\hline 3.00 & 79.5 & 4.82 & -37.0 & 10.6 & -18.0 & 114.0 & 114.5 & 0.43 \\
\hline 3.00 & 99.6 & 6.04 & -40.2 & 9.6 & -18.0 & 88.2 & 92.7 & 5.06 \\
\hline 3.00 & 120.5 & 7.30 & -33.2 & 10.3 & -18.0 & 115.2 & 105.7 & -8.29 \\
\hline 3.00 & 102.9 & 6.24 & -39.9 & 9.4 & -18.0 & 99.0 & 91.7 & -7.36 \\
\hline
\end{tabular}

Cap. 2-49 
Tabla 2.12. Tiempos de congelación, experimentales y calculados. Placa plana, carne vacuna (Mascheroni \& Calvelo, 1982).

\begin{tabular}{|c|c|c|c|c|c|c|c|c|}
\hline $\begin{array}{c}\mathrm{L} \\
(\mathrm{cm})\end{array}$ & $\begin{array}{c}\mathrm{h} \\
\mathrm{W} /\left(\mathrm{m}^{2 \circ} \mathrm{C}\right. \\
) \\
\end{array}$ & $\mathrm{Bi}$ & $\begin{array}{c}\mathrm{T}_{\mathrm{a}} \\
\left({ }^{\circ} \mathrm{C}\right)\end{array}$ & $\begin{array}{c}\mathrm{T}_{\mathrm{i}} \\
\left({ }^{\circ} \mathrm{C}\right)\end{array}$ & $\begin{array}{c}\mathrm{T}_{\mathrm{c}} \\
\left({ }^{\circ} \mathrm{C}\right)\end{array}$ & $\begin{array}{c}\mathrm{t}_{\mathrm{cexp}} \\
(\mathrm{min})\end{array}$ & $\begin{array}{c}\mathrm{t}_{\text {cpre }} \\
\text { (min) }\end{array}$ & $\begin{array}{c}\mathrm{e} \\
(\%)\end{array}$ \\
\hline 3.00 & 168.5 & 10.53 & -38.7 & 18.3 & -18.0 & 78.0 & 77.2 & -1.06 \\
\hline 3.10 & 217.7 & 14.06 & -36.2 & 14.1 & -18.0 & 83.8 & 79.2 & -5.47 \\
\hline 3.00 & 147.0 & 9.19 & -33.3 & 18.0 & -18.0 & 91.0 & 95.4 & 4.88 \\
\hline 3.10 & 161.7 & 10.44 & -39.0 & 17.0 & -18.0 & 80.0 & 81.4 & 1.74 \\
\hline 3.00 & 156.3 & 9.77 & -44.2 & 12.0 & -18.0 & 64.0 & 65.9 & 3.02 \\
\hline 3.00 & 137.9 & 8.62 & -39.0 & 19.7 & -18.0 & 70.0 & 82.9 & 18.37 \\
\hline 3.00 & 138.1 & 8.63 & -44.7 & 11.4 & -18.0 & 68.0 & 67.9 & -0.17 \\
\hline 3.00 & 143.5 & 8.97 & -40.6 & 19.9 & -18.0 & 73.5 & 78.2 & 6.33 \\
\hline 3.00 & 88.1 & 5.51 & -39.9 & 17.8 & -18.0 & 94.2 & 97.6 & 3.57 \\
\hline 3.00 & 72.3 & 4.52 & -27.3 & 22.0 & -18.0 & 148.0 & 167.6 & 13.26 \\
\hline
\end{tabular}

Tabla 2.13. Tiempos de congelación, experimentales y calculados. Placa plana, carne picada, $\mathrm{Y}_{0}=0.74$ (Cleland \& Earle, 1977).

\begin{tabular}{|c|c|c|c|c|c|c|c|c|}
\hline $\begin{array}{c}\mathrm{L} \\
(\mathrm{cm})\end{array}$ & $\begin{array}{c}\mathrm{h} \\
\mathrm{W} /\left(\mathrm{m}^{2 \circ} \mathrm{C}\right. \\
)\end{array}$ & $\mathrm{Bi}$ & $\begin{array}{c}\mathrm{T}_{\mathrm{a}} \\
\left({ }^{\circ} \mathrm{C}\right)\end{array}$ & $\begin{array}{c}\mathrm{T}_{\mathrm{i}} \\
\left({ }^{\circ} \mathrm{C}\right)\end{array}$ & $\begin{array}{c}\mathrm{T}_{\mathrm{c}} \\
\left({ }^{\circ} \mathrm{C}\right)\end{array}$ & $\begin{array}{c}t_{c e x p} \\
(\mathrm{~min})\end{array}$ & $\begin{array}{c}\mathrm{t}_{\text {cpre }} \\
(\mathrm{min})\end{array}$ & $\begin{array}{c}\mathrm{e} \\
(\%)\end{array}$ \\
\hline 3.60 & 220.0 & 15.84 & -25.0 & 28.0 & -10.0 & 139.0 & 140.4 & 1.03 \\
\hline 3.60 & 51.9 & 3.74 & -23.9 & 3.0 & -10.0 & 230.0 & 223.1 & -2.99 \\
\hline 2.40 & 90.0 & 4.32 & -25.4 & 30.0 & -10.0 & 101.0 & 103.8 & 2.73 \\
\hline 2.40 & 21.6 & 1.04 & -28.4 & 3.0 & -10.0 & 198.0 & 208.1 & 5.12 \\
\hline 1.25 & 30.6 & 0.77 & -26.7 & 28.6 & -10.0 & 92.0 & 95.1 & 3.41 \\
\hline 1.25 & 16.7 & 0.42 & -28.8 & 16.6 & -10.0 & 140.0 & 143.9 & 2.80 \\
\hline
\end{tabular}

Tabla 2.14. Tiempos de congelación, experimentales y calculados. Placa plana, carne picada, $\mathrm{Y}_{0}=0.63$ (Hung \& Thompson, 1983).

\begin{tabular}{|c|c|c|c|c|c|c|c|c|}
\hline $\begin{array}{c}\mathrm{L} \\
(\mathrm{cm})\end{array}$ & $\begin{array}{c}\mathrm{h} \\
\mathrm{W} /\left(\mathrm{m}^{2 \circ} \mathrm{C}\right. \\
)\end{array}$ & $\mathrm{Bi}$ & $\begin{array}{c}\mathrm{T}_{\mathrm{a}} \\
\left({ }^{\circ} \mathrm{C}\right)\end{array}$ & $\begin{array}{c}\mathrm{T}_{\mathrm{i}} \\
\left({ }^{\circ} \mathrm{C}\right)\end{array}$ & $\begin{array}{c}\mathrm{T}_{\mathrm{c}} \\
\left({ }^{\circ} \mathrm{C}\right)\end{array}$ & $\begin{array}{c}\text { tcexp } \\
\text { (min) }\end{array}$ & $\begin{array}{c}\text { tcpre } \\
\text { (min) }\end{array}$ & $\begin{array}{c}\mathrm{e} \\
(\%)\end{array}$ \\
\hline 1.35 & 107.0 & 3.21 & -27.9 & 30.6 & -18.0 & 48.5 & 41.1 & -15.18 \\
\hline 2.25 & 106.0 & 5.30 & -25.0 & 17.6 & -18.0 & 85.5 & 92.3 & 7.93 \\
\hline 0.75 & 104.0 & 1.73 & -20.1 & 5.0 & -18.0 & 21.8 & 24.0 & 10.00 \\
\hline 2.40 & 69.0 & 3.68 & -30.9 & 3.9 & -18.0 & 79.0 & 89.3 & 13.03 \\
\hline 0.60 & 68.0 & 0.91 & -25.4 & 30.2 & -18.0 & 30.4 & 23.4 & -23.03 \\
\hline 1.55 & 67.0 & 2.31 & -20.3 & 17.8 & -18.0 & 91.3 & 91.7 & 0.43 \\
\hline 0.55 & 8.5 & 0.10 & -30.1 & 17.3 & -18.0 & 124.5 & 117.9 & -5.29 \\
\hline 1.55 & 8.5 & 0.29 & -25.0 & 4.3 & -18.0 & 317.0 & 375.0 & 18.29 \\
\hline 2.25 & 10.5 & 0.53 & -19.0 & 30.3 & -18.0 & 885.0 & 739.7 & -16.42 \\
\hline
\end{tabular}

Cap. 2-50 
Tabla 2.15. Tiempos de congelación, experimentales y calculados. Placa plana, hígado de cerdo (Creed \& James, 1985).

\begin{tabular}{|c|c|c|c|c|c|c|c|c|}
\hline $\begin{array}{c}\mathrm{L} \\
(\mathrm{cm})\end{array}$ & $\begin{array}{c}\mathrm{h} \\
\mathrm{W} /\left(\mathrm{m}^{2 \circ} \mathrm{C}\right. \\
)\end{array}$ & $\mathrm{Bi}$ & $\begin{array}{c}\mathrm{T}_{\mathrm{a}} \\
\left({ }^{\circ} \mathrm{C}\right)\end{array}$ & $\begin{array}{c}\mathrm{T}_{\mathrm{i}} \\
\left({ }^{\circ} \mathrm{C}\right)\end{array}$ & $\begin{array}{c}\mathrm{T}_{\mathrm{c}} \\
\left({ }^{\circ} \mathrm{C}\right)\end{array}$ & $\begin{array}{c}t_{\text {cexp }} \\
(\mathrm{min})\end{array}$ & $\begin{array}{c}t_{\text {cpre }} \\
(\mathrm{min})\end{array}$ & $\begin{array}{c}\mathrm{e} \\
(\%)\end{array}$ \\
\hline 3.80 & 361.0 & 31.18 & -21.0 & 10.5 & -7.0 & 154.2 & 159.9 & 3.70 \\
\hline 3.80 & 361.0 & 31.18 & -23.2 & 9.0 & -7.0 & 139.2 & 141.1 & 1.37 \\
\hline 3.80 & 361.0 & 31.18 & -23.6 & 22.0 & -7.0 & 155.4 & 150.0 & -3.50 \\
\hline 3.80 & 361.0 & 31.18 & -24.6 & 8.6 & -7.0 & 121.8 & 131.6 & 8.08 \\
\hline 3.80 & 361.0 & 31.18 & -27.0 & 11.5 & -7.0 & 130.2 & 121.7 & -6.50 \\
\hline 3.80 & 361.0 & 31.18 & -26.7 & 9.5 & -7.0 & 129.6 & 121.2 & -6.47 \\
\hline 3.80 & 361.0 & 31.18 & -28.9 & 8.7 & -7.0 & 113.4 & 110.2 & -2.85 \\
\hline 3.80 & 361.0 & 31.18 & -29.0 & 14.3 & -7.0 & 110.4 & 114.7 & 3.86 \\
\hline 3.80 & 361.0 & 31.18 & -31.0 & 16.9 & -7.0 & 112.2 & 108.1 & -3.64 \\
\hline 3.80 & 361.0 & 31.18 & -32.5 & 15.8 & -7.0 & 114.6 & 102.0 & -11.00 \\
\hline 3.80 & 361.0 & 31.18 & -32.8 & 16.9 & -7.0 & 108.0 & 101.6 & -5.94 \\
\hline 3.80 & 361.0 & 31.18 & -34.3 & 4.0 & -7.0 & 86.4 & 85.5 & -0.98 \\
\hline 3.80 & 361.0 & 31.18 & -37.1 & 11.0 & -7.0 & 84.6 & 85.3 & 0.89 \\
\hline 3.80 & 361.0 & 31.18 & -38.5 & 21.2 & -7.0 & 88.2 & 86.9 & -1.44 \\
\hline 3.80 & 361.0 & 31.18 & -40.6 & 10.4 & -7.0 & 64.2 & 76.9 & 19.82 \\
\hline 5.10 & 361.0 & 41.84 & -21.0 & 10.0 & -7.0 & 268.8 & 275.9 & 2.66 \\
\hline 5.10 & 361.0 & 41.84 & -23.2 & 7.5 & -7.0 & 199.8 & 240.8 & 20.50 \\
\hline 5.10 & 361.0 & 41.84 & -23.6 & 23.4 & -7.0 & 278.4 & 261.4 & -6.12 \\
\hline 5.10 & 361.0 & 41.84 & -24.6 & 9.6 & -7.0 & 236.4 & 230.3 & -2.56 \\
\hline 5.10 & 361.0 & 41.84 & -27.0 & 11.1 & -7.0 & 211.2 & 210.3 & -0.42 \\
\hline 5.10 & 361.0 & 41.84 & -26.7 & 8.3 & -7.0 & 221.4 & 207.6 & -6.22 \\
\hline 5.10 & 361.0 & 41.84 & -29.0 & 7.7 & -7.0 & 197.4 & 188.2 & -4.65 \\
\hline 5.10 & 361.0 & 41.84 & -29.0 & 14.5 & -7.0 & 221.4 & 199.0 & -10.14 \\
\hline 5.10 & 361.0 & 41.84 & -31.0 & 14.5 & -7.0 & 181.2 & 184.8 & 1.99 \\
\hline 5.10 & 361.0 & 41.84 & -32.5 & 14.5 & -7.0 & 184.2 & 175.4 & -4.78 \\
\hline 5.10 & 361.0 & 41.84 & -32.8 & 16.0 & -7.0 & 204.0 & 175.2 & -14.13 \\
\hline 5.10 & 361.0 & 41.84 & -34.3 & 5.0 & -7.0 & 151.2 & 150.9 & -0.21 \\
\hline 5.10 & 361.0 & 41.84 & -37.1 & 9.3 & -7.0 & 162.0 & 145.8 & -10.02 \\
\hline 5.10 & 361.0 & 41.84 & -38.5 & 14.5 & -7.0 & 149.4 & 145.5 & -2.58 \\
\hline 5.10 & 361.0 & 41.84 & -40.6 & 10.2 & -7.0 & 133.2 & 133.1 & -0.08 \\
\hline 7.60 & 361.0 & 62.35 & -20.9 & 9.3 & -7.0 & 638.4 & 589.1 & -7.73 \\
\hline 7.60 & 361.0 & 62.35 & -23.2 & 8.6 & -7.0 & 592.8 & 520.5 & -12.20 \\
\hline 7.60 & 361.0 & 62.35 & -23.6 & 23.5 & -7.0 & 669.0 & 558.7 & -16.49 \\
\hline 7.60 & 361.0 & 62.35 & -24.6 & 11.0 & -7.0 & 544.2 & 498.1 & -8.48 \\
\hline 7.60 & 361.0 & 62.35 & -26.7 & 6.6 & -7.0 & 500.4 & 435.1 & -13.04 \\
\hline 7.60 & 361.0 & 62.35 & -29.0 & 9.3 & -7.0 & 466.2 & 408.8 & -12.32 \\
\hline 7.60 & 361.0 & 62.35 & -29.3 & 14.4 & -7.0 & 475.2 & 420.0 & -11.61 \\
\hline 7.60 & 361.0 & 62.35 & -30.5 & 9.0 & -7.0 & 458.4 & 385.5 & -15.91 \\
\hline 7.60 & 361.0 & 62.35 & -32.5 & 14.0 & -7.0 & 442.2 & 373.6 & -15.51 \\
\hline 7.60 & 361.0 & 62.35 & -32.8 & 16.8 & -7.0 & 449.4 & 376.0 & -16.34 \\
\hline 7.60 & 361.0 & 62.35 & -34.3 & 5.8 & $-7.0 \|$ & 387.6 & 326.3 & -15.82 \\
\hline 7.60 & 361.0 & 62.35 & -37.1 & 10.4 & -7.0 & 359.4 & 314.5 & -12.49 \\
\hline 7.60 & 361.0 & 62.35 & -38.5 & 15.2 & -7.0 & 361.8 & 312.3 & -13.68 \\
\hline 7.60 & 361.0 & 62.35 & -40.7 & 9.9 & -7.0 & 318.6 & 282.9 & -11.22 \\
\hline
\end{tabular}

Cap. 2-51 
Tabla 2.16. Tiempos de congelación, experimentales y calculados. Placa plana, puré de papas (Cleland \& Earle, 1977).

\begin{tabular}{|c|c|c|c|c|c|c|c|c|}
\hline $\begin{array}{c}\mathrm{L} \\
(\mathrm{cm})\end{array}$ & $\begin{array}{c}\mathrm{h} \\
\mathrm{W} /\left(\mathrm{m}^{2 \circ} \mathrm{C}\right. \\
)\end{array}$ & $\mathrm{Bi}$ & $\begin{array}{c}\mathrm{T}_{\mathrm{a}} \\
\left({ }^{\circ} \mathrm{C}\right)\end{array}$ & $\begin{array}{c}\mathrm{T}_{\mathrm{i}} \\
\left({ }^{\circ} \mathrm{C}\right)\end{array}$ & $\begin{array}{c}\mathrm{T}_{\mathrm{c}} \\
\left({ }^{\circ} \mathrm{C}\right)\end{array}$ & $\begin{array}{c}t_{\text {cexp }} \\
(\mathrm{min})\end{array}$ & $\begin{array}{c}\mathrm{t}_{\text {cpre }} \\
\text { (min) }\end{array}$ & $\begin{array}{c}\mathrm{e} \\
(\%)\end{array}$ \\
\hline 3.60 & 90.0 & 6.11 & -24.3 & 28.0 & -10.0 & 187.0 & 192.6 & $3.01 \|$ \\
\hline 3.60 & 51.9 & 3.53 & -24.9 & 11.8 & -10.0 & 223.0 & 233.2 & 4.56 \\
\hline 2.43 & 90.0 & 4.12 & -26.7 & 18.3 & -10.0 & 95.0 & 92.9 & -2.17 \\
\hline 2.43 & 21.6 & 0.99 & -25.0 & 15.0 & -10.0 & 269.0 & 275.2 & 2.29 \\
\hline 1.25 & 30.6 & 0.72 & -25.9 & 28.4 & -10.0 & 106.0 & 97.8 & -7.73 \\
\hline 1.25 & 13.6 & 0.32 & -25.9 & 4.7 & -10.0 & 181.0 & 175.8 & -2.90 \\
\hline
\end{tabular}

Tabla 2.17. Tiempos de congelación, experimentales y calculados. Placa plana, puré de papas (Hung \& Thompson, 1983).

\begin{tabular}{|c|c|c|c|c|c|c|c|c|}
\hline $\begin{array}{c}\mathrm{L} \\
(\mathrm{cm})\end{array}$ & $\begin{array}{c}\mathrm{h} \\
\mathrm{W} /\left(\mathrm{m}^{2^{\circ}} \mathrm{C}\right. \\
)\end{array}$ & $\mathrm{Bi}$ & $\begin{array}{c}\mathrm{T}_{\mathrm{a}} \\
\left({ }^{\circ} \mathrm{C}\right)\end{array}$ & $\begin{array}{c}\mathrm{T}_{\mathrm{i}} \\
\left({ }^{\circ} \mathrm{C}\right)\end{array}$ & $\begin{array}{c}\mathrm{T}_{\mathrm{c}} \\
\left({ }^{\circ} \mathrm{C}\right)\end{array}$ & $\begin{array}{c}t_{\text {cexp }} \\
\text { (min) }\end{array}$ & $\begin{array}{c}\mathrm{t}_{\text {cpre }} \\
\text { (min) }\end{array}$ & $\begin{array}{c}\mathrm{e} \\
(\%)\end{array}$ \\
\hline 1.40 & 108.0 & 2.85 & -30.9 & 30.4 & -18.0 & 44.0 & 39.1 & -11.21 \\
\hline 2.20 & 106.0 & 4.40 & -25.5 & 16.8 & -18.0 & 88.0 & 87.0 & -1.15 \\
\hline 0.50 & 104.0 & 0.98 & -20.0 & 4.6 & -18.0 & 17.6 & 15.4 & -12.26 \\
\hline 2.30 & 69.0 & 2.99 & -30.6 & 4.0 & -18.0 & 89.8 & 86.6 & -3.58 \\
\hline 0.50 & 68.0 & 0.64 & -26.0 & 29.2 & -18.0 & 23.0 & 19.6 & -14.77 \\
\hline 1.40 & 67.0 & 1.77 & -20.6 & 17.5 & -18.0 & 88.5 & 81.8 & -7.61 \\
\hline 0.50 & 8.5 & 0.08 & -29.6 & 17.8 & -18.0 & 130.8 & 117.8 & -9.96 \\
\hline 1.60 & 10.5 & 0.32 & -25.4 & 4.0 & -18.0 & 356.3 & 332.1 & -6.79 \\
\hline 2.30 & 10.5 & 0.46 & -20.5 & 30.2 & -18.0 & 811.0 & 740.9 & -8.65 \\
\hline
\end{tabular}

Tabla 2.18. Tiempos de congelación, experimentales y calculados. Placa plana, carpa (Hung \& Thompson, 1983).

\begin{tabular}{|c|c|c|c|c|c|c|c|c|}
\hline $\begin{array}{c}\mathrm{L} \\
(\mathrm{cm})\end{array}$ & $\begin{array}{c}\mathrm{h} \\
\mathrm{W} /\left(\mathrm{m}^{2 \circ} \mathrm{C}\right. \\
)\end{array}$ & $\mathrm{Bi}$ & $\begin{array}{c}\mathrm{T}_{\mathrm{a}} \\
\left({ }^{\circ} \mathrm{C}\right)\end{array}$ & $\begin{array}{c}\mathrm{T}_{\mathrm{i}} \\
\left({ }^{\circ} \mathrm{C}\right)\end{array}$ & $\begin{array}{c}\mathrm{T}_{\mathrm{c}} \\
\left({ }^{\circ} \mathrm{C}\right)\end{array}$ & $\begin{array}{c}t_{\text {cexp }} \\
(\mathrm{min})\end{array}$ & $\begin{array}{c}\mathrm{t}_{\text {cpre }} \\
\text { (min) }\end{array}$ & $\begin{array}{c}\mathrm{e} \\
(\%)\end{array}$ \\
\hline 1.45 & 108.0 & 3.26 & -30.2 & 31.3 & -18.0 & 49.0 & 42.8 & -12.66 \\
\hline 2.30 & 105.5 & 5.06 & -23.9 & 18.2 & -18.0 & 103.0 & 103.4 & 0.41 \\
\hline 0.60 & 104.0 & 1.30 & -20.2 & 5.3 & -18.0 & 18.2 & 19.1 & 4.75 \\
\hline 2.40 & 68.5 & 3.43 & -28.7 & 3.9 & -18.0 & 99.0 & 100.4 & 1.46 \\
\hline 0.50 & 68.0 & 0.71 & -25.1 & 29.9 & -18.0 & 27.0 & 20.2 & -25.02 \\
\hline 1.50 & 67.0 & 2.09 & -20.1 & 18.1 & -18.0 & 96.5 & 92.5 & -4.14 \\
\hline 0.50 & 8.5 & 0.09 & -29.6 & 17.4 & -18.0 & 124.8 & 115.4 & -7.57 \\
\hline 1.50 & 8.5 & 0.27 & -25.0 & 6.1 & -18.0 & 362.0 & 393.2 & 8.63 \\
\hline
\end{tabular}

Cap. 2-52 
Tiempos de congelación

\begin{tabular}{||cccccc|cc|c||}
\hline \hline $\begin{array}{c}\mathrm{L} \\
(\mathrm{cm})\end{array}$ & $\begin{array}{c}\mathrm{h} \\
\mathrm{W} /\left(\mathrm{m}^{2} \mathrm{C}\right. \\
)\end{array}$ & $\mathrm{Bi}$ & $\begin{array}{c}\mathrm{T}_{\mathrm{a}} \\
\left({ }^{\circ} \mathrm{C}\right)\end{array}$ & $\begin{array}{c}\mathrm{T}_{\mathrm{i}} \\
\left({ }^{\circ} \mathrm{C}\right)\end{array}$ & $\begin{array}{c}\mathrm{T}_{\mathrm{c}} \\
\left({ }^{\circ} \mathrm{C}\right)\end{array}$ & $\begin{array}{c}\mathrm{t}_{\text {cexp }} \\
(\mathrm{min})\end{array}$ & $\begin{array}{c}\mathrm{t}_{\text {cpre }} \\
(\mathrm{min})\end{array}$ & $\begin{array}{c}\mathrm{e} \\
(\%)\end{array}$ \\
\hline \hline 2.35 & 10.5 & 0.51 & -20.0 & 10.5 & -18.0 & 869.0 & 699.9 & -19.46 \\
\hline \hline
\end{tabular}

Cap. 2-53 
Tabla 2.19. Tiempos de congelación, experimentales y calculados. Placa plana, tiburón (Hense \& Kieckbusch, 1991).

\begin{tabular}{|c|c|c|c|c|c|c|c|c|}
\hline $\begin{array}{c}\mathrm{L} \\
(\mathrm{cm})\end{array}$ & $\begin{array}{c}\mathrm{h} \\
\mathrm{W} /\left(\mathrm{m}^{2 \circ} \mathrm{C}\right. \\
) \\
\end{array}$ & $\mathrm{Bi}$ & $\begin{array}{c}\mathrm{T}_{\mathrm{a}} \\
\left({ }^{\circ} \mathrm{C}\right)\end{array}$ & $\begin{array}{c}\mathrm{T}_{\mathrm{i}} \\
\left({ }^{\circ} \mathrm{C}\right)\end{array}$ & $\begin{array}{c}\mathrm{T}_{\mathrm{c}} \\
\left({ }^{\circ} \mathrm{C}\right)\end{array}$ & $\begin{array}{c}t_{\operatorname{cexp}} \\
(\mathrm{min})\end{array}$ & $\begin{array}{c}\mathrm{t}_{\text {cpre }} \\
(\mathrm{min})\end{array}$ & $\begin{array}{c}\mathrm{e} \\
(\%)\end{array}$ \\
\hline 2.00 & 156.0 & 6.89 & -25.0 & 7.0 & -10.0 & 56.6 & 58.7 & 3.82 \\
\hline 2.00 & 156.0 & 6.89 & -35.0 & 7.0 & -10.0 & 37.5 & 40.5 & 7.91 \\
\hline 2.20 & 156.0 & 7.58 & -45.0 & 7.0 & -10.0 & 37.2 & 35.7 & -4.12 \\
\hline 3.00 & 156.0 & 10.33 & -25.0 & 7.0 & -10.0 & 106.4 & 112.7 & 5.95 \\
\hline 2.80 & 156.0 & 9.64 & -35.0 & 7.0 & -10.0 & 62.3 & 69.3 & 11.26 \\
\hline 3.10 & 156.0 & 10.68 & -45.0 & 7.0 & -10.0 & 57.9 & 62.2 & 7.48 \\
\hline
\end{tabular}

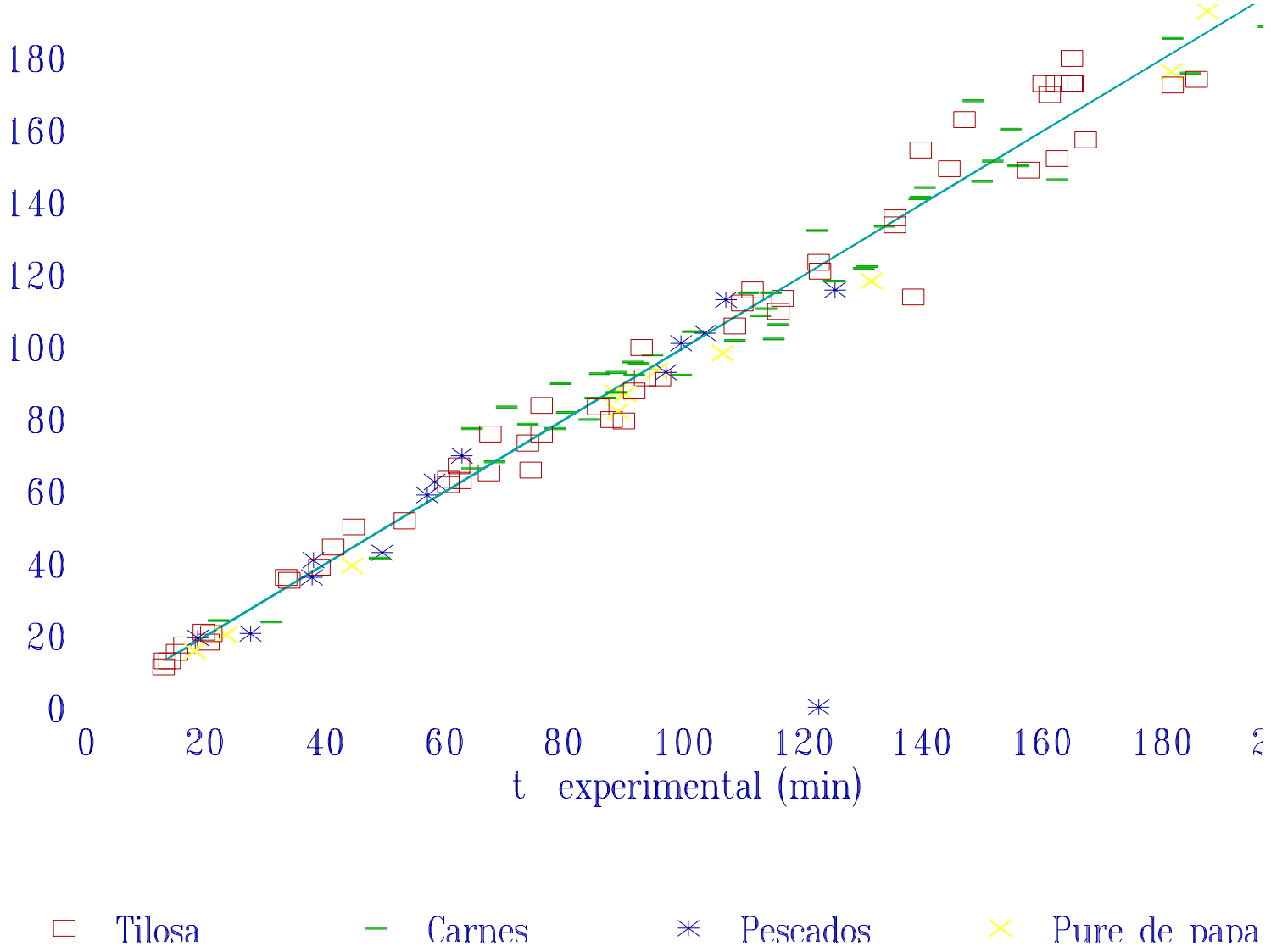

Figura 2.11a. Tiempos de congelación calculados vs. experimentales, placa plana (perpendicular a las fibras); diferenciados por tipo de producto.

Cap. 2-54 


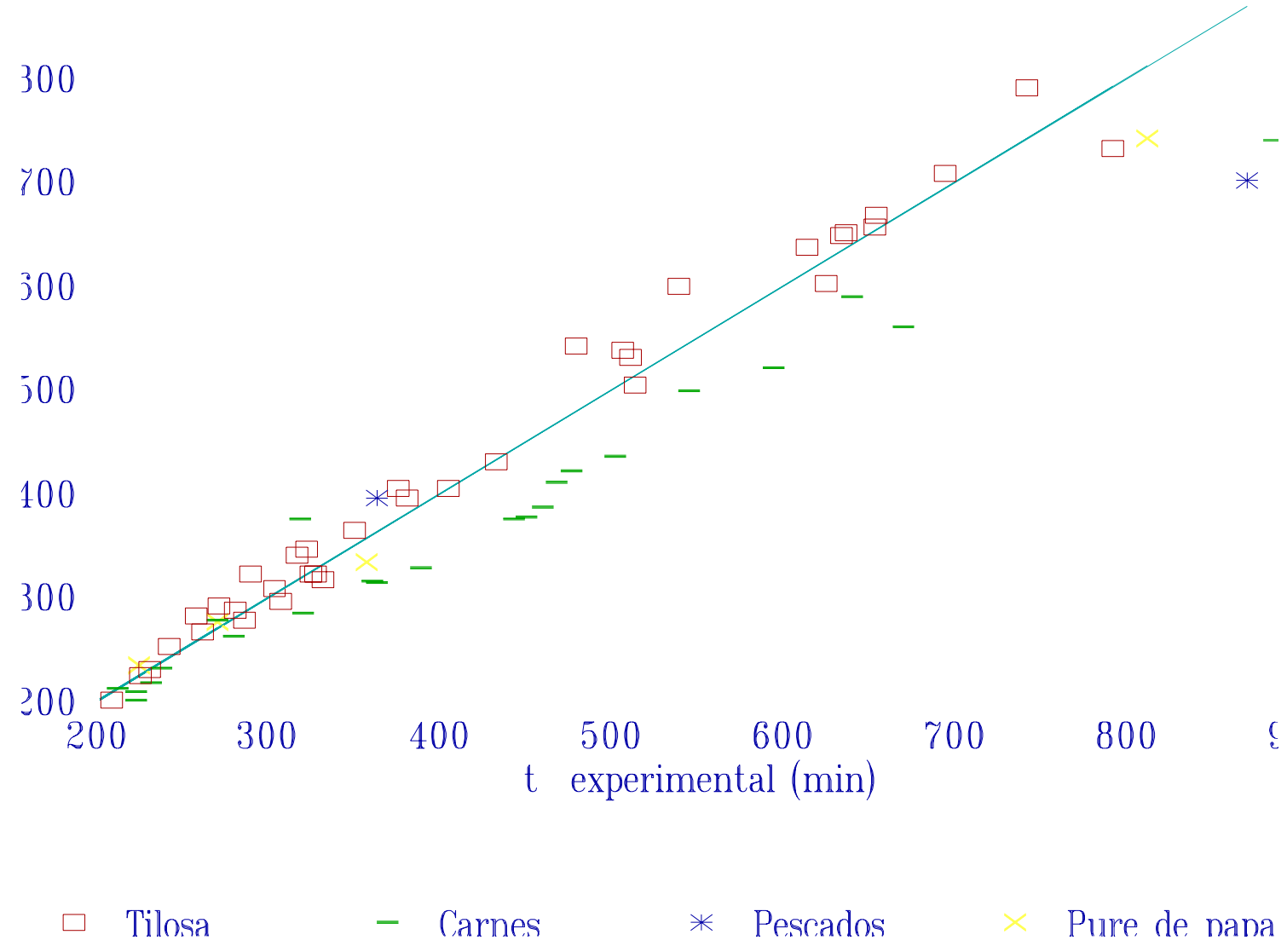

Figura 2.11b. Tiempos de congelación calculados vs. experimentales, placa plana (perpendicular a las fibras); diferenciados por tipo de producto. 


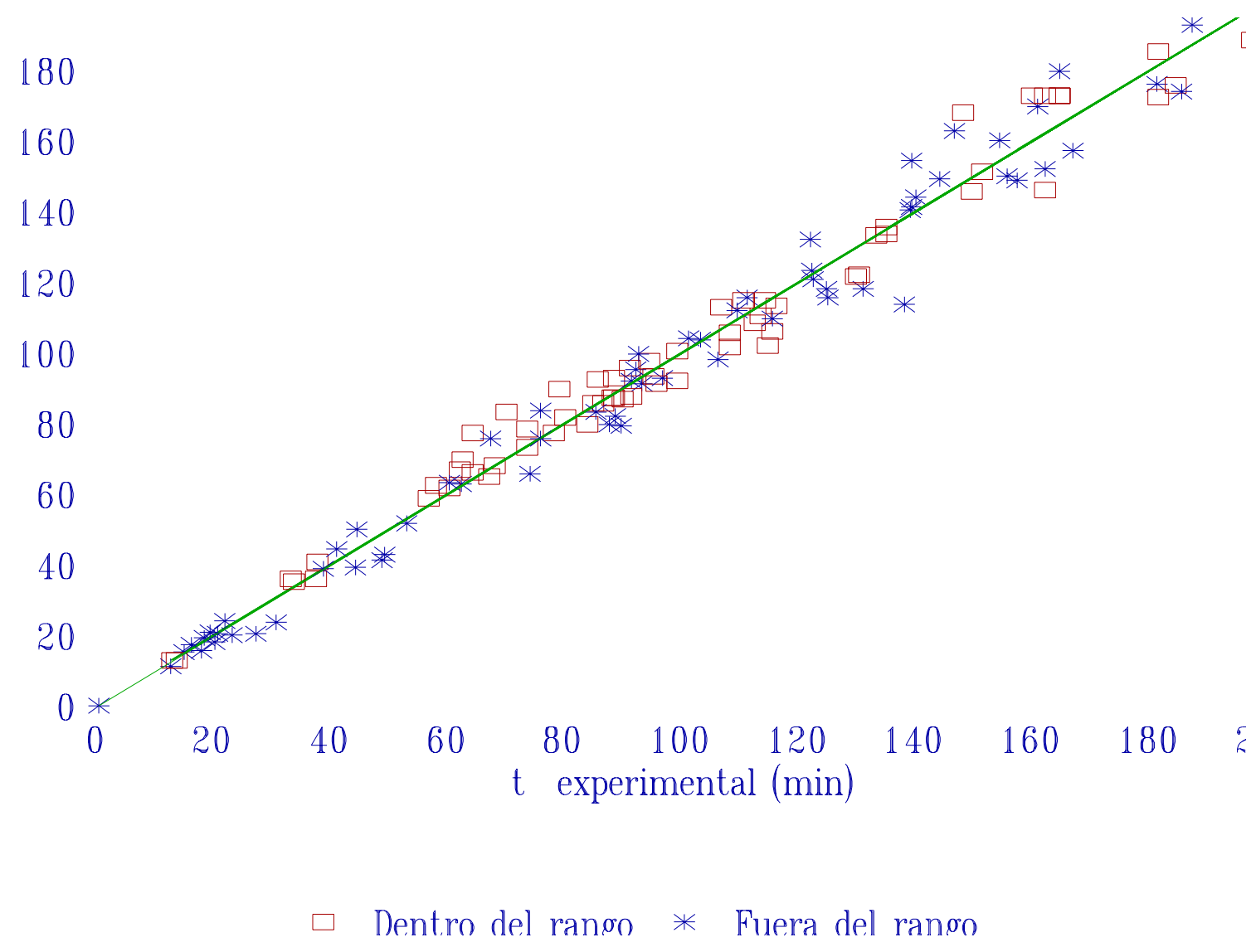

Figura 2.12a. Tiempos de congelación calculados vs. experimentales, placa plana (perpendicular a las fibras); diferenciados según sus condiciones operativas estén dentro o fuera del rango de estricta validez del método de predicción. 


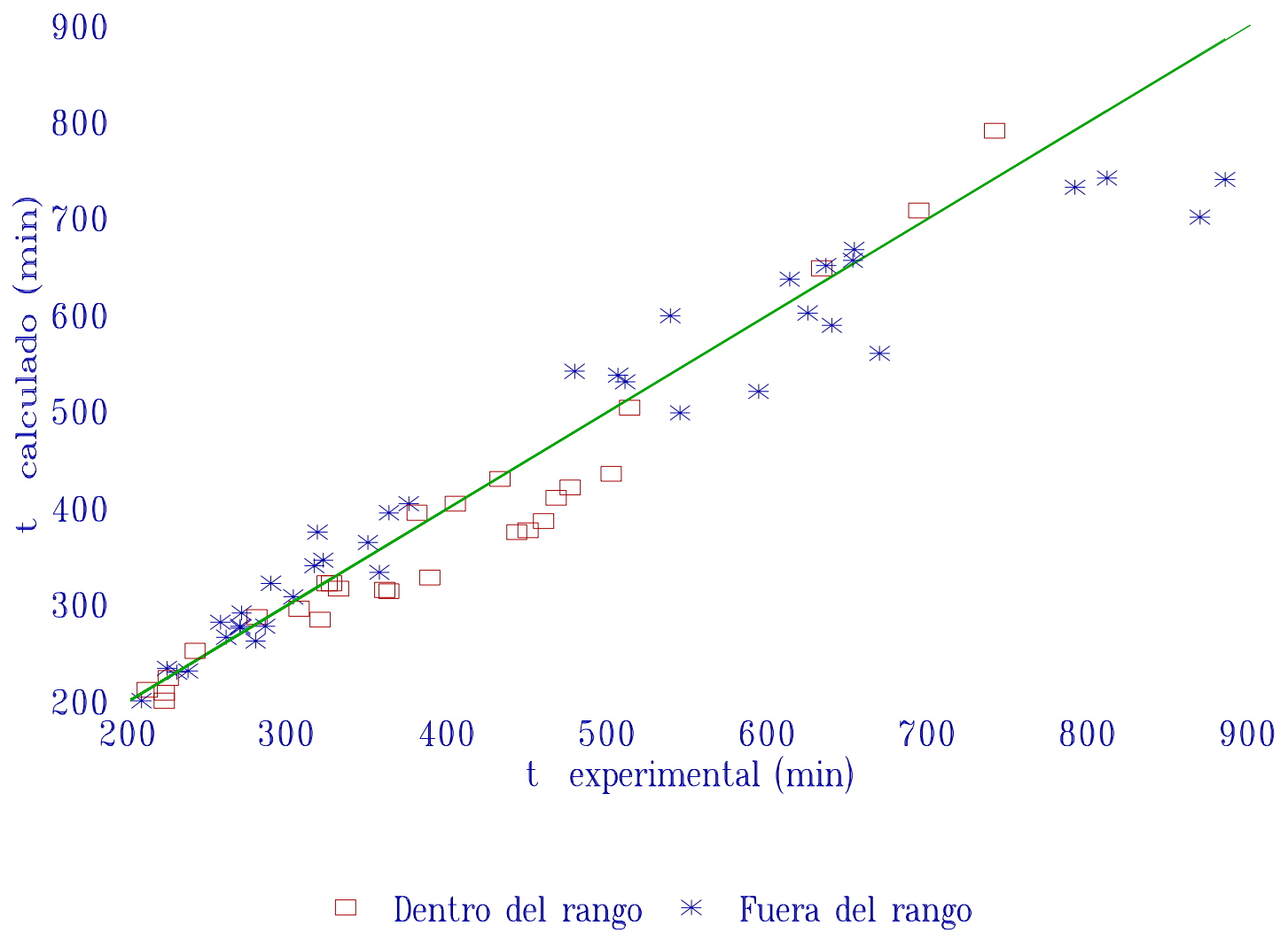

Figura 2.12b. Tiempos de congelación calculados vs. experimentales, placa plana (perpendicular a las fibras); diferenciados según sus condiciones operativas estén dentro o fuera del rango de estricta validez del método de predicción. 
Tabla 2.20. Tiempos de congelación, experimentales y calculados. Placa plana (con transferencia de calor paralela a las fibras), carne vacuna (Mascheroni \& Calvelo, 1982).

\begin{tabular}{|c|c|c|c|c|c|c|c|c|}
\hline $\begin{array}{c}\mathrm{L} \\
(\mathrm{cm})\end{array}$ & $\begin{array}{c}\mathrm{h} \\
\mathrm{W} /\left(\mathrm{m}^{2 \circ} \mathrm{C}\right. \\
)\end{array}$ & $\mathrm{Bi}$ & $\begin{array}{c}\mathrm{T}_{\mathrm{a}} \\
\left({ }^{\circ} \mathrm{C}\right)\end{array}$ & $\begin{array}{c}\mathrm{T}_{\mathrm{i}} \\
\left({ }^{\circ} \mathrm{C}\right)\end{array}$ & $\begin{array}{c}\mathrm{T}_{\mathrm{c}} \\
\left({ }^{\circ} \mathrm{C}\right)\end{array}$ & $\begin{array}{c}t_{\text {cexp }} \\
\text { (min) }\end{array}$ & $\begin{array}{c}\mathrm{t}_{\text {cpre }} \\
\text { (min) }\end{array}$ & $\begin{array}{c}\mathrm{e} \\
(\%)\end{array}$ \\
\hline 3.00 & 96.9 & 5.70 & -43.9 & 19.4 & -18.0 & 64.5 & 80.1 & 24.23 \\
\hline 4.70 & 22.8 & 2.10 & -42.0 & 16.0 & -18.0 & 330.0 & 383.0 & 16.07 \\
\hline 4.70 & 120.5 & 11.10 & -36.5 & 8.0 & -18.0 & 170.0 & 164.5 & -3.23 \\
\hline 4.00 & 120.5 & 9.45 & -33.0 & 21.0 & -18.0 & 171.0 & 154.7 & -9.54 \\
\hline
\end{tabular}

Tabla 2.21. Tiempos de congelación, experimentales y calculados. Placa plana (con transferencia de calor paralela a las fibras), carne vacuna (Hung \& Thompson, 1983).

\begin{tabular}{|c|c|c|c|c|c|c|c|c|}
\hline $\begin{array}{c}\mathrm{L} \\
(\mathrm{cm})\end{array}$ & $\begin{array}{c}\mathrm{h} \\
\mathrm{W} /\left(\mathrm{m}^{2 \circ} \mathrm{C}\right. \\
) \\
\end{array}$ & $\mathrm{Bi}$ & $\begin{array}{c}\mathrm{T}_{\mathrm{a}} \\
\left({ }^{\circ} \mathrm{C}\right)\end{array}$ & $\begin{array}{c}\mathrm{T}_{\mathrm{i}} \\
\left({ }^{\circ} \mathrm{C}\right)\end{array}$ & $\begin{array}{c}\mathrm{T}_{\mathrm{c}} \\
\left({ }^{\circ} \mathrm{C}\right)\end{array}$ & $\begin{array}{c}\text { tcexp } \\
\text { (min) }\end{array}$ & $\begin{array}{c}t_{\text {cpre }} \\
(\mathrm{min})\end{array}$ & $\begin{array}{c}\mathrm{e} \\
(\%)\end{array}$ \\
\hline 1.50 & 107.0 & 3.15 & -28.0 & 30.8 & -18.0 & 58.5 & 48.3 & -17.40 \\
\hline 2.40 & 106.0 & 4.99 & -25.2 & 17.4 & -18.0 & 108.3 & 99.0 & -8.56 \\
\hline 0.95 & 104.0 & 1.94 & -20.3 & 4.8 & -18.0 & 32.2 & 32.6 & 1.36 \\
\hline 2.35 & 69.0 & 3.18 & -27.6 & 4.3 & -18.0 & 104.8 & 99.5 & -5.07 \\
\hline 0.70 & 68.0 & 0.93 & -25.8 & 29.8 & -18.0 & 34.8 & 29.5 & -15.33 \\
\hline 1.80 & 67.0 & 2.36 & -20.8 & 17.9 & -18.0 & 118.0 & 111.1 & -5.83 \\
\hline 0.70 & 8.5 & 0.12 & -30.3 & 17.2 & -18.0 & 163.5 & 166.1 & 1.58 \\
\hline 1.65 & 8.5 & 0.28 & -25.2 & 4.8 & -18.0 & 390.0 & 435.5 & 11.67 \\
\hline 2.35 & 10.5 & 0.48 & -20.7 & 30.8 & -18.0 & 846.0 & 765.5 & -9.51 \\
\hline
\end{tabular}

Cap. 2-58 


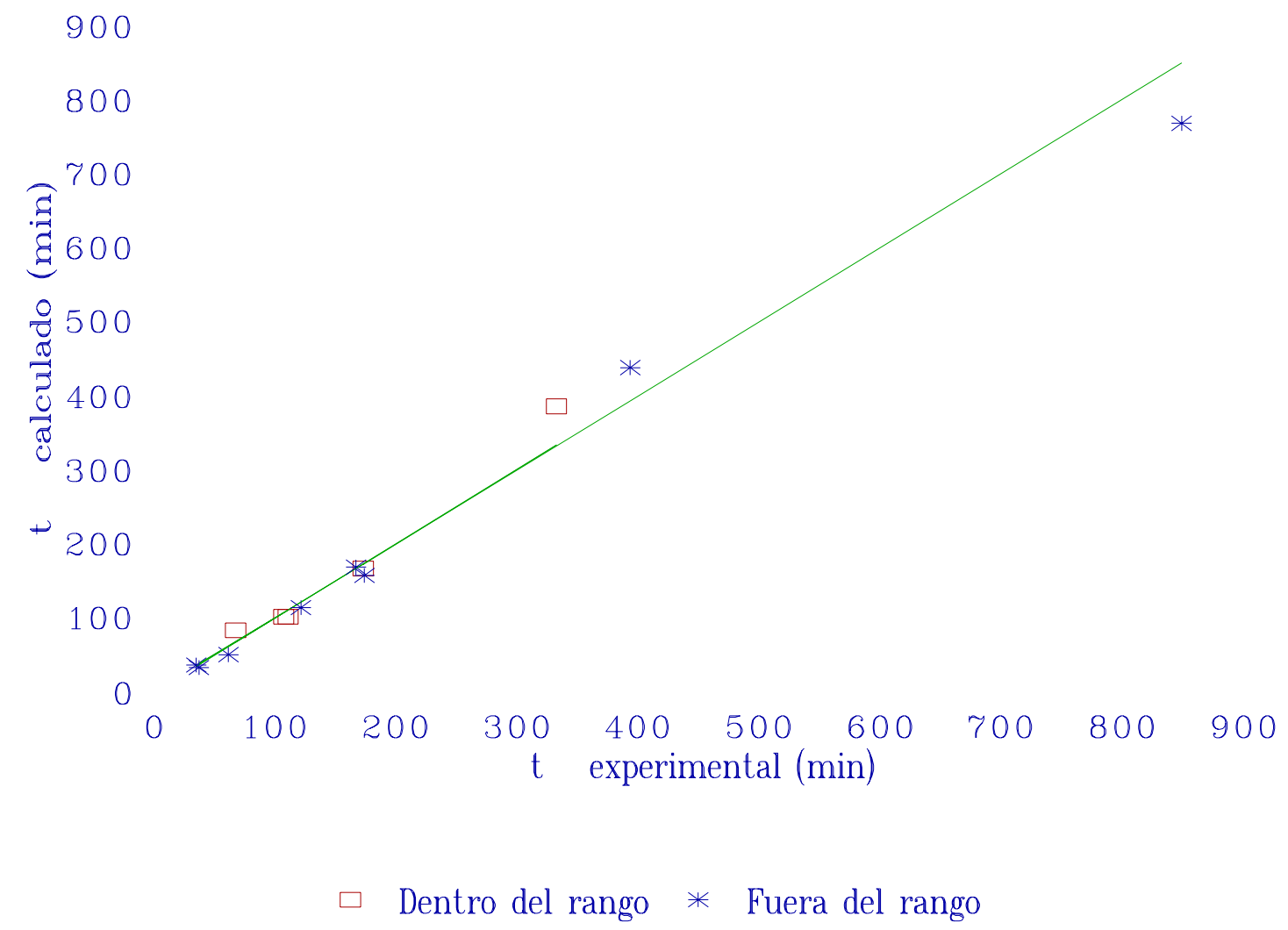

Figura 2.13. Tiempos de congelación calculados vs. experimentales, placa plana (carnes con transferencia de calor paralela a las fibras); diferenciados según sus condiciones operativas estén dentro o fuera del rango de estricta validez del método de predicción. 
Tabla 2.22. Tiempos de congelación, experimentales y calculados. Cilindro infinito, tilosa (Cleland \& Earle, 1979).

\begin{tabular}{|c|c|c|c|c|c|c|c|c|}
\hline $\begin{array}{c}\mathrm{L} \\
(\mathrm{cm})\end{array}$ & $\begin{array}{c}\mathrm{h} \\
\mathrm{W} /\left(\mathrm{m}^{2 \circ} \mathrm{C}\right. \\
)\end{array}$ & $\mathrm{Bi}$ & $\begin{array}{c}\mathrm{T}_{\mathrm{a}} \\
\left({ }^{\circ} \mathrm{C}\right)\end{array}$ & $\begin{array}{c}\mathrm{T}_{\mathrm{i}} \\
\left({ }^{\circ} \mathrm{C}\right)\end{array}$ & $\begin{array}{c}\mathrm{T}_{\mathrm{c}} \\
\left({ }^{\circ} \mathrm{C}\right)\end{array}$ & $\begin{array}{c}t_{\text {cexp }} \\
(\mathrm{min})\end{array}$ & $\begin{array}{c}t_{\text {cpre }} \\
(\mathrm{min})\end{array}$ & $\begin{array}{c}\mathrm{e} \\
(\%)\end{array}$ \\
\hline 7.68 & 36.4 & 4.58 & -21.0 & 28.4 & -10.0 & 501.6 & 502.8 & $0.24-16.89$ \\
\hline 7.68 & 36.4 & 4.58 & -33.5 & 27.2 & -10.0 & 366.0 & 304.2 & 0.14 \\
\hline 7.68 & 36.4 & 4.58 & -19.8 & 26.7 & -10.0 & 534.0 & 534.8 & -4.67 \\
\hline 7.68 & 36.4 & 4.58 & -19.8 & 27.6 & -10.0 & 561.6 & 535.4 & 5.31 \\
\hline 7.68 & 36.4 & 4.58 & -20.0 & 21.0 & -10.0 & 498.0 & 524.5 & 4.68 \\
\hline 7.68 & 36.4 & 4.58 & -21.1 & 4.8 & -10.0 & 450.0 & 471.1 & -10.76 \\
\hline 7.68 & 36.4 & 4.58 & -33.6 & 16.2 & -10.0 & 333.6 & 297.7 & -10.43 \\
\hline 7.68 & 36.4 & 4.58 & -33.5 & 10.8 & -10.0 & 328.8 & 294.5 & -3.83 \\
\hline 7.68 & 21.0 & 2.64 & -39.8 & 4.0 & -10.0 & 342.0 & 328.9 & -4.60 \\
\hline 7.68 & 21.0 & 2.64 & -40.5 & 13.0 & -10.0 & 351.6 & 335.4 & 4.45 \\
\hline 5.23 & 27.2 & 2.33 & -20.0 & 19.0 & -10.0 & 349.2 & 364.7 & 7.29 \\
\hline 5.23 & 27.2 & 2.33 & -20.5 & 5.8 & -10.0 & 318.0 & 341.2 & -4.20 \\
\hline 5.23 & 27.2 & 2.33 & -33.4 & 16.5 & -10.0 & 218.4 & 209.2 & -4.77 \\
\hline 5.23 & 27.2 & 2.33 & -34.0 & 4.4 & -10.0 & 206.4 & 196.6 & 2.32 \\
\hline 5.23 & 27.2 & 2.33 & -25.6 & 20.1 & -10.0 & 273.6 & 279.9 & -2.47 \\
\hline 5.23 & 27.2 & 2.33 & -25.8 & 19.6 & -10.0 & 284.4 & 277.4 & -1.98 \\
\hline 5.23 & 17.8 & 1.52 & -39.9 & 11.5 & -10.0 & 237.6 & 232.9 & -4.71 \\
\hline 5.23 & 17.8 & 1.52 & -39.6 & 14.6 & -10.0 & 248.4 & 236.7 & -0.99 \\
\hline 5.23 & 27.2 & 2.33 & -20.3 & 30.0 & -10.0 & 368.4 & 364.8 & -12.53 \\
\hline 5.23 & 27.2 & 2.33 & -33.1 & 27.8 & -10.0 & 246.0 & 215.2 & 4.21 \\
\hline 2.60 & 35.5 & 1.51 & -20.0 & 19.7 & -10.0 & 118.8 & 123.8 & 6.46 \\
\hline 2.60 & 35.5 & 1.51 & -20.2 & 5.8 & -10.0 & 110.4 & 117.5 & 0.97 \\
\hline 2.60 & 35.5 & 1.51 & -33.2 & 17.2 & -10.0 & 70.8 & 71.5 & 5.75 \\
\hline 2.60 & 35.5 & 1.51 & -33.2 & 4.8 & -10.0 & 64.8 & 68.5 & 6.36 \\
\hline 2.60 & 35.5 & 1.51 & -33.5 & 4.3 & -10.0 & 63.6 & 67.6 & 7.49 \\
\hline 2.60 & 35.5 & 1.51 & -33.7 & 4.0 & -10.0 & 62.4 & 67.1 & -0.72 \\
\hline 2.60 & 23.9 & 1.02 & -40.2 & 15.4 & -10.0 & 80.4 & 79.8 & -2.75 \\
\hline 2.60 & 35.5 & 1.51 & -19.9 & 28.0 & -10.0 & 129.6 & 126.0 & -6.05 \\
\hline 2.60 & 35.5 & 1.51 & -33.4 & 26.8 & -10.0 & 76.8 & 72.2 & -12.63 \\
\hline 2.60 & 23.9 & 1.02 & -30.2 & 25.3 & -10.0 & 126.0 & 110.1 & \\
\hline
\end{tabular}

Cap. 2-60 
Tabla 2.23. Tiempos de congelación, experimentales y calculados. Cilindro infinito, camarón (Wang \& Kolbe, 1987).

\begin{tabular}{|c|c|c|c|c|c|c|c|c|}
\hline $\begin{array}{c}\mathrm{L} \\
(\mathrm{cm})\end{array}$ & $\begin{array}{c}\mathrm{h} \\
\mathrm{W} /\left(\mathrm{m}^{2 \circ} \mathrm{C}\right. \\
) \\
\end{array}$ & $\mathrm{Bi}$ & $\begin{array}{c}\mathrm{T}_{\mathrm{a}} \\
\left({ }^{\circ} \mathrm{C}\right)\end{array}$ & $\begin{array}{c}\mathrm{T}_{\mathrm{i}} \\
\left({ }^{\circ} \mathrm{C}\right)\end{array}$ & $\begin{array}{c}\mathrm{T}_{\mathrm{c}} \\
\left({ }^{\circ} \mathrm{C}\right)\end{array}$ & $\begin{array}{c}t_{\text {cexp }} \\
\text { (min) }\end{array}$ & $\begin{array}{c}\mathrm{t}_{\text {cpre }} \\
\text { (min) }\end{array}$ & $\begin{array}{c}\mathrm{e} \\
(\%)\end{array}$ \\
\hline 7.60 & 12.6 & 2.48 & -21.7 & 15.6 & $-7.8 \|$ & 852.0 & 792.3 & -7.00 \\
\hline 7.60 & 12.6 & 2.48 & -21.7 & 15.6 & $-7.8 \|$ & 822.0 & 792.3 & -3.61 \\
\hline 7.60 & 15.7 & 3.09 & -23.6 & 15.6 & $-7.5 \|$ & 678.0 & 621.0 & -8.41 \\
\hline 7.60 & 15.7 & 3.09 & -23.6 & 11.7 & -7.2 & 666.0 & 611.2 & -8.23 \\
\hline 5.10 & 16.7 & 2.55 & -21.7 & 1.1 & $-8.3 \|$ & 324.0 & 326.9 & 0.91 \\
\hline 5.10 & 17.3 & 2.49 & -23.6 & 15.0 & -7.8 & 342.0 & 324.8 & -5.03 \\
\hline
\end{tabular}

300

500

100

300

300

100

0

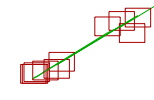

$\begin{array}{lllllllll}0 & 100 & 200 & 300 & 400 & 500 & 600 & 700 & 800\end{array}$

$t$ experimental (min)

Tilosa

Camaron

Figura 2.14. Tiempos de congelación calculados vs. experimentales, cilindro infinito; diferenciados por tipo de producto. 


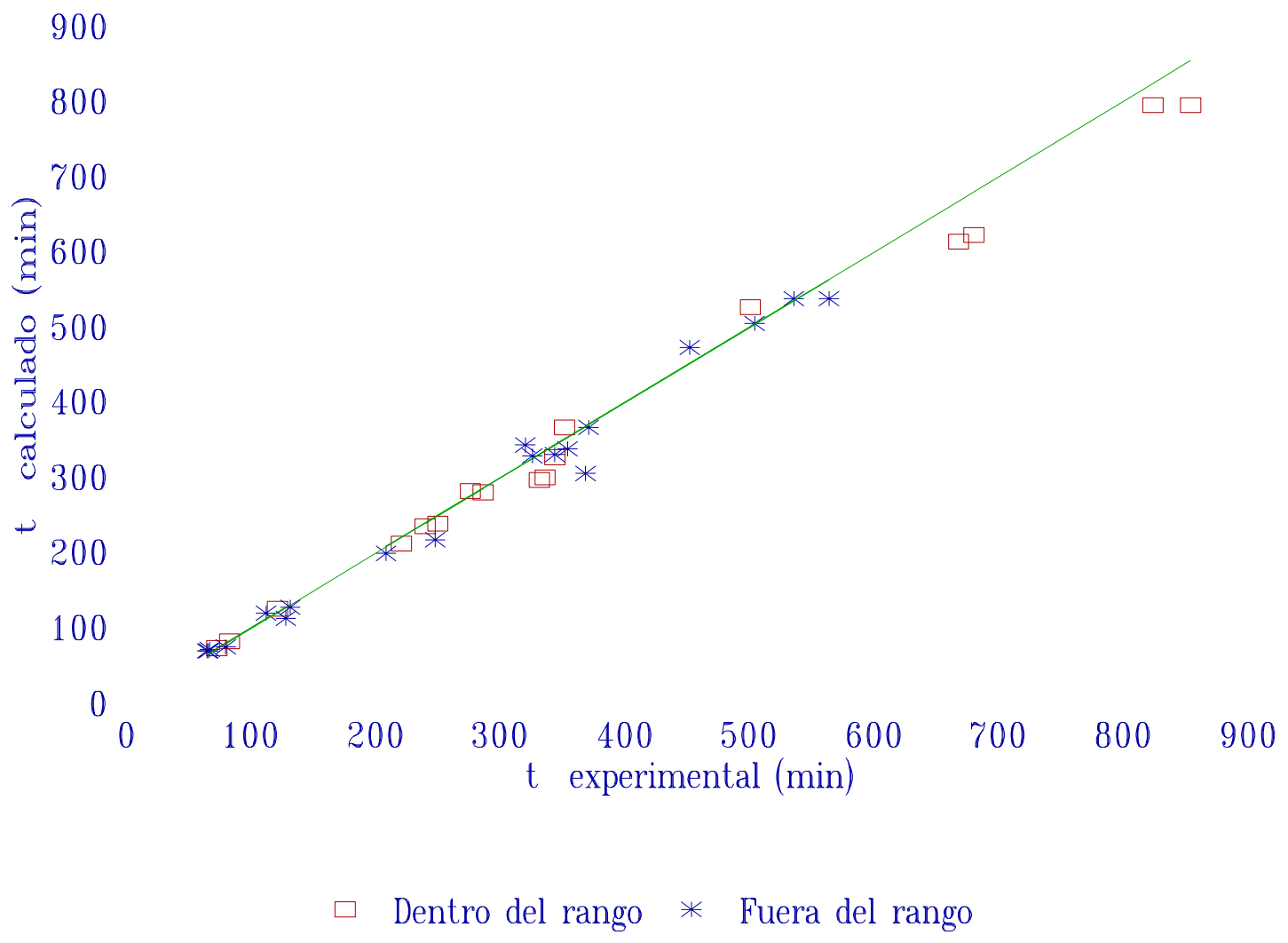

Figura 2.15. Tiempos de congelación calculados vs. experimentales, cilindro infinito; diferenciados según sus condiciones operativas estén dentro o fuera del rango de estricta validez del método de predicción. 
Tabla 2.24. Tiempos de congelación, experimentales y calculados. Esfera, tilosa (Cleland \& Earle, 1979).

\begin{tabular}{|c|c|c|c|c|c|c|c|c|}
\hline $\begin{array}{c}\mathrm{L} \\
(\mathrm{cm})\end{array}$ & $\begin{array}{c}\mathrm{h} \\
\mathrm{W} /\left(\mathrm{m}^{2 \circ} \mathrm{C}\right. \\
)\end{array}$ & $\mathrm{Bi}$ & $\begin{array}{c}\mathrm{T}_{\mathrm{a}} \\
\left({ }^{\circ} \mathrm{C}\right)\end{array}$ & $\begin{array}{c}\mathrm{T}_{\mathrm{i}} \\
\left({ }^{\circ} \mathrm{C}\right)\end{array}$ & $\begin{array}{c}\mathrm{T}_{\mathrm{c}} \\
\left({ }^{\circ} \mathrm{C}\right)\end{array}$ & $\begin{array}{c}t_{\text {cexp }} \\
(\mathrm{min})\end{array}$ & $\begin{array}{c}\mathrm{t}_{\text {cpre }} \\
(\mathrm{min})\end{array}$ & $\begin{array}{c}\mathrm{e} \\
(\%)\end{array}$ \\
\hline 7.88 & 45.9 & 6.57 & -33.2 & 16.0 & -10.0 & 181.2 & 187.0 & 3.22 \\
\hline 7.88 & 26.9 & 3.85 & -39.9 & 13.0 & -10.0 & 208.8 & 201.1 & -3.71 \\
\hline 7.88 & 26.9 & 3.85 & -39.7 & 4.6 & -10.0 & 198.0 & 189.1 & -4.50 \\
\hline 4.53 & 53.0 & 4.36 & -33.2 & 15.8 & -10.0 & 78.0 & 76.6 & -1.77 \\
\hline 4.53 & 53.0 & 4.36 & -33.5 & 11.4 & -10.0 & 73.2 & 74.2 & 1.36 \\
\hline 4.53 & 53.0 & 4.36 & -26.0 & 20.8 & -10.0 & 100.8 & 102.6 & 1.77 \\
\hline 4.53 & 53.0 & 4.36 & -26.0 & 22.1 & -10.0 & 100.8 & 103.0 & 2.20 \\
\hline 4.53 & 28.9 & 2.38 & -39.6 & 13.7 & -10.0 & 97.2 & 92.5 & -4.82 \\
\hline 4.53 & 28.9 & 2.38 & -29.3 & 4.0 & -10.0 & 116.4 & 119.5 & 2.66 \\
\hline 2.68 & 46.7 & 2.27 & -32.9 & 15.8 & -10.0 & 44.4 & 41.4 & -6.66 \\
\hline 2.68 & 46.7 & 2.27 & -33.4 & 6.1 & -10.0 & 40.8 & 38.3 & -6.20 \\
\hline 2.68 & 46.7 & 2.27 & -33.6 & 4.7 & -10.0 & 39.6 & 37.4 & -5.52 \\
\hline 2.68 & 46.7 & 2.27 & -33.7 & 4.6 & -10.0 & 39.6 & 37.2 & -5.96 \\
\hline 2.68 & 27.3 & 1.33 & -39.6 & 13.0 & -10.0 & 50.4 & 50.5 & 0.10 \\
\hline 7.88 & 45.9 & 6.57 & -20.9 & 28.5 & -10.0 & 310.8 & 327.1 & 5.25 \\
\hline 7.88 & 45.9 & 6.57 & -20.0 & 18.7 & -10.0 & 308.4 & 333.9 & 8.25 \\
\hline 7.88 & 45.9 & 6.57 & -20.8 & 4.9 & -10.0 & 270.0 & 292.4 & 8.31 \\
\hline 7.88 & 45.9 & 6.57 & -33.2 & 28.2 & -10.0 & 202.8 & 194.6 & -4.06 \\
\hline 7.88 & 45.9 & 6.57 & -32.6 & 1.5 & -10.0 & 162.0 & 165.9 & 2.43 \\
\hline 7.88 & 45.9 & 6.57 & -21.1 & 29.3 & -10.0 & 309.6 & 324.2 & 4.73 \\
\hline 7.88 & 45.9 & 6.57 & -20.8 & 29.6 & -10.0 & 314.4 & 329.8 & 4.89 \\
\hline 4.53 & 53.0 & 4.36 & -19.9 & 30.0 & -10.0 & 136.8 & 142.3 & 4.02 \\
\hline 4.53 & 53.0 & 4.36 & -20.0 & 18.7 & -10.0 & 127.2 & 136.9 & 7.61 \\
\hline 4.53 & 53.0 & 4.36 & -20.5 & 5.7 & -10.0 & 115.2 & 123.0 & 6.79 \\
\hline 4.53 & 53.0 & 4.36 & -33.6 & 30.4 & -10.0 & 84.0 & 79.1 & -5.79 \\
\hline 2.68 & 46.7 & 2.27 & -19.9 & 27.2 & -10.0 & 72.0 & 75.7 & 5.10 \\
\hline 2.68 & 46.7 & 2.27 & -20.0 & 23.8 & -10.0 & 68.4 & 74.5 & 8.97 \\
\hline 2.68 & 46.7 & 2.27 & -20.4 & 3.2 & -10.0 & 57.6 & 64.0 & 11.15 \\
\hline 2.68 & 46.7 & 2.27 & -33.4 & 29.6 & -10.0 & 48.0 & 42.6 & -11.30 \\
\hline 2.68 & 27.3 & 1.33 & -29.3 & 29.1 & -10.0 & 75.6 & 74.6 & -1.39 \\
\hline
\end{tabular}

Cap. 2-63 
Tabla 2.25. Tiempos de congelación, experimentales y calculados. Esfera, albóndigas de carne picada (Tocci \& Mascheroni, 1993).

\begin{tabular}{|c|c|c|c|c|c|c|c|c|}
\hline $\begin{array}{c}\mathrm{L} \\
(\mathrm{cm})\end{array}$ & $\begin{array}{c}\mathrm{h} \\
\mathrm{W} /\left(\mathrm{m}^{2 \circ} \mathrm{C}\right. \\
) \\
\end{array}$ & $\mathrm{Bi}$ & $\begin{array}{c}\mathrm{T}_{\mathrm{a}} \\
\left({ }^{\circ} \mathrm{C}\right)\end{array}$ & $\begin{array}{c}\mathrm{T}_{\mathrm{i}} \\
\left({ }^{\circ} \mathrm{C}\right)\end{array}$ & $\begin{array}{c}\mathrm{T}_{\mathrm{c}} \\
\left({ }^{\circ} \mathrm{C}\right)\end{array}$ & $\begin{array}{c}t_{\text {cexp }} \\
(\mathrm{min})\end{array}$ & $\begin{array}{c}\mathrm{t}_{\text {cpre }} \\
(\mathrm{min})\end{array}$ & $\begin{array}{c}\mathrm{e} \\
(\%)\end{array}$ \\
\hline 1.90 & 21.0 & 0.90 & -23.0 & 13.3 & -18.0 & 81.7 & 80.6 & -1.33 \\
\hline 1.90 & 21.0 & 0.90 & -22.9 & 2.8 & -18.0 & 75.8 & 73.5 & -2.98 \\
\hline 1.90 & 21.1 & 0.91 & -28.0 & -0.5 & -18.0 & 52.4 & 50.4 & -3.82 \\
\hline 1.90 & 21.1 & 0.91 & -28.6 & 2.1 & -18.0 & 59.6 & 56.2 & -5.65 \\
\hline 1.90 & 29.1 & 1.25 & -24.3 & 3.6 & -18.0 & 49.7 & 52.9 & 6.41 \\
\hline 1.90 & 29.1 & 1.25 & -25.0 & -0.1 & -18.0 & 43.5 & 45.5 & 4.54 \\
\hline 1.90 & 29.4 & 1.27 & -30.8 & 10.4 & -18.0 & 41.0 & 43.0 & 4.82 \\
\hline 1.90 & 29.4 & 1.27 & -30.5 & 19.0 & -18.0 & 44.8 & 45.3 & 1.05 \\
\hline 1.90 & 42.5 & 1.83 & -24.8 & 1.2 & -18.0 & 37.0 & 36.2 & -2.13 \\
\hline 1.90 & 42.5 & 1.83 & -24.9 & 10.3 & -18.0 & 38.6 & 40.6 & 5.23 \\
\hline 1.90 & 42.9 & 1.85 & -28.9 & 1.8 & -18.0 & 30.1 & 30.8 & 2.42 \\
\hline 1.90 & 42.9 & 1.85 & -30.8 & 13.1 & -18.0 & 31.0 & 32.3 & 4.23 \\
\hline 1.90 & 59.1 & 2.55 & -24.8 & 6.6 & -18.0 & 28.9 & 31.1 & 7.66 \\
\hline 1.90 & 59.1 & 2.55 & -25.0 & 7.6 & -18.0 & 29.4 & 31.1 & 5.83 \\
\hline 1.90 & 59.3 & 2.55 & -28.9 & 11.2 & -18.0 & 25.2 & 27.1 & 7.43 \\
\hline 1.90 & 59.3 & 2.55 & -28.9 & 1.4 & -18.0 & 22.7 & 24.0 & 5.91 \\
\hline 1.90 & 21.1 & 0.91 & -24.9 & 10.1 & -18.0 & 68.5 & 72.1 & 5.22 \\
\hline 1.90 & 21.1 & 0.91 & -23.7 & 1.3 & -18.0 & 66.3 & 67.9 & 2.45 \\
\hline 1.90 & 21.1 & 0.91 & -30.2 & 5.4 & -18.0 & 61.0 & 55.8 & -8.54 \\
\hline 1.90 & 21.1 & 0.91 & -28.9 & 10.3 & -18.0 & 64.0 & 61.1 & -4.56 \\
\hline 1.90 & 27.6 & 1.19 & -26.8 & 9.4 & -18.0 & 54.8 & 52.6 & -4.10 \\
\hline 1.90 & 27.6 & 1.19 & -25.5 & 0.1 & -18.0 & 47.5 & 47.2 & -0.72 \\
\hline 1.90 & 27.6 & 1.19 & -30.5 & 6.8 & -18.0 & 44.0 & 44.5 & 1.22 \\
\hline 1.90 & 27.6 & 1.19 & -29.0 & 3.2 & -18.0 & 43.2 & 45.0 & 4.24 \\
\hline 1.90 & 37.6 & 1.62 & -24.7 & -0.3 & -18.0 & 34.7 & 36.8 & 6.04 \\
\hline 1.90 & 37.6 & 1.62 & -25.3 & 1.4 & -18.0 & 39.0 & 39.2 & 0.49 \\
\hline 1.90 & 37.6 & 1.62 & -29.3 & 10.9 & -18.0 & 33.0 & 37.4 & 13.26 \\
\hline 1.90 & 37.6 & 1.62 & -28.9 & 13.1 & -18.0 & 36.3 & 38.4 & 5.86 \\
\hline 1.90 & 55.6 & 2.40 & -26.7 & 0.8 & -18.0 & 26.0 & 26.9 & 3.54 \\
\hline 1.90 & 55.6 & 2.40 & -23.3 & -0.3 & -18.0 & 29.5 & 29.3 & -0.74 \\
\hline 1.90 & 49.2 & 2.12 & -28.0 & 0.5 & -18.0 & 31.4 & 27.5 & -12.33 \\
\hline 1.90 & 49.2 & 2.12 & -30.8 & 10.4 & -18.0 & 30.0 & 28.7 & -4.33 \\
\hline 1.90 & 26.8 & 1.15 & -24.8 & 13.4 & -18.0 & 57.3 & 60.2 & 5.00 \\
\hline 1.90 & 26.8 & 1.15 & -26.2 & 10.4 & -18.0 & 55.2 & 55.6 & 0.75 \\
\hline 1.90 & 26.8 & 1.15 & -33.7 & 13.2 & -18.0 & 41.0 & 42.7 & 4.09 \\
\hline 1.90 & 26.8 & 1.15 & -31.0 & 4.6 & -18.0 & 43.0 & 43.8 & 1.75 \\
\hline 1.90 & 35.2 & 1.52 & -26.1 & 6.4 & -18.0 & 45.6 & 43.3 & -5.06 \\
\hline 1.90 & 35.2 & 1.52 & -25.7 & 3.2 & -18.0 & 45.0 & 42.3 & -6.08 \\
\hline 1.90 & 35.2 & 1.52 & -31.4 & -0.3 & -18.0 & 32.0 & 29.6 & -7.36 \\
\hline 1.90 & 35.2 & 1.52 & -29.5 & 3.1 & -18.0 & 37.2 & 36.2 & -2.80 \\
\hline 1.90 & 48.2 & 2.08 & -24.9 & 0.1 & -18.0 & 30.1 & 31.2 & 3.54 \\
\hline 1.90 & 48.2 & 2.08 & -23.8 & 10.2 & -18.0 & 35.0 & 38.8 & 10.98 \\
\hline 1.90 & 48.2 & 2.08 & -30.0 & 17.7 & -18.0 & 28.5 & 31.1 & 9.17 \\
\hline 1.90 & 48.2 & 2.08 & -30.2 & 5.9 & -18.0 & 27.8 & 28.7 & 3.29 \\
\hline 1.90 & 66.5 & 2.87 & -26.0 & 13.9 & -18.0 & 27.6 & 28.6 & 3.53 \\
\hline 1.90 & 66.5 & 2.87 & -25.4 & 10.2 & -18.0 & 28.2 & 28.7 & 1.87 \\
\hline 1.90 & 66.5 & 2.87 & -30.2 & 6.6 & -18.0 & 21.0 & 23.0 & 9.57 \\
\hline 1.90 & 66.5 & 2.87 & -29.6 & 2.4 & -18.0 & 22.2 & 22.2 & -0.05 \\
\hline
\end{tabular}


Tabla 2.26. Tiempos de congelación, experimentales y calculados. Esfera, manzanas (Ilicali \& Saglam, 1987).

\begin{tabular}{|c|c|c|c|c|c|c|c|c|}
\hline $\begin{array}{c}\mathrm{L} \\
(\mathrm{cm})\end{array}$ & $\begin{array}{c}\mathrm{h} \\
\mathrm{W} /\left(\mathrm{m}^{2 \circ} \mathrm{C}\right. \\
)\end{array}$ & $\mathrm{Bi}$ & $\begin{array}{c}\mathrm{T}_{\mathrm{a}} \\
\left({ }^{\circ} \mathrm{C}\right)\end{array}$ & $\begin{array}{c}\mathrm{T}_{\mathrm{i}} \\
\left({ }^{\circ} \mathrm{C}\right)\end{array}$ & $\begin{array}{c}\mathrm{T}_{\mathrm{c}} \\
\left({ }^{\circ} \mathrm{C}\right)\end{array}$ & $\begin{array}{c}\mathrm{t}_{\mathrm{cexp}} \\
(\mathrm{min})\end{array}$ & $\begin{array}{c}\mathrm{t}_{\text {cpre }} \\
(\mathrm{min})\end{array}$ & $\begin{array}{c}\mathrm{e} \\
(\%)\end{array}$ \\
\hline 2.50 & 13.8 & 0.88 & -15.0 & 7.0 & -10.0 & 181.2 & 205.2 & 13.23 \\
\hline 2.55 & 14.3 & 0.93 & -20.0 & 4.0 & -10.0 & 119.4 & 141.4 & 18.46 \\
\hline 2.55 & 14.3 & 0.93 & -20.0 & 4.0 & -18.0 & 165.0 & 158.5 & -3.97 \\
\hline 2.45 & 14.8 & 0.92 & -25.0 & 4.0 & -10.0 & 79.2 & 102.0 & 28.78 \\
\hline 2.45 & 14.8 & 0.92 & -25.0 & 4.0 & -18.0 & 105.0 & 114.3 & 8.82 \\
\hline 2.35 & 15.4 & 0.92 & -30.0 & 3.0 & -10.0 & 63.6 & 75.4 & 18.57 \\
\hline 2.35 & 15.4 & 0.92 & -30.0 & 3.0 & -18.0 & 84.0 & 84.5 & 0.57 \\
\hline 2.35 & 15.6 & 0.93 & -34.0 & 4.0 & -10.0 & 59.4 & 65.9 & 11.01 \\
\hline 2.35 & 15.6 & 0.93 & -34.0 & 4.0 & -18.0 & 79.2 & 73.9 & -6.73 \\
\hline 2.30 & 15.7 & 0.92 & -38.0 & 3.0 & -10.0 & 51.6 & 55.7 & 7.86 \\
\hline 2.30 & 15.7 & 0.92 & -38.0 & 3.0 & -18.0 & 65.4 & 62.4 & -4.66 \\
\hline 2.70 & 32.5 & 2.23 & -14.0 & 17.0 & -10.0 & 133.8 & 129.5 & -3.23 \\
\hline 2.70 & 33.3 & 2.29 & -20.5 & 25.0 & -10.0 & 72.6 & 84.4 & 16.25 \\
\hline 2.70 & 33.3 & 2.29 & -20.5 & 25.0 & -18.0 & 97.2 & 94.6 & -2.72 \\
\hline 2.70 & 34.1 & 2.34 & -24.5 & 23.0 & -10.0 & 66.6 & 67.5 & 1.28 \\
\hline 2.70 & 34.1 & 2.34 & -24.5 & 23.0 & -18.0 & 81.0 & 75.6 & -6.70 \\
\hline 2.80 & 34.3 & 2.44 & -29.5 & 19.0 & -10.0 & 50.4 & 56.4 & 11.94 \\
\hline 2.80 & 34.3 & 2.44 & -29.5 & 19.0 & -18.0 & 60.0 & 63.2 & 5.34 \\
\hline 2.90 & 35.3 & 2.60 & -34.5 & 24.0 & -10.0 & 45.6 & 49.4 & 8.35 \\
\hline 2.90 & 35.3 & 2.60 & -34.5 & 24.0 & -18.0 & 53.4 & 55.4 & 3.65 \\
\hline 3.20 & 47.0 & 3.83 & -14.0 & 17.0 & -10.0 & 114.0 & 126.7 & 11.13 \\
\hline 3.15 & 48.0 & 3.85 & -20.0 & 14.0 & -10.0 & 72.6 & 80.2 & 10.47 \\
\hline 3.15 & 48.0 & 3.85 & -20.0 & 14.0 & -18.0 & 97.2 & 89.9 & -7.56 \\
\hline 3.45 & 50.0 & 4.39 & -24.0 & 9.0 & -10.0 & 64.8 & 70.3 & 8.49 \\
\hline 3.45 & 50.0 & 4.39 & -24.0 & 9.0 & -18.0 & 81.0 & 78.8 & -2.77 \\
\hline 3.25 & 50.0 & 4.13 & -30.0 & 18.0 & -10.0 & 43.2 & 52.7 & 22.07 \\
\hline 3.25 & 50.0 & 4.13 & -30.0 & 18.0 & -18.0 & 49.2 & 59.1 & 20.07 \\
\hline 3.05 & 62.0 & 4.81 & -15.0 & 18.0 & -10.0 & 86.4 & 93.4 & 8.05 \\
\hline 3.15 & 61.0 & 4.89 & -20.0 & 18.0 & -10.0 & 65.4 & 71.0 & 8.58 \\
\hline 3.15 & 61.0 & 4.89 & -20.0 & 18.0 & -18.0 & 83.4 & 79.6 & -4.61 \\
\hline 3.30 & 63.0 & 5.29 & -25.0 & 17.0 & -10.0 & 48.0 & 57.8 & 20.48 \\
\hline 3.30 & 63.0 & 5.29 & -25.0 & 17.0 & -18.0 & 56.4 & 64.8 & 14.87 \\
\hline 3.50 & 63.0 & 5.61 & -30.0 & 13.0 & -10.0 & 45.0 & 50.5 & 12.23 \\
\hline 3.50 & 63.0 & 5.61 & -30.0 & 13.0 & -18.0 & 49.8 & 56.6 & 13.61 \\
\hline 3.35 & 64.6 & 5.51 & -34.0 & 12.0 & -10.0 & 37.2 & 40.4 & 8.67 \\
\hline 3.35 & 64.3 & 5.48 & -34.0 & 12.0 & -18.0 & 40.2 & 45.4 & 12.93 \\
\hline
\end{tabular}




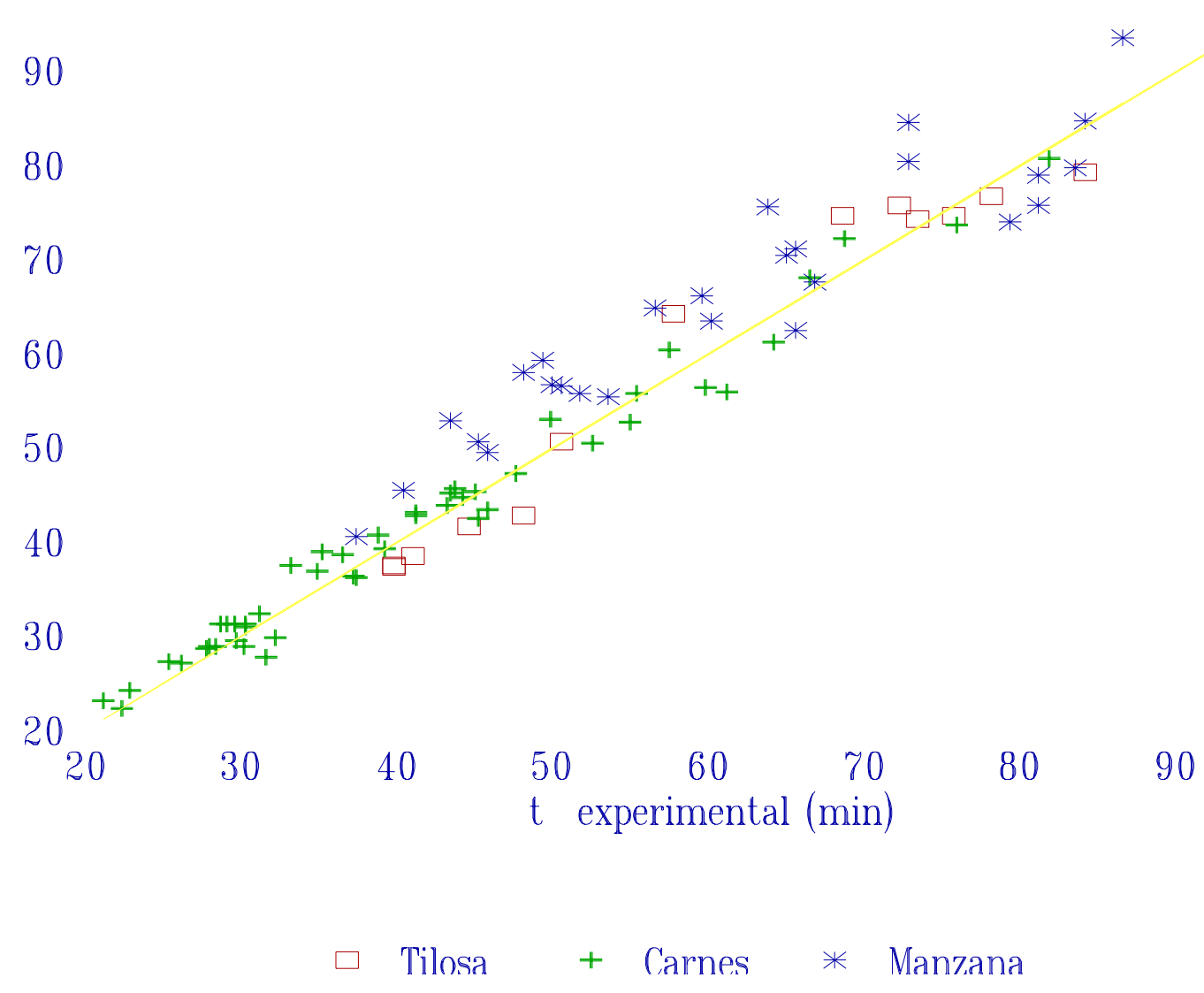

Figura 2.16a. Tiempos de congelación calculados vs. experimentales, esfera; diferenciados por tipo de producto. 
$\llbracket 50$

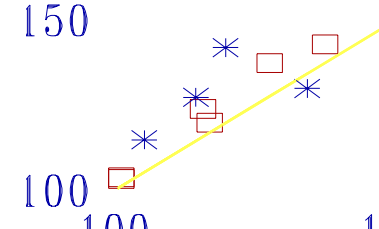

100

150

t experimental (min)

$\square$ Tilosa * Vanzana

Figura 2.16b. Tiempos de congelación calculados vs. experimentales, esfera; diferenciados por tipo de producto. 


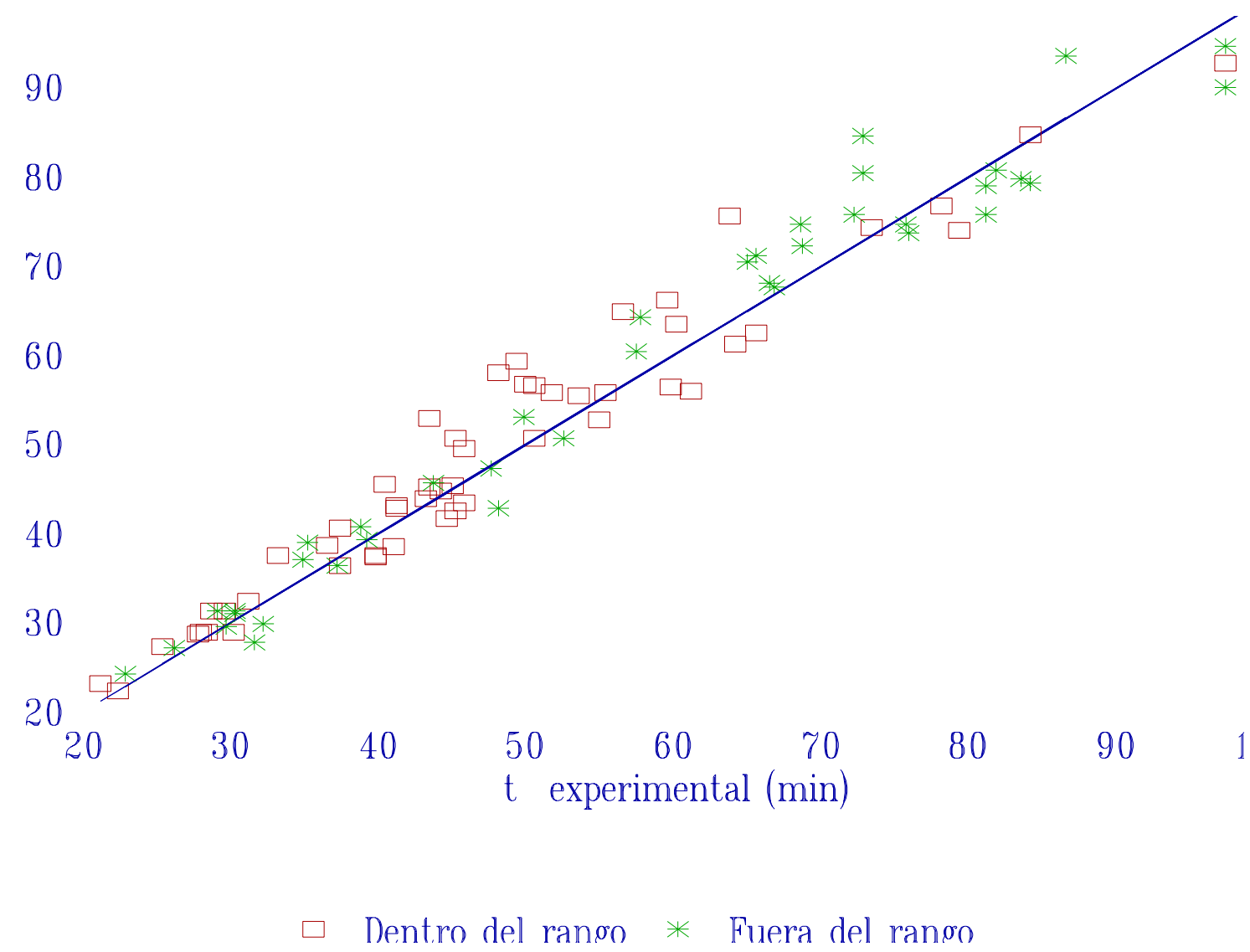

Figura 2.17a. Tiempos de congelación calculados vs. experimentales, esfera; diferenciados según sus condiciones operativas estén dentro o fuera del rango de estricta validez del método de predicción. 


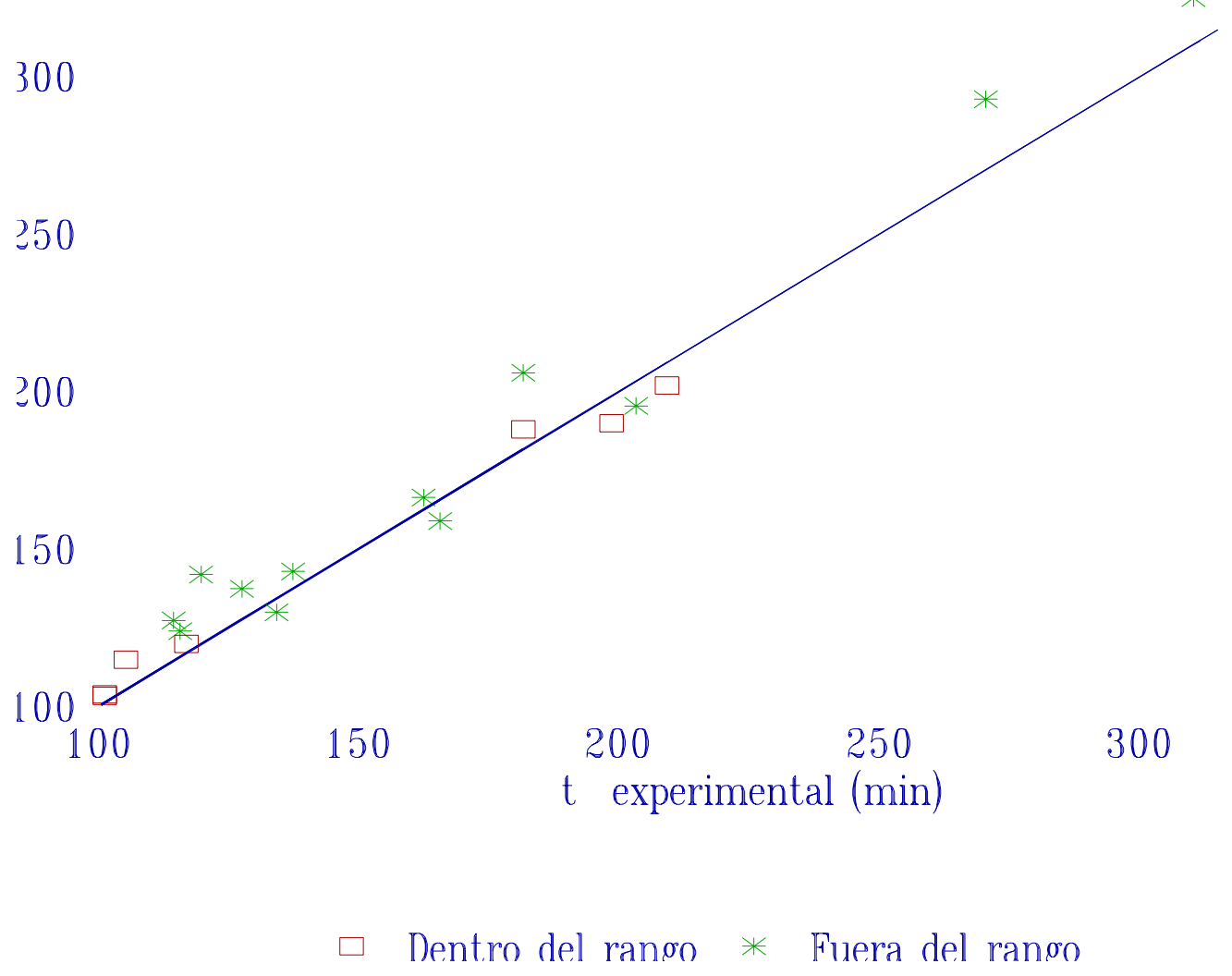

Figura 2.17b. Tiempos de congelación calculados vs. experimentales, esfera; diferenciados según sus condiciones operativas estén dentro o fuera del rango de estricta validez del método de predicción.

Como es habitual al desarrollar un método de predicción para evaluar la bondad del método se han comparado tiempos de congelación experimentales con los tiempos calculados con la metodología propuesta.

En todos los casos se ha calculado el error porcentual e(\%) definido con la siguiente ecuación: 


$$
e \%=\frac{\left(t_{\text {cpre }}-t_{\text {cexp }}\right)}{t_{\text {cexp }}} 100
$$

La Tabla 2.27 nos da el error porcentual promedio _(\%), la desviación estándar y los límites de confianza (correspondientes al 95\%), para cada geometría estudiada.

Tabla 2.27 .

\begin{tabular}{||cccccc||}
\hline \multirow{2}{*}{ Geometría } & $\begin{array}{c}\text { Número } \\
\text { de }\end{array}$ & $\begin{array}{c}\text { Error } \\
\text { Promedio } \\
(\%)\end{array}$ & $\begin{array}{c}\text { Desviación } \\
\text { Estándar } \\
(\%)\end{array}$ & $\begin{array}{c}\text { Límite } \\
\text { Superior } \\
(\%)\end{array}$ & $\begin{array}{c}\text { Límite Infe- } \\
\text { rior } \\
(\%)\end{array}$ \\
\hline \hline \multirow{2}{*}{ Placa plana } & 213 & -0.69 & 8.430 & 16.00 & -17.38 \\
Cilindro infinito & 36 & -2.24 & 6.248 & 10.26 & -14.74 \\
Esfera & 114 & 3.41 & 7.522 & 18.45 & -11.64 \\
Todos los datos & 363 & 0.44 & 8.203 & 16.84 & -15.96 \\
\hline \hline
\end{tabular}

\subsubsection{RESULTADOS DE CONGELACION ASIMETRICA.}

En las Tablas 2.28 a 2.30 se presentan los conjuntos de datos experimentales correspondientes a congelación de placa plana infinita, bajo condiciones externas asimétricas. En cada corrida experimental se detallan dos coeficientes de transferencia calórica y dos temperaturas de refrigerante, observándose una o las dos asimetrías. Para cada caso se han calculado tres longitudes eqivalentes: Leq1 según la metodología de De Michelis, Leq2 según la propuesta de Pham, y por último Leq3 de acuerdo a la metodología desarrollada en este trabajo. Se observa que las tres metodologías conducen a semiespesores equivalentes muy similares, es esperable obtener los mejores resultados calculando el tiempo de congelación con la ecuación propuesta por el autor del método de cálculo del semiespesor respectivo.

Luego de evaluar las longitudes equivalentes se calcularon los tiempos de 
congelación respectivos, con la ec. (2.55), utilizando la longitud equivalente correspondiente. En estas tablas tc1 es el tiempo de congelación calculado con Leq1, tc2 el tiempo correspondiente al semiespesor Leq2 $\mathrm{y}$, por último, tc3 es el tiempo de congelación del alimento de semiespesor equivalente Leq3. Como se observa que el error porcentual es elevado en muchos casos, en la tabla también se presenta el tiempo de congelación tcnum, calculado para cada situación mediante un modelo numérico de diferencias finitas, comprobándose que el error es del mismo orden que el correspondiente al método de predicción simplificado. Este se debe a que las medidas experimentales disponibles no son demasiado buenas, tienen un gran error experimental.

Tabla 2.28. Tiempos de congelación, experimentales y calculados. Placa plana, carne vacuna (De Michelis \& Calvelo, 1982).

\begin{tabular}{|c|c|c|c|c|c|c|c|c|c|}
\hline $\begin{array}{c}\text { Corri- } \\
\text { da }\end{array}$ & $\begin{array}{c}\mathrm{D} \\
(\mathrm{cm})\end{array}$ & $\begin{array}{c}\mathrm{h}_{1} \\
\mathrm{~W} /\left(\mathrm{m}^{2^{\circ} \mathrm{C}} \mathrm{C}\right. \\
) \\
\end{array}$ & $\begin{array}{c}\mathrm{h}_{2} \\
\mathrm{~W} /\left(\mathrm{m}^{2{ }^{\circ}} \mathrm{C}\right. \\
) \\
\end{array}$ & $\begin{array}{c}\mathrm{T}_{\mathrm{a} 1} \\
\left({ }^{\circ} \mathrm{C}\right)\end{array}$ & $\begin{array}{c}\mathrm{T}_{\mathrm{a} 2} \\
\left({ }^{\circ} \mathrm{C}\right)\end{array}$ & $\begin{array}{c}\mathrm{T}_{\mathrm{i}} \\
\left({ }^{\circ} \mathrm{C}\right)\end{array}$ & $\begin{array}{l}\text { Leq1 } \\
(\mathrm{cm})\end{array}$ & $\begin{array}{l}\text { Leq2 } \\
(\mathrm{cm})\end{array}$ & $\begin{array}{l}\text { Leq3 } \\
(\mathrm{cm})\end{array}$ \\
\hline 1 & 6.00 & 168.7 & 168.7 & -40.0 & -35.0 & 10.0 & 3.25 & 3.22 & 3.18 \\
\hline 2 & 6.00 & 169.4 & 169.4 & -39.0 & -33.0 & 9.0 & 3.25 & 3.18 & 3.24 \\
\hline 3 & 6.00 & 151.0 & 151.051 .9 & -40.0 & -35.0 & 8.0 & 3.23 & 3.15 & 3.18 \\
\hline 4 & 10.00 & 103.8 & 20.8 & -42.0 & -42.0 & 15.0 & 5.50 & 5.50 & 5.50 \\
\hline 5 & 9.40 & 28.8 & & -43.0 & -43.0 & 15.0 & 5.17 & 5.10 & 5.17 \\
\hline
\end{tabular}

Tabla 2.28. Continuación.

\begin{tabular}{|c|c|c|c|c|c|c|c|c|c|}
\hline $\begin{array}{c}\text { Corri- } \\
\text { da }\end{array}$ & $\begin{array}{c}t_{\text {cexp }} \\
(\mathrm{min})\end{array}$ & $\begin{array}{c}\mathrm{t}_{\mathrm{c} 1} \\
(\mathrm{~min})\end{array}$ & $\begin{array}{c}\mathrm{e} \\
(\%) \\
\end{array}$ & $\begin{array}{c}t_{\mathrm{c} 2} \\
(\mathrm{~min}) \\
\end{array}$ & $\begin{array}{c}\mathrm{e} \\
(\%) \\
\end{array}$ & $\begin{array}{c}\mathrm{t}_{\mathrm{c} 3} \\
(\mathrm{~min})\end{array}$ & $\begin{array}{c}\mathrm{e} \\
(\%)\end{array}$ & $\begin{array}{r}t_{\text {cnum }} \\
(\mathrm{min}) \\
\end{array}$ & $\begin{array}{c}\mathrm{e} \\
(\%) \\
\end{array}$ \\
\hline 1 & 69.0 & 78.3 & 13.47 & 77.2 & 11.94 & 75.6 & 9.61 & 76.4 & 10.80 \\
\hline 2 & 69.4 & 79.7 & 14.86 & 77.0 & 10.95 & 79.4 & 14.50 & 79.5 & 14.62 \\
\hline 3 & 67.2 & 78.9 & 17.39 & 75.8 & 12.73 & 77.0 & 14.51 & 78.4 & 16.62 \\
\hline 4 & 240.0 & 218.0 & -9.15 & 218.0 & -9.15 & 218.0 & -9.15 & 225.3 & -6.12 \\
\hline 5 & 305.0 & 349.9 & 14.73 & 343.4 & 12.60 & 349.9 & 14.73 & 365.0 & 19.67 \\
\hline
\end{tabular}


Tabla 2.29. Tiempos de congelación, experimentales y calculados. Placa plana, cordero (Bazán \& Mascheroni, 1984).

\begin{tabular}{|c|c|c|c|c|c|c|c|c|c|}
\hline $\begin{array}{c}\text { Corri- } \\
\text { da }\end{array}$ & $\begin{array}{c}\mathrm{D} \\
(\mathrm{cm})\end{array}$ & $\begin{array}{c}\mathrm{h}_{1} \\
\mathrm{~W} /\left(\mathrm{m}^{2 \circ} \mathrm{C}\right. \\
)\end{array}$ & $\begin{array}{c}\mathrm{h}_{2} \\
\mathrm{~W} /\left(\mathrm{m}^{2 \circ} \mathrm{C}\right. \\
)\end{array}$ & $\begin{array}{c}\mathrm{T}_{\mathrm{a} 1} \\
\left({ }^{\circ} \mathrm{C}\right)\end{array}$ & $\begin{array}{c}\mathrm{T}_{\mathrm{a} 2} \\
\left({ }^{\circ} \mathrm{C}\right)\end{array}$ & $\begin{array}{c}\mathrm{T}_{\mathrm{i}} \\
\left({ }^{\circ} \mathrm{C}\right)\end{array}$ & $\begin{array}{l}\text { Leq1 } \\
(\mathrm{cm})\end{array}$ & $\begin{array}{l}\text { Leq2 } \\
(\mathrm{cm})\end{array}$ & $\begin{array}{l}\text { Leq3 } \\
(\mathrm{cm})\end{array}$ \\
\hline 1 & 6.00 & 78.2 & 80.8 & -37.7 & -36.3 & 10.6 & 3.03 & 3.03 & 3.03 \\
\hline 2 & 6.00 & 111.7 & 87.4 & -40.2 & -40.1 & 9.6 & 3.12 & 3.12 & 3.12 \\
\hline 3 & 6.00 & 117.1 & 124.2 & -33.2 & -33.1 & 10.3 & 2.94 & 2.98 & 2.94 \\
\hline 4 & 6.00 & 81.3 & 124.7 & -40.1 & -39.7 & 9.4 & 2.76 & 2.81 & 2.76 \\
\hline 5 & 6.00 & 105.0 & 248.1 & -32.5 & -31.9 & 8.6 & 2.66 & 2.73 & 2.69 \\
\hline 6 & 6.00 & 181.2 & 178.2 & -31.5 & -30.8 & 16.5 & 3.03 & 3.03 & 3.04 \\
\hline 7 & 6.00 & 58.2 & 251.5 & -31.8 & -31.6 & 3.8 & 2.28 & 2.38 & 2.28 \\
\hline 8 & 6.00 & 172.0 & 244.8 & -25.5 & -22.4 & 2.7 & 3.12 & 3.05 & 3.24 \\
\hline 9 & 10.00 & 52.3 & 192.5 & -38.1 & -38.0 & 10.6 & 4.20 & 4.28 & 4.20 \\
\hline 10 & 10.00 & 279.1 & 320.5 & -38.0 & -38.0 & 11.2 & 5.00 & 4.97 & 5.00 \\
\hline 11 & 10.00 & 210.5 & 343.1 & -39.9 & -31.0 & 2.8 & 5.33 & 5.30 & 5.43 \\
\hline 12 & 10.00 & 121.8 & 287.9 & -29.8 & -28.5 & 2.7 & 4.84 & 4.79 & 4.82 \\
\hline 13 & 10.00 & 160.2 & 58.2 & -32.1 & -32.0 & 10.8 & 5.67 & 5.58 & 5.67 \\
\hline 14 & 10.00 & 102.5 & 289.2 & -25.7 & -24.9 & 2.4 & 4.66 & 4.69 & 4.75 \\
\hline 15 & 10.00 & 333.1 & 156.1 & -25.6 & -22.4 & 10.5 & & 5.44 & 5.85 \\
\hline
\end{tabular}

Tabla 2.29. Continuación.

\begin{tabular}{|c|c|c|c|c|c|c|c|c|c|}
\hline $\begin{array}{c}\text { Corri- } \\
\text { da }\end{array}$ & $\begin{array}{c}\text { tcexp } \\
\text { (min) }\end{array}$ & $\begin{array}{c}\mathrm{t}_{\mathrm{c} 1} \\
(\mathrm{~min})\end{array}$ & $\begin{array}{c}\mathrm{e} \\
(\%)\end{array}$ & $\begin{array}{c}\mathbf{t}_{\mathrm{c} 2} \\
(\mathrm{~min})\end{array}$ & $\begin{array}{c}\mathrm{e} \\
(\%)\end{array}$ & $\begin{array}{c}\mathrm{t}_{\mathrm{c} 3} \\
(\mathrm{~min})\end{array}$ & $\begin{array}{c}\mathrm{e} \\
(\%)\end{array}$ & $\begin{array}{c}\mathrm{t}_{\mathrm{cnum}} \\
(\mathrm{min})\end{array}$ & $\begin{array}{c}\mathrm{e} \\
(\%)\end{array}$ \\
\hline 1 & 114.0 & 114.8 & 0.71 & 114.8 & 0.71 & 114.8 & 0.71 & 116.9 & 2.56 \\
\hline 2 & 88.0 & 93.3 & 6.05 & 93.3 & 6.05 & 93.3 & 6.05 & 95.4 & 8.43 \\
\hline 3 & 115.5 & 103.5 & -10.42 & 105.7 & -8.51 & 103.5 & -10.42 & 108.4 & -6.13 \\
\hline 4 & 99.0 & 90.7 & -8.37 & 93.1 & -5.95 & 90.7 & -8.37 & 95.6 & -3.48 \\
\hline 5 & 106.0 & 94.0 & -11.35 & 97.8 & -7.70 & 95.6 & -9.84 & 101.0 & -4.69 \\
\hline 6 & 145.0 & 101.6 & -29.94 & 101.6 & -29.94 & 101.9 & -29.71 & 104.1 & -28.19 \\
\hline 7 & 124.0 & 102.1 & -17.63 & 108.2 & -12.77 & 102.1 & -17.63 & 115.9 & -6.55 \\
\hline 8 & 157.0 & 118.2 & -24.72 & 113.8 & -27.51 & 125.9 & -19.82 & 126.3 & -19.55 \\
\hline 9 & 240.0 & 227.2 & -5.34 & 233.5 & 2.72 & 227.2 & -5.34 & 238.6 & -0.57 \\
\hline 10 & 209.0 & 174.0 & -16.77 & 172.0 & -17.68 & 174.0 & -16.77 & 174.7 & -16.41 \\
\hline 11 & 228.0 & 174.1 & -23.63 & 172.3 & -24.41 & 180.1 & -21.02 & 182.4 & -19.98 \\
\hline 12 & 264.5 & 232.0 & -12.27 & 228.0 & -13.79 & 230.4 & -12.88 & 247.4 & -6.46 \\
\hline 13 & 287.0 & 291.8 & 1.68 & 283.7 & -1.16 & 291.8 & 1.68 & 287.7 & 0.23 \\
\hline 14 & 340.0 & 269.9 & -20.61 & 272.8 & -19.77 & 278.5 & -18.08 & 303.1 & -10.84 \\
\hline 15 & 363.0 & & & 305.1 & -15.94 & 349.6 & -3.68 & 319.3 & -12.03 \\
\hline
\end{tabular}


Tabla 2.30. Tiempos de congelación, experimentales y calculados. Placa plana, carne picada, $Y_{0}=0.68$ (Flores \& Mascheroni, 1984).

\begin{tabular}{|c|c|c|c|c|c|c|c|c|c|}
\hline $\begin{array}{c}\text { Corri- } \\
\text { da }\end{array}$ & $\begin{array}{c}\mathrm{D} \\
(\mathrm{cm})\end{array}$ & $\begin{array}{c}\mathrm{h}_{1} \\
\mathrm{~W} /\left(\mathrm{m}^{2 \circ} \mathrm{C}\right. \\
)\end{array}$ & $\begin{array}{c}\mathrm{h}_{2} \\
\mathrm{~W} /\left(\mathrm{m}^{2 \circ} \mathrm{C}\right. \\
)\end{array}$ & $\begin{array}{l}\mathrm{T}_{\mathrm{a} 1} \\
\left({ }^{\circ} \mathrm{C}\right)\end{array}$ & $\begin{array}{c}\mathrm{T}_{\mathrm{a} 2} \\
\left({ }^{\circ} \mathrm{C}\right)\end{array}$ & $\begin{array}{c}\mathrm{T}_{\mathrm{i}} \\
\left({ }^{\circ} \mathrm{C}\right)\end{array}$ & $\begin{array}{l}\text { Leq1 } \\
(\mathrm{cm})\end{array}$ & $\begin{array}{l}\text { Leq2 } \\
(\mathrm{cm})\end{array}$ & $\begin{array}{l}\text { Leq3 } \\
(\mathrm{cm})\end{array}$ \\
\hline 1 & 6.00 & 350.0 & 54.5 & -25.6 & -19.2 & 8.6 & & 4.02 & 4.68 \\
\hline 2 & 6.00 & 247.5 & 65.6 & -25.5 & -24.5 & 5.2 & 3.61 & 3.56 & 3.66 \\
\hline 3 & 6.00 & 302.3 & 172.3 & -25.4 & -20.1 & 5.0 & 3.43 & 3.39 & 4.08 \\
\hline 4 & 6.00 & 200.3 & 200.9 & -25.6 & -21.1 & 16.2 & 3.17 & 3.21 & 3.57 \\
\hline 5 & 8.00 & 255.8 & 335.9 & -26.6 & -24.2 & 3.0 & & 4.08 & 4.24 \\
\hline 6 & 8.00 & 18.0 & 412.0 & -27.8 & -23.9 & 3.6 & 2.16 & 2.46 & 2.52 \\
\hline 7 & 8.00 & 417.2 & 243.7 & -25.8 & -22.7 & 10.0 & & 4.27 & 4.62 \\
\hline
\end{tabular}

Tabla 2.30. Continuación.

\begin{tabular}{|c|c|c|c|c|c|c|c|c|c|}
\hline $\begin{array}{c}\text { Corri- } \\
\text { da }\end{array}$ & $\begin{array}{c}\text { tcexp } \\
\text { (min) }\end{array}$ & $\begin{array}{c}\mathrm{t}_{1} 1 \\
(\mathrm{~min})\end{array}$ & $\begin{array}{c}\mathrm{e} \\
(\%)\end{array}$ & $\begin{array}{c}\mathrm{t}_{\mathrm{c} 2} \\
(\mathrm{~min})\end{array}$ & $\begin{array}{c}\mathrm{e} \\
(\%)\end{array}$ & $\begin{array}{c}\mathrm{t}_{\mathrm{c} 3} \\
(\mathrm{~min})\end{array}$ & $\begin{array}{c}\mathrm{e} \\
(\%)\end{array}$ & $\begin{array}{c}t_{\text {cnum }} \\
(\mathrm{min})\end{array}$ & $\begin{array}{c}\mathrm{e} \\
(\%)\end{array}$ \\
\hline 1 & 163.2 & & & 157.6 & -3.44 & 209.3 & 28.22 & 174.0 & 6.62 \\
\hline 2 & 135.0 & 132.6 & $-1.76 \|$ & 129.2 & -4.28 & 135.8 & 0.59 & 141.0 & 4.44 \\
\hline 3 & 120.0 & 116.1 & $-3.29 \|$ & 113.8 & -5.13 & 159.7 & 33.08 & 127.8 & 6.50 \\
\hline 4 & 163.8 & 122.0 & -25.52 & 124.4 & -24.05 & 149.7 & -8.61 & 137.4 & 16.12 \\
\hline 5 & 180.0 & & & 150.2 & -16.58 & 161.0 & -10.53 & 168.6 & -6.33 \\
\hline 6 & 270.0 & 234.1 & -13.29 & 271.9 & 0.69 & 279.6 & 3.54 & 312.0 & 15.56 \\
\hline 7 & 167.4 & & & 173.2 & 3.46 & 200.0 & 19.85 & 186.6 & 11.47 \\
\hline
\end{tabular}

En la Figura 2.18 se han graficado los tiempos de congelación predichos $t_{c} 3$ vs. los valores experimentales, correspondientes a los tres juegos de datos anteriores.

Para calcular la precisión del método de predicción, en las Tablas 2.28, 2.29 y 2.30 se detalla el error porcentual e, definido por la ec. (2.63), para los tiempos de congelación calculados por los cuatro esquemas.

En la Tabla 2.30 se detallan el error porcentual promedio _ para cada alternativa y la desviación estándar. En el caso de la alternativa 1 se computan 23 corridas, ya que en las cuatro restantes no pudo efectuarse el cálculo debido a que las condiciones operativas caían fuera del rango de la gráfica utilizada, para las restantes alternativas el error promedio y la desviación estándar se calculan con 27 datos. 


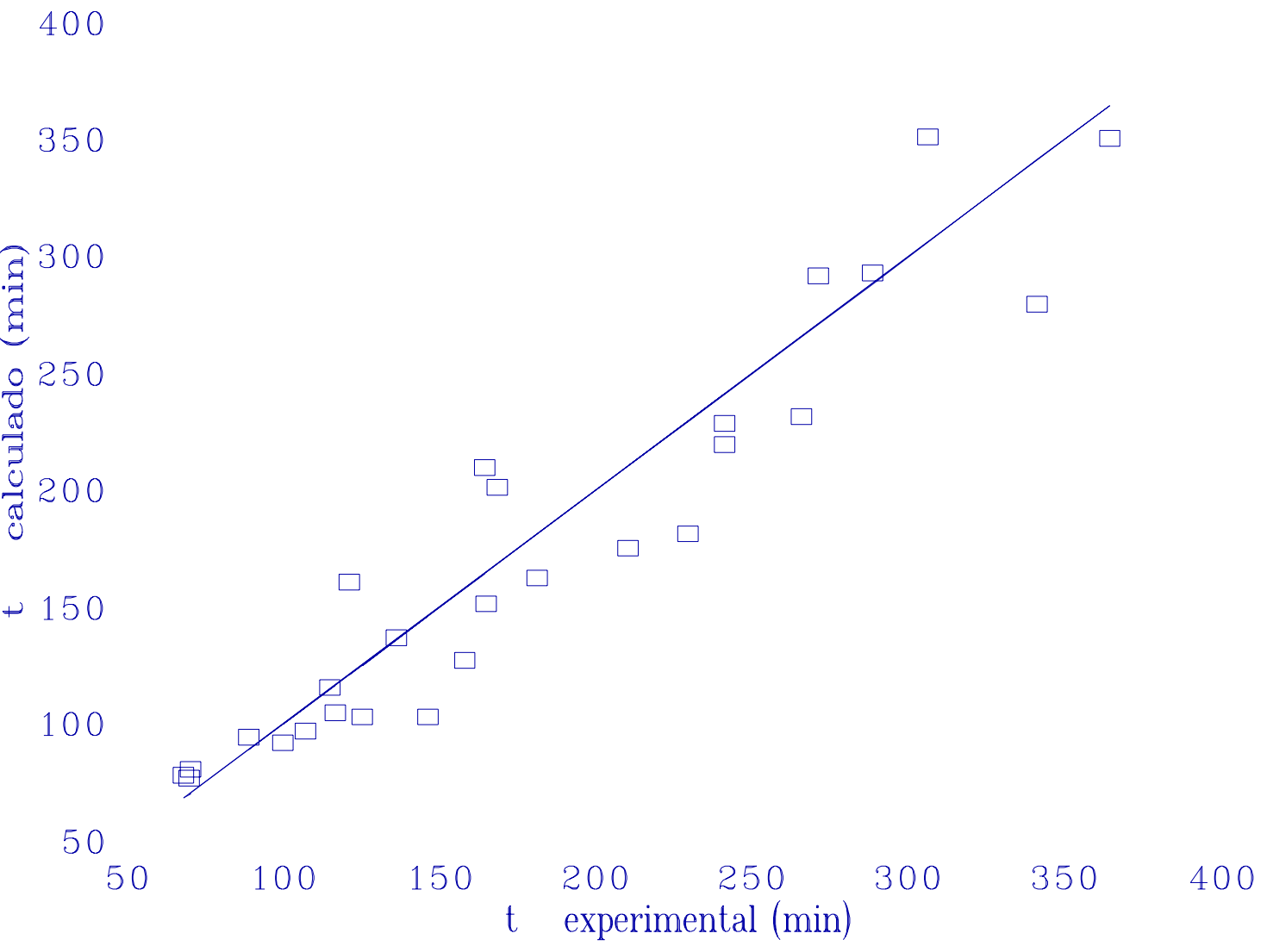

Figura 2.18. Tiempos de congelación de placa plana, carnes, congeladas bajo condiciones operativas asimétricas.

Tabla 2.30. Error porcentual promedio y desviación estándar correspondientes a los tiempos de congelación asimétrica predichos.

\begin{tabular}{||ccccc||}
\hline & & & & \\
& Esquema & Esquema & Esquema & Método \\
& 1 & 2 & 3 & numérico \\
\hline \hline$(\%)$ & -7.18 & -7.09 & -2.03 & -3.16 \\
Desv. Estándar & 13.932 & 12.413 & 15.622 & 12.221 \\
\hline \hline
\end{tabular}




\subsection{CONCLUSIONES}

Se ha desarrollado un método de predicción de tiempos de congelación de alimentos, válido para las formas regulares, cuya fórmula de cálculo es extremadamente simple, siendo necesario conocer sólo dos propiedades termofísicas del producto sin congelar (la difusividad térmica $\alpha_{0}$ y la conductividad térmica $\mathrm{k} 0$ ) .

Se ha probado que es válido para un rango amplísimo de condiciones operativas, simétricas o asimétricas, cubriendo extensamente las condiciones más frecuentes en la congelación industrial.

Se ha probado también que el método es válido para una gran variedad de alimentos, de composición y estructura diferentes como carne vacuna entera y picada, distintos pescados, productos hortifrutícolas, geles, etc. En general el método podrá aplicarse a alimentos de alto contenido inicial de agua. 


\title{
CAPITULO III
}

\author{
PREDICCION DE TIEMPOS DE \\ DESCONGELACION DE ALIMENTOS
}




\subsection{INTRODUCCION}

La mayoría de los alimentos que se congelan, se venden congelados y son descongelados en el hogar por los consumidores. En estos casos no tiene sentido analizar desde un punto de vista técnico el proceso de descongelación. Pero en la actualidad muchas veces se congelan alimentos (como productos intermedios) para preservarlos hasta que se realiza el procesamiento definitivo. Aquí sí se practica la descongelación a escala industrial, y análogamente al proceso de congelación el tiempo de duración del proceso es una de las variables de mayor interés, ya que el mismo influye en aspectos de la calidad del alimento como son la pérdida de "jugo" por exudado o evaporación y el crecimiento microbiano en la superficie debido a que ésta alcanza al tas temperaturas.

La descongelación se produce generalmente por alguno de los dos mecanismos siguientes: calentamiento por microondas o calentamiento convectivo, utilizando aire o agua caliente. En el presente trabajo no analizaremos la descongelación con microondas, nos limitaremos al estudio del calentamiento convectivo.

\subsection{EL PROCESO DE DESCONGELACION}

Un planteamiento muy simple consideraría que la descongelación es exactamente el proceso inverso a la congelación, y entonces, sería válido utilizar los métodos de predicción desarrollados para modelar la congelación para predecir los tiempos de descongelación.

La principal diferencia reside en que en la congelación el calor extraído al al imento debe atravesar una zona ya congelada mientras que al descongelar el flujo de calor se transfiere a través del alimento descongelado, de menor conductividad térmica. Por lo tanto, frente a igual fuerza impulsora, el tiempo de descongelación será mayor queel de congelación.

\subsection{METODOS DE PREDICCION}

\subsubsection{SOLUCIONES EXACTAS}


Los métodos de cálculo del tiempo de descongelación pueden dividirse en métodos "exactos" y aproximados. En el caso de los métodos exactos valen las consideraciones hechas al analizar la congelación, los mismos autores que desarrollaron modelos numéricos para estudiar la congelación hicieron lo propio con la descongelación. El modelo numérico deberá resolver el balance diferencial de energía térmica, planteado en la ec. (2.28) con las mismas condiciones de contorno, sólo que ahora la temperatura inicial $\mathrm{Ti}_{\mathrm{i}}$ generalmente es inferior a $-15^{\circ} \mathrm{C}$ y la temperatura del medio externo $\mathrm{T}_{\mathrm{a}}$ será bastante superior $\mathrm{a} 0^{\circ} \mathrm{C}$.

Schwartzberg y col. (1977) utilizan métodos numéricos para predecir perfiles de temperatura durante la descongelación, pero no comparan los resultados con valores experimentales.

Bailey y col. (1974), James y col. (1977) y James \& Bailey (1980), utilizan un método numérico explícito simplificado para predecir tiempos de descongelación, aplicándolo a alimentos de distintas geometrías obteniendo buenos resultados. No presentan perfiles de temperatura, probablemente debido a que al ser el método simplificado los valores puntuales de temperatura no son muy exactos, principalmente en la zona de cambio de fase.

Mascheroni (1982) estudia la descongelación con dos esquemas numéricos: uno explícito y otro implícito tipo Crank-Nicolson. Los resultados se comparan con valores experimentales para dos situaciones: descongelación por vapor de agua en vacío (h entre 1000 y $6000 \mathrm{~W} /\left(\mathrm{m}^{2 \circ} \mathrm{C}\right)$ ) y túneles de descongelación con flujo de aire forzado ( $h$ entre 15 y $40 \mathrm{~W} /\left(\mathrm{m}^{2 \circ} \mathrm{C}\right)$ ), utilizando bloques de carne picada.

Flores \& Mascheroni (1983) comparan tres métodos de diferencias finitas: un esquema de Lees, un esquema implícito de direcciones alternantes y un método explícito, para modelar la descongelación de bloques de productos alimenticios por aspersión de agua. Con los dos primeros esquemas los autores tienen problemas de estabilidad, utilizan el tercero, verificando su bondad comparando los resultados obtenidos con propiedades constantes con la aplicación de la regla de N ewmann. Se verifica el modelo con propiedades variables con resultados experimentales de muestras degel deágar. 
Cleland y col. (1986) utilizan diferencias finitas (esquema de Lees) y elementos finitos para modelar la descongelación de las tres formas regulares (placa plana infinita, cilindro infinito y esfera), considerando condición de contorno convectiva (de tercer tipo). Los mismos autores presentan un conjunto de datos experimentales obtenidos para verificar la validez de ambos modelos numéricos, comprobando que para definir el tamaño de la grilla y el incremento de tiempo, ambos fenómenos (congelación y descongelación) son equivalentes.

Mannapperuma \& Singh (1988b) estudian la descongelación con aire húmedo, analizando la variación del coeficiente de transferencia calórica producida debido a que asociado al fenómeno de transferencia de calor se produce transferencia de masa. Desarrollan un modelo matemático que plantea el comportamiento de la superficie durante la descongelación identificando seis etapas, cada una con un valor de $\mathrm{h}$ determinado: formación de escarcha, consolidación de la escarcha, etapa de transición (la escarcha se funde, la temperatura de la superficie se mantiene constante), condensación, evaporación y secado.

\subsubsection{SOLUCIONES APROXIMADAS}

Vanichseni (1971) modifica la ecuación de Plank, reemplazando el calor latente por el cambio total de ental pía. Se limita a la descongelación de piernas de cordero, presentando un nomograma que permite el cálculo del tiempo de descongelación td en función del peso de la res, cuando el medio de calefacción es agua. El gráfico está construído para $T_{a}=113^{\circ} \mathrm{F}$, pero contempla otras temperaturas mediante un factor corrector.

El mismo autor estudió experimentalmente la descongelación con aire caliente, los resultados también se presentan como un nomograma, construído para $\mathrm{Ti}=12^{\circ} \mathrm{C}$ y $\mathrm{Ta}=10^{\circ} \mathrm{C}$; por medio de dos factores de corrección pueden considerarse diferentes temperaturas inicial y del medio externo, respectivamente.

James y col. (1976) desarrollan un método de predicción de td basado en la determinación experimental del punto final de cambio de fase en el alimento que está siendo descongelado. Este punto va acompañado por un notable incremento de 
la temperatura de la superficie del alimento. Los autores grafican ( $\left.\Delta \log \left[T_{a}-T_{s}\right] / \Delta t\right)$ en función del tiempo, la ordenada es constante durante el cambio de fase y aumenta rápidamente una vez que el centro se ha descongelado. Se ha definido a td como el tiempo en que la ordenada supera en $2.5 \mathrm{el}$ valor constante. Este método ha sido verificado con valores experimentales logrando buena concordancia. Es válido para descongelación con aire ( $h$ entre 10 y $90 \mathrm{~W} /\left(\mathrm{m}^{20} \mathrm{C}\right)$ ), ya que con mayores coeficientes de transferencia calórica el ascenso de temperatura de la superficie no es muy notable.

Creed \& James (1981) estudian la descongelación de bloques, con transferencia de calor en una sola dimensión, mediante un modelo numérico. Regresionando los resultados, obtienen una ecuación simplificada para el cál culo de td:

$$
t_{d}=\frac{D^{2.043 T_{a}^{0.012}}}{3.947+0.43 T_{a}}+\frac{123.724 T_{a}^{-0.831} D^{0.928 T_{a}^{0.059}}}{h}
$$

La ecuación anterior es válida para el siguiente rango de condiciones operativas:

Tabla 3.1. Rango de validez de la ec. (3.1).

\begin{tabular}{||cccc||}
\hline $\begin{array}{c}\mathrm{D} \\
(\mathrm{cm})\end{array}$ & $\begin{array}{c}\mathrm{Ti} \\
\left({ }^{\circ} \mathrm{C}\right)\end{array}$ & $\begin{array}{c}\mathrm{Ta} \\
\left({ }^{\circ} \mathrm{C}\right)\end{array}$ & $\begin{array}{c}\mathrm{h} \\
\left(\mathrm{W} /\left(\mathrm{m}^{2 \circ} \mathrm{C}\right)\right)\end{array}$ \\
\hline \hline $13 \mathrm{a} 16$ & -30 & $5 \mathrm{a} \mathrm{30}$ & $5 \mathrm{a} 1000$ \\
\hline
\end{tabular}

Levy (1983) plantea una ecuación que relaciona la temperatura del centro

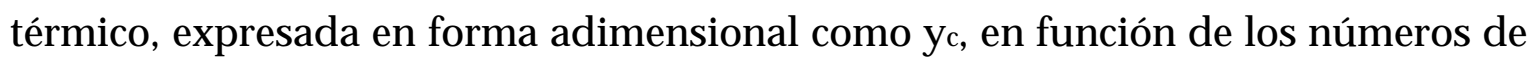
Biot y Fourier:

$$
\ln y_{c}=\ln \frac{T_{a}-T_{c}}{T_{a}-T_{i}}=-\frac{1.025 F o}{0.474+\frac{1}{B i}}
$$


Fijando la temperatura final deseada $T_{c}, y$ desadimensionalizando el número de Fourier, puede calcularse el tiempo de descongelación. Esta metodología no ha sido contrastada con resultados experimentales.

Cleland y col. (1986) presentan cuatro ecuaciones de cálculo del tiempo de descongelación, basándose en métodos desarrollados para congelación por ellos mismos o por otros autores. Los parámetros de ajuste de las distintas ecuaciones se obtuvieron regresionando resultados experimentales de descongelación, de las tres geometrías, cubriendo el rango de condiciones operativas de la Tabla 3.2.

Tabla 3.2. Condiciones operativas barridas experimentalmente por Cleland y col. (1986).

\begin{tabular}{|c|c|c|c|}
\hline $\begin{array}{l}\text { Tc } \\
\left({ }^{\circ} \mathrm{C}\right)\end{array}$ & $\begin{array}{c}\mathrm{T}_{\mathrm{i}} \\
\left({ }^{\circ} \mathrm{C}\right)\end{array}$ & $\begin{array}{l}\mathrm{Ta}_{\mathrm{a}} \\
\left({ }^{\circ} \mathrm{C}\right)\end{array}$ & $\begin{array}{c}h \\
\left(W /\left(m^{2 \circ} \mathrm{C}\right)\right)\end{array}$ \\
\hline & $-10 a-33$ & 5 a 45 & 13 a 246 \\
\hline
\end{tabular}

La ec. (3.3) se basa en una formulación desarrollada por Mascheroni citada en Calvelo (198):

$$
t_{d}=2.8582 \frac{D}{\alpha} \frac{V}{A}\left(\frac{0.5}{B^{\prime} i S t e}+\frac{0.125}{S t e}\right)^{1.0248} S t e^{0.2712} P k^{0.061}
$$

donde Fo es el número de Fourier, Bi' el número de Biot, Ste el número de Stefan y Pk el número de Plank, ya definidos en el Capítulo II (ecs. (2.11), (2.12) y (2.13)).

Otra opción es una modificación de la ecuación original de Plank, regresionando los factores de forma $\mathrm{P}$ y $\mathrm{R}$ de modo similar a lo efectuado para congelación para las distintas geometrías:

$$
t_{d}=\frac{2 D}{\alpha} \frac{V}{A}\left(\frac{P}{B^{\prime} i S t e}+\frac{R}{S t e}\right)
$$

donde: 


$$
\begin{gathered}
P=0.5(0.7754+2.2828 \text { StePk }) \\
R=0.125\left(0.4271+2.1220 \text { Ste }-1.4847 S_{t e}^{2}\right)
\end{gathered}
$$

Una tercera ecuación surge de plantear un esquema similar al propuesto por Pham (1984) para predecir tiempos de congelación:

$$
t_{d}=\frac{2 V}{A D} \sum_{1}^{3} \frac{\Delta H_{i}^{*} D}{\Delta T_{i} 2 h}\left(1+\frac{h D}{4 k_{i}}\right)
$$

donde:

$$
\begin{aligned}
& \Delta H_{l}^{*}=\rho_{s} C_{p s}\left(T_{f m}-T_{i}\right) \quad \text { y } \quad \Delta T_{l}=T_{a}-0.5\left(T_{i}+T_{f m}\right) \quad y \quad k_{l}=k_{s} \\
& \Delta H_{2}^{*}=\lambda_{f, e f}^{*} \quad y \quad \Delta T_{2}=T_{a}-T_{f m} \quad y \quad k_{2}=0.25 k_{s}+.75 k_{0} \\
& \Delta H_{3}^{*}=\rho_{0} C_{p 0}\left(T_{0}-T_{f m}\right) \quad \text { y } \Delta T_{3}=T_{a}-0.5\left(T_{0}+T_{f m}\right) \text { y } k_{3}=k_{0} \\
& T_{0}=T_{c}-\frac{\left(T_{c}-T_{a}\right)}{2+\frac{4}{B i}} \quad y \quad T_{f m}=T_{f}-1.5
\end{aligned}
$$

La cuarta modificación a la ec. de Plank también se basa en una propuesta realizada por Pham para el cálculo de los tiempos de congelación:

$$
t_{d}=\frac{2 D}{\alpha} \frac{V}{A}\left(\frac{0.5}{B^{\prime} i S t e}+\frac{0.125}{S t e}\right)\left(0.8941-\frac{0.0244}{S t e}+0.6192 \frac{P k}{B^{\prime} i}\right)\left(1+\frac{\rho_{0} C_{p 0}\left(T_{a v e}-T_{c}\right)}{\Delta H^{*}}\right)
$$

Las cuatro ecuaciones fueron verificadas con datos experimentales, observándose que todas presentan el mismo rango de error. La principal desventaja de estas ecuaciones es que en todos los casos los parámetros fueron regresionados con medidas realizadas usando tilosa, y sólo se verificaron seis valores experimentales de muestras de carne picada.

\subsection{METODOS DE PREDICCION DESARROLLADOS}

\subsubsection{DESCONGELACION SIMETRICA}

El objetivo planteado al estudiar la descongelación es en un todo análogo al presentado en el inciso 2.4.1, de predicción de tiempos de congelación. 
Contando con los resultados que provee un modelo numérico, de diferencias finitas explícitas, suficientemente probado (Mascheroni, 1982), intentamos desarrollar una ecuación de predicción simpley precisa.

Con el modelo numérico se estudió un rango muy amplio de condiciones operativas, de modo de cubrir todos los valores que es posible hallar en la práctica industrial, que se detalla en la Tabla 3.3.

Tabla 3.3. Rango de variación de parámetros estudiado con el modelo numérico.

\begin{tabular}{||lccc||}
\hline Geometría & $\begin{array}{c}\mathrm{Ti} \\
\left({ }^{\circ} \mathrm{C}\right)\end{array}$ & $\begin{array}{c}\mathrm{Ta} \\
\left({ }^{\circ} \mathrm{C}\right)\end{array}$ & $\mathrm{Bi}$ \\
\hline \hline Placa plana $(\perp)$ & $-10 \mathrm{a}-31$ & $5 \mathrm{a} 35$ & 1 a 150 \\
Cilindro infinito & $-10 \mathrm{a}-35$ & $5 \mathrm{a} 45$ & 1 a 44 \\
Esfera & $-10 \mathrm{a}-35$ & $5 \mathrm{a} 45$ & $1 \mathrm{a} 44$ \\
\hline \hline
\end{tabular}

La Figura 3.1 nos muestra una serie de curvas de descongelación para el centro térmico, de placa plana infinita, obtenidas para distintas combinaciones de los parámetros $\mathrm{T}_{\mathrm{i}}, \mathrm{T}_{\mathrm{a}}$ y $\mathrm{Bi}$. 


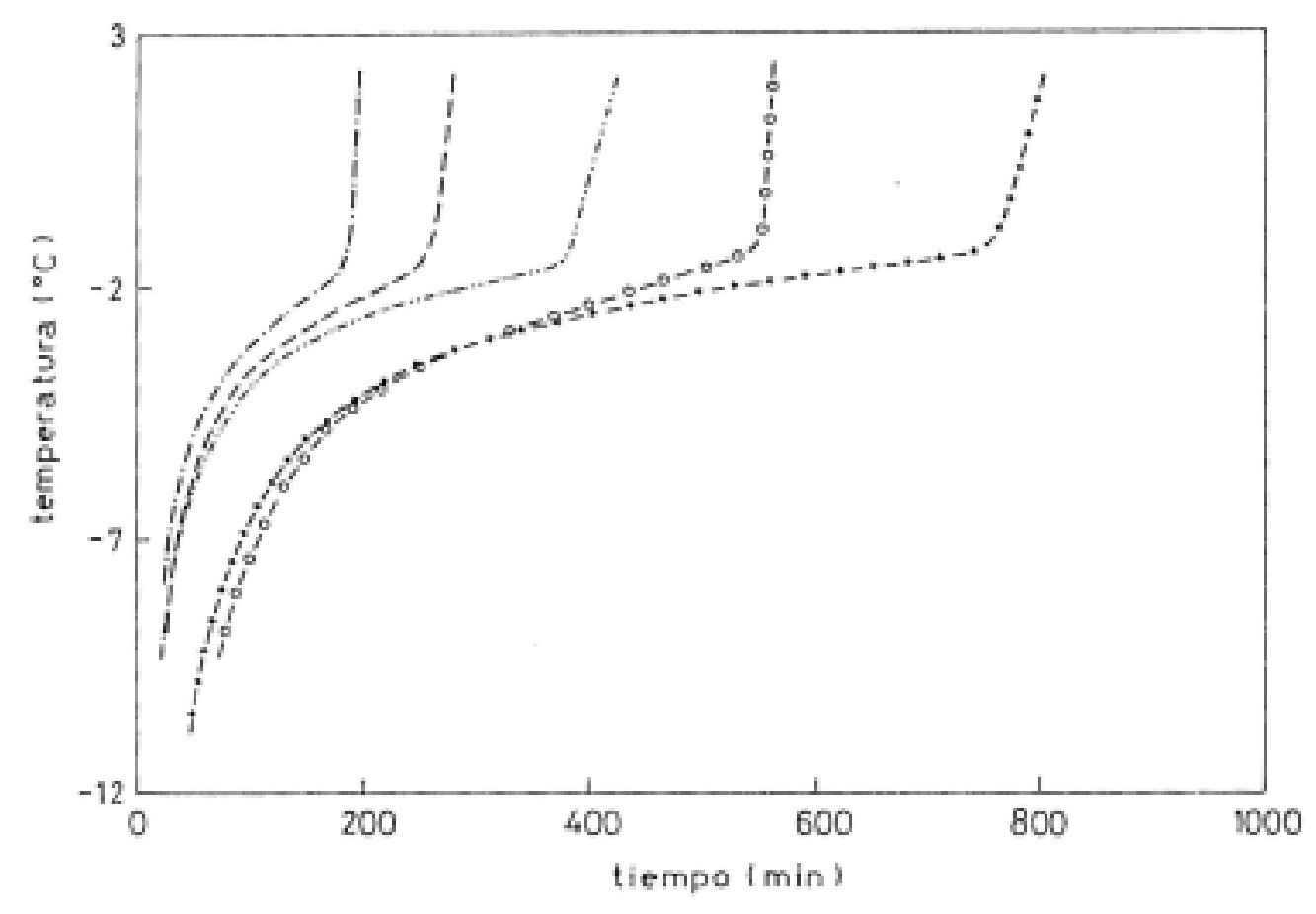

Figura 3.1. Curvas de descongelación para distintas condiciones operativas, para geometría plana.

A nálogamente a congelación, se observa que todas las curvas tienen la misma forma, si bien parten de distintos valores iniciales, en todas se presenta una zona de rápido aumento de la temperatura del centro luego que se produce la mayor parte del cambio de fase, en la región final de descongelación. Para formular un método aproximado de predicción del tiempo de descongelación tendríamos que encontrar una expresión que vincule la temperatura del centro térmico $T_{c}$ con el tiempo, considerando todas las variables involucradas en el proceso. Dicha expresión no necesariamente deberá valer para toda la curva de descongelación, sino que debe cumplirse en el tramo final, cuando la temperatura $T_{c}$ supera el valor inicial de cambio de fase y el producto se encuentra total mente descongelado.

Se propone probar la variable adimensional $X$ similar a la ya utilizada en los métodos de predicción de tiempos de congelación, buscando agrupar las distintas curvas de descongelación en una única curva representativa aún para los valores casos extremos de los parámetros indicados en la Tabla 3.3. Toda la complejidad del 
problema se vuelca en la definición de la variable X que deberá contemplar todos los parámetros del proceso además del tiempo t: temperatura del medio calefactor $T_{a}$, temperatura inicial $T_{i}$, coeficiente de transferencia calórica $h$, tamaño del producto $L$ (semiespesor en geometría plana o radio para cilindros y esferas) y propiedades físicas del alimento ( $\rho, C_{p}$ y $\left.k\right)$.

Después de probar distintas formas, basadas en resultados de los modelos teóricos aproximados, la variable X quedó definida por la siguiente ecuación:

$$
X=\frac{F o\left(\frac{T_{f}-T_{a}}{T_{f}}\right)^{m}}{\left(\frac{1}{B i}+c\right)\left(\frac{T_{i}-T_{f}}{T_{f}}\right)^{n}}
$$

Las propiedades físicas que aparecen en el número de Fourier (difusividad térmica $\alpha$ ) y en el número de Biot (conductividad térmica $k$ ) corresponden al estado fresco (sin congelar) ya que son más fáciles de obtener de la bibliografía, calcular o determinar experimentalmente.

Las constantes $\mathrm{c}, \mathrm{m}$ y $\mathrm{n}$ son constantes empíricas que dependen exclusivamente de la geometría, se presentan en la Tabla 3.4 .

Tabla 3.4. Constantes empíricas de la ec. (3.7).

\begin{tabular}{||lcrr||}
\hline \multicolumn{1}{|c}{ Geometría } & $\mathrm{c}$ & $\mathrm{m}$ & $\mathrm{n}$ \\
\hline \hline Placa plana & 0.45 & 0.74 & 0.03 \\
Cilindro infinito & 0.47 & 0.74 & 0.05 \\
Esfera & 0.45 & 0.715 & 0.03 \\
\hline
\end{tabular}

En la Figura 3.2 se han graficado las curvas de descongelación (sólo el tramo final) ya mostradas en la Figura 3.1, pero en función de X, para placa plana infinita. Las Figuras 3.3 y 3.4 son análogas a la Figura 3.2, pero corresponden a cilindro infinito y esfera, respectivamente. 


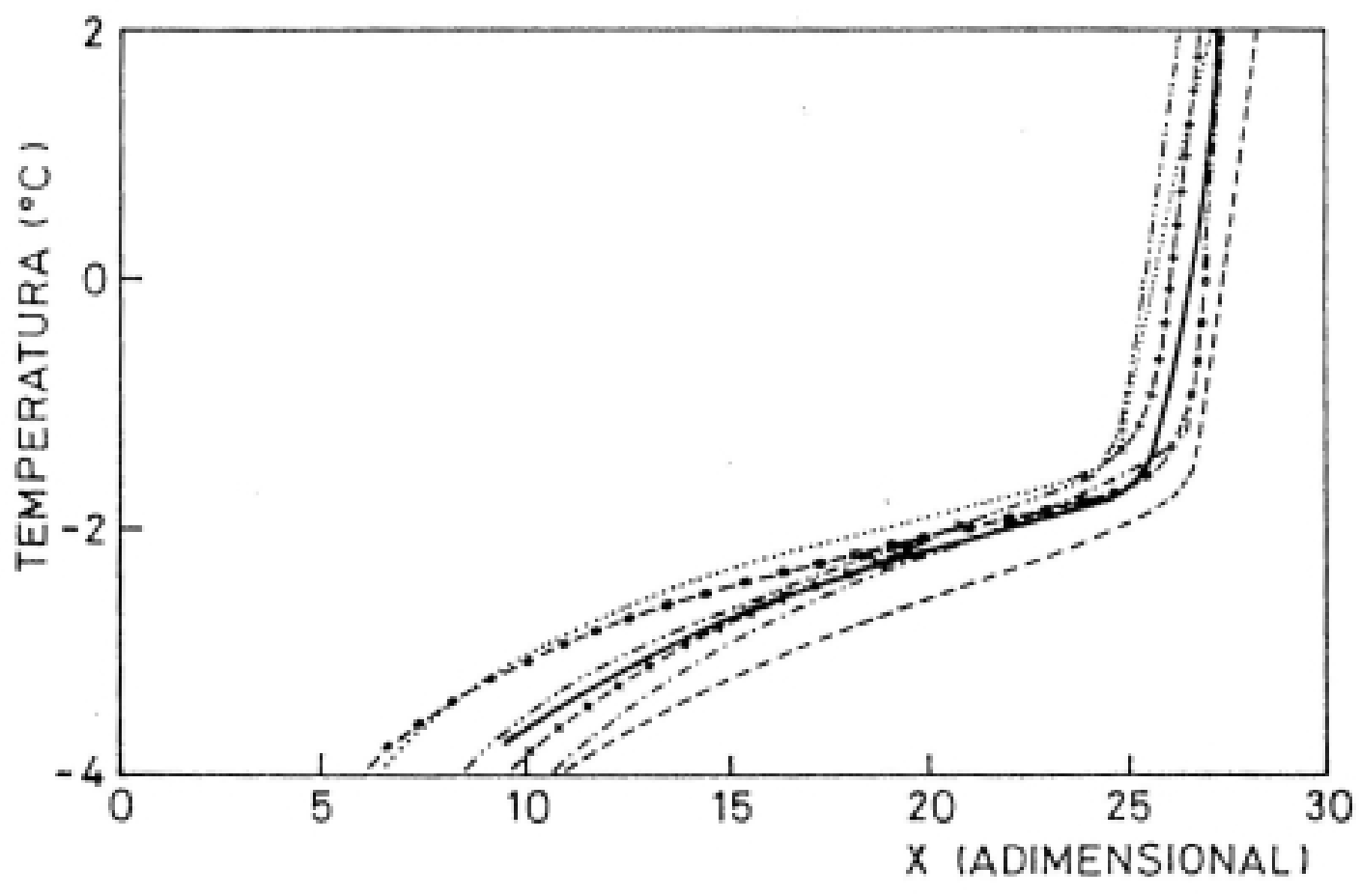

Figura 3.2. Temperatura del centro térmico en función de la variable adimensional $X$, descongelación de placa plana infinita.

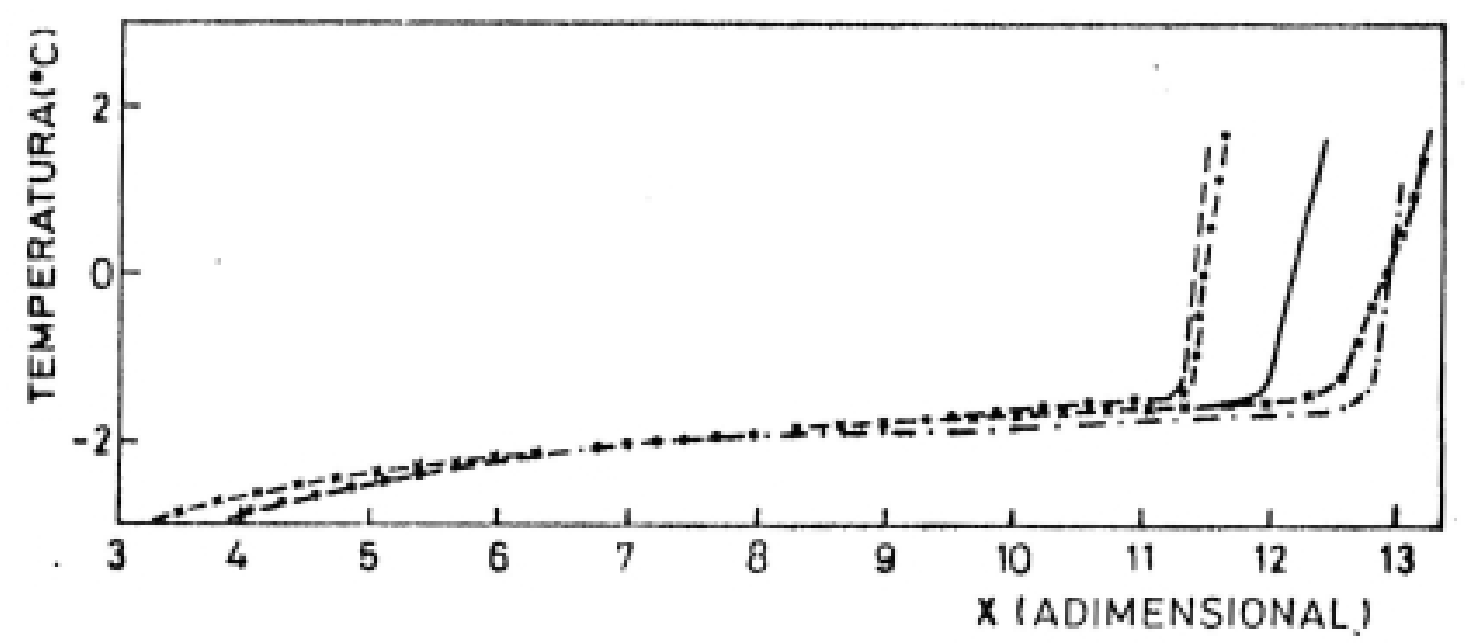

Figura 3.3. Temperatura del centro térmico en función de la variable adimensional $X$, descongelación de cilindro infinito. 


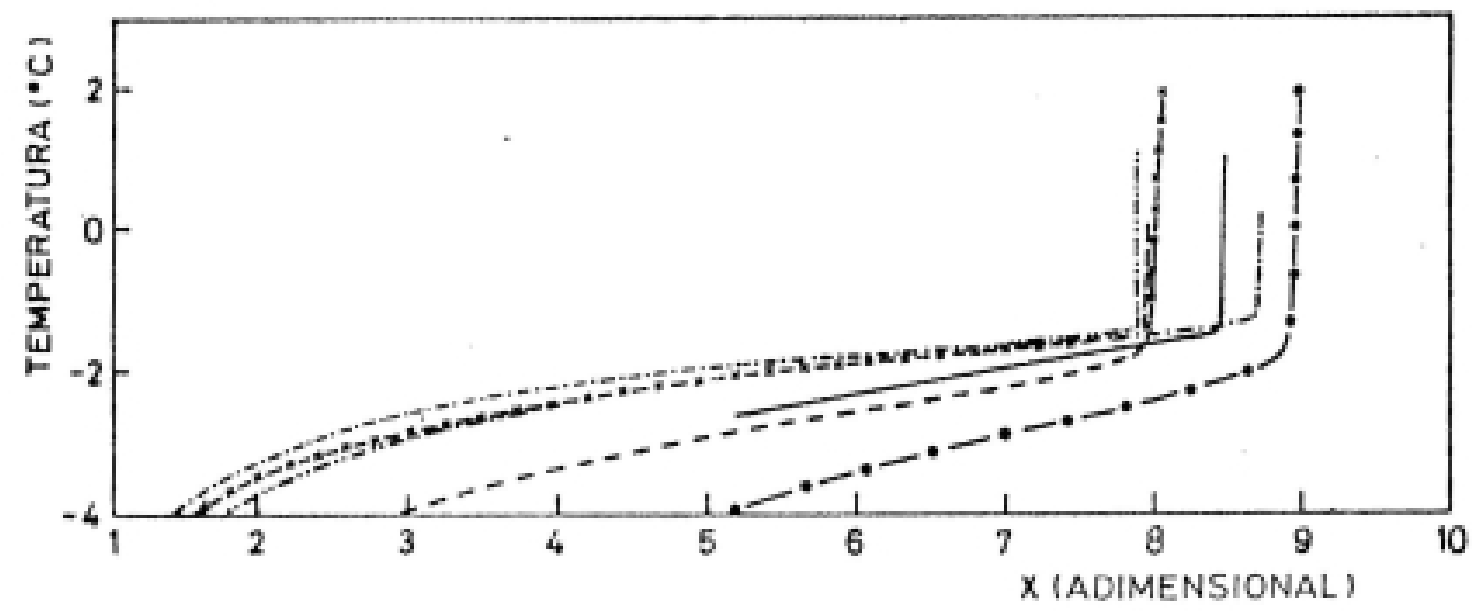

Figura 3.4. Temperatura del centro térmico en función de la variable adimensional $X$, descongelación de esfera.

En la ec. (3.7) aparece como variable la temperatura de comienzo de cambio de fase $T_{\text {f. }}$ Recordemos que el método fue desarrollado utilizando resultados numéricos obtenidos con propiedades de carne vacuna, para la cual Tf es aproximadamente $-1^{\circ} \mathrm{C}$ (varía un poco dependiendo del contenido inicial de agua Yo). Al utilizar este método con otros alimentos se comprobó que ajusta mejor la expresión siguiente, donde se ha reemplazado $\mathrm{Tf}_{\mathrm{f}}$ por $-1^{\circ} \mathrm{C}$ (en esta expresión debe trabajarse necesariamente con el valor de temperatura en grados Centígrados):

$$
X=\frac{F o\left(T_{a}+1\right)^{m}}{\left(\frac{1}{B i}+c\right)\left(-1-T_{i}\right)^{n}}
$$

El primer método de predicción propuesto se basaba en tomar de las Figuras el valor de X para una temperatura final determinada (de la línea llena promedio correspondiente, según la geometría). Luego, reordenando la ec. (3.8) y desadimensionalizando el tiempo (del número de Fourier, Fo) se calculaba el tiempo de descongelación td:

$$
t_{d}=X \frac{L^{2}}{\alpha_{0}}\left(\frac{1}{B i}+c\right)\left(-1-T_{i}\right)^{n}\left(T_{a}+1\right)^{-m}
$$


La desventaja que presenta esta metodología de cálculo reside en que es necesario utilizar gráficos para calcular $\mathrm{X}$ y además se incorpora un nuevo error (de lectura) en la predicción de td.

Tomando como base la ec. (3.8) que define la variable X, y admitiendo que la relación entre la temperatura del centro térmico, Tc y $X$ puede expresarse por una recta (ec. (2.54)) en la zona final de descongelación, se plantea una nueva ecuación de predicción, ec. (3.10) debiendo recalcular los parámetros empíricos anteriores (c, m y n) y los nuevos (a y b).

$$
t_{d}=\frac{L^{2}}{\alpha_{0}}\left(a T_{c}+b\right)\left(\frac{1}{B i}+c\right)\left(-1-T_{i}\right)^{n}\left(T_{a}+1\right)^{-m}
$$

Los parámetros fueron calculados regresionando el modelo propuesto con un paquete estadístico, Wilkinson (1990), con un método de regresión no lineal. Se presentan en la Tabla 3.5.

Tabla 3.5. Constantes empíricas de la ec. (3.10)

\begin{tabular}{|lrrrrr||}
\hline Geometría & $\mathrm{a}$ & $\mathrm{b}$ & $\mathrm{c}$ & $\mathrm{m}$ & \multicolumn{1}{c|}{$\mathrm{n}$} \\
\hline \hline Placa plana & 0.321 & 23.637 & 0.435 & 0.763 & 0.099 \\
infinita & $(0.044)$ & $(0.844)$ & $(0.007)$ & $(0.007)$ & $(0.010)$ \\
Cilindro & & & & & \\
infinito & 0.109 & 12.572 & 0.428 & 0.707 & 0.032 \\
& $(0.020)$ & $(0.127)$ & $(0.003)$ & $(0.004)$ & $(0.002)$ \\
Esfera & 0.039 & 8.120 & 0.408 & 0.671 & 0.027 \\
& $(0.017)$ & $(0.105)$ & $(0.004)$ & $(0.004)$ & $(0.002)$ \\
\hline
\end{tabular}

Nota: Los valores entre paréntesis corresponden a la desviación estándar de los parámetros estimados.

\subsubsection{RESULTADOS}

Nuevamente, para verificar la validez y precisión de la metodología de Cap. $3-13$ 
cálculo propuesta, se compararon los tiempos de descongelación experimentales de una variada gama de alimentos, abarcando todos los datos existentes en bibliografía, que detallaban completamente las condiciones en que se había efectuado la descongelación (forma y dimensiones del al imento, temperatura inicial, temperatura del medio cal efactor, coeficiente de transferencia calórica, temperatura final).

Los resultados se presentan en tablas, donde se describen las condiciones operativas, el tiempo de descongelación experimental y el tiempo predicho con la ec. (3.10). La difusividad térmica y la conductividad térmica del producto respectivo figuran en la Tabla 2.7. A nálogamente a lo hecho en congelación se utilizaron en algunos casos las propiedades presentadas por cada autor, en otros fue necesario buscar propiedades de bibliografía o utilizar al guna ecuación de predicción.

Las Tablas 3.6 a 3.17 corresponden a descongelación de alimentos asimilables a una placa plana con transferencia de energía en una única dirección. Se incluyen datos de geles, carnes, pescados y puré de papa.

Los mismos resultados se presentan en distintos gráficos, donde se han graficado los tiempos de descongelación calculados con la ec. (3.10) vs. los respectivos tiempos experimentales, la línea llena corresponde a la predicción exacta. En la Figura 3.5 ( $a$ y b) los datos se han diferenciado por tipo de producto, mientras que en la Figura 3.6 ( $a$ y b) la diferenciación se ha hecho en base a sus condiciones operativas, ya sea que caigan dentro o fuera del rango presentado en la Tabla 3.3, de estricta validez dela ecuación de cálculo.

Las Tablas 3.18 a 3.20 corresponden a alimentos asimilables a cilindro infinito, los mismos valores se han graficado en la Figura 3.7, diferenciados por tipo de producto y en la Figura 3.8 diferenciados por los valores de las condiciones operativas.

Las Tablas 3.21 a 3.23 corresponden a esferas, de tilosa, carne picada y puré de papa. A nálogamente a los casos anteriores, los resultados también se presentan en la Figura 3.9, diferenciados por tipo de producto y en la Figura 3.10 diferenciados por el valor de sus condiciones operativas. 
Tabla 3.6. Tiempos de descongel ación, experimentales y calculados. Placa plana, tilosa (Cleland y col.. 1986).

\begin{tabular}{|c|c|c|c|c|c|c|c|c|}
\hline $\begin{array}{c}\mathrm{L} \\
(\mathrm{cm})\end{array}$ & $\underset{W}{h} \stackrel{h}{\left(m^{2 \circ} \mathrm{C}\right.}$ & $\mathrm{Bi}$ & $\begin{array}{c}\mathrm{T}_{\mathrm{a}} \\
\left({ }^{\circ} \mathrm{C}\right)\end{array}$ & $\begin{array}{c}\mathrm{T}_{\mathrm{i}} \\
\left({ }^{\circ} \mathrm{C}\right)\end{array}$ & $\begin{array}{c}\mathrm{T}_{\mathrm{c}} \\
\left({ }^{\circ} \mathrm{C}\right)\end{array}$ & $\begin{array}{l}t_{d}, \exp \\
\text { (min) }\end{array}$ & $\begin{array}{l}t_{d}, p r e \\
\text { (min) }\end{array}$ & $\begin{array}{c}\mathrm{e} \\
(\%)\end{array}$ \\
\hline 1.30 & 13.2 & 0.31 & 12.8 & -20.9 & 0.0 & 281.4 & 293.2 & 4.20 \\
\hline 1.30 & 24.5 & 0.58 & 5.2 & -29.4 & 0.0 & 337.8 & 332.2 & -1.67 \\
\hline 1.30 & 24.5 & 0.58 & 45.9 & -8.3 & 0.0 & 54.0 & 62.0 & 14.82 \\
\hline 1.30 & 50.4 & 1.19 & 4.6 & -26.3 & 0.0 & 209.4 & 209.2 & -0.09 \\
\hline 1.30 & 78.1 & 1.85 & 5.2 & -12.3 & 0.0 & 150.6 & 137.0 & -9.04 \\
\hline 1.30 & 78.1 & 1.85 & 12.4 & -27.7 & 0.0 & 85.2 & 82.8 & -2.77 \\
\hline 2.63 & 24.5 & 1.17 & 5.2 & -10.7 & 0.0 & 792.0 & 726.7 & -8.24 \\
\hline 2.63 & 29.5 & 1.41 & 12.8 & -20.9 & 0.0 & 375.6 & 376.2 & 0.15 \\
\hline 2.63 & 50.4 & 2.41 & 5.1 & -25.0 & 0.0 & 564.6 & 530.7 & -6.01 \\
\hline 2.63 & 50.4 & 2.41 & 13.4 & -20.2 & 0.0 & 280.8 & 269.5 & -4.01 \\
\hline 2.63 & 50.4 & 2.41 & 13.4 & -24.1 & 0.0 & 279.0 & 274.5 & $-1.61 \|$ \\
\hline 2.63 & 50.4 & 2.41 & 13.4 & -23.6 & 0.0 & 276.0 & 273.9 & -0.75 \\
\hline 2.63 & 50.4 & 2.41 & 13.4 & -23.6 & 0.0 & 270.0 & 273.9 & 1.45 \\
\hline 2.63 & 78.1 & 3.73 & 5.2 & -28.9 & 0.0 & 454.8 & 439.8 & -3.30 \\
\hline 2.63 & 78.1 & 3.73 & 13.3 & -22.5 & 0.0 & 234.6 & 226.5 & -3.44 \\
\hline 2.63 & 13.2 & 0.63 & 46.1 & -28.6 & 0.0 & 233.4 & 268.9 & 15.21 \\
\hline 2.63 & 13.2 & 0.63 & 12.8 & -20.5 & 0.0 & 619.8 & 662.9 & 6.95 \\
\hline 2.63 & 13.2 & 0.63 & 5.2 & -11.4 & 0.0 & 1159.2 & 1147.0 & -1.06 \\
\hline 2.63 & 50.4 & 2.41 & 46.0 & -10.6 & 0.0 & 98.4 & 102.1 & 3.71 \\
\hline 3.85 & 18.2 & 1.27 & 46.2 & -26.8 & 0.0 & 306.6 & 346.0 & 12.87 \\
\hline 3.85 & 37.3 & 2.61 & 46.1 & -24.7 & 0.0 & 223.2 & 230.5 & 3.26 \\
\hline 3.85 & 50.4 & 3.53 & 45.7 & -13.5 & 0.0 & 179.4 & 191.2 & 6.60 \\
\hline 3.85 & 78.1 & 5.47 & 45.7 & -9.4 & 0.0 & 156.6 & 158.1 & 0.98 \\
\hline 3.85 & 37.3 & 2.61 & 5.2 & -30.2 & 0.0 & 1109.4 & 1105.4 & -0.37 \\
\hline 3.85 & 78.1 & 5.47 & 5.0 & -14.2 & 0.0 & 863.4 & 791.4 & -8.34 \\
\hline 3.85 & 78.1 & 5.47 & 12.9 & -21.0 & 0.0 & 435.0 & 434.4 & -0.14 \\
\hline 5.25 & 78.1 & 7.46 & 5.2 & -28.8 & 0.0 & 1417.2 & 1423.2 & 0.42 \\
\hline 5.25 & 172.7 & 16.49 & 13.4 & -23.8 & 0.0 & 636.6 & 638.9 & 0.37 \\
\hline 5.25 & 37.3 & 3.56 & 5.2 & -10.4 & 0.0 & 1759.8 & 1607.8 & -8.63 \\
\hline 1.40 & 172.7 & 4.40 & 43.0 & -31.0 & 0.0 & 25.8 & 26.6 & 3.14 \\
\hline 5.10 & 13.2 & 1.22 & 43.0 & -13.7 & 0.0 & 529.8 & 612.9 & 15.69 \\
\hline 5.00 & 78.1 & 7.10 & 46.2 & -28.2 & 0.0 & 275.4 & 276.9 & 0.56 \\
\hline 5.00 & 13.2 & 1.20 & 45.9 & -8.3 & 0.0 & 469.2 & 538.1 & 14.69 \\
\hline 5.00 & 24.5 & 2.23 & 45.8 & -32.5 & 0.0 & 405.0 & 434.2 & 7.20 \\
\hline 5.00 & 24.5 & 2.23 & 12.8 & -9.4 & 0.0 & 1015.2 & 967.2 & -4.73 \\
\hline
\end{tabular}

Tabla 3.7. Tiempos de descongelación, experimentales y calculados. Placa plana, gel de agar (Flores y col., 1993).

\begin{tabular}{|c|c|c|c|c|c|c|c|c|}
\hline$\underset{(\mathrm{cm})}{\mathrm{L}}$ & $\begin{array}{c}\mathrm{h} \\
\mathrm{W} /\left(\mathrm{m}^{2 \circ} \mathrm{C}\right. \\
)\end{array}$ & $\mathrm{Bi}$ & $\begin{array}{c}\mathrm{T}_{\mathrm{a}} \\
\left({ }^{\circ} \mathrm{C}\right)\end{array}$ & $\begin{array}{c}\mathrm{T}_{\mathrm{i}} \\
\left({ }^{\circ} \mathrm{C}\right)\end{array}$ & $\begin{array}{c}\mathrm{T}_{\mathrm{c}} \\
\left({ }^{\circ} \mathrm{C}\right)\end{array}$ & $\begin{array}{l}t_{d}, \exp \\
(\min )\end{array}$ & $\begin{array}{l}\mathrm{t}_{\mathrm{d}, \mathrm{pre}} \\
\text { (min) }\end{array}$ & $\begin{array}{c}\mathrm{e} \\
(\%)\end{array}$ \\
\hline
\end{tabular}


Tiempos de descongelación

\begin{tabular}{|c|c|c|c|c|c|c|c|c|}
\hline $\begin{array}{c}\mathrm{L} \\
(\mathrm{cm})\end{array}$ & $\begin{array}{c}h \\
\mathrm{~W} /\left(\mathrm{m}^{2 \circ} \mathrm{C}\right. \\
\mathrm{r} \\
\end{array}$ & $\mathrm{Bi}$ & $\begin{array}{c}\mathrm{Ta}_{\mathrm{a}} \\
\left({ }^{\circ} \mathrm{C}\right)\end{array}$ & $\begin{array}{c}\mathrm{T}_{\mathrm{i}} \\
\left({ }^{\circ} \mathrm{C}\right)\end{array}$ & $\begin{array}{c}\mathrm{T}_{\mathrm{c}} \\
\left({ }^{\circ} \mathrm{C}\right)\end{array}$ & $\begin{array}{l}t_{d,}, \exp \\
\text { (min) }\end{array}$ & $\begin{array}{l}t_{d,}, p r e \\
\text { (min) }\end{array}$ & $\begin{array}{c}\mathrm{e} \\
(\%)\end{array}$ \\
\hline 3.00 & 150.0 & 7.98 & 10.0 & -16.0 & 0.0 & 330.0 & 311.1 & -5.74 \\
\hline 3.00 & 150.0 & 7.98 & 10.0 & -14.0 & 0.0 & 300.0 & 306.7 & 2.23 \\
\hline 3.00 & 150.0 & 7.98 & 15.0 & -11.0 & 0.0 & 240.0 & 224.5 & -6.45 \\
\hline 3.00 & 300.0 & 15.96 & 18.0 & -10.5 & 0.0 & 190.2 & 174.0 & -8.50 \\
\hline 4.00 & 150.0 & 10.64 & 10.0 & -15.0 & 0.0 & 555.0 & 518.5 & -6.57 \\
\hline 4.00 & 150.0 & 10.64 & 10.0 & -12.8 & 0.0 & 520.2 & 509.8 & -1.99 \\
\hline 4.00 & 600.0 & 42.55 & 18.0 & -15.0 & 0.0 & 330.0 & 296.2 & -10.24 \\
\hline 4.00 & 600.0 & 42.55 & 15.0 & -12.0 & 0.0 & 360.0 & 329.7 & -8.41 \\
\hline 4.00 & 300.0 & 21.28 & 15.0 & -10.0 & 0.0 & 360.0 & 339.8 & -5.61 \\
\hline 4.00 & 600.0 & 42.55 & 20.0 & -16.0 & 0.0 & 289.8 & 276.3 & -4.66 \\
\hline 4.00 & 150.0 & 10.64 & 20.0 & -15.0 & 0.0 & 330.0 & 316.6 & -4.06 \\
\hline
\end{tabular}

Tabla 3.8. Tiempos de descongel ación, experimentales y calculados. Placa plana, carne (James y col., 1977).

\begin{tabular}{|c|c|c|c|c|c|c|c|c|}
\hline $\begin{array}{c}\mathrm{L} \\
(\mathrm{cm})\end{array}$ & $\begin{array}{c}h \\
\mathrm{~W} /\left(\mathrm{m}^{2 \circ} \mathrm{C}\right. \\
) \\
\end{array}$ & $\mathrm{Bi}$ & $\begin{array}{c}\mathrm{T}_{\mathrm{a}} \\
\left({ }^{\circ} \mathrm{C}\right)\end{array}$ & $\begin{array}{c}\mathrm{T}_{\mathrm{i}} \\
\left({ }^{\circ} \mathrm{C}\right)\end{array}$ & $\begin{array}{c}\mathrm{T}_{\mathrm{c}} \\
\left({ }^{\circ} \mathrm{C}\right)\end{array}$ & $\begin{array}{l}t_{d}, \exp \\
(\min )\end{array}$ & $\begin{array}{l}t_{d}, p r e \\
\text { (min) }\end{array}$ & $\begin{array}{c}e \\
(\%)\end{array}$ \\
\hline 5.00 & 25.0 & 2.60 & 10.0 & -30.0 & 0.0 & 1470.0 & 1379.1 & -6.19 \\
\hline 5.00 & 25.0 & 2.60 & 10.0 & -30.0 & 0.0 & 1380.0 & 1379.1 & -0.07 \\
\hline 5.00 & 25.0 & 2.60 & 10.0 & -28.0 & 0.0 & 1320.0 & 1369.4 & 3.74 \\
\hline 5.00 & 25.0 & 2.60 & 10.0 & -26.0 & 0.0 & 1320.0 & 1359.0 & 2.95 \\
\hline
\end{tabular}

Tabla 3.9. Tiempos de descongel ación, experimentales y calculados. Placa plana, carne deshuesada (James \& Bailey, 1980).

\begin{tabular}{|c|c|c|c|c|c|c|c|c|}
\hline $\begin{array}{c}\mathrm{L} \\
(\mathrm{cm})\end{array}$ & $\begin{array}{c}h \\
\mathrm{~W} /\left(\mathrm{m}^{2 \circ} \mathrm{C}\right. \\
)\end{array}$ & $\mathrm{Bi}$ & $\begin{array}{c}\mathrm{T}_{\mathrm{a}} \\
\left({ }^{\circ} \mathrm{C}\right)\end{array}$ & $\begin{array}{c}\mathrm{T}_{\mathrm{i}} \\
\left({ }^{\circ} \mathrm{C}\right)\end{array}$ & $\begin{array}{c}\mathrm{T}_{\mathrm{c}} \\
\left({ }^{\circ} \mathrm{C}\right)\end{array}$ & $\begin{array}{l}t_{d}, \exp \\
(\min )\end{array}$ & $\begin{array}{l}t_{d,}, p r e \\
\text { (min) }\end{array}$ & $\begin{array}{c}\mathrm{e} \\
(\%)\end{array}$ \\
\hline 6.75 & 15.0 & 2.11 & 10.9 & -30.0 & 0.0 & 2532.0 & 2627.3 & 3.76 \\
\hline 6.75 & 15.0 & 2.11 & 19.3 & -30.0 & 0.0 & 1596.0 & 1748.0 & 9.52 \\
\hline 6.75 & 15.0 & 2.11 & 29.1 & -30.0 & 0.0 & 990.0 & 1294.2 & 30.73 \\
\hline 6.75 & 40.0 & 5.63 & 10.3 & -30.0 & 0.0 & 1896.0 & 1842.3 & -2.83 \\
\hline 6.75 & 40.0 & 5.63 & 19.7 & -30.0 & 0.0 & 1146.0 & 1160.8 & 1.29 \\
\hline 6.75 & 40.0 & 5.63 & 20.9 & -30.0 & $0.0 \|$ & 876.0 & 1112.0 & 26.94 \\
\hline 6.75 & 1000.0 & 140.63 & 10.0 & -30.0 & 0.0 & 1143.0 & 1356.8 & 18.70 \\
\hline 6.75 & 1000.0 & 140.63 & 14.3 & -30.0 & 0.0 & 921.0 & 1054.8 & 14.53 \\
\hline 6.75 & 1000.0 & 140.63 & 20.0 & -30.0 & 0.0 & 837.0 & 828.4 & -1.03 \\
\hline 6.75 & 1000.0 & 140.63 & 25.0 & -30.0 & 0.0 & 615.0 & 703.8 & 14.44 \\
\hline 6.75 & 1000.0 & 140.63 & 30.0 & -30.0 & 0.0 & 582.0 & 615.4 & 5.74 \\
\hline
\end{tabular}

Tabla 3.10. Tiempos de descongelación, experimentales y calculados. Placa plana, cordero (Flores y col., 1993). 
Tiempos de descongelación

\begin{tabular}{|c|c|c|c|c|c|c|c|c|}
\hline $\begin{array}{c}\mathrm{L} \\
(\mathrm{cm})\end{array}$ & $\begin{array}{c}h \\
\mathrm{~W} /\left(\mathrm{m}^{2 \circ} \mathrm{C}\right. \\
)\end{array}$ & $\mathrm{Bi}$ & $\begin{array}{c}\mathrm{Ta} \\
\left({ }^{\circ} \mathrm{C}\right)\end{array}$ & $\begin{array}{c}\mathrm{T}_{\mathrm{i}} \\
\left({ }^{\circ} \mathrm{C}\right)\end{array}$ & $\begin{array}{c}\mathrm{T}_{\mathrm{c}} \\
\left({ }^{\circ} \mathrm{C}\right)\end{array}$ & $\begin{array}{l}t_{d}, \exp \\
(\min )\end{array}$ & $\begin{array}{l}\text { td,pre } \\
\text { (min) }\end{array}$ & $\begin{array}{c}\mathrm{e} \\
(\%)\end{array}$ \\
\hline 3.00 & 18.8 & 1.14 & 34.8 & -27.0 & 0.0 & 240.0 & 296.0 & 23.32 \\
\hline 3.00 & 16.7 & 1.01 & 29.8 & -16.2 & 0.0 & 312.0 & 339.7 & 8.89 \\
\hline 3.00 & 15.8 & 0.96 & 34.5 & -14.8 & 0.0 & 279.0 & 313.8 & 12.49 \\
\hline 3.00 & 28.8 & 1.75 & 30.9 & -13.9 & 0.0 & 253.8 & 231.2 & -8.92 \\
\hline 5.00 & 51.9 & 5.24 & 30.5 & -29.1 & 0.0 & 365.4 & 434.8 & 19.00 \\
\hline 5.00 & 32.3 & 3.26 & 29.0 & -27.5 & 0.0 & 453.0 & 531.2 & 17.25 \\
\hline 5.00 & 38.9 & 3.93 & 20.3 & -26.6 & 0.0 & 569.4 & 640.2 & 12.43 \\
\hline 5.00 & 22.7 & 2.29 & 34.1 & -26.0 & 0.0 & 497.4 & 550.9 & 10.75 \\
\hline 5.00 & 39.2 & 3.96 & 9.9 & -18.5 & 0.0 & 941.4 & 1022.9 & 8.65 \\
\hline 5.00 & 46.4 & 4.69 & 35.2 & -16.2 & 0.0 & 360.0 & 381.0 & 5.84 \\
\hline 5.00 & 32.9 & 3.32 & 30.4 & -15.3 & 0.0 & 471.0 & 478.8 & 1.65 \\
\hline 5.00 & 42.5 & 4.29 & 27.2 & -15.0 & 0.0 & 408.0 & 471.5 & 15.56 \\
\hline
\end{tabular}

Tabla 3.11. Tiempos de descongelación, experimentales y calcu-lados. Placa plana, carne picada, $Y_{0}=0.74$ (Cleland y col., 1986).

\begin{tabular}{|c|c|c|c|c|c|c|c|c|}
\hline $\begin{array}{c}\mathrm{L} \\
(\mathrm{cm})\end{array}$ & $\underset{W}{h} \stackrel{h}{\left(m^{2 \circ} \mathrm{C}\right.}$ & $\mathrm{Bi}$ & $\begin{array}{c}\mathrm{T}_{\mathrm{a}} \\
\left({ }^{\circ} \mathrm{C}\right)\end{array}$ & $\begin{array}{c}\mathrm{T}_{\mathrm{i}} \\
\left({ }^{\circ} \mathrm{C}\right)\end{array}$ & $\begin{array}{c}\mathrm{T}_{\mathrm{c}} \\
\left({ }^{\circ} \mathrm{C}\right)\end{array}$ & $\begin{array}{l}t_{d}, \exp \\
\text { (min) }\end{array}$ & $\begin{array}{l}\text { td,pre } \\
\text { (min) }\end{array}$ & $\begin{array}{c}\mathrm{e} \\
(\%)\end{array}$ \\
\hline 1.20 & 13.2 & 0.32 & 5.8 & -19.1 & 0.0 & 514.8 & 458.9 & -10.87 \\
\hline 1.20 & 50.4 & 1.21 & 26.9 & -15.6 & 0.0 & 50.4 & 53.7 & 6.63 \\
\hline 2.35 & 18.2 & 0.86 & 8.0 & -27.1 & 0.0 & 724.2 & 658.0 & -9.14 \\
\hline 2.35 & 78.1 & 3.67 & 15.8 & -24.4 & 0.0 & 171.0 & 178.3 & 4.28 \\
\hline 3.75 & 24.5 & 1.84 & 43.2 & -29.0 & 0.0 & 312.6 & 305.8 & -2.17 \\
\hline 3.75 & 172.7 & 12.95 & 9.3 & -16.9 & 0.0 & 536.4 & 459.6 & -14.32 \\
\hline
\end{tabular}

Tabla 3.12. Tiempos de descongelación, experimentales y calculados. Placa plana, carne picada, $Y_{0}=0.68$ (Ilicali, 1989).

\begin{tabular}{|c|c|c|c|c|c|c|c|c|}
\hline $\begin{array}{c}\mathrm{L} \\
(\mathrm{cm})\end{array}$ & $\begin{array}{c}h \\
W /\left(m^{2 \circ} \mathrm{C}\right.\end{array}$ & $\mathrm{Bi}$ & $\begin{array}{c}\mathrm{T}_{\mathrm{a}} \\
\left({ }^{\circ} \mathrm{C}\right)\end{array}$ & $\begin{array}{c}\mathrm{Ti}_{\mathrm{i}} \\
\left({ }^{\circ} \mathrm{C}\right)\end{array}$ & $\begin{array}{c}\mathrm{T}_{\mathrm{c}} \\
\left({ }^{\circ} \mathrm{C}\right)\end{array}$ & $\begin{array}{l}\mathrm{td}_{\mathrm{d}} \exp \\
(\min )\end{array}$ & $\begin{array}{l}\text { td,pre } \\
\text { (min) }\end{array}$ & $\begin{array}{c}e \\
(\%)\end{array}$ \\
\hline 2.00 & 16.2 & 0.75 & 24.8 & -29.8 & 5.0 & 263.0 & 266.2 & 1.23 \\
\hline 2.00 & 16.2 & 0.75 & 26.8 & -30.6 & 5.0 & 239.0 & 252.2 & 5.51 \\
\hline 2.00 & 44.6 & 2.06 & 27.8 & -37.0 & 5.0 & 131.0 & 130.0 & -0.79 \\
\hline 2.00 & 44.6 & 2.06 & 29.8 & -33.2 & 5.0 & 125.5 & 122.1 & -2.69 \\
\hline 2.00 & 61.4 & 2.83 & 31.0 & -31.2 & 5.0 & 104.5 & 100.8 & -3.51 \\
\hline 2.00 & 61.4 & 2.83 & 27.0 & -13.0 & 5.0 & 92.0 & 101.9 & 10.76 \\
\hline
\end{tabular}

Cap. $3-17$ 
Tiempos de descongelación

Tabla 3.13. Tiempos de descongelación, experimentales y cal culados. Placa plana, carne picada, $Y_{0}=0.68$ (Flores y col., 1993).

\begin{tabular}{|c|c|c|c|c|c|c|c|c|}
\hline$\underset{(\mathrm{cm})}{\mathrm{L}}$ & $\begin{array}{c}\mathrm{h} \\
\mathrm{W} /\left(\mathrm{m}^{2 \circ} \mathrm{C}\right. \\
)\end{array}$ & $\mathrm{Bi}$ & $\begin{array}{c}\mathrm{T}_{\mathrm{a}} \\
\left({ }^{\circ} \mathrm{C}\right)\end{array}$ & $\begin{array}{c}\mathrm{T}_{\mathrm{i}} \\
\left({ }^{\circ} \mathrm{C}\right)\end{array}$ & $\begin{array}{c}\mathrm{T}_{\mathrm{c}} \\
\left({ }^{\circ} \mathrm{C}\right)\end{array}$ & $\begin{array}{l}t_{d}, \exp \\
(\min )\end{array}$ & $\begin{array}{l}t_{d}, \text { pre } \\
\text { (min) }\end{array}$ & $\begin{array}{c}\mathrm{e} \\
(\%)\end{array}$ \\
\hline 3.00 & 150.0 & 10.37 & 15.0 & -15.8 & 0.0 & 220.2 & 226.5 & 2.84 \\
\hline 4.00 & 150.0 & 13.82 & 10.0 & -19.6 & 0.0 & 510.0 & 523.2 & 2.59 \\
\hline 4.00 & 300.0 & 27.65 & 10.0 & -19.4 & 0.0 & 495.0 & 485.4 & -1.94 \\
\hline
\end{tabular}

Tabla 3.14. Tiempos de descongelación, experimentales y calculados. Placa plana, bacalao (Vanichseni, 1971).

\begin{tabular}{||cccccc|cc|c||}
\hline \begin{tabular}{c}
\hline $\mathrm{L}$ \\
$(\mathrm{cm})$
\end{tabular} & $\begin{array}{c}\mathrm{h} \\
\mathrm{W} /\left(\mathrm{m}^{2 \circ} \mathrm{C}\right. \\
)\end{array}$ & $\mathrm{Bi}$ & $\begin{array}{c}\mathrm{T}_{\mathrm{a}} \\
\left({ }^{\circ} \mathrm{C}\right)\end{array}$ & $\begin{array}{c}\mathrm{T}_{\mathrm{i}} \\
\left({ }^{\circ} \mathrm{C}\right)\end{array}$ & $\begin{array}{c}\mathrm{T}_{\mathrm{c}} \\
\left({ }^{\circ} \mathrm{C}\right)\end{array}$ & $\begin{array}{c}\mathrm{t}_{d}, \exp \\
(\min )\end{array}$ & $\begin{array}{c}\mathrm{t}_{d}, \mathrm{pre} \\
(\mathrm{min})\end{array}$ & $\begin{array}{c}\mathrm{e} \\
(\%)\end{array}$ \\
\hline \hline 5.00 & 14.0 & 1.49 & 15.0 & -18.0 & 0.0 & 1369.2 & 1380.4 & 0.82 \\
\hline \hline
\end{tabular}

Tabla 3.15. Tiempos de descongelación, experimentales y calculados. Placa plana, sardina (Crepey \& Becel, 1978).

\begin{tabular}{|c|c|c|c|c|c|c|c|c|}
\hline $\begin{array}{c}\mathrm{L} \\
(\mathrm{cm})\end{array}$ & $\begin{array}{c}h \\
\mathrm{~W} / \mathrm{m}^{2 \circ} \mathrm{C}\end{array}$ & $\mathrm{Bi}$ & $\begin{array}{c}\mathrm{T}_{\mathrm{a}} \\
\left({ }^{\circ} \mathrm{C}\right)\end{array}$ & $\begin{array}{c}\mathrm{T}_{\mathrm{i}} \\
\left({ }^{\circ} \mathrm{C}\right)\end{array}$ & $\begin{array}{c}\mathrm{T}_{\mathrm{c}} \\
\left({ }^{\circ} \mathrm{C}\right)\end{array}$ & $\begin{array}{l}t_{d}, \exp \\
(\min )\end{array}$ & $\begin{array}{l}\text { td,pre } \\
\text { (min) }\end{array}$ & $\begin{array}{c}\mathrm{e} \\
(\%)\end{array}$ \\
\hline 3.25 & 8.0 & 0.56 & 4.5 & -20.0 & 0.0 & 2055.0 & 1923.7 & -6.39 \\
\hline 3.25 & 8.0 & 0.56 & 20.5 & -20.0 & 0.0 & 810.0 & 679.8 & -16.07 \\
\hline 3.25 & 1000.0 & 70.04 & 14.5 & -20.0 & 0.0 & 153.0 & 176.6 & 15.44 \\
\hline 3.25 & 600.0 & 42.03 & 15.0 & -20.0 & 0.0 & 178.8 & 176.1 & -1.54 \\
\hline
\end{tabular}

Tabla 3.16. Tiempos de descongelación, experimentales y calculados. Placa plana, caballa (Flechtenmacher, 1983).

\begin{tabular}{|c|c|c|c|c|c|c|c|c|}
\hline $\begin{array}{c}\mathrm{L} \\
(\mathrm{cm})\end{array}$ & $\begin{array}{c}h \\
\mathrm{~W} / \mathrm{m}^{2 \circ} \mathrm{C}\end{array}$ & $\mathrm{Bi}$ & $\begin{array}{c}\mathrm{T}_{\mathrm{a}} \\
\left({ }^{\circ} \mathrm{C}\right)\end{array}$ & $\begin{array}{c}\mathrm{T}_{\mathrm{i}} \\
\left({ }^{\circ} \mathrm{C}\right)\end{array}$ & $\begin{array}{c}\mathrm{Tc}_{\mathrm{c}} \\
\left({ }^{\circ} \mathrm{C}\right)\end{array}$ & $\begin{array}{l}t_{d}, \exp \\
\text { (min) }\end{array}$ & $\begin{array}{l}\text { td,pre } \\
\text { (min) }\end{array}$ & $\begin{array}{c}\mathrm{e} \\
(\%)\end{array}$ \\
\hline 3.30 & 300.0 & 21.38 & 18.3 & -28.9 & 0.0 & 223.2 & 225.8 & 1.16 \\
\hline 3.25 & 300.0 & 21.06 & 19.1 & -28.3 & 0.0 & 195.0 & 212.2 & 8.81 \\
\hline 3.25 & 300.0 & 21.06 & 19.1 & -30.8 & 0.0 & 208.2 & 214.0 & 2.79 \\
\hline 3.00 & 300.0 & 19.44 & 19.5 & -30.6 & 0.0 & 205.8 & 181.0 & -12.06 \\
\hline 3.35 & 300.0 & 21.71 & 19.8 & -30.6 & 0.0 & 196.8 & 220.7 & 12.16 \\
\hline
\end{tabular}


Tabla 3.17. Tiempos de descongelación, experimentales y calculados. Placa plana, puré de papa (Ilicali, 1989).

\begin{tabular}{|c|c|c|c|c|c|c|c|c|}
\hline $\begin{array}{c}\mathrm{L} \\
(\mathrm{cm})\end{array}$ & $\begin{array}{c}\mathrm{h} \\
\mathrm{W} /\left(\mathrm{m}^{2 \circ} \mathrm{C}\right. \\
) \\
\end{array}$ & $\mathrm{Bi}$ & $\begin{array}{c}\mathrm{T}_{\mathrm{a}} \\
\left({ }^{\circ} \mathrm{C}\right)\end{array}$ & $\begin{array}{c}\mathrm{T}_{\mathrm{i}} \\
\left({ }^{\circ} \mathrm{C}\right)\end{array}$ & $\begin{array}{c}\mathrm{T}_{\mathrm{c}} \\
\left({ }^{\circ} \mathrm{C}\right)\end{array}$ & $\begin{array}{l}t_{d}, \exp \\
(\min )\end{array}$ & $\begin{array}{l}t_{d}, \text { pre } \\
\text { (min) }\end{array}$ & $\begin{array}{c}\mathrm{e} \\
(\%)\end{array}$ \\
\hline 2.00 & 16.2 & 0.61 & 27.8 & -31.8 & 5.0 & 297.0 & 259.8 & -12.52 \\
\hline 2.00 & 16.2 & 0.61 & 31.6 & -21.6 & 5.0 & 230.5 & 227.1 & -1.46 \\
\hline 2.00 & 44.6 & 1.68 & 26.1 & -16.2 & 5.0 & 130.5 & 126.1 & -3.35 \\
\hline 2.00 & 44.6 & 1.68 & 25.7 & -11.6 & 5.0 & 132.5 & 123.1 & -7.10 \\
\hline 2.00 & 61.4 & 2.32 & 30.2 & -29.6 & 5.0 & 102.0 & 101.5 & -0.45 \\
\hline 2.00 & 61.4 & 2.32 & 28.4 & -23.2 & 5.0 & 116.5 & 103.6 & -11.06 \\
\hline
\end{tabular}

350

300

250

$$
++\sqrt{4 \text { 类 }} \times
$$

200

$+\quad \times+\square+$

150

100

50

0

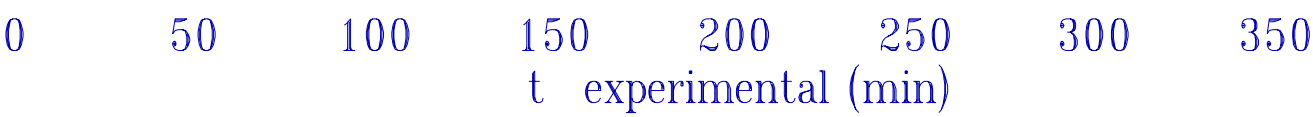

$\square$ Geles $\times$ Carnes + Pescados $\stackrel{\text { 全 }}{\text { Pure de papa }}$

Figura 3.5a. Tiempos de descongelación calculados vs. experimentales, placa plana, diferenciados por tipo de producto. 
2500

3200

1900

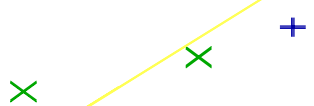

1600

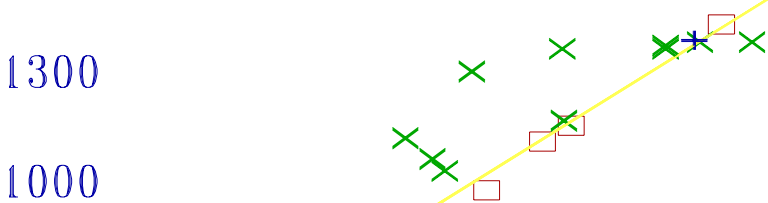

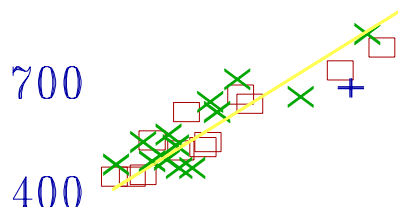

$\begin{array}{llllllll}400 & 700 & 1000 & 1300 & 1600 & 1900 & 2200 & 2500\end{array}$ experimental (min)

Geles $\times$ Carres + Pescados

Figura 3.5b. Tiempos de descongelación calculados vs. experimentales, placa plana, diferenciados por tipo de producto.

Cap. $3-20$ 


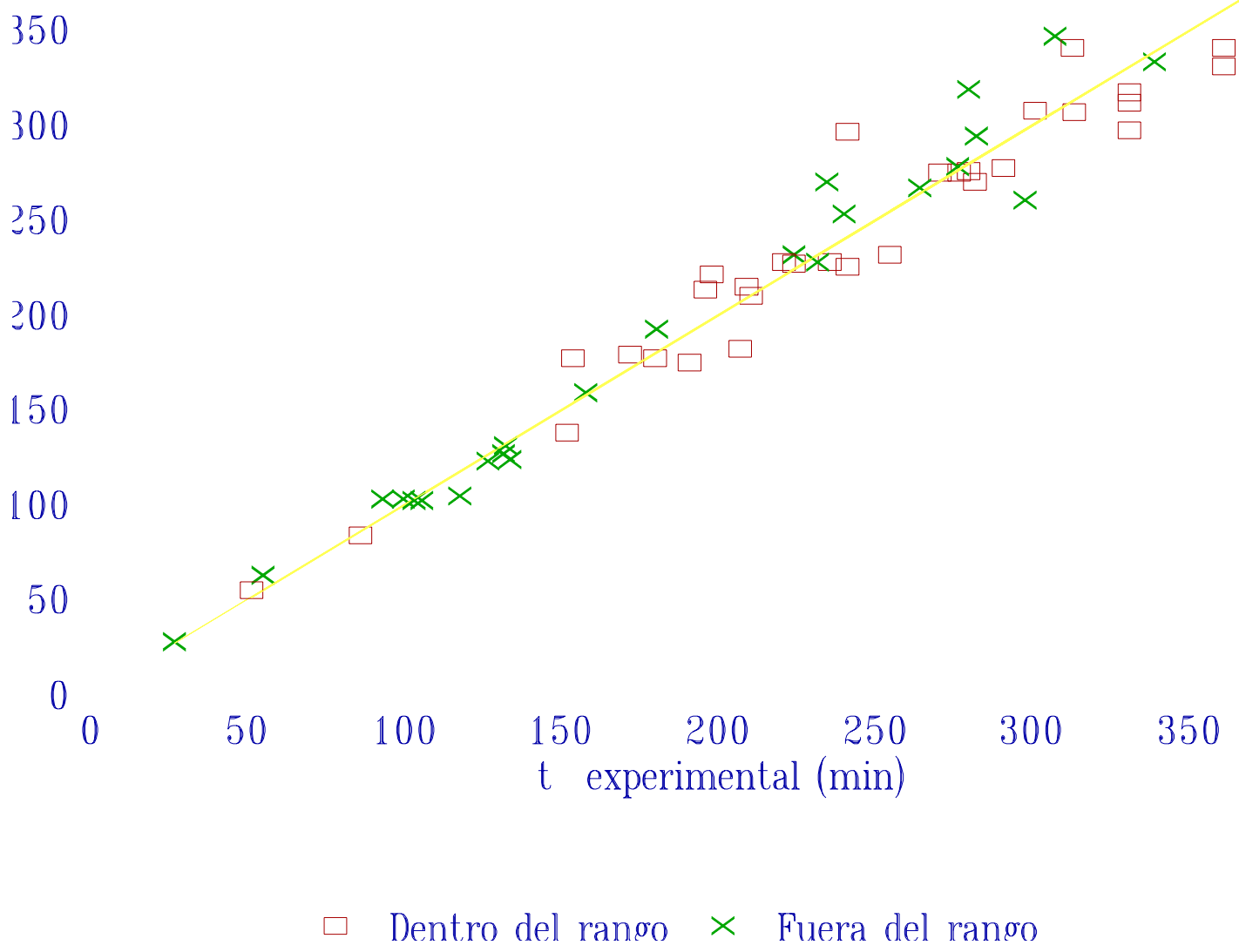

Figura 3.6a. Tiempos de descongelación calculados vs. experimentales, placa plana, diferenciados según sus condiciones operativas estén dentro o fuera del rango de estricta validez del método de predicción.

Cap. 3 -21 


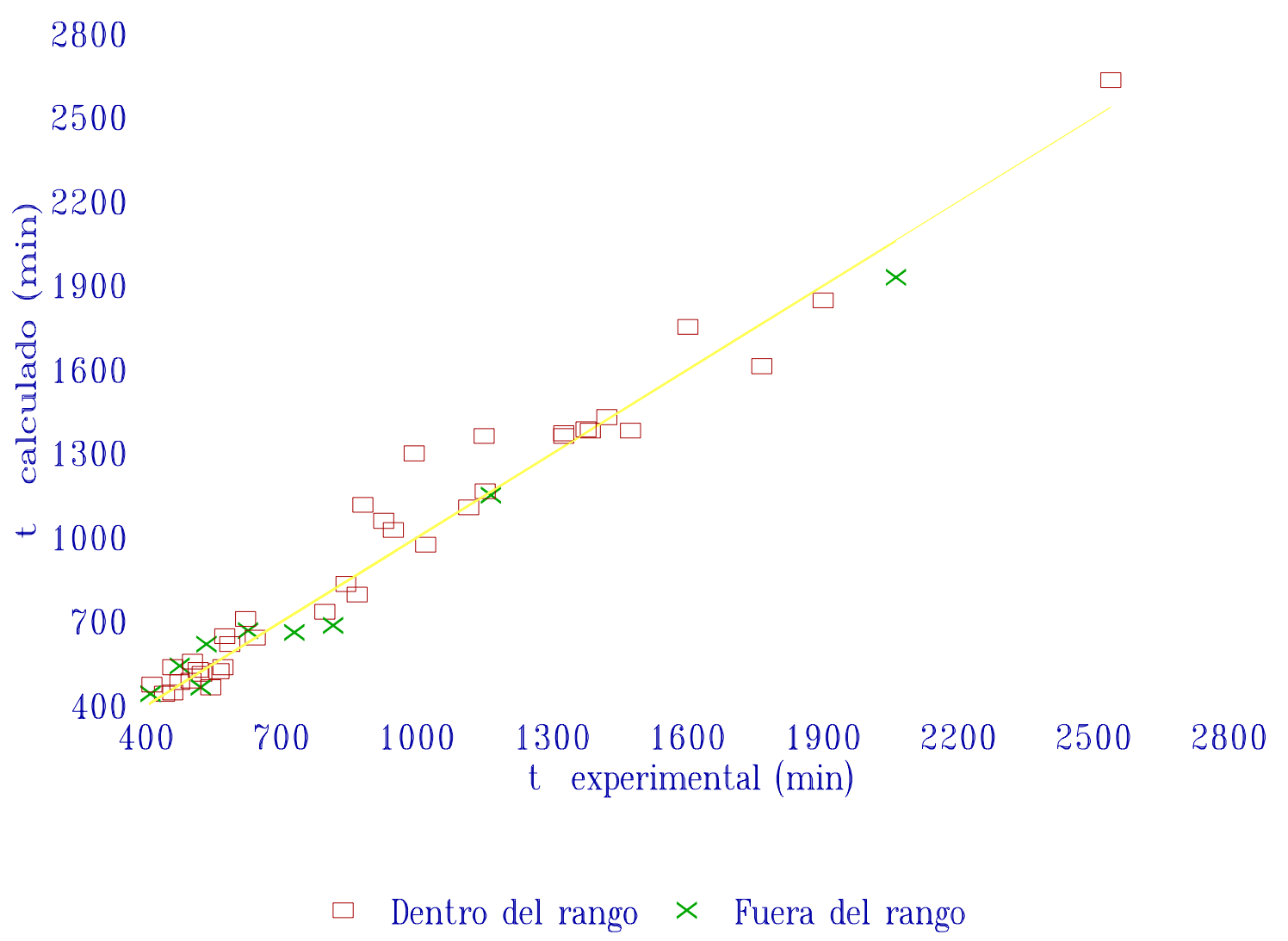

Figura 3.6b. Tiempos de descongelación calculados vs. experimentales, placa plana, diferenciados según sus condiciones operativas estén dentro o fuera del rango de estricta validez del método de predicción.

Cap. $3-22$ 
Tiempos de descongelación

Tabla 3.18. Tiempos de congel ación, experimentales y calculados. Cilindro infinito, tilosa (Cleland y col., 1986).

\begin{tabular}{|c|c|c|c|c|c|c|c|c|}
\hline $\begin{array}{c}\mathrm{L} \\
(\mathrm{cm})\end{array}$ & $\begin{array}{c}h \\
W /\left(m^{2 \circ} \mathrm{C}\right.\end{array}$ & $\mathrm{Bi}$ & $\begin{array}{c}\mathrm{T}_{\mathrm{a}} \\
\left({ }^{\circ} \mathrm{C}\right)\end{array}$ & $\begin{array}{c}\mathrm{T}_{\mathrm{i}} \\
\left({ }^{\circ} \mathrm{C}\right)\end{array}$ & $\begin{array}{c}\mathrm{T}_{\mathrm{c}} \\
\left({ }^{\circ} \mathrm{C}\right)\end{array}$ & $\begin{array}{c}t_{d,}, \exp \\
(\min )\end{array}$ & $\begin{array}{l}t_{d}, \text { pre } \\
\text { (min) }\end{array}$ & $\begin{array}{c}\mathrm{e} \\
(\%)\end{array}$ \\
\hline 7.90 & 23.5 & 3.38 & 43.3 & -14.0 & 0.0 & 440.4 & 469.6 & 6.63 \\
\hline 7.90 & 23.5 & 3.38 & 21.1 & -20.6 & 0.0 & 745.8 & 777.9 & 4.31 \\
\hline 7.90 & 23.5 & 3.38 & 5.1 & -28.4 & 0.0 & 2064.6 & 1953.7 & -5.37 \\
\hline 7.80 & 90.7 & 12.86 & 43.2 & -11.9 & 0.0 & 318.6 & 318.6 & -0.02 \\
\hline 7.80 & 90.7 & 12.86 & 13.0 & -13.6 & 0.0 & 738.0 & 721.4 & -2.25 \\
\hline 7.80 & 90.7 & 12.86 & 5.1 & -27.9 & 0.0 & 1424.4 & 1329.9 & -6.63 \\
\hline 7.80 & 43.5 & 6.17 & 40.3 & -21.2 & 0.0 & 365.4 & 397.7 & 8.84 \\
\hline 7.80 & 43.5 & 6.17 & 11.9 & -27.4 & 0.0 & 924.0 & 913.2 & -1.17 \\
\hline 7.80 & 43.5 & 6.17 & 8.2 & -26.9 & 0.0 & 1152.6 & 1159.0 & 0.56 \\
\hline 7.80 & 43.5 & 6.17 & 5.3 & -14.9 & 0.0 & 1542.0 & 1484.9 & -3.70 \\
\hline 7.63 & 28.0 & 3.88 & 21.1 & -15.2 & 0.0 & 597.0 & 679.4 & 13.80 \\
\hline 7.63 & 28.0 & 3.88 & 20.8 & -16.6 & 0.0 & 636.0 & 688.0 & 8.18 \\
\hline 7.63 & 28.0 & 3.88 & 13.7 & -13.7 & 0.0 & 827.4 & 903.1 & 9.15 \\
\hline 7.63 & 28.0 & 3.88 & 18.6 & -17.2 & 0.0 & 967.2 & 742.7 & -23.21 \\
\hline 7.63 & 28.0 & 3.88 & 5.6 & -16.1 & 0.0 & 1573.8 & 1599.7 & 1.64 \\
\hline 5.30 & 113.0 & 10.89 & 43.3 & -10.7 & 0.0 & 148.2 & 150.4 & 1.46 \\
\hline 5.30 & 113.0 & 10.89 & 8.5 & -20.2 & 0.0 & 476.4 & 456.4 & -4.19 \\
\hline 5.30 & 113.0 & 10.89 & 5.1 & -28.8 & 0.0 & 681.6 & 631.8 & $-7.31 \|$ \\
\hline 5.25 & 34.2 & 3.26 & 21.1 & -15.7 & 0.0 & 307.8 & 345.3 & 12.19 \\
\hline 5.25 & 34.2 & 3.26 & 13.6 & -12.7 & 0.0 & 431.4 & 459.6 & 6.53 \\
\hline 5.25 & 34.2 & 3.26 & 8.5 & -12.6 & 0.0 & 561.0 & 622.6 & 10.97 \\
\hline 5.25 & 34.2 & 3.26 & 5.6 & -32.5 & 0.0 & 910.2 & 831.6 & -8.64 \\
\hline 5.25 & 20.3 & 1.94 & 5.3 & -28.1 & 0.0 & 1129.8 & 1099.5 & -2.68 \\
\hline 5.15 & 37.4 & 3.50 & 43.3 & -30.5 & 0.0 & 195.6 & 202.0 & 3.27 \\
\hline 5.15 & 37.4 & 3.50 & 13.0 & -14.5 & 0.0 & 435.0 & 444.8 & 2.25 \\
\hline 5.15 & 37.4 & 3.50 & 5.3 & -10.6 & 0.0 & 833.4 & 773.7 & -7.16 \\
\hline 5.15 & 25.1 & 2.35 & 40.3 & -10.6 & 0.0 & 227.4 & 244.9 & 7.69 \\
\hline 5.15 & 25.1 & 2.35 & 18.7 & -14.1 & 0.0 & 397.8 & 417.4 & 4.93 \\
\hline 5.15 & 25.1 & 2.35 & 13.2 & -14.4 & 0.0 & 530.4 & 526.5 & -0.74 \\
\hline 5.15 & 25.1 & 2.35 & 5.3 & -31.2 & 0.0 & 1003.8 & 959.9 & -4.37 \\
\hline 5.15 & 19.5 & 1.83 & 43.9 & -26.9 & 0.0 & 259.8 & 272.0 & 4.71 \\
\hline 5.15 & 19.5 & 1.83 & 18.3 & -14.9 & 0.0 & 452.4 & 484.4 & 7.08 \\
\hline 5.15 & 19.5 & 1.83 & 5.8 & -13.1 & 0.0 & 1092.0 & 1008.3 & -7.66 \\
\hline 2.55 & 46.5 & 2.16 & 44.0 & -10.6 & 0.0 & 52.2 & 59.0 & 12.99 \\
\hline 2.55 & 46.5 & 2.16 & 8.5 & -11.9 & 0.0 & 175.8 & 177.8 & 1.16 \\
\hline 2.55 & 46.5 & 2.16 & 5.1 & -10.0 & 0.0 & 253.2 & 241.8 & -4.51 \\
\hline 2.55 & 27.9 & 1.29 & 40.3 & -11.8 & 0.0 & 78.0 & 85.0 & 8.91 \\
\hline 2.55 & 27.9 & 1.29 & 18.9 & -26.5 & 0.0 & 149.4 & 146.3 & -2.06 \\
\hline 2.55 & 27.9 & 1.29 & 13.2 & -18.5 & 0.0 & 180.0 & 183.5 & 1.96 \\
\hline 2.55 & 27.9 & 1.29 & 5.3 & -28.0 & 0.0 & 357.6 & 330.6 & -7.56 \\
\hline 2.55 & 19.0 & 0.88 & 43.9 & -28.1 & 0.0 & 98.4 & 107.2 & 8.97 \\
\hline 2.55 & 19.0 & 0.88 & 14.6 & -18.2 & 0.0 & 225.6 & 223.1 & -1.09 \\
\hline 2.55 & 19.0 & 0.88 & 9.6 & -28.2 & 0.0 & 301.8 & 297.6 & -1.40 \\
\hline 2.55 & 19.0 & 0.88 & 5.8 & -12.1 & 0.0 & 429.0 & 395.8 & -7.75 \\
\hline
\end{tabular}

Cap. $3-23$ 
Tabla 3.19. Tiempos de descongelación, experimentales y calculados. Cilindro infinito, carne picada, Yo=0.68 (llicali, 1989).

\begin{tabular}{|c|c|c|c|c|c|c|c|c|}
\hline $\begin{array}{c}\mathrm{L} \\
(\mathrm{cm})\end{array}$ & $\begin{array}{c}h \\
\mathrm{~W} /\left(\mathrm{m}^{2 \circ} \mathrm{C}\right. \\
) \\
\end{array}$ & $\mathrm{Bi}$ & $\begin{array}{c}\mathrm{T}_{\mathrm{a}} \\
\left({ }^{\circ} \mathrm{C}\right)\end{array}$ & $\begin{array}{c}\mathrm{T}_{\mathrm{i}} \\
\left({ }^{\circ} \mathrm{C}\right)\end{array}$ & $\begin{array}{c}\mathrm{T}_{\mathrm{c}} \\
\left({ }^{\circ} \mathrm{C}\right)\end{array}$ & $\begin{array}{l}t_{d}, \exp \\
\text { (min) }\end{array}$ & $\begin{array}{l}t_{d}, \text { pre } \\
\text { (min) }\end{array}$ & $\begin{array}{c}\mathrm{e} \\
(\%)\end{array}$ \\
\hline 2.20 & 20.8 & 1.05 & 31.6 & -24.6 & 5.0 & 99.0 & 104.7 & 5.79 \\
\hline 2.25 & 20.8 & 1.08 & 26.6 & -20.2 & 5.0 & 125.5 & 120.6 & -3.94 \\
\hline 2.25 & 54.4 & 2.82 & 27.2 & -24.8 & 5.0 & 63.5 & 69.0 & 8.71 \\
\hline 2.25 & 54.4 & 2.82 & 27.4 & -28.8 & 5.0 & 66.0 & 69.0 & 4.59 \\
\hline 2.15 & 66.0 & 3.27 & 27.4 & -28.4 & 5.0 & 58.7 & 59.1 & 0.64 \\
\hline
\end{tabular}

Tabla 3.20. Tiempos de descongelación, experimentales y calculados. Cilindro infinito, puré de papa (llicali, 1989).

\begin{tabular}{|c|c|c|c|c|c|c|c|c|}
\hline $\begin{array}{c}\mathrm{L} \\
(\mathrm{cm})\end{array}$ & $\begin{array}{c}h \\
W /\left(m^{2 \circ} \mathrm{C}\right.\end{array}$ & $\mathrm{Bi}$ & $\begin{array}{c}\mathrm{Ta}_{\mathrm{a}} \\
\left({ }^{\circ} \mathrm{C}\right)\end{array}$ & $\begin{array}{c}\mathrm{T}_{\mathrm{i}} \\
\left({ }^{\circ} \mathrm{C}\right)\end{array}$ & $\begin{array}{c}\mathrm{T}_{\mathrm{c}} \\
\left({ }^{\circ} \mathrm{C}\right)\end{array}$ & $\begin{array}{l}t_{d}, \exp \\
(\min )\end{array}$ & $\begin{array}{l}\mathrm{t}_{\mathrm{d}, \mathrm{pre}} \\
\text { (min) }\end{array}$ & $\begin{array}{c}\mathrm{e} \\
(\%)\end{array}$ \\
\hline 2.15 & 20.8 & 0.84 & 28.4 & -20.2 & 5.0 & 120.0 & 113.2 & -5.67 \\
\hline 2.25 & 20.8 & 0.88 & 29.8 & -24.2 & 5.0 & 132.5 & 116.7 & -11.89 \\
\hline 2.20 & 54.4 & 2.26 & 26.4 & -24.8 & 5.0 & 65.0 & 67.7 & 4.17 \\
\hline 2.20 & 54.4 & 2.26 & 26.4 & -23.2 & 5.0 & 68.0 & 67.6 & -0.65 \\
\hline 2.20 & 66.0 & 2.74 & 27.4 & -33.0 & 5.0 & 67.0 & 60.7 & -9.42 \\
\hline 2.10 & 66.0 & 2.62 & 27.4 & -26.4 & 5.0 & 59.5 & 56.1 & -5.73 \\
\hline
\end{tabular}


2000

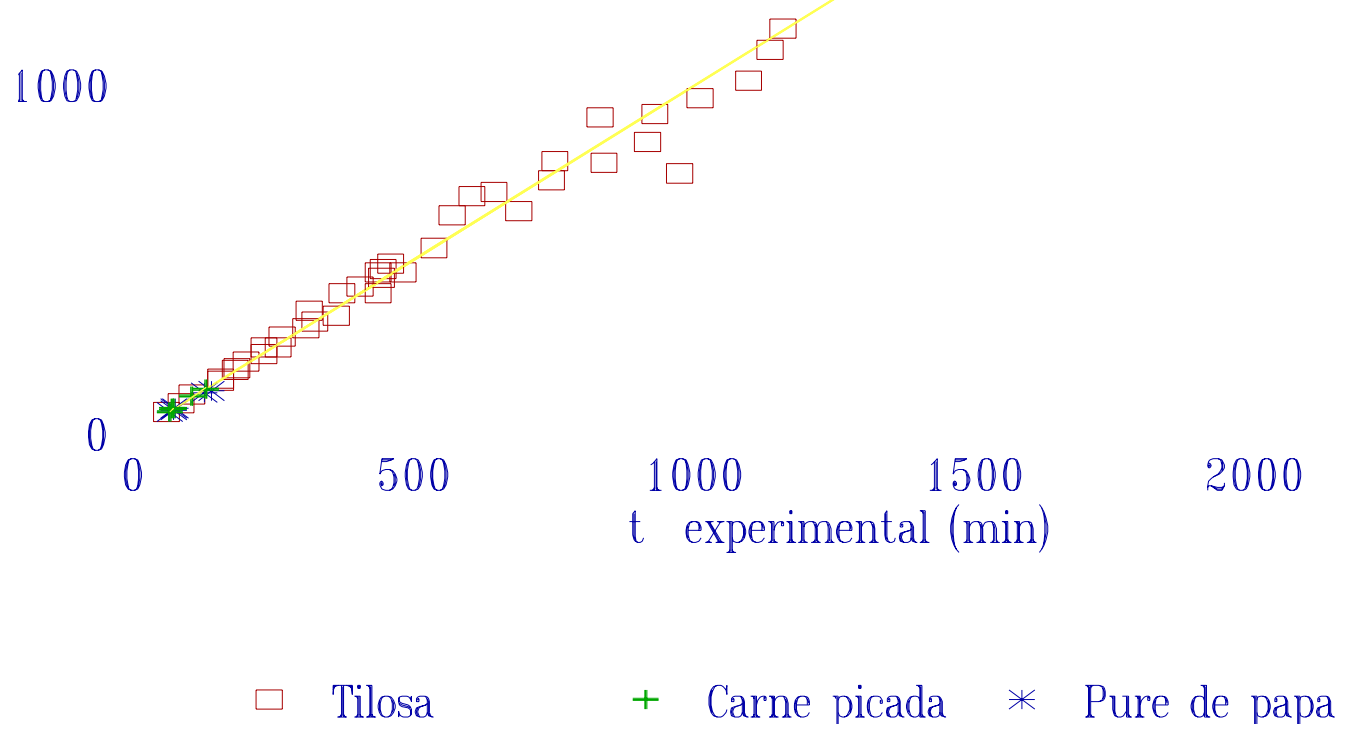

Figura 3.7. Tiempos de descongelación calculados vs. experimentales, cilindro infinito, diferenciados por tipo de producto.

Cap. $3-25$ 


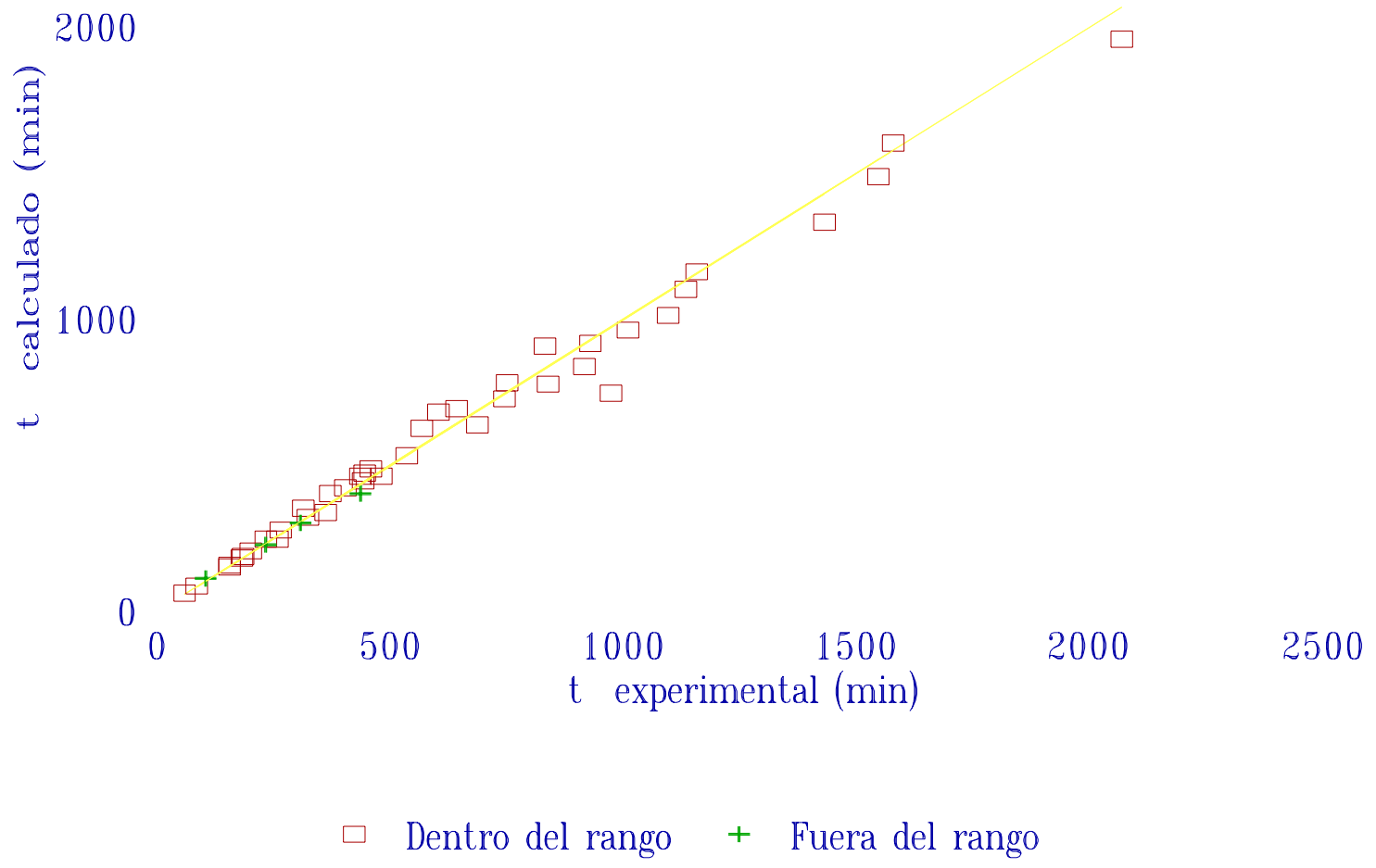

Figura 3.8. Tiempos de descongelación calculados vs. experimentales, cilindro infinito, diferenciados según sus condiciones operativas estén dentro o fuera del rango de estricta validez del método de predicción. 
Tiempos de descongelación

Tabla 3.21. Tiempos de descongelación, experimentales y calculados. Esfera, tilosa (Cleland y col., 1986).

\begin{tabular}{|c|c|c|c|c|c|c|c|c|}
\hline $\begin{array}{c}\mathrm{L} \\
(\mathrm{cm})\end{array}$ & $\begin{array}{c}h \\
\mathrm{~W} /\left(\mathrm{m}^{2 \circ} \mathrm{C}\right. \\
)\end{array}$ & $\mathrm{Bi}$ & $\begin{array}{c}\mathrm{T}_{\mathrm{a}} \\
\left({ }^{\circ} \mathrm{C}\right)\end{array}$ & $\begin{array}{c}\mathrm{T}_{\mathrm{i}} \\
\left({ }^{\circ} \mathrm{C}\right)\end{array}$ & $\begin{array}{c}\mathrm{T}_{\mathrm{c}} \\
\left({ }^{\circ} \mathrm{C}\right)\end{array}$ & $\begin{array}{l}t_{d}, \exp \\
(\min )\end{array}$ & $\begin{array}{l}t_{d}, \text { pre } \\
\text { (min) }\end{array}$ & $\begin{array}{c}\mathrm{e} \\
(\%)\end{array}$ \\
\hline 6.40 & 246.2 & 28.65 & 43.3 & -9.7 & 0.0 & 135.6 & 136.3 & 0.54 \\
\hline 6.40 & 246.2 & 28.65 & 21.1 & -25.3 & 0.0 & 235.2 & 223.5 & -4.97 \\
\hline 6.40 & 246.2 & 28.65 & 13.0 & -19.7 & 0.0 & 325.2 & 301.5 & -7.29 \\
\hline 6.40 & 246.2 & 28.65 & 44.0 & -15.1 & 0.0 & 159.6 & 136.7 & -14.36 \\
\hline 6.40 & 74.8 & 8.70 & 18.3 & -18.8 & 0.0 & 286.8 & 286.6 & -0.08 \\
\hline 6.40 & 74.8 & 8.70 & 11.9 & -23.1 & 0.0 & 383.4 & 377.7 & -1.48 \\
\hline 6.40 & 74.8 & 8.70 & 5.3 & -26.5 & 0.0 & 679.8 & 613.3 & -9.78 \\
\hline 6.40 & 51.6 & 6.00 & 43.3 & -27.9 & 0.0 & 165.6 & 182.3 & 10.10 \\
\hline 6.40 & 51.6 & 6.00 & 22.0 & -18.8 & 0.0 & 258.6 & 279.9 & 8.25 \\
\hline 6.40 & 51.6 & 6.00 & 14.5 & -20.3 & 0.0 & 348.6 & 365.6 & 4.87 \\
\hline 6.40 & 51.6 & 6.00 & 5.1 & -15.5 & 0.0 & 735.6 & 678.3 & -7.79 \\
\hline 6.40 & 51.6 & 6.00 & 43.6 & -20.3 & 0.0 & 187.8 & 179.9 & -4.21 \\
\hline 6.40 & 41.9 & 4.88 & 12.0 & -33.0 & 0.0 & 457.8 & 445.0 & -2.79 \\
\hline 6.40 & 41.9 & 4.88 & 5.5 & -17.1 & 0.0 & 769.2 & 695.5 & -9.58 \\
\hline 6.35 & 51.4 & 5.93 & 12.8 & -11.9 & 0.0 & 356.4 & 384.4 & 7.87| \\
\hline 6.35 & 51.4 & 5.93 & 5.6 & -20.9 & 0.0 & 668.4 & 640.9 & -4.11 \\
\hline 5.60 & 76.0 & 7.74 & 43.9 & -14.5 & 0.0 & 113.4 & 127.0 & 11.97 \\
\hline 5.60 & 76.0 & 7.74 & 8.0 & -13.9 & 0.0 & 357.6 & 372.9 & 4.27| \\
\hline 5.60 & 76.0 & 7.74 & 5.3 & -28.0 & 0.0 & 472.2 & 483.2 & 2.33 \\
\hline 5.60 & 59.4 & 6.05 & 43.6 & -27.1 & 0.0 & 121.2 & 138.6 & 14.33 \\
\hline 5.60 & 59.4 & 6.05 & 14.5 & -16.6 & 0.0 & 243.0 & 277.7 & 14.29 \\
\hline 5.60 & 59.4 & 6.05 & 5.0 & -18.2 & 0.0 & 541.2 & 526.4 & -2.73 \\
\hline 5.60 & 45.7 & 4.65 & 43.6 & -30.2 & 0.0 & 145.8 & 151.0 & 3.57 \\
\hline 5.60 & 45.7 & 4.65 & 8.9 & -32.9 & 0.0 & 430.8 & 415.6 & -3.53 \\
\hline 5.60 & 45.7 & 4.65 & 5.5 & -17.4 & 0.0 & 580.2 & 541.3 & -6.70 \\
\hline 2.80 & 137.2 & 6.98 & 18.3 & -16.9 & 0.0 & 51.6 & 57.6 & 11.71 \\
\hline 2.80 & 137.2 & 6.98 & 11.9 & -13.4 & 0.0 & 69.0 & 75.0 & 8.74 \\
\hline 2.80 & 137.2 & 6.98 & 5.3 & -23.5 & 0.0 & 124.8 & 123.3 & -1.18 \\
\hline 2.80 & 87.0 & 4.43 & 43.6 & -14.2 & 0.0 & 34.8 & 37.6 & 8.03 \\
\hline 2.80 & 87.0 & 4.43 & 22.2 & -20.3 & 0.0 & 52.2 & 58.9 & 12.81 \\
\hline 2.80 & 87.0 & 4.43 & 14.5 & -14.8 & 0.0 & 66.6 & 76.5 & 14.86 \\
\hline 2.80 & 87.0 & 4.43 & 7.0 & -22.8 & 0.0 & 112.8 & 120.7 & 7.01 \\
\hline 2.80 & 87.0 & 4.43 & 5.0 & -24.7 & 0.0 & 139.8 & 146.7 & 4.96 \\
\hline 2.80 & 57.5 & 2.93 & 43.6 & -22.8 & 0.0 & 40.2 & 45.1 & 12.12 \\
\hline 2.80 & 57.5 & 2.93 & 22.3 & -14.9 & 0.0 & 63.6 & 68.8 & 8.24 \\
\hline 2.80 & 57.5 & 2.93 & 12.1 & -21.7 & 0.0 & 94.8 & 102.4 & 8.02 \\
\hline 2.80 & 57.5 & 2.93 & 5.5 & -16.3 & 0.0 & 160.2 & 162.6 & 1.47 \\
\hline
\end{tabular}

Cap. $3-27$ 
Tabla 3.22. Tiempos de descongelación, experimentales y calculados. Esfera, carne picada, Yo $=0.68$ (Ilicali, 1989).

\begin{tabular}{|c|c|c|c|c|c|c|c|c|}
\hline $\begin{array}{c}\mathrm{L} \\
(\mathrm{cm})\end{array}$ & $\begin{array}{c}h \\
\mathrm{~W} /\left(\mathrm{m}^{2 \circ} \mathrm{C}\right. \\
)\end{array}$ & $\mathrm{Bi}$ & $\begin{array}{c}\mathrm{Ta}_{\mathrm{a}} \\
\left({ }^{\circ} \mathrm{C}\right)\end{array}$ & $\begin{array}{c}\mathrm{T}_{\mathrm{i}} \\
\left({ }^{\circ} \mathrm{C}\right)\end{array}$ & $\begin{array}{c}\mathrm{T}_{\mathrm{c}} \\
\left({ }^{\circ} \mathrm{C}\right)\end{array}$ & $\begin{array}{l}t_{d}, \exp \\
(\min )\end{array}$ & $\begin{array}{l}t_{d}, \text { pre } \\
\text { (min) }\end{array}$ & $\begin{array}{c}\mathrm{e} \\
(\%)\end{array}$ \\
\hline 2.95 & 15.0 & 1.02 & 31.2 & -24.2 & 5.0 & 127.8 & 135.5 & 6.00 \\
\hline 2.90 & 15.0 & 1.00 & 27.6 & -25.6 & 5.0 & 128.0 & 143.7 & 12.27 \\
\hline 2.90 & 38.0 & 2.54 & 32.4 & -22.4 & 5.0 & 69.0 & 73.6 & 6.65 \\
\hline 2.90 & 38.0 & 2.54 & 28.8 & -21.2 & 5.0 & 70.5 & 79.3 & 12.51 \\
\hline 2.95 & 50.0 & 3.40 & 27.6 & -35.6 & 5.0 & 65.5 & 75.0 & 14.46 \\
\hline 2.90 & 50.0 & 3.34 & 28.7 & -32.6 & 5.0 & 64.6 & 71.0 & 9.87 \\
\hline
\end{tabular}

Tabla 3.23. Tiempos de descongelación, experimentales y calculados. Esfera, puré de papa (llicali, 1989)

\begin{tabular}{|c|c|c|c|c|c|c|c|c|}
\hline $\begin{array}{c}\mathrm{L} \\
(\mathrm{cm})\end{array}$ & $\begin{array}{c}\mathrm{h} \\
\mathrm{W} /\left(\mathrm{m}^{2 \circ} \mathrm{C}\right. \\
)\end{array}$ & $\mathrm{Bi}$ & $\begin{array}{c}\mathrm{T}_{\mathrm{a}} \\
\left({ }^{\circ} \mathrm{C}\right)\end{array}$ & $\begin{array}{c}\mathrm{T}_{\mathrm{i}} \\
\left({ }^{\circ} \mathrm{C}\right)\end{array}$ & $\begin{array}{c}\mathrm{T}_{\mathrm{c}} \\
\left({ }^{\circ} \mathrm{C}\right)\end{array}$ & $\begin{array}{l}t_{d}, \exp \\
(\min )\end{array}$ & $\begin{array}{l}t_{d,}, \text { pre } \\
\text { (min) }\end{array}$ & $\begin{array}{c}\mathrm{e} \\
(\%)\end{array}$ \\
\hline 2.95 & 17.5 & 0.97 & 30.8 & -25.6 & 5.0 & 113.5 & 127.7 & 12.50 \\
\hline 2.95 & 17.5 & 0.97 & 31.0 & -24.4 & 5.0 & 117.0 & 127.0 & 8.53 \\
\hline 2.60 & 16.0 & 0.78 & 30.4 & -24.8 & 5.0 & 120.5 & 117.2 & -2.76 \\
\hline 2.90 & 45.0 & 2.46 & 27.4 & -32.8 & 5.0 & 75.8 & 76.1 & 0.36 \\
\hline 2.95 & 45.0 & 2.50 & 26.8 & -24.8 & 5.0 & 81.7 & 78.6 & -3.84 \\
\hline 2.95 & 32.0 & 1.78 & 28.6 & -23.8 & 5.0 & 85.5 & 90.4 & 5.67 \\
\hline
\end{tabular}

Cap. $3-28$ 
300

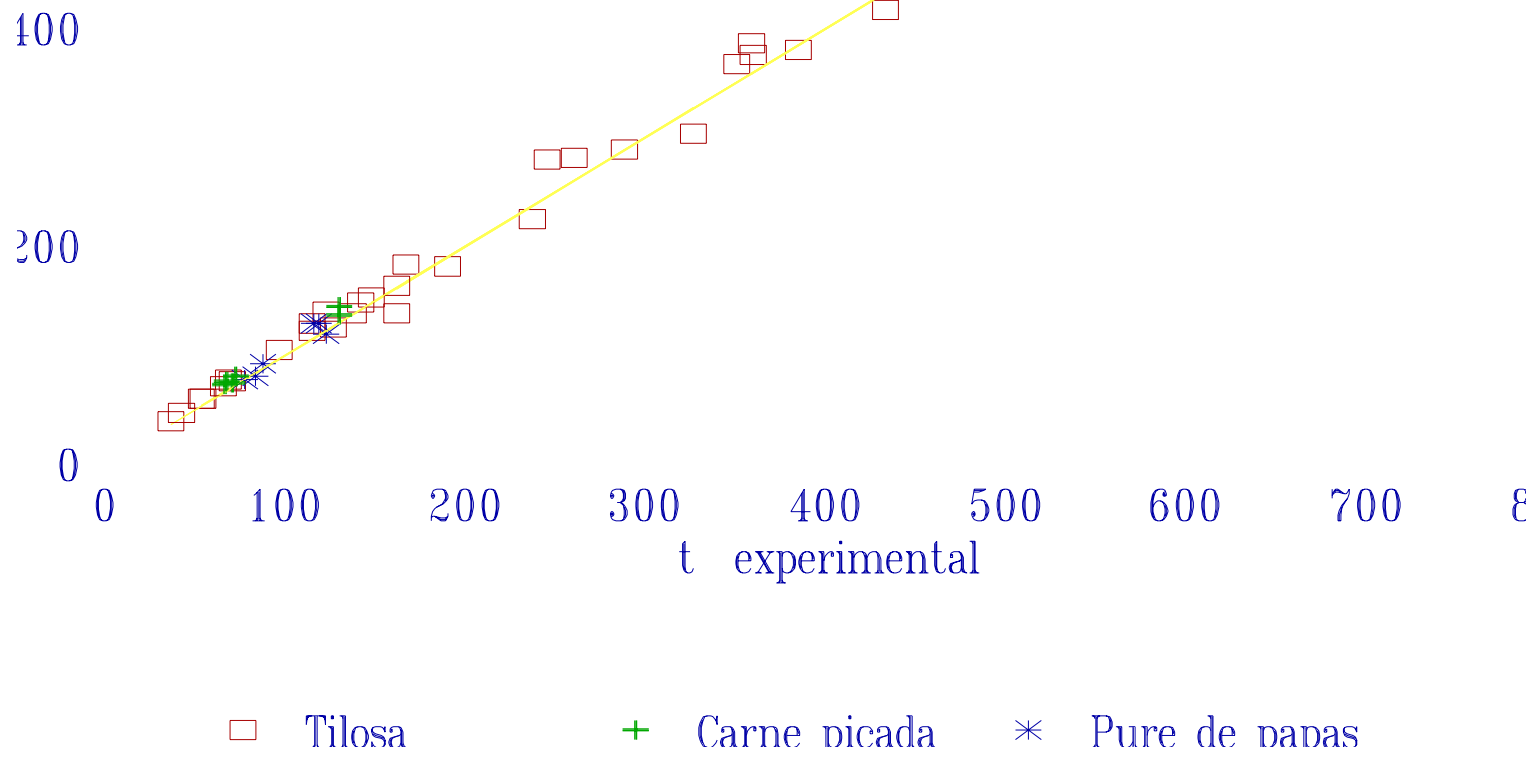

Figura 3.9. Tiempos de descongelación calculados vs. experimentales, esfera, diferenciados por tipo de producto.

Cap. 3 -29 


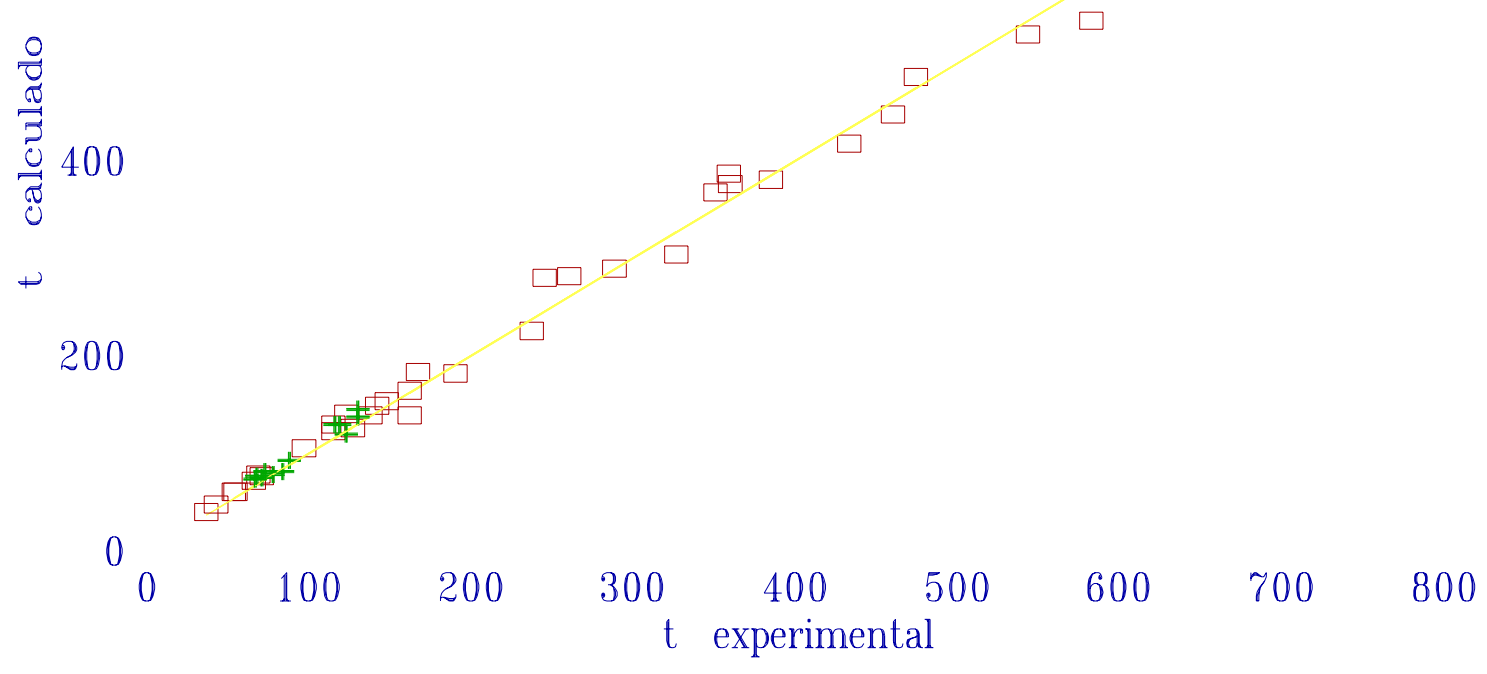

Figura 3.10. Tiempos de congelación cal culados vs. experimentales, esfera; diferenciados según sus condiciones operativas estén dentro o fuera del rango de estricta validez del método de predicción.

Análogamente a lo realizado durante la predicción de tiempos de congelación, para evaluar la bondad del método se han comparado los tiempos de descongelación experimentales con los tiempos calculados con la metodología propuesta.

La última columna de las Tablas 3.6 a 3.23 presenta el error porcentual e definido en la ec. (2.63). En la Tabla siguiente se detalla el error porcentual promedio _, la desviación estándar y los límites de confianza (correspondientes al 95\%), para cada geometría estudiada.

Tabla 3.24. Error promedio y desviación estándar. 


\begin{tabular}{||lrcccc||}
\hline Geometría & $\begin{array}{c}\text { Númer } \\
\text { o } \\
\text { de } \\
\text { datos }\end{array}$ & $\begin{array}{c}\text { Desv. } \\
(\%)\end{array}$ & $\begin{array}{c}\text { Estándar } \\
(\%)\end{array}$ & $\begin{array}{c}\text { Límite } \\
\text { Superior } \\
(\%)\end{array}$ & $\begin{array}{c}\text { Límite } \\
\text { Inferior } \\
(\%)\end{array}$ \\
\hline \hline Placa plana & 104 & 1.87 & 9.072 & 20.91 & -16.13 \\
Cil. infinito & 55 & 0.46 & 7.215 & 15.60 & -13.84 \\
Esfera & 49 & 3.71 & 7.647 & 18.46 & -13.06 \\
Todos los datos & 208 & 1.93 & 8.332 & 18.59 & -14.73 \\
\hline \hline
\end{tabular}

\subsection{CONCLUSIONES}

Se ha desarrollado un método de predicción de tiempos de descongelación de alimentos, válido para las formas regulares. La principal ventaja del método es que con una única ecuación,se obtienen resultados de igual precisión a los obtenidos con modelos aproximados más complicados o inclusive con modelos numéricos.

Se ha probado que la ec. (3.10) es válida para un rango amplísimo de condiciones operativas, cubriendo extensamente las condiciones más frecuentes en la descongelación industrial. La sencillez del método permite evaluar rápidamente como varía el tiempo de descongelación con las condiciones operativas que debe fijar el responsable del procesamiento de los productos.

Se ha probado también que el método es válido para distintos alimentos, de composición, estructura y propiedades muy diferentes, como carne vacuna entera, carne picada, cordero, pescados, geles. En general puede extenderse a todos aquellos alimentos que presenten un al to contenido inicial de agua.

La precisión del método es muy buena, teniendo en cuenta que además de haberse verificado con distintos alimentos, en algunos casos las condiciones experimentales no se mantuvieron constantes, los valores de las propiedades físicas y el coeficiente de transferencia calórica son dudosos, etc. 
La simplicidad del método lo hace especialmente adecuado para realizar cálculos industriales, cuando no se cuenta con toda la información sobre la variación de las propiedades termofísicas con la temperatura. Para utilizar la ec. (3.10) sólo se deben conocer la difusividad térmica y la conductividad térmica del alimento fresco, que son los valores más conocidos. 
CAPITULO IV

ALM ACENAM IENTO 


\subsection{INTRODUCCION}

En general los alimentos congelados sufren un período de almacenamiento prolongado antes de ser consumidos. En particular en el presente capítulo se analiza la influencia de las distintas variables que provocan la pérdida de calidad que se produce durante el al macenamiento, atributo que está íntimamente ligado a la vida útil del producto y, por consiguiente a su valor comercial.

La variable que afecta principal mente a la calidad del alimento, además de la duración del almacenamiento, es la temperatura del ambiente de almacenamiento, y en especial el hecho de que se produzcan fluctuaciones de la misma durante dicho período (Singh \& Wang, 1977).

En virtud de lo señalado previamente, se ha desarrollado una metodología de cálculo que permite predecir la temperatura de distintas estibas de alimentos congelados cuando varía la temperatura del ambiente de almacenamiento. Se estudian también los distintos factores que suelen utilizarse como indicadores de la calidad de los productos congelados, y se relaciona esta última con la temperatura y el tiempo dealmacenamiento.

\subsection{FUNCIONES DE TRANSFEREN CIA}

Para estudiar la influencia de las variaciones térmicas, que se producen tanto durante el almacenamiento de los productos congelados como en las etapas posteriores de distribución y exposición en los lugares de venta, es necesario poder predecir la respuesta que tendrán los productos almacenados a diferentes fluctuaciones. El cálculo de las historias térmicas resulta difícil ya que se presentan estibas con estructura compleja (material de estiba + empaque + alimento), el producto es heterogéneo, existe anisotropía, las condiciones de contorno varían con el tiempo, etc.

Debido a las características citadas del sistema a estudiar en la mayoría de las situaciones no se conoce ni existe una solución analítica. En los pocos casos en que se puede calcular, para condiciones de contorno variables en el tiempo, la temperatura de una posición determinada en función del tiempo, la solución 
analítica consiste en una sumatoria infinita cuyo cálculo para cada nueva situación es muy engorroso. Por ejemplo, Zaritzky (1982) compara los resultados obtenidos con una solución analítica aproximada y una solución numérica al modelar el almacenamiento y distribución de productos cárneos de formas uni y tridimensionales.

En cuanto a los modelos numéricos, no resulta sencillo plantearlos ya que se debe tener en cuenta que los sistemas suelen ser heterogéneos, generalmente presentan formas irregulares (como la estiba de baldes que analizaremos posteriormente), y es imprescindible conocer con precisión las funciones que vinculan las propiedades físicas con la temperatura y el coeficiente de transferencia calórica para obtener buenos resultados. Por ejemplo, Zuritz \& Singh (1985) utilizan un esquema de elementos finitos para estudiar el comportamiento térmico de alimentos envasados en contenedores cilíndricos. Simulan las condiciones de transporte provocando dos escalones arbitrarios de temperatura, a $28^{\circ} \mathrm{C}$ y $9^{\circ} \mathrm{C}$. Experimental mente miden la respuesta de helado comercial y tilosa.

En general, utilizando métodos numéricos, para calcular las respuestas térmicas a diferentes perturbaciones será necesario ejecutar el programa de cálculo una vez para cada nueva situación lo cual, si bien en los últimos tiempos ha mejorado notablemente debido a la gran velocidad de cálculo de las computadoras actuales, puede significar horas de tiempo de máquina. Esto se debe a que se simulan sistemas con transmisión de calor multidimensional (que implica trabajar con grandes matrices) y experiencias que general mente duran varios días.

Uno de los objetivos del presente trabajo es demostrar la potencialidad de las funciones de transferencia z para caracterizar sistemas sujetos a transferencia de calor, con condiciones de contorno variables en el tiempo. La metodología de cálculo consiste en modelar el sistema de interés, obteniendo su función de transferencia, lo que permitirá mediante cálculos muy sencillos predecir respuestas del mismo a diferentes perturbaciones externas.

La caracterización de sistemas mediante sus respectivas funciones de transferencia ha sido extensamente empleada por la teoría de control automático de procesos. En lo que respecta a problemas de transferencia de calor, la primera 


\section{Almacenamiento}

aplicación sistematizada aparece en la simulación de paredes multicapa, en el diseño de sistemas de refrigeración y calefacción de edificios (Stephenson \& Mitalas, 1971). Hoy en día el uso de esta metodología en este campo se encuentra ampliamente difundido (ASHRAE, 1985). Recientemente, varios autores han aplicado las funciones de transferencia para resolver problemas específicos de transferencia de calor, uni- o multidimensionales (Mitalas (1978), Sanz (1984), Sanz y col. (1986), Barakat (1987), Ceylan (1987), Mascheroni y col. (1987), Seem y col. (1989a, 1989b)). Los distintos autores utilizan métodos diferentes para obtener las correspondientes funciones de transferencia, debido a que sus sistemas y objetivos no son los mismos.

\subsubsection{FORMULACION MATEMATICA}

Para un sistema lineal, con propiedades que no varían en el tiempo, la relación existente entre una perturbación $p(t)$ que afecta al sistema y la respuesta del mismo $r(t)$ queda descripta por una ecuación diferencial lineal (ec. (4.1)):

$$
a_{0} \frac{d^{n} r(t)}{d t^{n}}+a_{1} \frac{d^{n-1} r(t)}{d t^{n-1}}+. .+a_{n} r(t)=b_{0} \frac{d^{m} p(t)}{d t^{m}}+b_{1} \frac{d^{m-1} p(t)}{d t^{m-l}}+. .+b_{m} p(t)
$$

Considerando que las funciones $p(t)$ y $r(t)$ son continuas y que los coeficientes ai y bi son constantes, la ec. (4.1) puede transformarse término a término mediante transformadas de Laplace (ec. (4.2)):

$$
\left(a_{0} s^{n}+a_{1} s^{n-1}+\ldots\right) R(s)=\left(b_{0} s^{m}+b_{1} s^{m-1}+\ldots\right) P(s)
$$

El sistema queda entonces caracterizado por su correspondiente función de transferencia, definida en la ec. (4.3), donde se ha supuesto que las funciones $p(t)$ y $r(t)$ y sus derivadas tienen condición inicial nula:

$$
F(s)=\frac{R(s)}{P(s)}
$$

Muestreando la función continua $\mathrm{p}(\mathrm{t})$ a intervalos regulares, siendo $\Delta$ el intervalo de muestreo, se obtiene una nueva función, discontinua, que llamaremos 
$p^{*}(t):$

$$
p^{*}(t)=p(t) \delta_{\Delta}(t)=p(t) \sum_{n=0}^{\infty} \delta(t-n \Delta)
$$

donde la función impulso o delta de Dirac toma el siguiente valor:

$$
\delta_{\Delta}(t)= \begin{cases}1 & \text { si } t=n \Delta \\ 0 & \text { si } t \neq n \Delta\end{cases}
$$

La Figura 4.1 muestra una función continua cualquiera $p(t)$ y su correspondiente función muestreada $\mathrm{p}^{*}(\mathrm{t})$.

Definiendo una nueva variable compleja $z=e^{\mathrm{s}}$, y cal culando la transformada de Laplace de la función muestreada, queda definida la transformada $z$ de la función $p(t)$ :

$$
Z[p(t)]=L\left[p^{*}(t)\right]=\sum_{n=0}^{\infty} p(n \Delta) e^{-n \Delta s}=\sum_{n=0}^{\infty} p(n \Delta) z^{-n}
$$

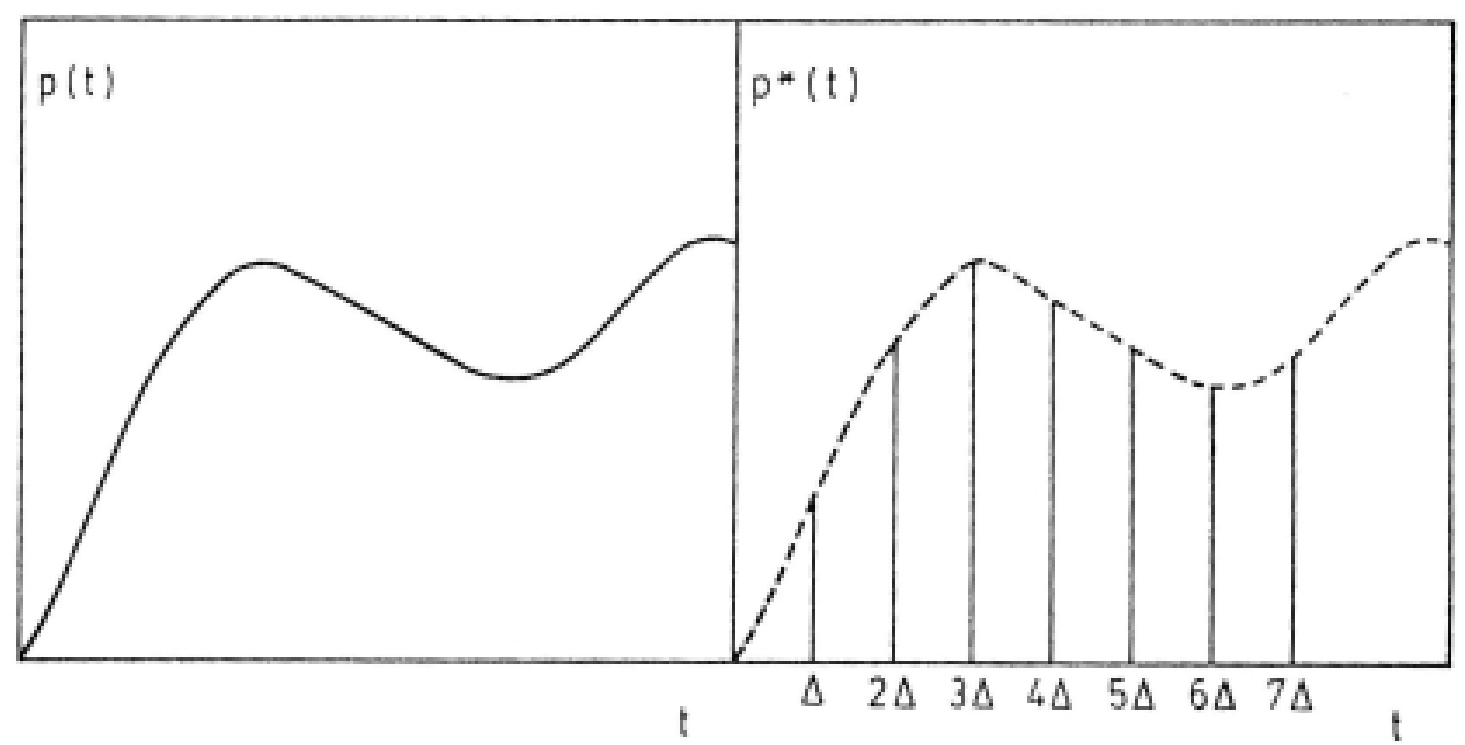

Figura 4.1. Función continua $\mathrm{p}(\mathrm{t})$ y función muestreada $\mathrm{p}^{*}(\mathrm{t})$. 
La principal ventaja que presenta esta transformación, especialmente en el caso de funciones que no tienen una expresión analítica sencilla, es que puede escribirse la sumatoria conociendo únicamente los valores que toma la función $p(t)$ en los instantes de tiempo $t=n \Delta$. Pero no debe olvidarse que mientras el análisis que se efectúa mediante la transformación de Laplace es exacto y válido para todo valor detiempo $t \geq 0$, la transformada $z$ es discontinua (sólo proporciona resultados para t $=\mathrm{n} \Delta$ ) y como generalmente no se trabaja con la serie infinita sino que sólo se consideran al gunos términos de la misma, la solución obtenida será aproximada.

Para un sistema en particular, la definición de función de transferencia z es análoga a la definición de la función de transferencia s, es decir $\mathrm{F}(\mathrm{z})$ es el cociente entre la transformada $z$ de la respuesta $\mathrm{R}(\mathrm{z})$ y la transformada $\mathrm{z}$ de la perturbación, $\mathrm{P}(\mathrm{z})$ :

$$
F(z)=\frac{R(z)}{P(z)}=\frac{r(0)+r(\Delta) z^{-1}+r(2 \Delta) z^{-2}+\ldots .+r(n \Delta) z^{-n}+\ldots}{p(0)+p(\Delta) z^{-1}+p(2 \Delta) z^{-2}+\ldots .+p(n \Delta) z^{-n}+\ldots}
$$

$F(z)$ puede expresarse también como un polinomio en $z^{-n}$ (ec. (4.8)), definiendo los "coeficientes de la función de transferencia" $\mathrm{fn}_{\mathrm{n}}$

$$
F(z)=f_{1}+f_{2} z^{-1}+f_{3} z^{-2}+f_{4} z^{-3}+\ldots=\sum_{n=1}^{\infty} f_{n} z^{n-1}
$$

El modo habitual de determinar los coeficientes $f_{n}$ y por ende $F(z)$, consiste en medir experimentalmente o calcular analítica o numéricamente la respuesta del sistema en estudio a una señal de perturbación patrón.

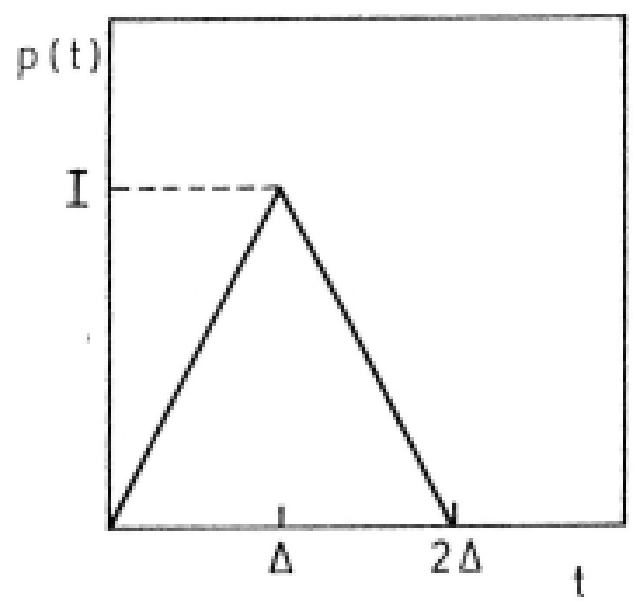

Figura 4.2. Señal pico. 
Una de las señales más utilizadas consiste en un pulso triangular o doble rampa, usualmente llamada pico, de altura I y ancho $2 \Delta$ (Figura 4.2). La gran ventaja de esta señal es que su transformada $z$ sólo tiene un término distinto de cero (ec. 4.9) lo cual simplifica enormemente el cociente de polinomios en $z^{-n}$ de la ec. (4.7):

$$
P(z)=I z^{-1}
$$

y los coeficientes $f_{n}$ resultan:

$$
f_{n}=\frac{r(n \Delta)}{I}
$$

Conocida la función de transferencia del sistema, ésta permitirá predecir la respuesta del sistema frente a cualquier otra perturbación.

De la ec. (4.7) se deduce que la transformada $z$ de la respuesta es igual al producto de la transformada $z$ de la señal de entrada o perturbación y $F(z)$. Para una entrada cualquiera e(t) cuya transformada $z$ es $E(z)$; la transformada $z$ de la respuesta será $S(z)$ :

$$
S(z)=F(z) E(z)=\sum_{n=0}^{\infty} s(n \Delta) z^{-n}
$$

El valor que toma la respuesta $s(t)$ en el instante $t=n \Delta$ es el coeficiente de $z^{-n}$ en $S(z)$ :

$$
s_{n}=s(n \Delta)=f_{1} e(n \Delta)+f_{2} e((n-1) \Delta)+\ldots+f_{n+1} e(0)=\sum_{i=1}^{n+1} f_{i} e((n+1-i) \Delta)
$$

Es importante remarcar que, a pesar de que el cálculo de la respuesta térmica es sencillo, no se puede cal cular la respuesta continua sino sólo los val ores que toma $s(t)$ en $\mathrm{t}=\mathrm{n} \Delta$.

En la práctica $F(z)$, en la ec. (4.8), se aproxima por un número finito de coeficientes, $\mathrm{N}$. El valor de $\mathrm{N}$ afectará la precisión de los resultados predichos. 
Como regla práctica se establece $\mathrm{N}$ de modo tal que:

$$
\sum_{i=1}^{N} f_{i} \geq 0.99
$$

Si N es pequeño, y se quiere calcular la respuesta a tiempos largos, de modo tal que n sea considerablemente mayor que $\mathrm{N}$, el cálculo de la salida s(t) para dichos tiempos desprecia el efecto de los primeros valores de la señal de entrada, introduciendo un nuevo error:

$$
s_{n}=s(n \Delta)=f_{1} e(n \Delta)+f_{2} e((n-1) \Delta)+\ldots+f_{N} e((n+1-N) \Delta)
$$

Si se cumple la ec. (4.13) estaremos despreciando coeficientes de poco peso (en general la serie de la ec. (4.8) es convergente, a medida que se incrementa n los coeficientes tienden a cero) y el error cometido en el cálculo de la respuesta $s(n \triangle)$ será mínimo.

El valor del intervalo de muestreo también afecta la precisión de los cálculos, para determinar $\Delta$ debe contemplarse la inercia térmica del sistema en estudio. De acuerdo al teorema de muestreo (Luyben, 1973) el período mínimo de muestreo para obtener información dinámica de un proceso debe ser menor que la mitad de la menor constante de tiempo del sistema. Cuando se desconoce por completo la dinámica del proceso térmico a simular, como suele ser nuestro caso de estudio, es necesario realizar algunas pruebas experimentales para determinar el intervalo de muestreo $\Delta$. En general podemos decir que el mismo deberá ser considerablemente menor que el período en que se observa una evolución macroscópica del sistema.

En los ejemplos de aplicación que presentamos a continuación siempre hemos considerado como función $r(t) \circ \mathrm{s}(\mathrm{t})$ a la temperatura de un punto representativo del comportamiento térmico del sistema y como función de perturbación $p(t)$ o e $(t)$ a la temperatura del aire en los alrededores del sistema. A mbas temperaturas se han medido respecto de su valor inicial, para cumplir el requisito de que la condición inicial sea nula. 


\subsubsection{EQUIPO EXPERIMENTAL}

\subsubsection{CAMARA EXPERIMENTAL DE ALMACENAMIENTO}

El cuerpo principal del equipamiento (Figura 4.3) consiste en una cámara frigorífica modular marca DISTHEL modelo CT-19, fabricada con paneles de poliuretano inyectado de $100 \mathrm{~mm}$ de espesor. Las dimensiones de la cámara son las siguientes: $2.90 \times 2.52 \times 3.20 \mathrm{~m}$. Su volumen útil es de $18.7 \mathrm{~m}^{3}$.

La unidad de refrigeración consiste en un evaporador de techo INCON, que tiene una capacidad nominal de refrigeración de $3545 \mathrm{kcal} / \mathrm{h}$, y está provisto de dos ventiladores que provocan la circulación forzada del aire en el interior de la cámara. El circuito de refrigeración se completa con un compresor ACMARMETIC de 3 HP, con condensación por aire forzado. El refrigerante utilizado es R-22. Consta también de cuatro resistencias de desescarche, de $1100 \mathrm{~W}$ cada una. Con este equipo experimental se llevaron a cabo dos series de experiencias, en distintos momentos del desarrollo del presente trabajo, con diferentes estibas de alimentos congelados.

\subsubsection{ESTIBAS}

\subsection{TARIMA DE CAJAS}

La Figura 4.4 muestra una tarima estándar, de base rectangular de $1.00 \times 1.20$ $\mathrm{m}$ y con una estiba de $1.35 \mathrm{~m}$ de altura, colocada en el centro de la cámara de almacenamiento. Cada nivel del pallet consistía de cinco cajas de cartón cuya disposición se observa en la Figura. Las dimensiones de las cajas son las siguientes: $0.58 \mathrm{~m}$ de largo $\times 0.38 \mathrm{~m}$ de ancho $\times 0.15 \mathrm{~m}$ de altura. Este tipo de cajas es habitual mente usado para al macenar bloques de carne, cajas de hamburguesas, etc. En nuestras experiencias contenían bloques de carne vacuna picada, aproximadamente $33 \mathrm{~kg}$ de carne cada caja.

Para caracterizar el comportamiento térmico de la estiba se seleccionó una de las cajas ubicada en un vértice superior del pallet (una de las que se han sombreado en la Figura 4.4) ya que tienen una superficie mayor expuesta a la transferencia de calor con el ambiente de al macenamiento y por consiguiente serán las más afectadas por las fluctuaciones de temperatura del mismo. La Figura 4.5 muestra una de las cajas utilizadas indicando la ubicación de las termocuplas C1, C2, C3, C4 y C5. 


\subsection{ESTIBAS DE BALDES}

Se implementaron dos estibas tubulares empleando baldes plásticos cilíndricos, de 10 litros de capacidad, similares a los usados para el almacenamiento de frutillas y frambuesas congeladas IQF, cremas heladas, etc.

En la estiba A se colocaron 64 baldes en 4 niveles de 16 baldes cada uno, dispuestos en el centro de la cámara de almacenamiento. Cada balde fue colocado exactamente sobre un bal de inferior, como se muestra en la Figura 4.6. Los baldes se Ilenaron con 8 I de agua cada uno para simular una carga térmica equivalente a un balde lleno de fruta fina. Los baldes sombreados son los más expuestos a la transferencia de calor, en cada uno de ellos se colocaron dos termocuplas: una en el centro (A1) y otra en el borde externo superior (A2), como se indica en la Figura 4.7.

En la estiba B se colocaron 80 baldes dispuestos según muestra la Figura 4.8, sobre una base de madera. Esta estiba también se armó en el centro de la cámara; las paredes de la misma fueron cubiertas con una fila de baldes, de 4 unidades de altura. De este modo se intentó simular el comportamiento de una estiba almacenada en una cámara de mayores dimensiones.

Los baldes sellenaron con aproximadamente 8 I de agua cada uno, excepto 4 de ellos que contenían 10 I de helado comercial de vainilla. La Tabla siguienteindica la posición en la estiba de estos 4 baldes, y la ubicación de las termocuplas utilizadas.

Tabla 4.1. Posición de los baldes con helado y de las termocuplas en la estiba B.

\begin{tabular}{|ccccc||}
\hline Balde & Fila & Columna & Profundidad & Termocupla \\
\hline \hline A & 1 & 3 & 2 & B6 \\
B & 2 & 1 & 4 & B3y B7 \\
C & 3 & 1 & 1 & B5 \\
D & 4 & 3 & 3 & B2 y B4 \\
\hline \hline
\end{tabular}




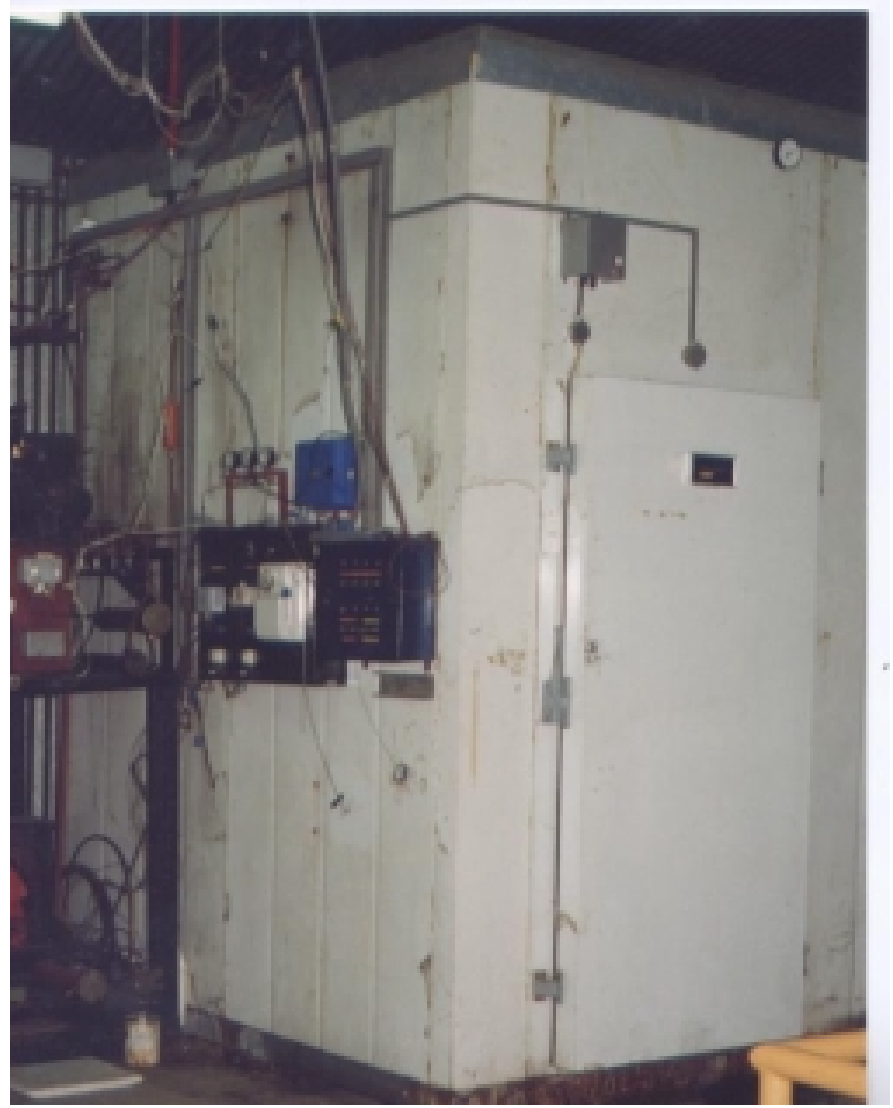

Figura 4.3. Cámara experimental de almacenamiento. 


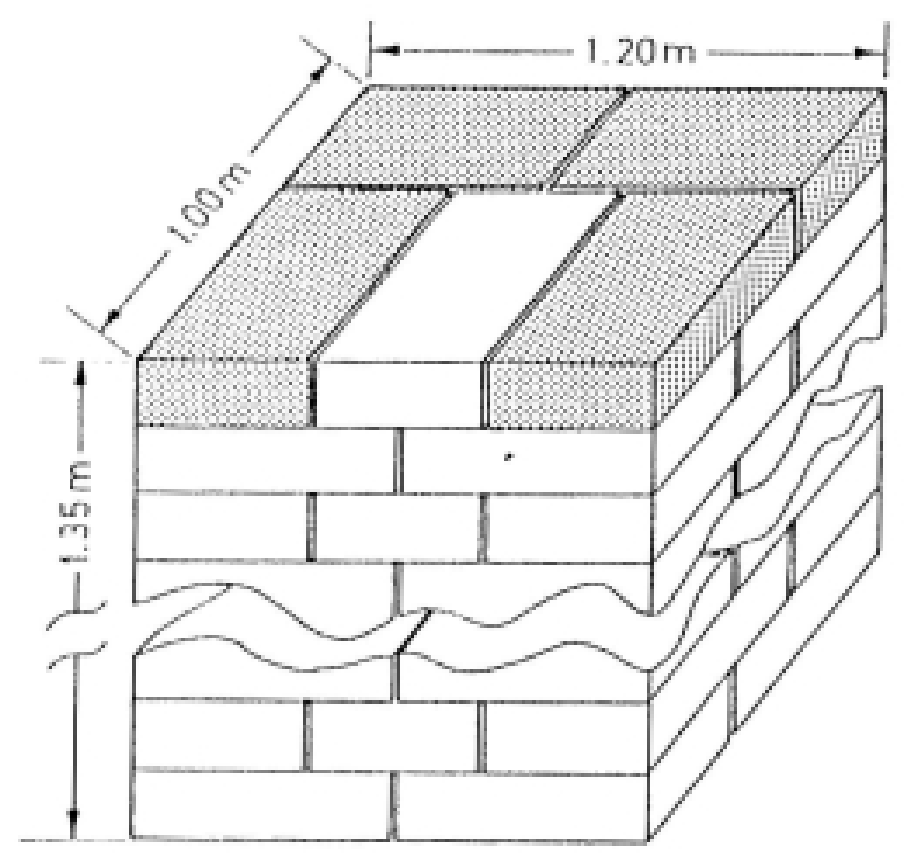

Fiqura 4.4. Tarima de caias.

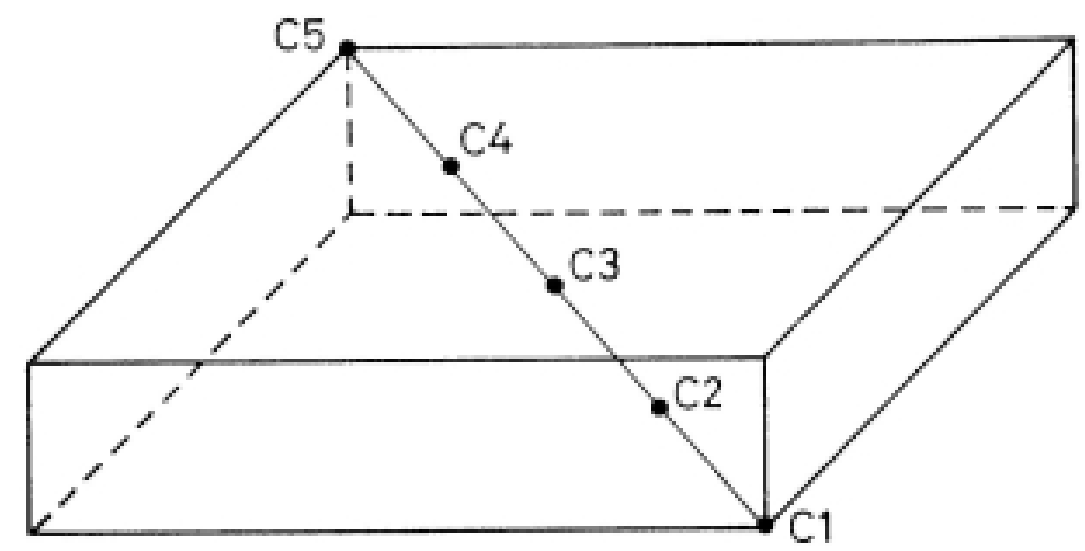

Figura 4.5. Caja de cartón indicando la posición de los puntos de medida. 


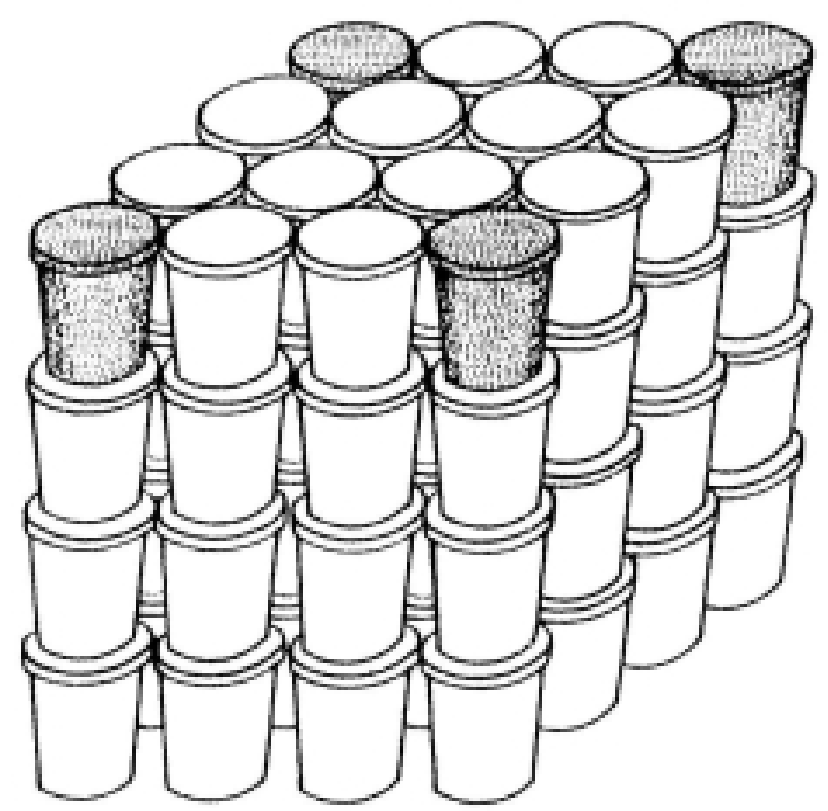

Figura 4.6. Estiba tubular de baldes A.

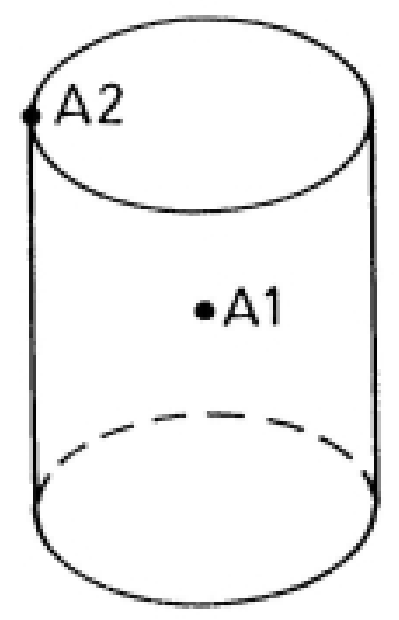

Figura 4.7. Balde plástico indicando la posición de las termocuplas A 1 y A2. 


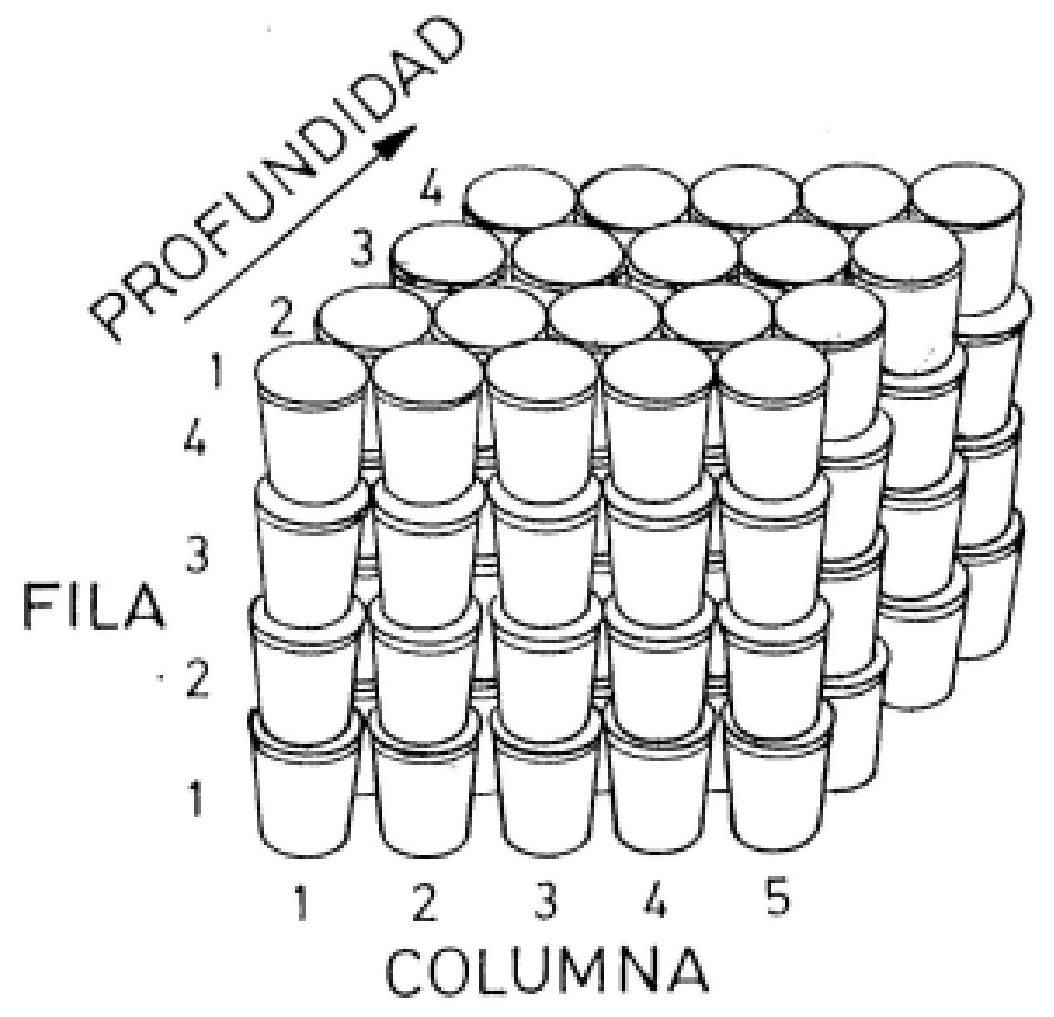

Figura 4.8. Estiba tubular de baldes B. 


\subsubsection{DESCRIPCION DE LAS MEDIDAS EXPERIMENTALES}

Para la primera serie de medidas experimentales, realizada con la tarima de cajas y con la estiba de baldes A, la cámara era controlada por un termostato estándar, cuyo objetivo es regular el funcionamiento del equipo de frío de modo tal que la temperatura del aire interno se mantenga dentro de un cierto rango, de varios grados Centígrados, fijado por el operador. Con este elemento de control poco refinado no fue posible obtener ninguna señal patrón, por ejemplo un escalón de temperatura constante. Se planteó entonces la necesidad de realizar un control manual de la operación a fin de obtener la respuesta del sistema a una señal patrón que fuera reproducible. En el inciso 4.2.1. se postuló que la mejor señal para obtener los coeficientes de la función de transferencia por la vía experimental era el pulso triangular porque, si su duración es muy pequeña comparada con el tiempo que tarda el sistema globalmente en responder, entonces el pulso de duración finita actúa como un impulso instantáneo. Por las dificultades de control fue imposible producir un pulso. La alternativa era provocar una perturbación escalón que, como veremos más adelante, nos da resultados que pueden ser transformados en la respuesta a un impulso (Levenspiel, 1976).

Para provocar el escalón se necesita además del elemento que genera frío, uno o varios elementos que provoquen aumento de la temperatura. Para ello se utilizaron las cuatro resistencias calefactoras enrolladas en los tubos del evaporador. Para lograr el salto térmico inicial del escalón se procedió a abrir la puerta de la cámara y provocar la mezcla del aire interior, a varios grados bajo cero, con el aire externo, a temperatura ambiente. En pocos segundos la temperatura del aire en el interior del ambiente de almacenamiento había ascendido a la temperatura deseada.

La Figura 4.9 muestra la respuesta del sistema (termocuplas A 1 y A2) a una señal escalón, producida con control manual. Inicialmente el sistema (estiba de baldes A) se

encontraba total mente uniformizado a $-32.7^{\circ} \mathrm{C}$; la temperatura promedio del aire es de $-14.3^{\circ} \mathrm{C}$.

Mediante un adquisidor de datos DATALOGGER FLUKE modelo $2240 \mathrm{C}$ se 
midió y registró la temperatura en función del tiempo en los cinco puntos de la caja indicados en la Figura 4.5 y en el caso de la estiba de baldes en las posiciones indicadas en la Figura 4.7. En ambos casos se registró la temperatura en varios puntos del aire en las inmediaciones de las correspondientes estibas de forma de tener información completa.

También se midieron experimental mente las respuestas de ambos sistemas a diferentes fluctuaciones en la temperatura del aire de la cámara para poder comparar estas respuestas experimentales con las respuestas predichas por las funciones de transferencia para las mismas condiciones ambientales. Para provocar estas perturbaciones, debido a las dificultades de control mencionadas, se trabajó con el termostato de la cámara, fijando un rango amplio de temperaturas de trabajo, de modo de reproducir los ciclos de enfriamiento-calentamiento habituales en todas las cámaras de al macenamiento. Las medidas realizadas se detallarán más adelante junto con los resultados obtenidos.

La segunda serie de experiencias fue realizada con la estiba de baldes B, utilizando un sistema de adquisición de datos y control modelo DAC500 de KEITHLEY. El sistema en sí es una estación de trabajo que mediante una tarjeta de entradas analógicas, específica para medir temperaturas (provista de compensación por junta fría), puede leer hasta 16 termocuplas simultáneamente. Las señales leídas son digitalizadas por un convertidor A/ D y transferidas mediante su interfase, a una PC AT. En ella las señales son procesadas mediante un programa, PROINTE (cuyo diagrama de bloques se describe en la Figura 4.10), desarrollado en lenguaje BASIC, que consiste en rutinas y comandos provistos con el equipo (SOFT500) que simplifican las tareas de entrada/ salida de señales analógicas y digitales, la generación de vectores y matrices de variables, la medida del tiempo, etc., y rutinas propias, particularmente las desarrolladas para llevar a cabo el control automático de la cámara frigorífica. La tarjeta dirigida a efectuar las acciones de control (PCM2) puede accionar distintos equipos eléctricos, a través de 12 relés montados sobre un rack de salida. En nuestro equipamiento, los relés actuaban sobre un elemento de refrigeración, el compresor de 3 HP ya mencionado; y en cinco elementos de cal efacción independientes: una válvula de solenoide que provoca el retorno de gas hacia el evaporador, disminuyendo la capacidad frigorífica del mismo, y las cuatro resistencias mencionadas en la primera disposición. Con este equipo de control fue 
posible producir señales pico mucho más precisas que con el control manual, trabajando a una alta velocidad de adquisición (1 s) y tomando acciones de control cada 5 s. Aún así, debido a la gran inercia térmica del sistema, y a la falta de elementos de calefacción y refrigeración adecuados para implementar un control más fino de las señales, fue necesario ejecutar una serie de corridas experimentales previas de modo de conocer adecuadamente el sistema y programar un algoritmo de control que lograse un buen seguimiento de la señal deseada, como puede observarse en la Figura 4.11. La Figura 4.12 muestra la respuesta de los puntos característicos de la estiba B a esta señal pico.

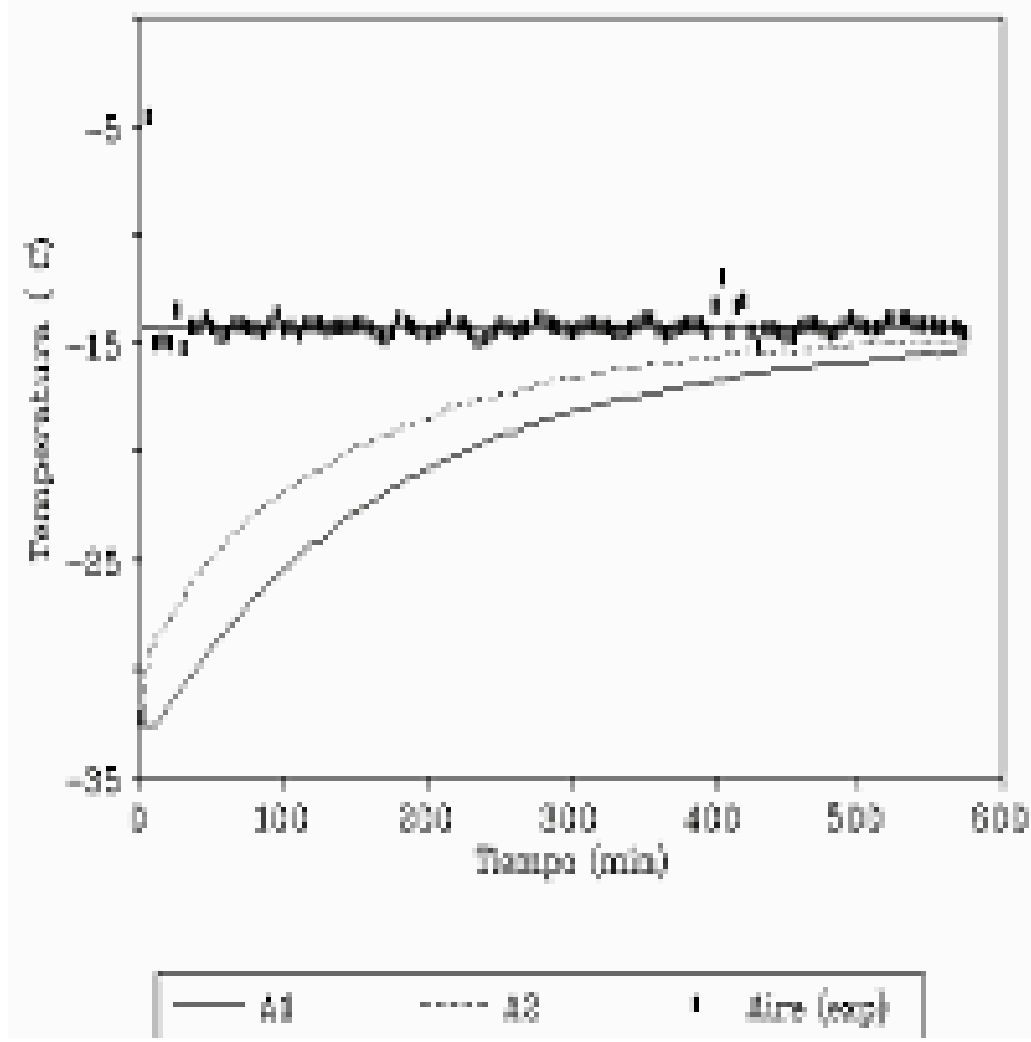

Figura 4.9. Señal escalón, obtenida con control manual. 


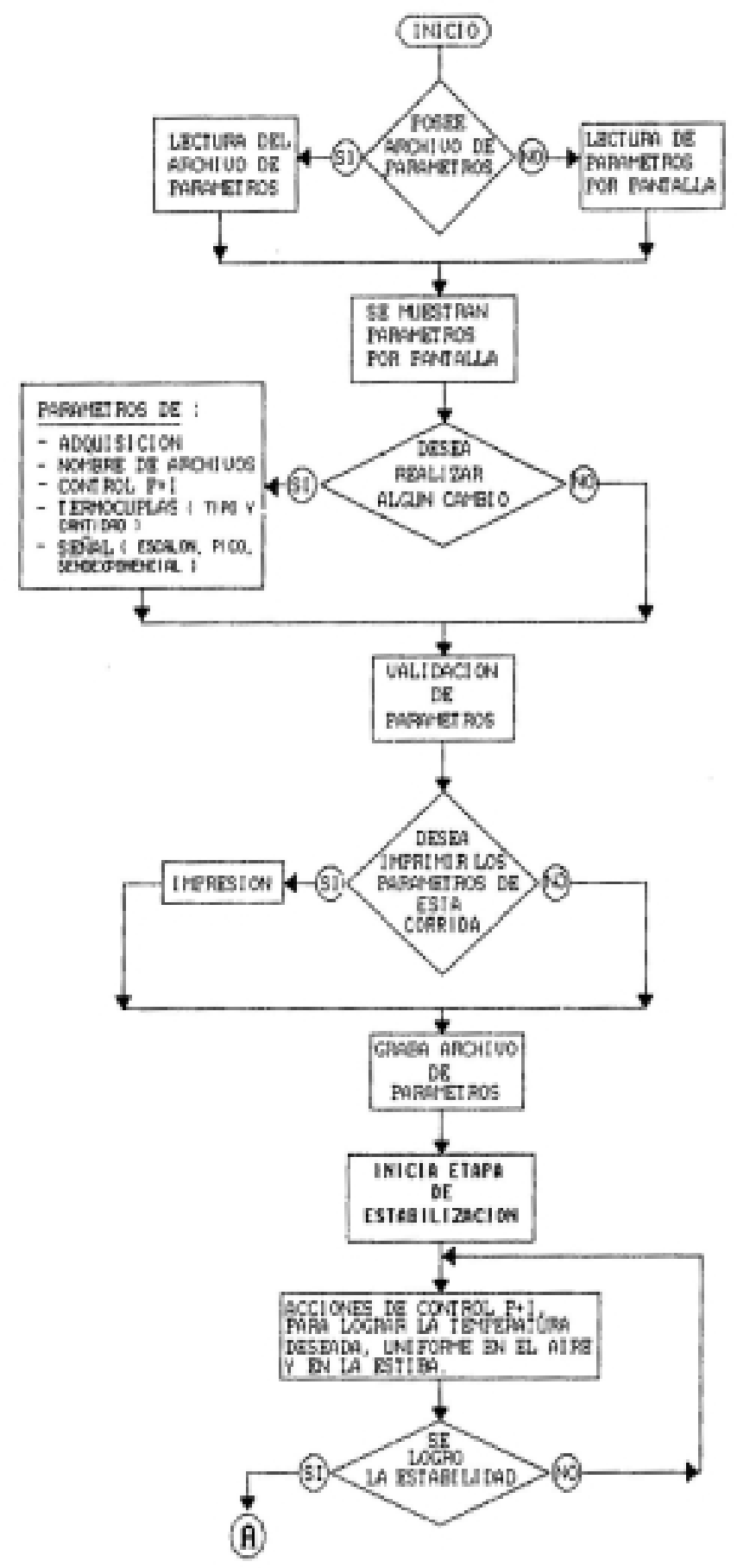

Figura 4.10. Diagrama en bloques del programa de adquisición de datos y control desarrollado. 


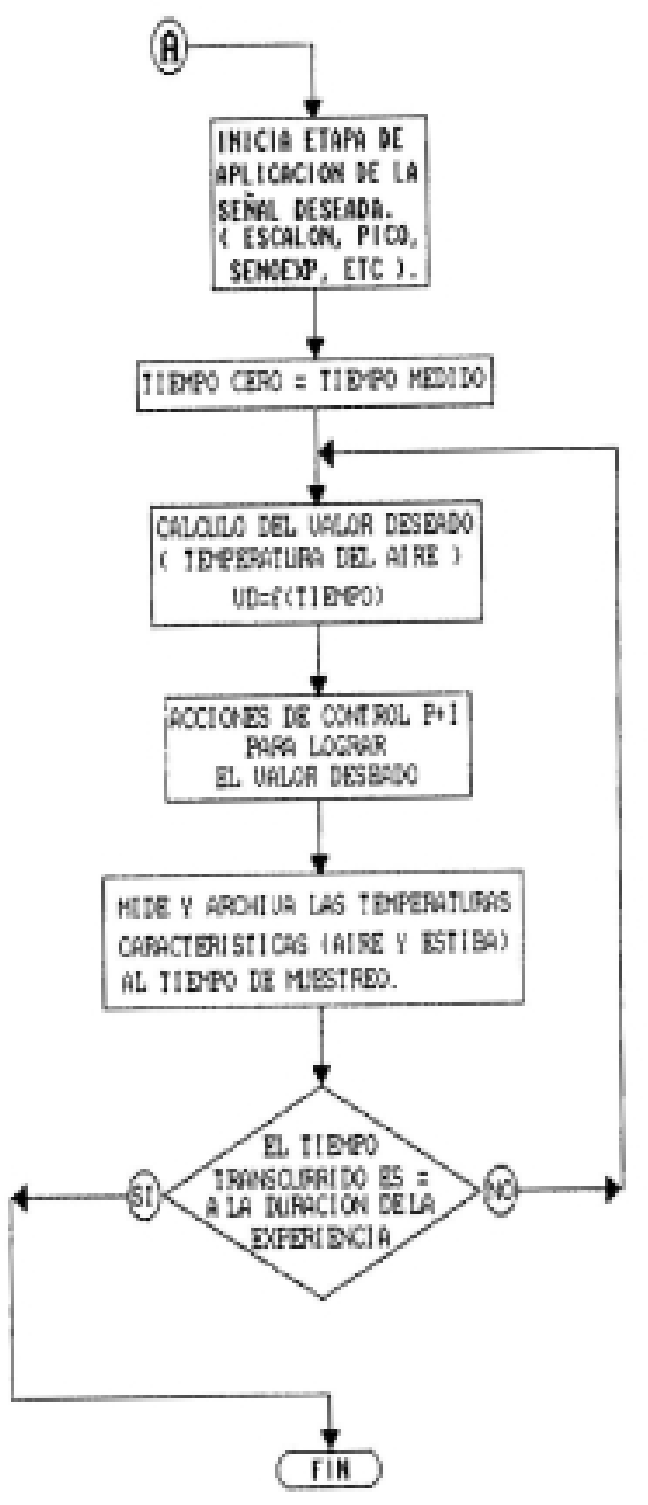

Figura 4.10. Continuación. 


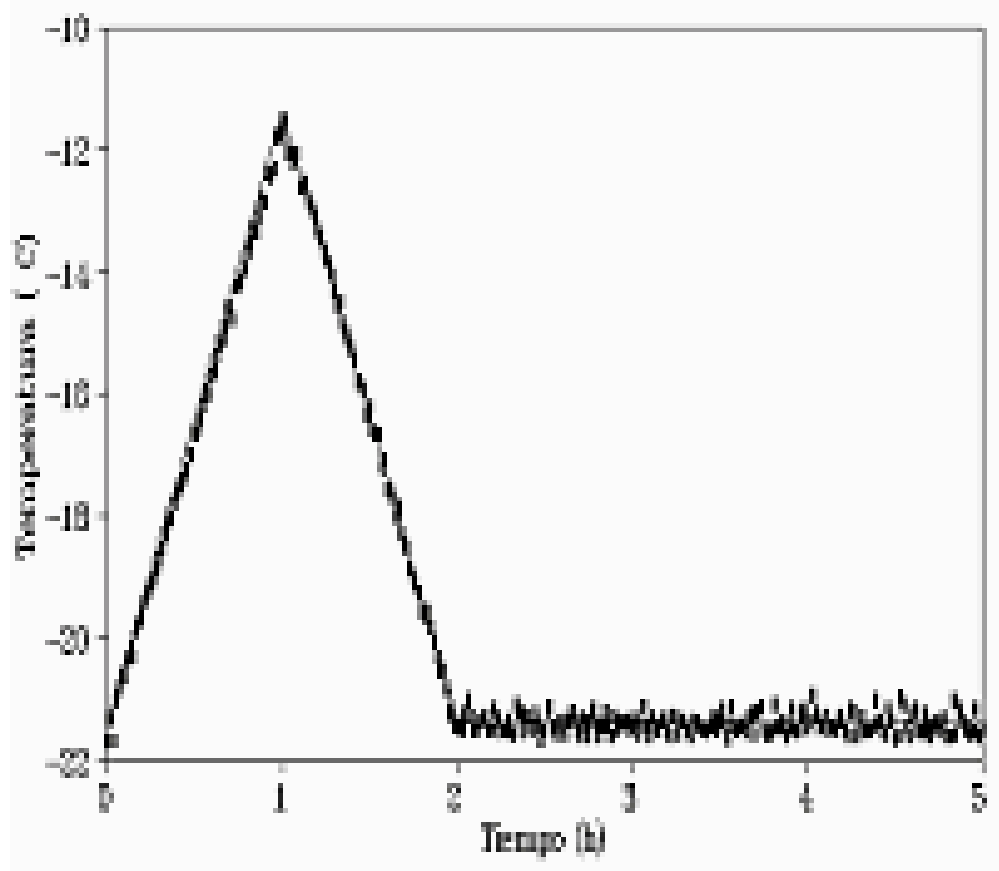

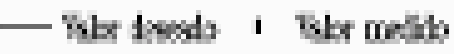

Figura 4.11. Señal pico, obtenida con control automático.

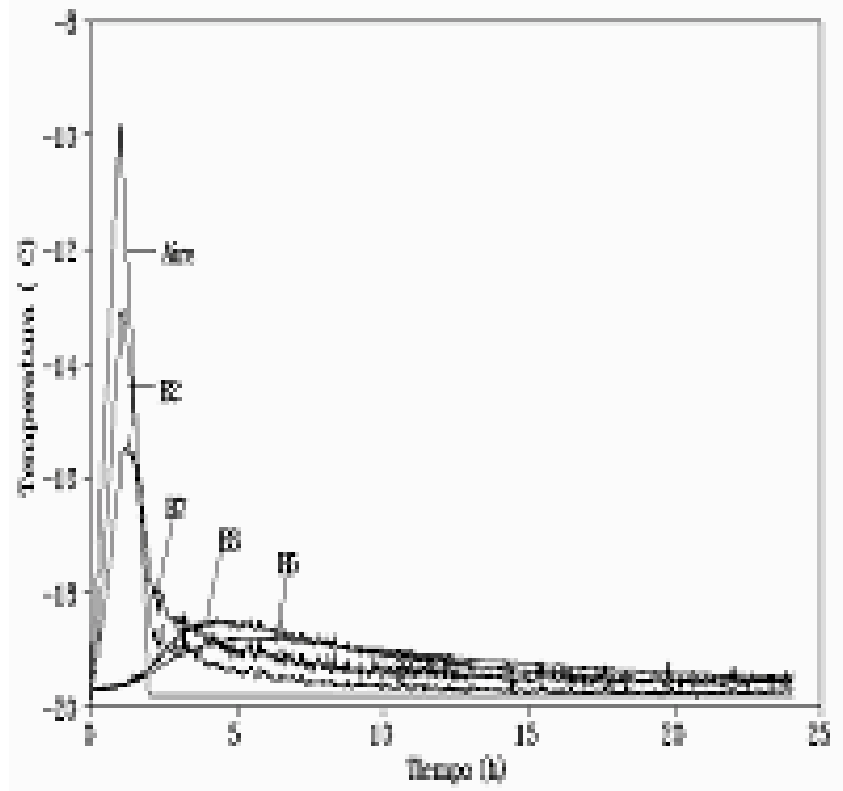

Figura 4.12. Respuesta de la estiba de baldes B a la señal pico. 


\subsubsection{CALCUlO DE LOS COEFICIENTES DE LA FUNCION DE TRAN SFEREN CIA}

\subsubsection{COEFICIENTES EXPERIMENTALES}

Como se ha mencionado anteriormente, para calcular los coeficientes $f_{n}$ por la vía experimental es necesario provocar una señal perturbación patrón y medir la respuesta térmica del sistema en varios puntos que se definirán como característicos del comportamiento global del mismo.

Teóricamente el mejor pulso de entrada sería el impulso o delta de Dirac (ec. (4.5)), pero en la práctica es imposible realizar un pulso de ancho cero y altura infinita. Si se provoca un pulso de ancho y altura finitas, deberá fijarse el ancho de acuerdo a la regla citada ( $\Delta \leq$ (T1/2) donde T1 es la menor constante de tiempo); el ancho tampoco puede ser muy pequeño ya que el sistema se perturbaría muy poco y resultaría muy difícil separar la señal de salida al pulso del ruido y del error experimental. La altura del pulso puede incrementarse pero no demasiado ya que no debe excederse la zona de linealidad (en los sistemas analizados en este trabajo suponemos alimentos congelados, por lo cual no debe trabajarse en la región de temperaturas donde se produce la mayor parte del cambio de fase, ya que deja de valer la suposición de que las propiedades termofísicas son independientes de la temperatura).

Si la señal patrón utilizada es un pico triangular el cálculo de los coeficientes es inmediato ya que la transformada $\mathrm{z}$ de la perturbación $\mathrm{P}(\mathrm{z})$ consta de un único término como indica la ec. (4.9). En este caso los coeficientes se calculan utilizando la ec. (4.15); esta metodología se empleó para calcular los coeficientes correspondientes a la estiba de baldes B. La Figura 4.12 muestra la respuesta de distintos puntos de la mencionada estiba a la señal pico.

$$
f_{n}=\frac{T((n+1) \Delta)-T_{i}}{I}
$$

Cuando la señal patrón de perturbación es un escalón, se puede construir la respuesta a un impulso derivando la respuesta al escalón. Llamando $E$ al tamaño del escalón $\left(E=T\right.$ aire $\left.-T_{i}\right)$ el impulso resultante tendrá una amplitud $I=E / \triangle$. La 
respuesta al impulso, $r(\mathrm{t})$, se genera derivando la respuesta experimental al escalón, $\mathrm{u}(\mathrm{t})$.

$$
r(t)=\frac{d u(t)}{d t}
$$

Como no se conocen todos los valores de la función $u(t)$ ni su expresión analítica, el cálculo de la derivada de la ec. (4.16) se aproxima por diferencias según (4.17):

$$
\left.r(n \Delta)=\frac{d u(t)}{d t}\right]_{t=n \Delta}=\frac{u((n+1) \Delta)-u((n-1) \Delta)}{2 \Delta}
$$

Si $u(n \Delta)=T(n \Delta)$ - Ti siendo $T(n \Delta)$ la temperatura en el punto de interés de la estiba en el tiempo $t=n \Delta, y$ reemplazamos en la ec. (4.10), resulta que los coeficientes dela función $F(z)$ quedan definidos por la ec. (4.18):

$$
f_{n}=\frac{T((n+1) \Delta)-T((n-1) \Delta)}{2\left(T_{\text {aire }}-T_{i}\right)}
$$

Con este esquema de cálculo, que identificamos como "Método A" en los resultados graficados, se calcularon los coeficientes $f_{n}$ correspondientes a los puntos representativos del pallet de cajas y de la estiba tubular de baldes $A$.

El cálculo de estos coeficientes presenta dos fuentes de error: uno es la aproximación al calcular una derivada por un cociente de diferencias, el otro error es que el escalón logrado no era perfecto ya que la temperatura del aire no se mantuvo constante. Para corregir estos errores se calcularon los coeficientes $f_{n}$ efectuando $\mathrm{A}$ cociente entre los polinomios $\mathrm{R}(\mathrm{z})$ y $\mathrm{P}(\mathrm{z})$ siendo:

$$
r(n \Delta)=T(n \Delta)-T_{i} \quad y \quad p(n \Delta)=T_{\text {aire }}-T_{i}
$$

El cociente de polinomios se calculó utilizando un algoritmo de bibliografía (Luyben, 1973), en los resultados graficados este método de cálculo se ha identificado como "Método B". 


\subsubsection{COEFICIENTES NUMERICOS}

Cuando la geometría del sistema es sencilla, es posible modelar su comportamiento mediante un esquema numérico de diferencias finitas, siempre que se conozcan las propiedades físicas y demás parámetros del balance diferencial de energía.

Este análisis se realizó con la estiba de cajas. No se intentó con las estibas de baldes ya que al no ser una estiba compacta los huecos que quedan entre los distintos baldes hacen muy difícil su modelización.

Para calcular los coeficientes se resolvió el siguiente balance:

$$
\frac{\partial T}{\partial t}=\alpha \Delta^{2} T
$$

considerando transferencia de energía en las tres direcciones ortogonales $x, y, z$ y con las siguientes condiciones inicial y de contorno:

$$
\begin{array}{ccccc}
T=T_{i} \quad V E E x & V E E y & V E E z & t=0 \\
-k \frac{\partial T}{\partial x}=h_{x}\left(T-T_{\text {aire }}\right) & x=L_{x} ; & \frac{\partial T}{\partial x}=0 & x={ }_{-} L_{x} & t>0 \\
-k \frac{\partial T}{\partial y}=h_{y}\left(T-T_{\text {aire }}\right) & y=L_{y} ; & \frac{\partial T}{\partial y}=0 & y=L_{-} L_{y} & t>0 \\
-k \frac{\partial T}{\partial z}=h_{z}\left(T-T_{\text {aire }}\right) & z=L_{z} ; & \frac{\partial T}{\partial z}=0 & z=L_{-} L_{z} & t>0
\end{array}
$$

El origen de coordenadas coincide con el centro geométrico de la caja, siendo $L_{x}$, $L_{y}$ y $L_{z}$ las correspondientes semilongitudes de los ejes principales; $h_{x}, h_{y}$ y $h_{z}$ son los respectivos coeficientes de transferencia calórica. La temperatura del aire (señal pico) varía de acuerdo a la siguiente expresión: 


$$
T_{\text {aire }}=\left\{\begin{array}{lc}
T_{i} & t \leq 0 y t \geq 2 \Delta \\
T_{i}+\frac{I}{\Delta} t & 0 \leq t \leq \Delta \\
T_{i}+2 I-\frac{I}{\Delta} t & \Delta \leq t \leq 2 \Delta
\end{array}\right.
$$

El balance fue resuelto con un esquema numérico de diferencias finitas explícito, implementado en lenguaje FORTRAN 77, utilizando una grilla de $13 \times 9 \times$ 9 puntos y un intervalo de tiempo de $30 \mathrm{~s}$. Para las cajas era $L_{x}=0.29 \mathrm{~m}, L_{y}=0.19 \mathrm{~m}$ y $\mathrm{Lz}=0.075 \mathrm{~m}$. Los coeficientes de transferencia calórica globales se calcularon a partir de la medida de la velocidad del aire en las inmediaciones de la caja a simular y de las características del cartón de la misma, resultando $h_{x}=h_{y}=3.5 \mathrm{~W} /\left(\mathrm{m}^{20} \mathrm{C}\right)$ y $h_{z}=5.0 \mathrm{~W} /\left(\mathrm{m}^{20} \mathrm{C}\right)$.

Los coeficientes $f_{n}$ en los 5 puntos representativos de la caja fueron calculados con la ec. (4.15). En las Figuras, este método de cál culo se ha identificado como "Método C".

\subsubsection{COMPARACION ENTRE COEFICIENTES NUMERICOS $Y$ EXPERIMENTALES}

En la Figura 4.13 se han graficado los coeficientes del centro de la caja (C3) calculados por las distintas metodologías analizadas. Análogamente la Figura 4.14 muestra los coeficientes correspondientes a la posición C5. En ambos casos se observa que los coeficientes obtenidos por ambos métodos experimentales son muy fluctuantes, oscilando alrededor del valor obtenido por el modelo numérico. La dispersión de los coeficientes

experimentales se atribuye a las dificultades en el control y adquisición de los datos de temperatura. Otra fuente de error se origina en que, excepto para los primeros coeficientes, en los siguientes el valor absoluto del coeficiente es del mismo orden que el dela fluctuación. 
En las Figuras 4.15 y 4.16 se han graficado los coeficientes experimentales correspondientes a los dos puntos característicos de la estiba de bal des $A$.

Al comparar los métodos de cálculo A y B se observa que en ambas estibas el primer coeficiente del punto más perturbado (el vértice) resulta mayor calculado por el método B. Este resultado influye en las respuestas térmicas predichas ya que ese coeficiente tiene mucho más peso que los siguientes.

En la Figura 4.17 se han graficado los coeficientes experimentales de distintos puntos de la estiba de baldes B, obtenidos de la respuesta a la señal pico. En este caso se observa que el tipo de respuesta es similar a la que puede obtenerse con un modelo numérico, en mayor medida gracias a que experimentalmente se logró un buen seguimiento de la señal deseada.

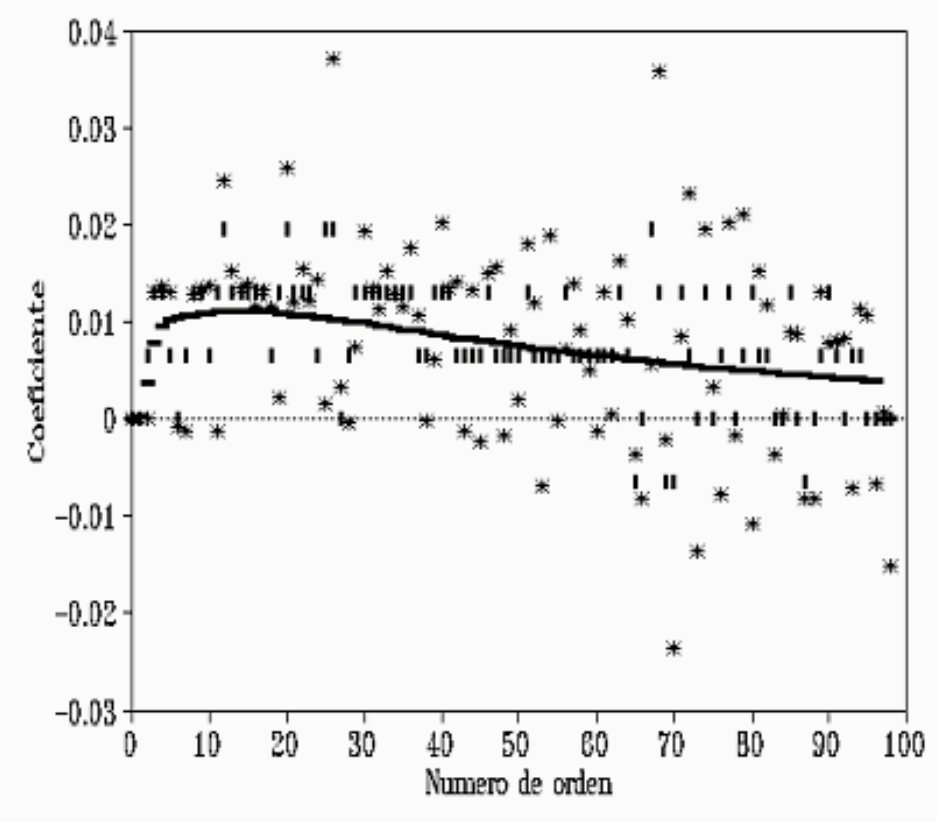

I Metodo A * Metodo B - Metodo C

Figura 4.13. Coeficientes $f_{n}$ correspondientes al punto C3. 


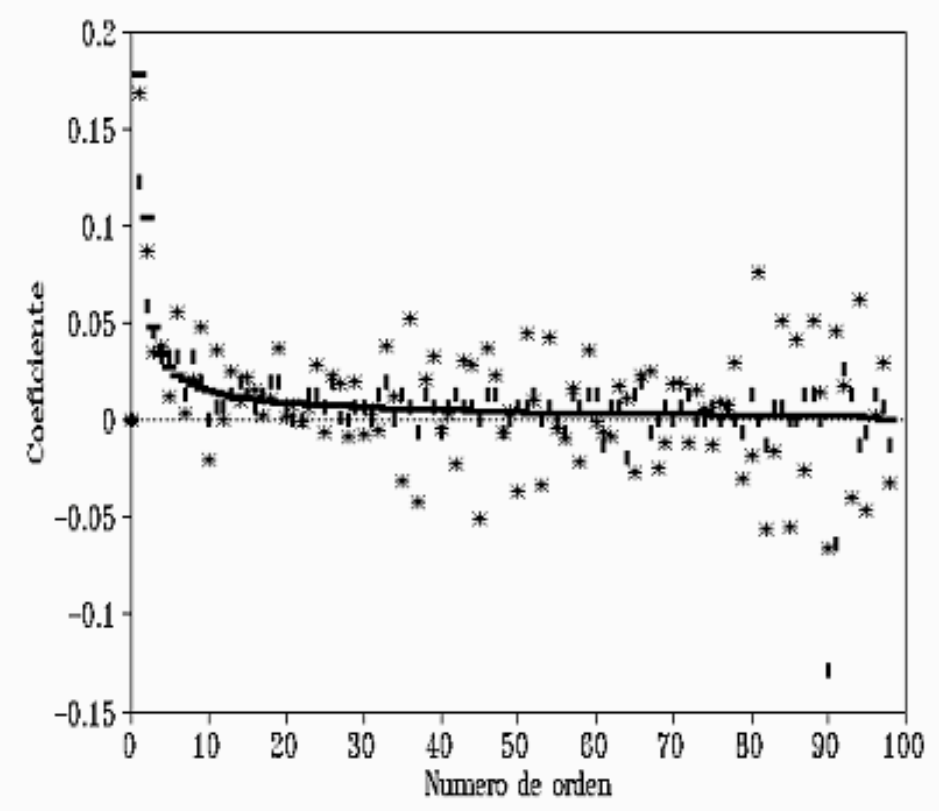

I Metodo A * Metodo B - Metodo C

Figura 4.14. Coeficientes $f_{n}$ correspondientes al punto $C 5$.

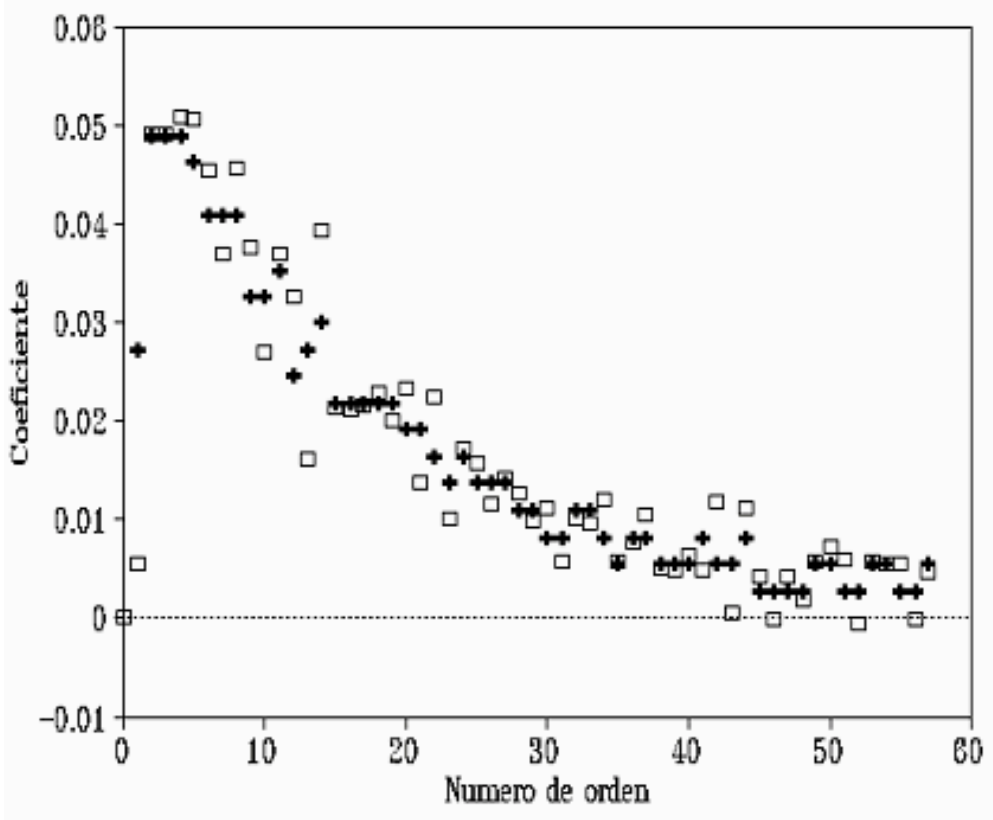

+ Metodo A $\square$ Metodo B

Figura 4.15. Coeficientes $f_{n}$ correspondientes al punto $A 1$. 


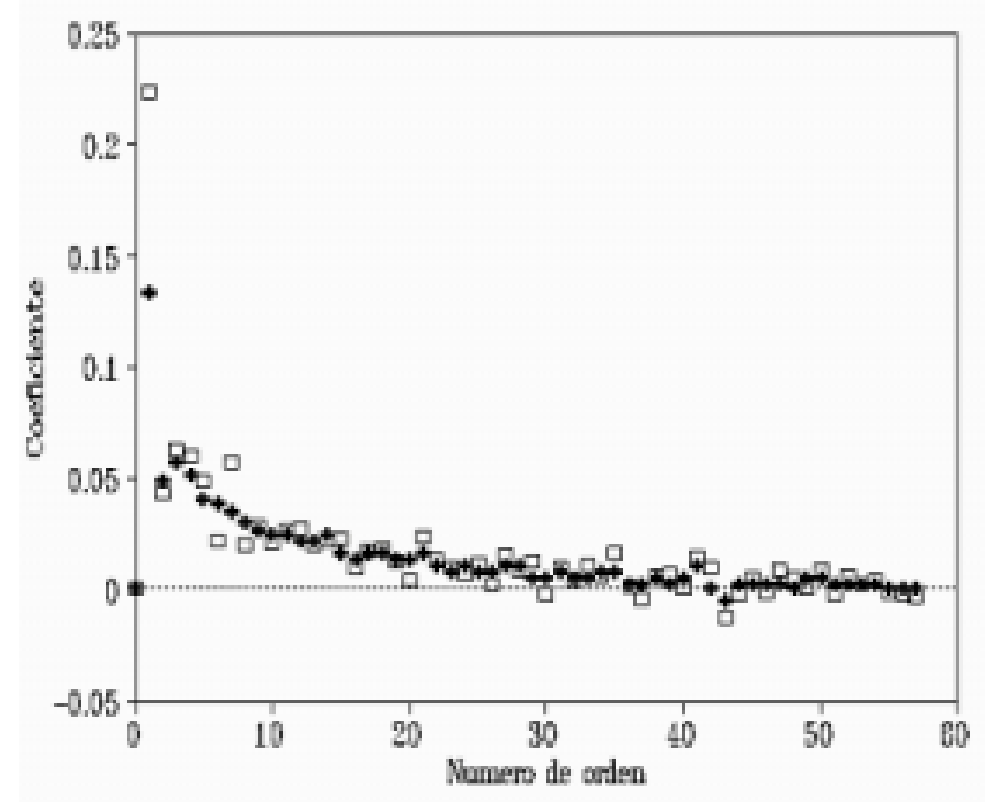

- Netodo $A$ D Hetodo B

Fiqura 4.16. Coeficientes $f_{n}$ correspondientes al punto $A 2$.

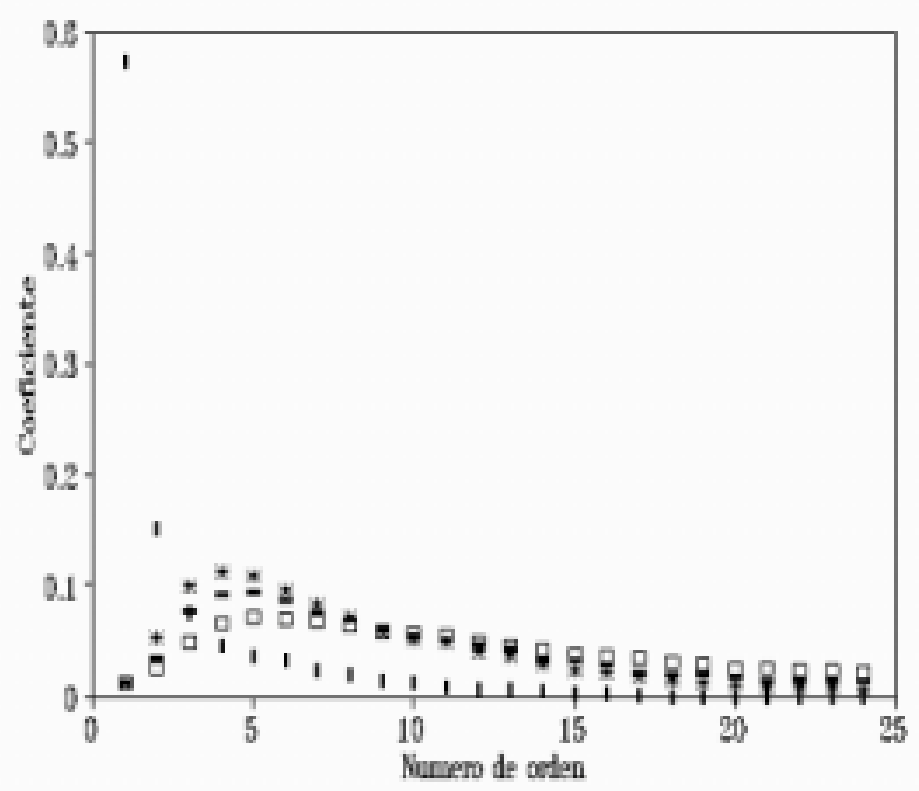

$$
\text { 1 } 8 \text { - }
$$

Figura 4.17. Coeficientes $f_{n}$ correspondientes a distintos puntos representativos de la estiba de baldes B. 


\subsubsection{RESULTADOS: PREDICCION DE RESPUESTAS TERMICAS FRENTE A DISTINTAS PERTURBACIONES}

En la Figura 4.18 se han graficado la temperatura del aire, y las temperaturas medidas en las posiciones C 3 y C5 de la caja del pallet seleccionada, cuando se provoca una perturbación del tipo seno-exponencial en la temperatura del ambiente dealmacenamiento.

Para validar la metodología de cálculo propuesta, estas historias térmicas experimentales fueron comparadas con las correspondientes historias predichas con los distintos juegos de coeficientes $\mathrm{fn}_{\mathrm{n}}$ cal culados.

En la Figura 4.19 se compara la respuesta experimental del centro de la caja (C3) con las respuestas calculadas por los tres métodos de cálculo antes descriptos. Análogamente la Figura 4.20 compara las respuestas experimental y calculada correspondientes al vértice externo superior de la caja (C5). La precisión obtenida con los coeficientes calculados por los métodos $\mathrm{B}$ y $\mathrm{C}$ son comparables, el método $\mathrm{A}$ como habíamos adelantado es menos preciso al predecir la respuesta térmica del punto $\mathrm{C} 5$.

También se analizó la influencia del intervalo de muestreo $\Delta$ en la precisión de las historias térmicas predichas. La Figura 4.21 compara la respuesta experimental del punto $\mathrm{C} 3$ con las respuestas calculadas utilizando tres intervalos de muestreo: 15, 30 y 45 minutos respectivamente. La Figura 4.22 a su vez, permite comparar las distintas respuestas obtenidas con los tres intervalos de muestreo, correspondientes al punto $\mathrm{C} 5$ de la caja. Se observa que a medida que se incrementa el valor de $\Delta$ las respuestas calculadas son menos precisas, en el caso de los 45 minutos es aproximadamente un $1 / 2$ del período de la señal de perturbación.

En la Figura 4.23 se comparan los resultados obtenidos al calcular las respuestas térmicas del punto $\mathrm{C} 5$ con distinto valor de $\mathrm{N}$. Se observa que al trabajar con pocos coeficientes se comete un error apreciable ya que se desprecia parcialmente la influencia de la señal de entrada.

Un análisis similar se realizó con los resultados obtenidos para la estiba de 
baldes A, con la salvedad de que no se cuenta con resultados numéricos.

En la Figura 4.24 se ha graficado el comportamiento experimental de los dos puntos característicos de la estiba frente a una fluctuación externa del tipo senoexponencial. Las Figuras 4.25 y 4.26 comparan los resultados obtenidos para los puntos $A 1$ y $A 2$ utilizando los dos juegos de coeficientes $f_{n}$ experimentales. En ambos puntos se obtienen mejores resultados con los coeficientes calculados mediante el cociente de polinomios, ya que esta metodología de cálculo de la función de transferencia contempla fielmente la señal experimental de perturbación (método B). Por ejemplo, la sumatoria de coeficientes del punto $A 2$, según $\mathrm{el}$ método A, vale sólo 0.835 , que es mucho menor que el valor límite de 0.99 que se impuso como criterio de selección del número de coeficientes.

En las Figuras 4.27 y 4.28 se han graficado las respuestas térmicas experimentales y calculadas de los puntos A 1 y A2, utilizando diferentes valores del intervalo de muestreo $\Delta$. Nuevamente se aprecia que la precisión de las temperaturas predichas aumenta a medida que disminuyeel intervalo de muestreo.

La Figura 4.29 muestra la historia térmica experimental del punto A2 como así también las respuestas predichas utilizando 20 y 40 términos para representar $\mathrm{F}(\mathrm{z})$. Vuelve a comprobarse que el valor de $\mathrm{N}$ afecta la precisión de las respuestas térmicas calculadas.

En cuanto a la estiba de baldes B, experimentalmente se midió la evolución de la temperatura en los distintos puntos indicados en la tabla 4.1. Dichas historias térmicas se compararon con las respuestas térmicas calculadas con los coeficientes experimentales graficados en la Figura 4.17, en todos los cálcul os se han utilizado 24 coeficientes.

En la Figura 4.30 se muestra la temperatura de los puntos B2 y B3 cuando se provoca una perturbación escalón.

En la Figura 4.31 se han graficado las respuestas, experimental y calculada, del punto B2 frente a una perturbación seno-exponencial. Análogamente en la Figura 4.32 se han graficado las respuestas del punto B5 frente a la misma perturbación externa. 


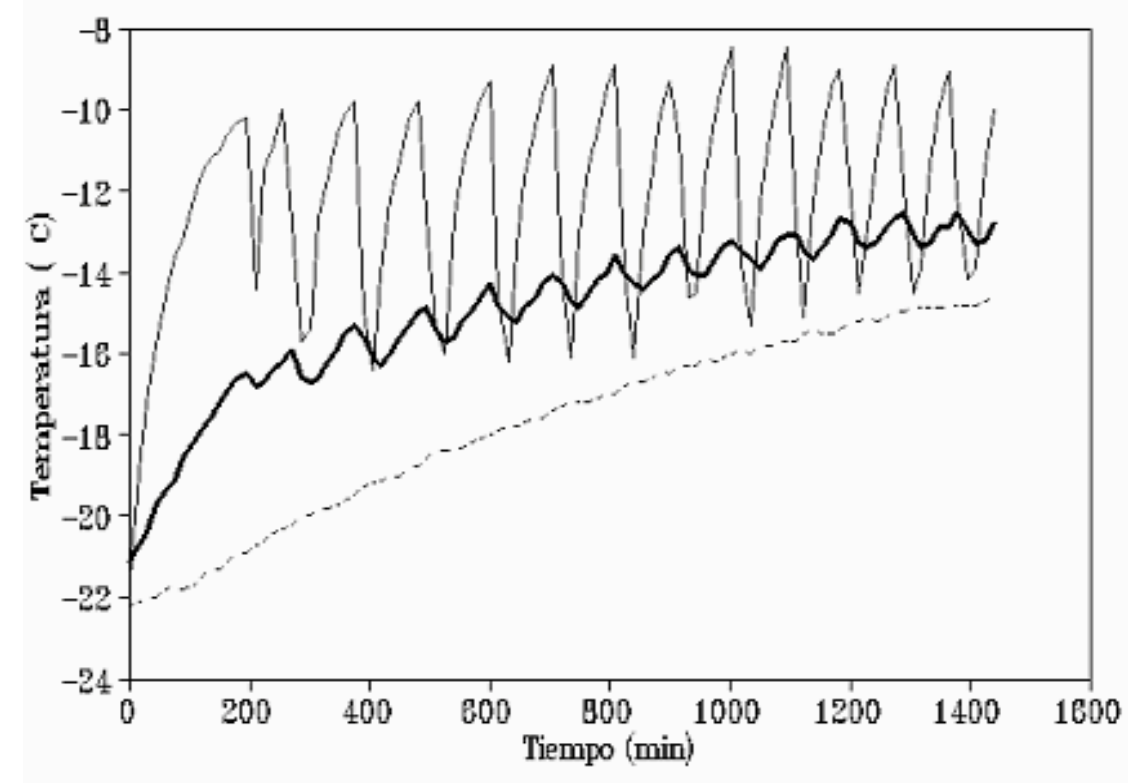

$$
\text { --- } \mathrm{C3}-\mathrm{C5}-\mathrm{Aire}
$$

Figura 4.18. Tarima de cajas. Respuesta experimental frente a una perturbación en la temperatura del aire

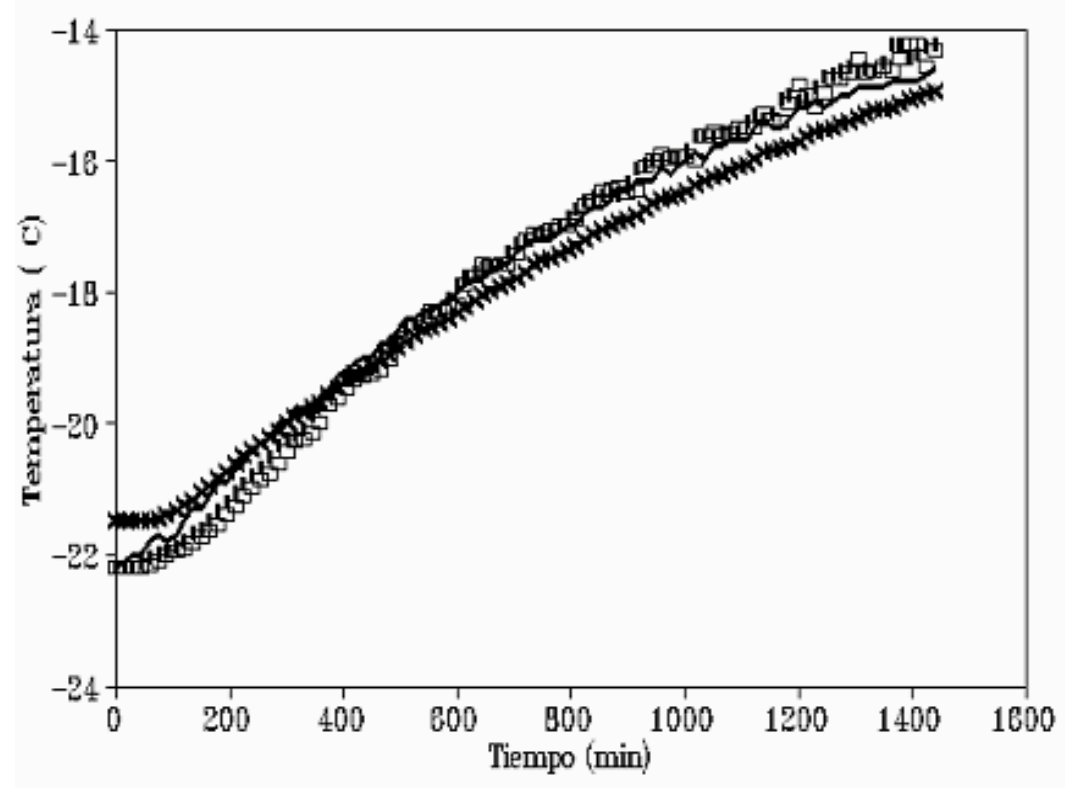

Experimen I Metodo A $\square$ Metodo B $\times$ Metodo C

Figura 4.19. Respuestas térmicas, experimental y calculadas, correspondientes al punto C3. 


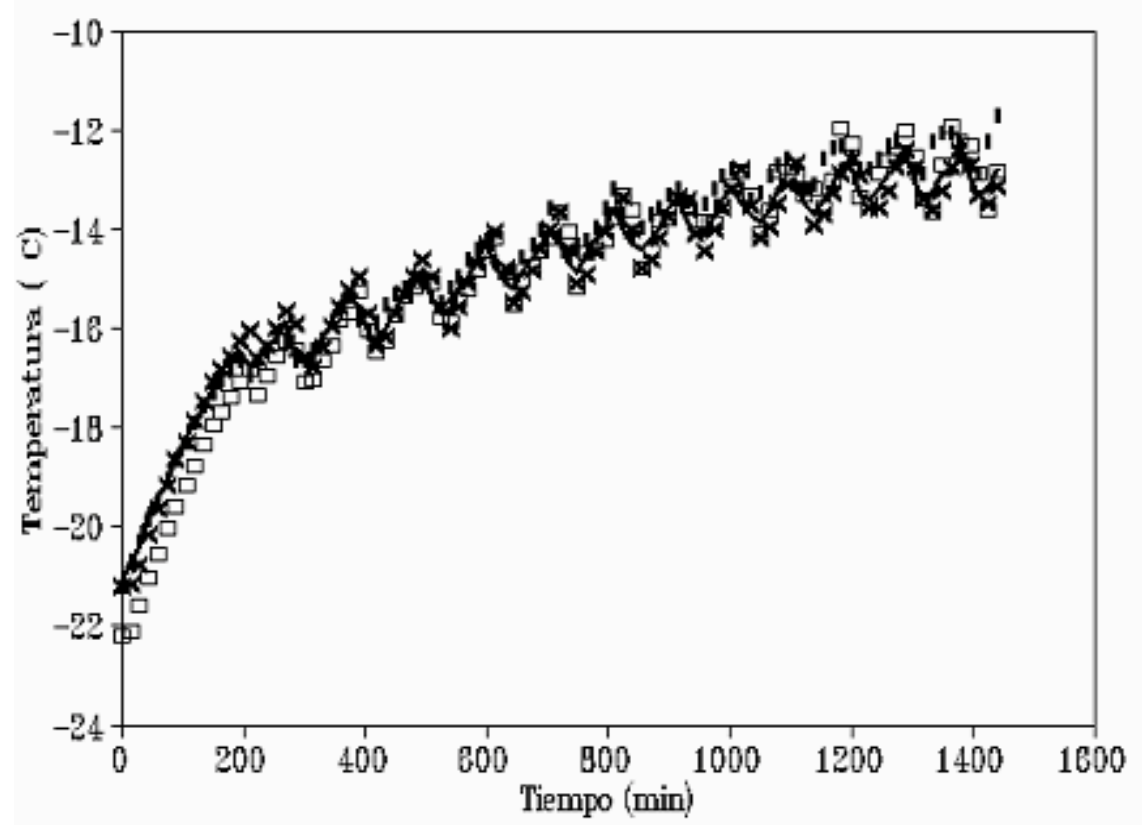

- Exper. I Metodo A $\square$ Metodo B $\times$ Metodo C

Figura 4.20. Respuestas térmicas, experimental y calculadas, correspondientes al punto $\mathrm{C} 5$.

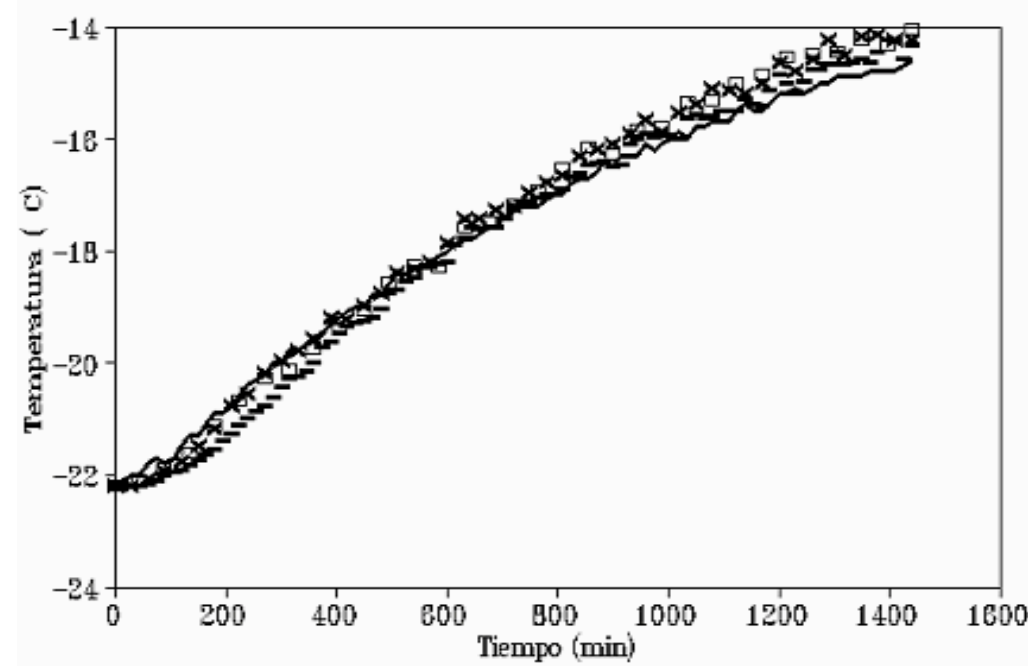

— Esper. $\quad-\quad=15 \min \times \quad=30 \min \quad \square \quad=45 \min$

Figura 4.21. Respuesta térmica, experimental y cal culadas por el método B, con distintos intervalos de muestreo, correspondientes al punto C3. 


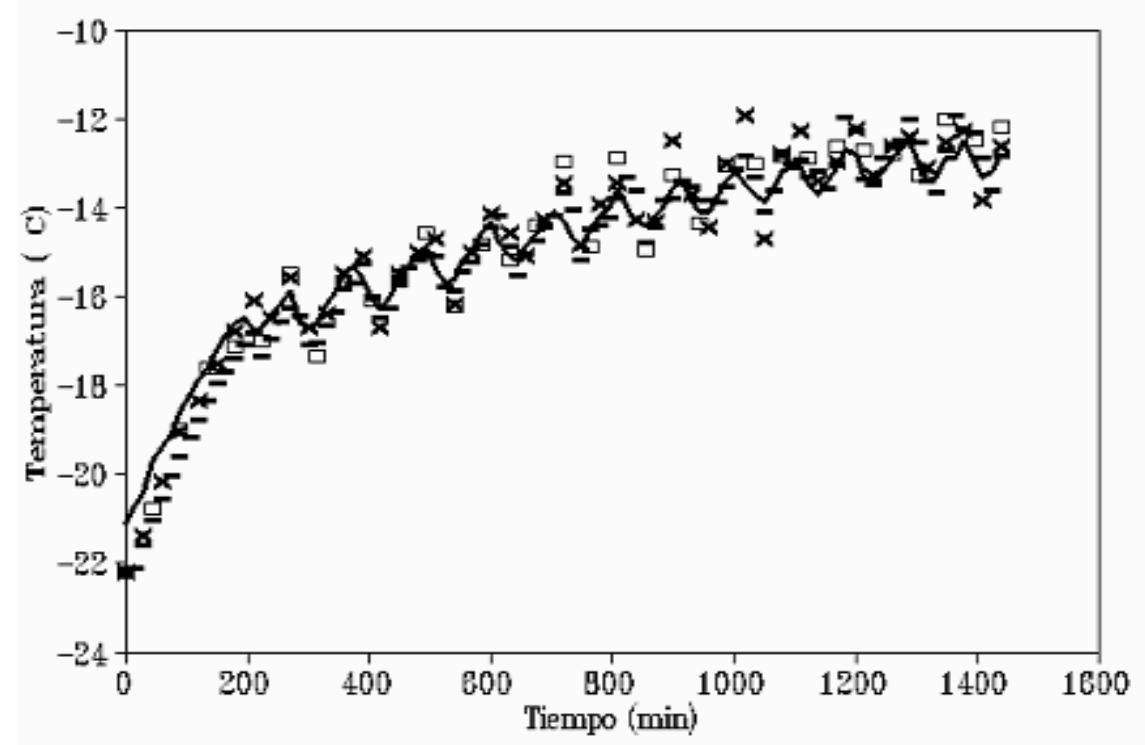

- Exper. - $=15 \mathrm{~min} \times=30 \mathrm{~min}$ 口 $=45 \mathrm{~min}$

Figura 4.22. Respuesta térmica, experimental y cal culadas por el método $\mathrm{B}$, con distintos intervalos de muestreo, correspondientes al punto $\mathrm{C} 5$.
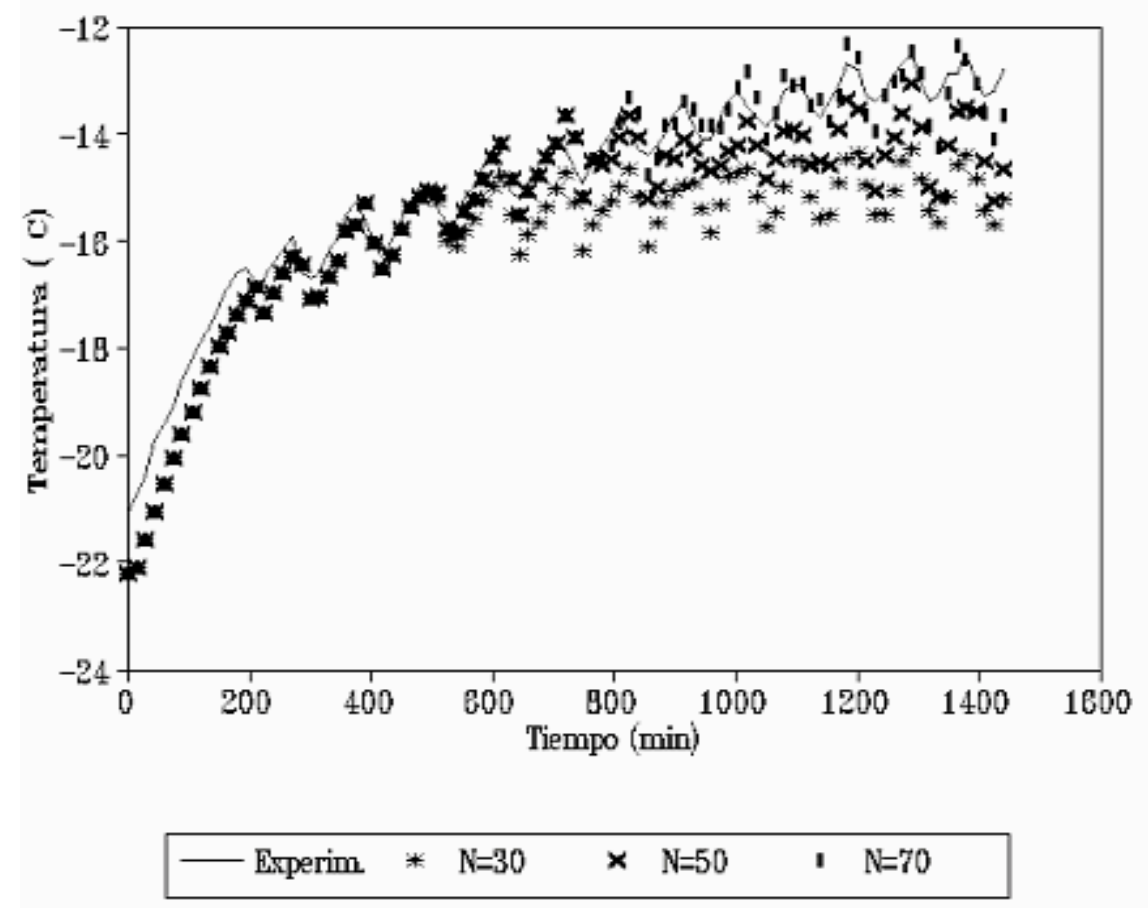

Figura 4.23. Respuesta térmica del punto $\mathrm{C} 5$ de la tarima de cajas, calculada con distintos valores de N. 


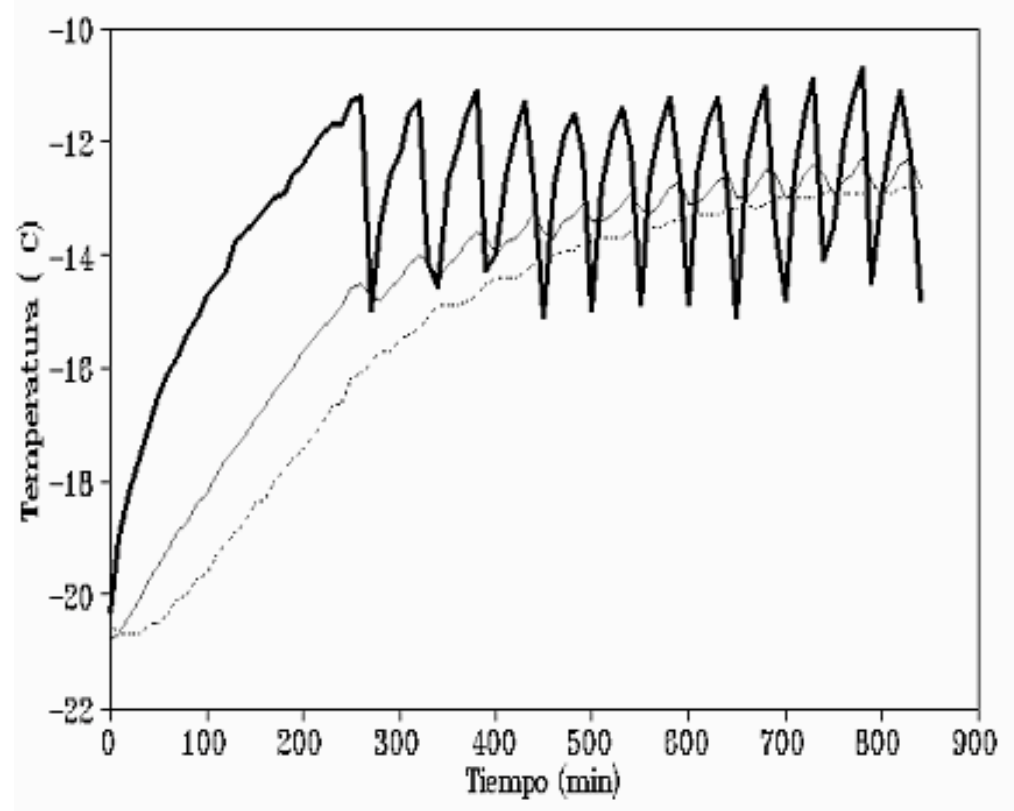

…… $\mathrm{Al}-\mathrm{A}-\mathrm{Aire}$

Figura 4.24. Respuestas térmicas de los dos puntos característicos de la esti ba de baldes, frente a una perturbación seno-exponencial.

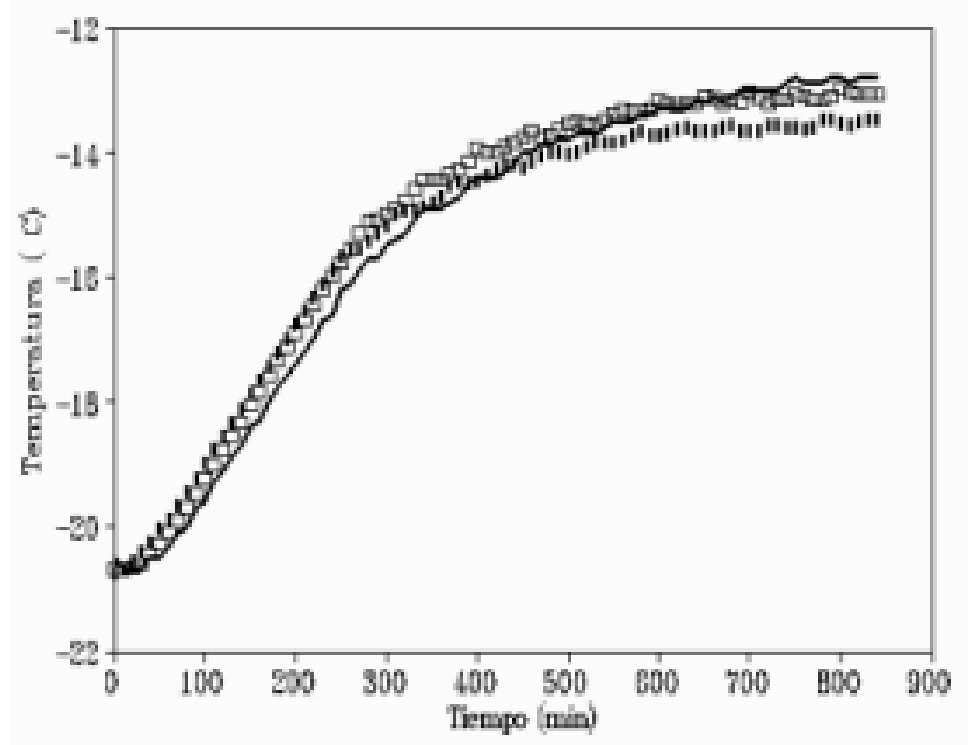

Reperimental I Metodo A a Netodo B

Figura 4.25. Respuestas térmicas, experimental y calculadas por distintos métodos, correspondientes al punto A 1 , de la estiba de baldes $A$. 


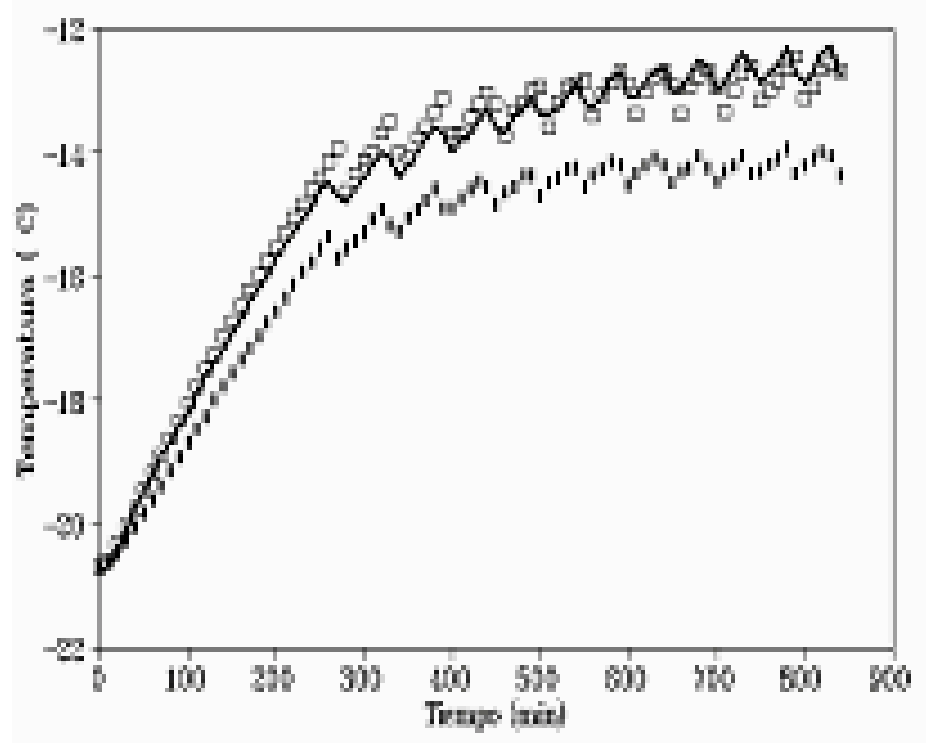

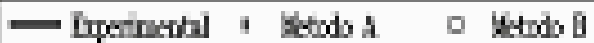

Figura 4.26. Respuestas térmicas, experimental y calculadas por distintos métodos, correspondientes al punto $\mathrm{A} 2$, de la estiba de baldes $\mathrm{A}$.

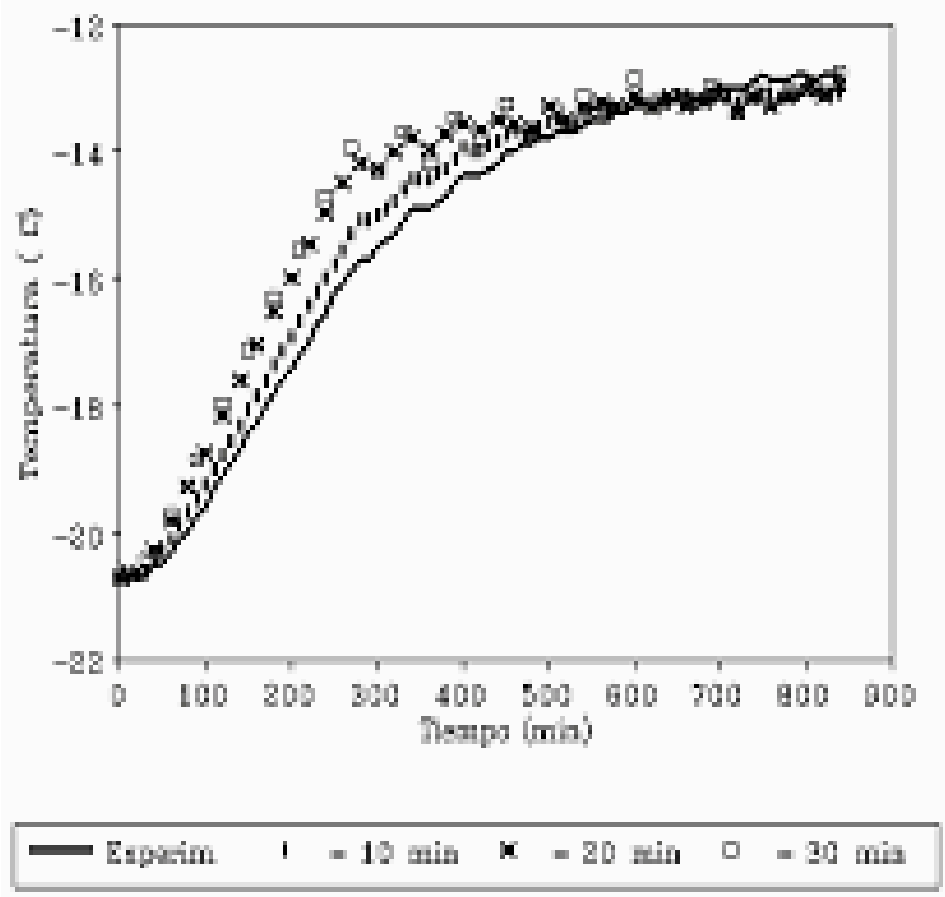

Figura 4.27. Respuestas térmicas, experimental y calculadas con diferentes interval os de muestreo, correspondientes al punto $\mathrm{A} 1$. 


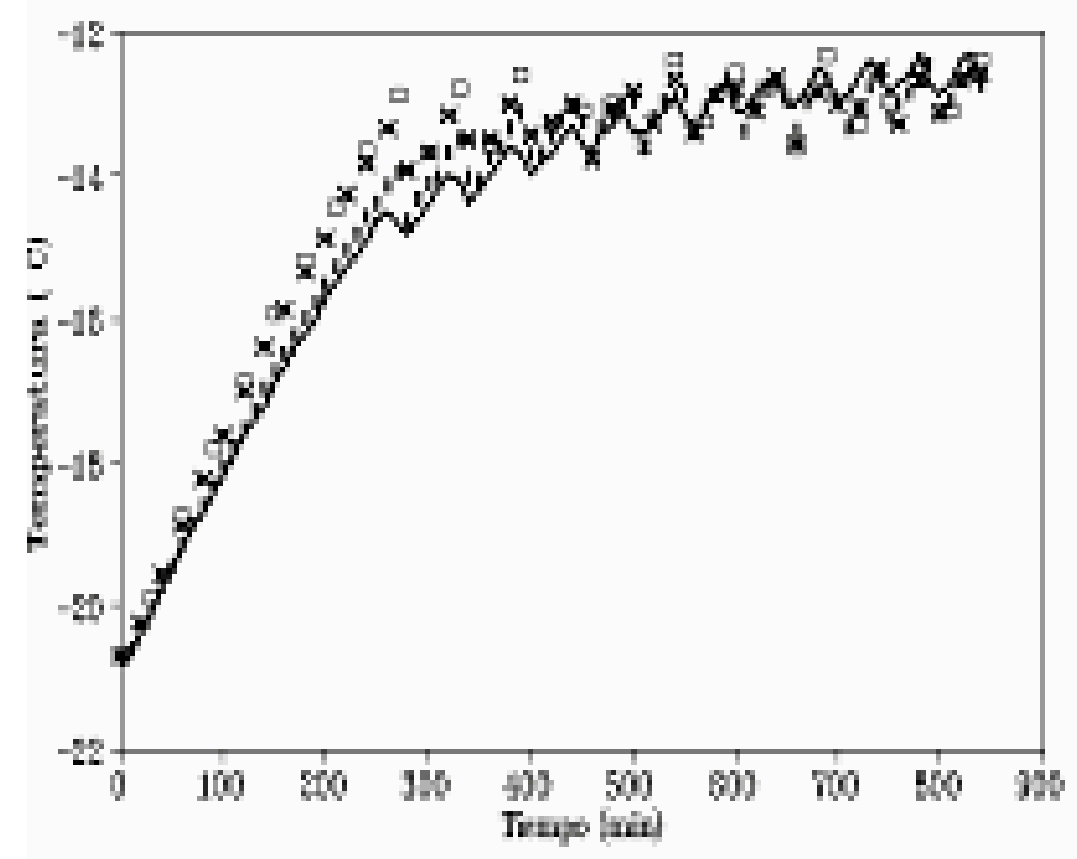

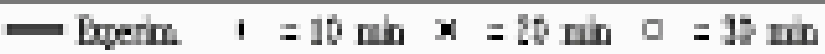

Figura 4.28. Respuestas térmicas, experimental y calculadas con diferentes interval os de muestreo, correspondientes al punto $\mathrm{A} 2$.

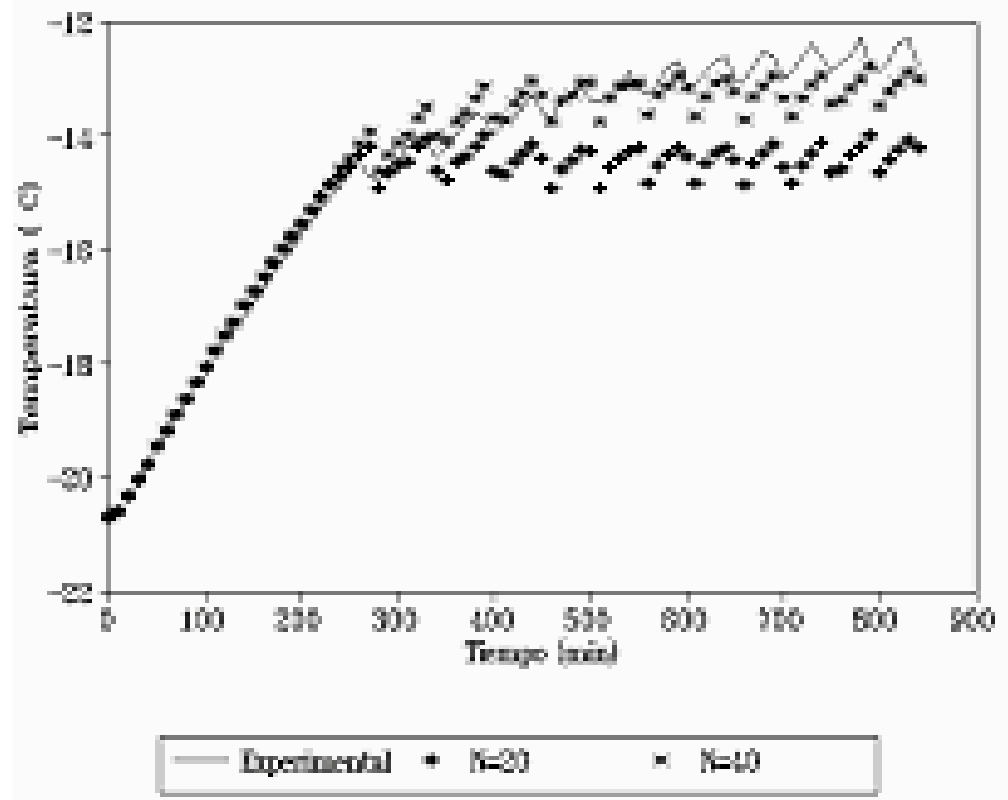

Figura 4.29. Respuestas térmicas, experimental y calculadas con distintos número de coeficientes, del punto $A 2$. 


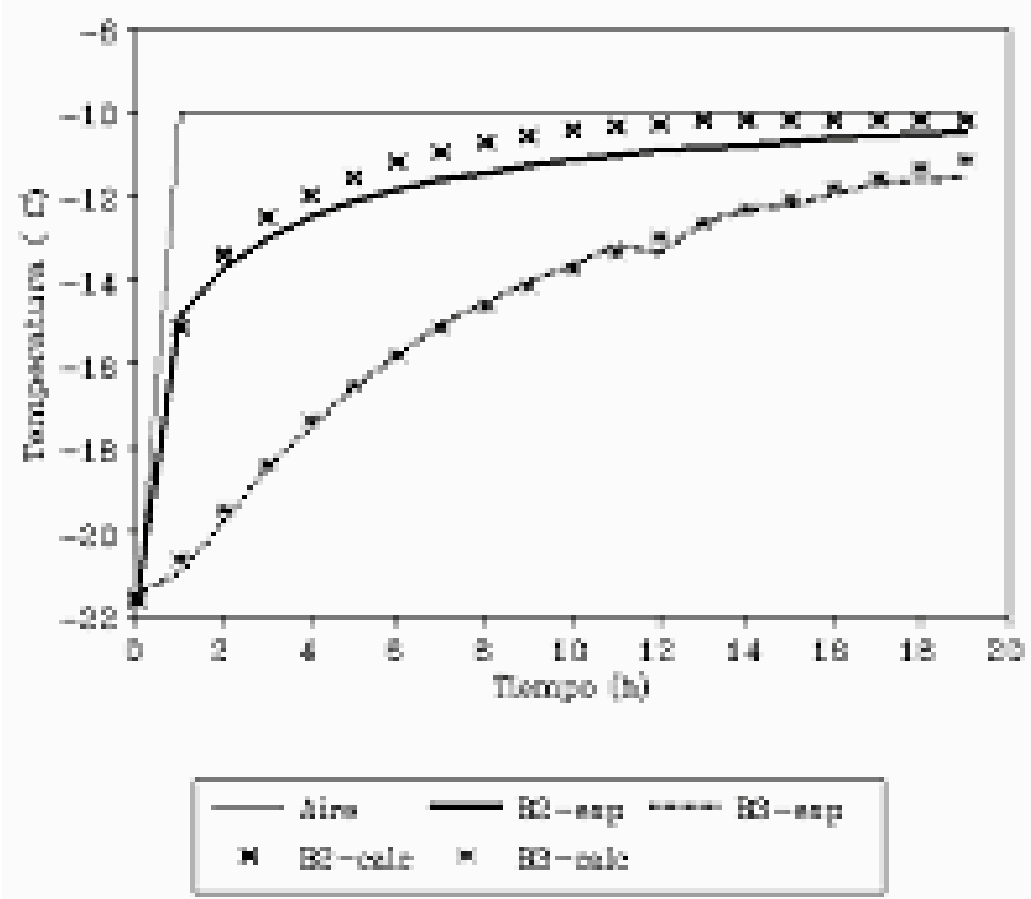

Figura 4.30. Respuestas térmicas, experimental y calculadas de dos puntos representativos de la estiba $B$, frente a una perturbación escalón.

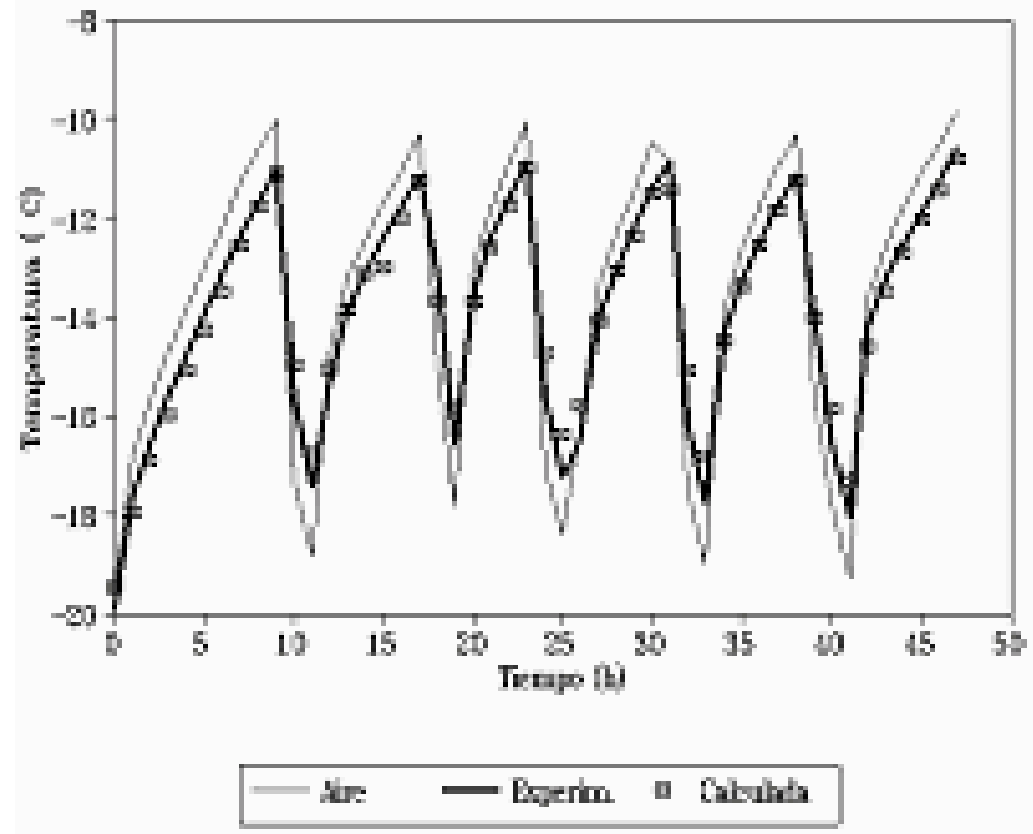

Figura 4.31. Respuestas térmicas, experimental y calculadas del punto B2 frentea una perturbación seno-exponencial. 


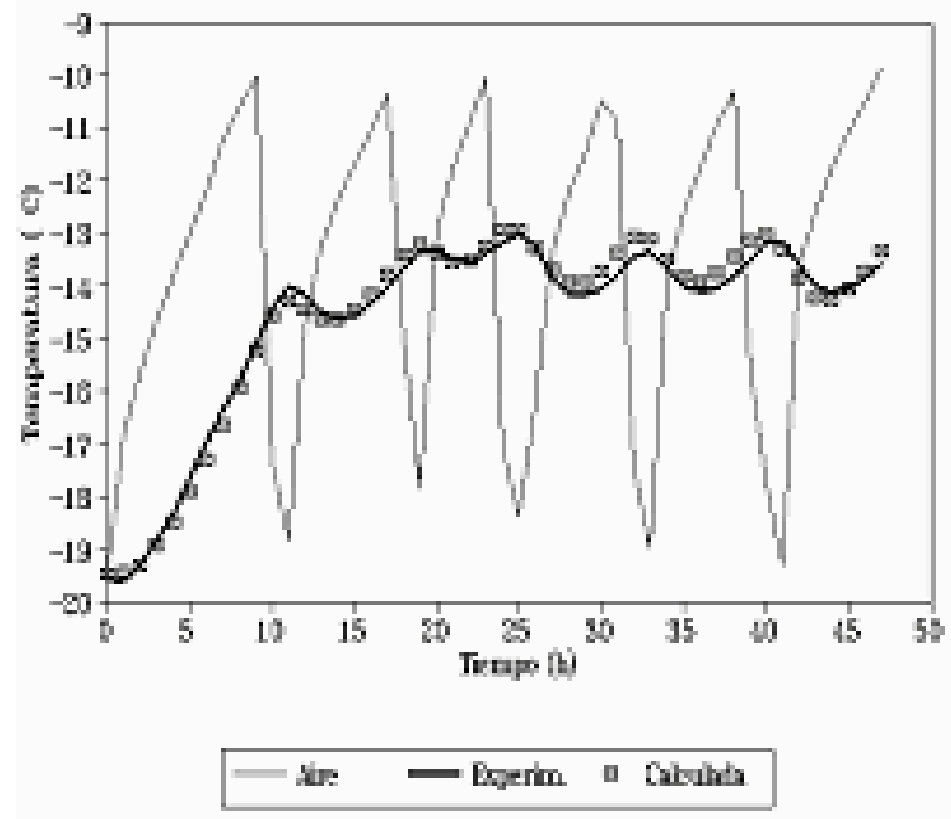

Figura 4.32. Respuestas térmicas, experimental y calculadas del punto B5 frente a una perturbación seno-exponencial.

\subsection{PERDIDA DE CALIDAD DURANTE EL ALMACENAMIENTO}

La calidad de un producto alimenticio es un concepto complejo que incluye consideraciones sobre propiedades sensoriales, composición, características nutritivas, propiedades físicas, crecimiento microbiano, etc. A la vez el concepto de calidad total es un término vago ya que no existe un criterio absoluto de calidad que pueda aplicarse a todos los alimentos, suele evaluarse siguiendo la evolución de un "indicador de calidad". Idealmente el indicador de calidad deberá ser un atributo característico de la condición del alimento; este parámetro debe poder medirse con suficiente precisión y reproducibilidad.

En este trabajo trataremos de puntualizar las variables que provocan el deterioro de los alimentos congelados durante su almacenamiento y distribución. Como punto de partida debemos remarcar que si bien la congelación, efectuada en condiciones controladas, no causa un deterioro importante del alimento (el producto congelado que es descongelado de inmediato casi no puede distinguirse del fresco (Jul, 1984)), tampoco aumenta su calidad razón por la cual la calidad final del alimento luego de su procesamiento dependerá en mayor medida de la calidad 
original de las materia primas. La mayor parte del deterioro se produce durante el almacenamiento. Las pérdidas de calidad debidas al almacenamieno serán relevantes siempre que no sean despreciables frente a las pérdidas que se producen durante la preparación posterior (por ejemplo, en la cocción de verduras se pierde una cantidad de nutrientes muy superior a la pérdida atribuible al almacenamiento).

El deterioro de un alimento está relacionado con diversos cambios, físicos y químicos, que se producen en el mismo. Entre otros podemos mencionar:

Recristalización: Es uno de los cambios físicos más importantes que se producen durante el almacenamiento; consiste en el aumento de tamaño de los cristales grandes a expensas de los cristales más pequeños.

Martino \& Zaritzky (1987, 1988) han estudiado el fenómeno de recristalización en un sistema modelo y en músculo cárneo, obteniendo los parámetros cinéticos que caracterizan el crecimiento del grano cristalino. Estos autores encuentran que las oscilaciones térmicas producidas durante el almacenamiento producen un deterioro mayor que el que se encuentra en el producto mantenido a temperatura constante. El aumento de temperatura provoca además una disminución de la fracción de agua congelada, se funden preferentemente los cristales pequeños, aumentando el tamaño medio de cristales. La recristalización afecta también la desnaturalización de proténas (Wagner \& Añón, 1986).

En cremas heladas Dolan y col. (1985) encuentran que las fluctuaciones de temperatura durante el almacenamiento provocan una disminución de la firmeza (medida como la fuerza requerida para penetrar el centro de una muestra con una cuchara) pero no afecta la cantidad de hielo sentido en el paladar (iciness).

Reacciones con polisacáridos: En salsas cremosas como las que suelen acompañar las comidas preparadas congeladas y en productos de panadería se produce una retrogradación de los gránulos de almidón, lo que provoca la separación de la fase acuosa, el agente espesante pierde uniformidad y el aspecto del alimento se torna desagradable (Ferrero y col., 199 ); este cambio puede 
revertirse por calentamiento.

En jugos de fruta concentrados frente a una historia térmica adversa se produce la pérdida de la turbidez después de reconstituirlo, por insolubilización de la pectina presente por acción de la pectinaestereasa, en algunas ocasiones se producela formación deun gel.

Desnaturalización de proteínas: Consiste en la modificación de la estructura secundaria, terciaria o cuaternaria de la proténa. Se manifiesta de varias maneras (Wagner, 1986): aumento de la susceptibilidad a hidrólisis por enzimas proteolíticas; disminución de la actividad biológica de enzimas; incremento de la viscosidad, incremento de la reactividad química. La velocidad a que se produce cualquiera de los cambios descriptos es función de la temperatura de almacenamiento. En productos cárneos (vacuno, aves, pescados) congelados la desnaturalización proteica del músculo se traduce en una disminución de la capacidad de retención de agua, lo que origina un incremento en el exudado y afecta principalmente la terneza.

Oxidación de lípidos: Consiste en la autooxidación e hidrólisis de la grasa intramuscular presente en los distintos tipos de carnes. Se producen aldehídos, cetonas y ácidos de bajo peso molecular que imparten un olor y sabor a rancio muy desagradable que provoca queel consumidor rechace el producto.

Textura: La textura está vinculada a la calidad estructural del alimento, a la turgencia de las fibras vegetales o a la terneza en el caso de músculo cárneo; los cambio de textura están relacionados a las reacciones que ocurren con polisacáridos o proteínas ya descriptas (Van Arsdel et al. (1969)).

Aspecto: Cualquier cambio en la apariencia respecto el alimento fresco será detectado por el consumidor como desfavorable, aunque no represente una disminución de la calidad nutritiva del mismo, y se reflejará en una menor capacidad de venta. Los más importantes son los cambios de color, ya sea el cambio del color natural de algunos constituyentes (clorofila a pheofitinas: verde brillante que vira a verde oliva, desagradable, se produce en arvejas y espinaca; en carnes rojas se produce oxidación de mioglobina a metamioglobina que imparte un tono 
amarronado indeseable (Lanari \& Zaritzky, 1988)); la pérdida de color hacia el medio que rodea al alimento (en frambuesas y cerezas en almíbar, el color difunde al mismo); o el desarrollo de color desagradable de constituyentes original mente sin color (en aves se produce un color oscuro después de la cocción, en bastones de papa suelen utilizarse antioxidantes para evitar el desarrollo de tonos oscuros).

Quemado por congelación: Consiste en una deshidratación superficial localizada debido a la sublimación del hielo; ya que su presión de vapor es mayor que la del aire circundante. Las áreas afectadas por esta deshidratación son más propensas a que ocurran otros fenómenos como la oxidación de lípidos. Se evita con una envoltura correcta que no permita la migración del vapor de agua.

Valor nutritivo: El cambio más desfavorable que ocurre es la pérdida de vitaminas, especialmente las más lábiles como el ácico ascórbico (vitamina C), tiaminas y riboflavinas (vitaminas B); varios estudios han comprobado que el valor nutritivo de proteínas y lípidos no se ve afectado por la congelación y posterior almacenamiento (Fennema, 1977).

Las vitaminas están presentes en la mayoría de los alimentos y desde el punto de vista nutricional son indispensables en cualquier dieta. Son altamente suceptibles a la degradación química pero generalmente la pérdida de vitaminas atribuible al procesamiento del alimento no se produce durante el almacenamiento sino en otras etapas. Por ejemplo en los vegetales gran parte de la misma (principalmenteácido ascórbico) es causada por el escaldado que se realiza previo a la congelación para evitar el deterioro enzimático; en productos cárneos se pierde vitamina $B$ junto al exudado, típico dela descongelación.

Crecimiento de microorganismos: A pesar de los cuidados que se tengan en la planta de procesamiento en lo que respecta a la higiene, los alimentos son congelados con una determinada población microbiana (obviamente muy por debajo deloslímites de aceptabilidad del producto) que se mantiene parcialmente durante la congelación y posterior almacenamiento, y que comienza a reproducirse cuando el alimento es descongelado. Debajo de $-12^{\circ} \mathrm{C}$ no se produce el crecimiento de ningún microorganismo, pero si la temperatura supera dicho valor, aún manteniéndose por debajo de $0^{\circ} \mathrm{C}$, se observa crecimiento de bacterias psicrófilas 
que si bien no presentan un riesgo para la salud pueden ocasionar cambios de color o textura desfavorables.

\subsubsection{CALCULO DE LA PERDIDA DE CALIDAD}

Como la temperatura y el tiempo son los factores que afectan en mayor medida la pérdida de calidad, es deseable poder predecir mediante ecuaciones los cambios que se producen a partir de la evolución térmica durante el período de almacenamiento.

En general la calidad de un alimento se mide en términos de su vida útil, expresada en días o meses. Dos conceptos se han utilizado para evaluar la aptitud del alimento para ser consumido: HQL (High Quality Life) que se define como el tiempo transcurrido hasta que se produce en el alimento el primer cambio apreciable que suele identificarse con la sigla JND (Just Noticeable Difference) y PSL (Practical Storage Life) que es el período durante el cual el producto es apto para elconsumo. Este último valor es generalmente entre cuatro y cinco veces mayor que el HQL. Cuando se grafican ambos valores en función de la temperatura de almacenamiento en un diagrama semilogarítmico suelen obtenerse líneas rectas. Van Arsdel y col. (1969) presentan una extensa recopilación de estas curvas, realizadas para productos individuales, que globalmente se conocen como curvas TTT (Time-Temperature-Tolerance), en un trabajo posterior Bengtsson y col. (1972) recopilan la información disponible para los distintos grupos de alimentos (únicamente estudios de evaluación sensorial de la calidad) y presentan diagramas de HQL y PSL donde en vez de líneas se observan zonas de validez para los distintos grupos.

Schwimmer y col. (1955) han sido los primeros en desarrollar un modelo de predicción para evaluar el deterioro que se produce a causa de las fluctuaciones de la temperatura de almacenamiento. Estos autores proponen calcular una temperatura efectiva que, por definición, cause el mismo deterioro que algunos modos cíclicos de fluctuación, tales como ondas cuadradas, triangulares y sinusoidales. Para poder aplicar esta teoría debe conocerse la cinética de las reacciones responsables de la pérdida de calidad, y el parámetro $Q_{10}$, que se define como el cociente entre la velocidad de deterioro a $(T+10)$ y la correspondiente a la 
temperatura T.

Heldman \& Lai (1983) desarrollan una expresión para calcular la vida útil remanente luego de almacenar un producto congelado por cierto tiempo a temperatura constante o variable, independientemente del orden de la cinética de deterioro.

Distintas experiencias confirman que el efecto de almacenar los alimentos congelados a diferentes temperaturas es acumulativo (Jul, 1984). Esto también es válido para calcular el deterioro que sufre el alimento cuando para llegar a los centros de consumo es sometido a distintas temperaturas. Esta regla deja de cumplirse cuando las oscilaciones térmicas son de gran amplitud, o cuando sealcanzan temperaturas tales que el crecimiento de microorganismos es apreciable (en este sentido no es lo mismo almacenar un producto un mes a $-3^{\circ} \mathrm{C}$ y luego tres meses a $-20^{\circ} \mathrm{C}$ que la inversa (tres meses a $-20^{\circ} \mathrm{C}$ seguidos de un mes a $-3^{\circ} \mathrm{C}$ ), el recuento microbiano final será mayor en la primera variante). 


\section{NOMENCLATURA}

a Parámetro empírico, ecs. (2.54) y (3.9).

a' Parámetro que caracteriza la forma del cuerpo en la ec. (2.40).

A Superficie del alimento, $\mathrm{m}^{2}$.

A 1 Constante, ec. (2.61).

b Parámetro empírico, ecs. (2.54) y (3.9).

b' Agualigada.

$\mathrm{Bi} \quad$ Número de Biot, definido como ( $\mathrm{h} \mathrm{L} / \mathrm{ko}$ ).

$\mathrm{Bi}^{\prime} \quad$ Número de Biot, definido como $(\mathrm{h} \mathrm{D} / \mathrm{ks})$.

$\mathrm{Bi}^{\prime} 1 \quad$ Número de Biot, promedio entre 2Bi y Bi'.

Bim1 Número de Biot, definido como $\left(h_{1} D / k m\right)$.

Bim2 Número de Biot, definido como ( $\left.\mathrm{h}_{2} \mathrm{D} / \mathrm{km}\right)$.

C Parámetro empírico, ecs. (2.51) y (3.6).

$\mathrm{C}_{1}$ Constante, ec. (2.61).

$\mathrm{C}_{2}$ Constante, ec. (2.61).

$\mathrm{C}_{\mathrm{p}}$ Calor específico o Calor específico aparente, $\mathrm{J} /\left(\mathrm{kg}^{\circ} \mathrm{C}\right)$.

$\mathrm{C}_{\mathrm{po}}$ Calor específico del alimento fresco.

Cps Calor específico del alimento congelado.

D Espesor o Diámetro, $\mathrm{m}$ (o cm cuando se indica específicamente).

Di Parámetro dela ec. (2.21).

Di Parámetro dela ec. (2.21).

Di Parámetro dela ec. (2.21).

e Error porcentual, \%.

- $\quad$ Error porcentual promedio, \%.

e(t) Función entrada o perturbación.

E Altura dela señal escalón.

Ea Energía de activación, J/ ??.

EHTD Número de dimensiones equivalentes.

$E(T) \quad$ Factor definido por la ec. (2.21).

$\mathrm{E}(\mathrm{z}) \quad$ Transformada z dela función e(t).

$\mathrm{F}\left(\mathrm{Bi}^{\prime}\right)$ Factor definido por la ec. (2.22).

Fo Número de Fourier, definido como $\left(\alpha_{0} t / L^{2}\right)$.

$\mathrm{F}(\mathrm{z}) \quad$ Función detransferencia. 
G Producto de los primeros coeficientes correspondientes a las caras $\mathrm{i}, \mathrm{j}, \mathrm{k}, \mathrm{ec}$. (2.22).

h Coeficiente detransferencia calórica, $\mathrm{W} /\left(\mathrm{m}^{2 \circ} \mathrm{C}\right)$.

$\mathrm{h}_{1} \quad$ Coeficiente de transferencia calórica de la cara 1.

h2 Coeficiente detransferencia calórica de la cara 2.

H Ental pía absoluta, J.

- Ental pía específica, J/ kg.

$\mathrm{H}^{*} \quad$ Ental pía volumétrica, $\mathrm{J} / \mathrm{m}^{3}$.

HQL Vida útil, deóptima calidad (High quality life).

k Conductividad térmica, $\mathrm{W} /\left(\mathrm{m}^{\circ} \mathrm{C}\right)$.

ko Conductividad térmica del alimento fresco.

$\mathrm{km} \quad$ Conductividad térmica a la temperatura $\mathrm{Tm}$.

kQ Constante cinética.

$\mathrm{ks} \quad$ Conductividad térmica del alimento congelado.

L Longitud característica: semiespesor o radio, $\mathrm{m}$ (o cm cuando se indica específicamente).

Leq Longitud equivalente, $\mathrm{m}$ (o cm cuando se indica específicamente).

Leq1 Longitud equivalente, según el método de De Michelis \& Calvelo.

Leq2 Longitud equivalente, según el método de Pham.

Leq3 Longitud equivalente, según el método propuesto.

m Parámetro empírico, ecs. (2.48) y (3.6).

n Parámetro empírico, ecs. (2.48) y (3.6).

$\mathrm{N}_{\mathrm{s}} \quad$ Fracción en peso de sólidos.

p Presión, Pa.

$p(t) \quad$ Función perturbación, continua.

$\mathrm{p}^{*}(\mathrm{t}) \quad$ Función muestreada.

pp Parámetro, ec. (2.60).

P Parámetro dela ec. (2.7) y sus modificaciones.

Pk Número de Plank, definido por la ec. (2.12).

PSL Vida útil del alimento, días o meses (Practical Storage Life).

$\mathrm{P}(\mathrm{z}) \quad$ Transformada $z$ dela función $\mathrm{p}(\mathrm{t})$.

q Flujo decalor, $\mathrm{W} / \mathrm{m}^{2}$.

qp Parámetro, ec. (2.60).

Q Calidad. 
Q0 Calidad inicial.

Qf Calidad final.

$r \quad$ Coordenada generalizada de posición, $m$.

rp Parámetro, ec. (2.58).

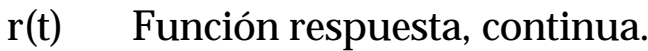

$r^{*}(t) \quad$ Función muestreada.

R Parámetro de la ec. (2.7) y sus modificaciones.

$\mathrm{Rg} \quad$ Constante universal delos gases.

$\mathrm{R}(\mathrm{z})$ Transformada $z$ dela función $\mathrm{r}(\mathrm{t})$.

SP Parámetro, ec. (2.59).

$\mathrm{s}(\mathrm{t}) \quad$ Función salida o respuesta.

Sj Superficiedela cara j, $\mathrm{m}^{2}$.

Ste Número deStefan, definido por la ec. (2.11).

$\mathrm{S}(\mathrm{z}) \quad$ Transformada $z$ dela función $\mathrm{s}(\mathrm{t})$.

t Tiempo, s (min o h cuando se indica específicamente).

tc Tiempo de congelación.

tc1 Tiempo de congelación, asimétrico, cal culado con Leq1.

tc2 Tiempo de congelación, asimétrico, cal culado con Leq2.

tc3 Tiempo de congelación, asimétrico, cal culado con Leq3.

tce Tiempo de cambio de fase.

tcexp Tiempo de congelación, experimental.

tcnum Tiempo de congelación, numérico.

tcpre Tiempo de congelación, predicho.

td Tiempo de descongelación.

tdexp Tiempo de descongelación, experimental.

tdpre Tiempo de descongelación, predicho.

tp $\quad$ Tiempo de preenfriamiento.

tr Tiempo de atemperado.

$\mathrm{T}$ Temperatura, ${ }^{\circ} \mathrm{C}$.

To Temperatura media final.

$\mathrm{Ta} \quad$ Temperatura del medio externo.

$\mathrm{T}_{\mathrm{a} 1}$ Temperatura del medio externo, de la cara 1.

$\mathrm{T}_{\mathrm{a} 2}$ Temperatura del medio externo, de la cara 2.

Taire Temperatura del aire del ambiente de al macenamiento. 
Tc Temperatura del centro térmico.

$\mathrm{Tf}_{\mathrm{f}}$ Temperatura de inicio de cambio de fase.

$T_{\mathrm{fm}}$ Temperatura media de cambio de fase.

$\mathrm{Ti} \quad$ Temperatura inicial.

$\mathrm{Tm}_{\mathrm{m}} \quad$ Temperatura promedio de $\mathrm{T}_{\mathrm{f}} \mathrm{y} \mathrm{T}_{\mathrm{a}}$.

Tme Temperatura media entál pica.

Tref Temperatura de referencia.

Tw Temperatura de congelación del agua pura.

$\mathrm{T}^{*} \quad$ Temperatura ficticia (modelo numérico multidimensional).

$T^{* *}$ Temperatura ficticia (modelo numérico multidimensional).

U Temperatura adimensional.

$\mathrm{v} \quad$ Velocidad, $\mathrm{m} / \mathrm{s}$.

$\mathrm{V}$ Volumen, $\mathrm{m}^{3}$.

w Fracción másica de hielo.

Wa Fracción másica de agua líquida.

Wt Fracción másica de tejido seco.

W Masa del alimento, $\mathrm{kg}$.

$x, y, z$ Coordenadas de posición, modelo numérico.

XP Parámetro, ec. (2.57).

$X \quad$ Variable adimensional, definida por las ecs. (2.48) y (3.6).

Y Contenido de agua, $\mathrm{kg}$ agual (kg masa total).

$Y_{0} \quad$ Contenido inicial de agua.

$Y_{n} \quad$ Fracción deagua sin congelar.

z Variable compleja.

$\alpha \quad$ Difusividad térmica, $\mathrm{m}^{2} / \mathrm{s}$.

$\alpha_{0} \quad$ Difusividad térmica del alimento fresco.

$\alpha_{s} \quad$ Difusividad térmica del alimento congelado.

$\triangle \quad$ Intervalo de muestreo.

$\Delta \mathrm{H} \quad$ Diferencia de ental pía absoluta, J.

$\Delta_{-} \quad$ Diferencia de ental pía específica, J/ kg.

$\Delta \mathrm{H}^{*} \quad$ Diferencia de ental pía volumétrica, $\mathrm{J} / \mathrm{m}^{3}$.

$\Delta r \quad$ Incremento de espacio del modelo numérico.

$\Delta \mathrm{t} \quad$ Incremento de tiempo del modelo numérico. 
$\Delta \mathrm{T} \quad$ Diferencia de temperaturas.

$\Delta T_{1} \quad$ Diferencia de temperaturas, ec. (2.2).

$\Delta T_{2}$ Diferencia de temperaturas, ec. (2.2).

$\triangle \mathrm{Tm}$ Diferencia de temperaturas media logarítmica.

$\lambda_{1} \quad$ Primera raíz de la ec. trascendental $\lambda_{n} L \operatorname{tg}\left(\lambda_{n} L\right)=B i$.

$\lambda_{f} \quad$ Calor latente de fusión, $\mathrm{J} / \mathrm{kg}$.

$\lambda_{\mathrm{f}, \mathrm{e}}^{*} \quad$ Calor latente de fusión efectivo volumétrico, $\mathrm{J} / \mathrm{m}^{3}$.

$\rho$ Densidad, $\mathrm{kg} / \mathrm{m}^{3}$.

Po Densidad del alimento fresco.

$\rho_{s} \quad$ Densidad del alimento congelado.

$\tau$ Tensor esfuerzo viscoso, $\mathrm{N} / \mathrm{m}^{2}$.

$\nabla \quad$ Operador divergencia.

Subíndices

h Indica asimetría originada en distintos coeficientes de transferencia calórica.

i Evaluado en la posición ((i-1) $\Delta r$ ) en el método de diferencias finitas.

$\mathrm{T}$ Indica asimetría originada en distintas temperaturas externas.

Supraíndices

$\mathrm{n} \quad$ Evaluado al tiempo $\mathrm{n}(\Delta \mathrm{t})$ en el método de diferencias finitas. 
Bibliografía

\section{REFEREN CIAS BIBLIOGRAFICAS}

ASHRAE Handbook (1985) Fundamentals, A merican Society of Heating, Refrigerating and $A$ ir Conditioning Enginers Chapters 23 and 26.

Bailey, C., James, S.J., Kitchell, A.G . \& Hudson, W.R. (1974) Air water and vacuum thawing of frozen pork legs. J Sci Food A gric 25:81.

Bazán, H.C. \& Mascheroni, R.H. (1984) Heat transfer with simultaneous change of phase in freezing boned mutton. Lat A $\mathrm{m} J \mathrm{H}$ eat M ass Transf 8:55.

Barakat S.A. (1987) Experimental determination of the z-transfer function coefficients for houses, ASH RA E Trans 93, Part 1:146.

Bengtsson, N., Liljemark, A., Olsson, P. \& Nilsson B. (1972) An attempt to systemize time-temperature-tolerance (TTT) data as a basis for the development of timetemperature indicators. Bull IIR Annexe 2:303.

Bonacina, C. \& Comini, G . (1971) On a numerical method for the solution of the unsteady state heat conduction equation with temperature dependent parameters. Proc 13th Int Cong Refrig 2:329.

Bonacina, C. \& Comini, G. (1973) On the solution of he nonlinear heat conduction equations by numerical methods. Int J $\mathrm{H}$ eat $\mathrm{M}$ ass Transfer 16:581.

Calvelo, A . (1981) Recent studies on meat freezing in Developments in Meat Science- 2 (Ed. R. Lawrie) A pplied Science Publishers Ltd, London, 125.

Carslaw, H.S. \& Jaeger, J.C. (1959) Conduction of heat in solids. Oxford University Press, 2nd Edition, Oxford.

Ceylan H. T. (1987) Transient heat transfer in long cylinders with time dependent surface temperature. ASH RAE Trans 93, Part 2:768.

Cleland, A.C. (1990) Food refrigeration processes. Analysis, design and simulation. Elsevier A pplied Science Publishers Ltd, London.

Cleland, A.C. \& Earle, R.L. (1977) A comparison of analytical and numerical methods for predicting freezing times of foods. J Food Sci 42:1390.

Cleland, A.C. \& Earle, R.L. (1979a) A comparison of methods for predicting the freezing times of cylindrical and spherical foodstuffs. J Food Sci 44:958.

Cleland, A.C. \& Earle, R.L. (1979b) Prediction of freezing times for foods in rectangular packages. J Food Sci 44:964.

Cleland, A.C., Earle, R.L. \& Cleland, D.J. (1982) The effect of freezing rate on the accuracy of numerical freezing time predictions. Int J R efrig 5:294.

Cleland, A.C. \& Earle, R.L. (1984a) Assessment of freezing time prediction 
Bibliografía

methods. J Food Sci 49:1034.

Cleland, A.C. \& Earle, R.L. (1984b) Freezing time predictions for different final product temperatures.J Food Sci 49:1230.

Cleland, D.J. \& Cleland, A.C. (1991) An alternating direction, implicit finite difference scheme for heat conduction with phase change in finite cylinders. Proc 18th Int Congress Refrig, Montreal, paginas.

Cleland, D.J., Cleland, A.C., Earle, R.L. \& Byrne, S.J. (1986) Prediction of thawing times for foods of simple shape. Int J R efrig 9:220.

Creed, P.G . \& James, S.J. (1981) Predicting thawing times of frozen boneless beef blocks. Int J Refrig 4:355.

Creed, P.G . \& James, S.J . (1983) The freezing times of liver in a vertical plate freezer. Proc 16th Int Congress Refrig, París, IV :145.

Creed, P.G . \& James, S.J. (1985) Heat transfer during the freezing of liver in a plate freezer.J Food Sci 50:285.

Crepey, J.R. \& Becel, P. (1978) Etude experimentale de certaines methodes de decongelation apliquées au thon et a la sardine. Comissions C2, D 1 and D2; IIR, Budapest,

De Michelis, A. \& Calvelo, A. (1982) Mathematical models for nonsymmetric freezing of beef.J Food Sci 47:1211.

De Michelis, A . \& Calvelo, A. (1983) Freezing time predictions for bricks adn cylindrical-shaped foods. J Food Sci 48:909.

De Michelis, A., Mascheroni, R.H. \& Calvelo, A. (1985) Estimación de tiempos de congelación de productos cárneos. La A limentación Latinoamericana 19:52.

D.I.E.P.B.A. (1991a) Bases Informativas $N^{\circ} 6$ : Pescados. Aves y Huevos. Dirección de Investigaciones Económicas de la Prov. de Buenos Aires, Ministerio de Economía, La Plata.

D.I.E.P.B.A. (1991b) Bases Informativas Nº7: Industria Frigorífica. Dirección de Investigaciones Económicas de la Prov. de Buenos Aires, Ministerio de Economía, La Plata.

D olan (1985)

Fennema (1977)

Flechtenmacher, W. (1983) Measurement of heat transfer in thawing of fish fillet block. Part II: Thawing of mackerel fillets in running water. Int Zeits Lebens Technol \& Verfahr 34:362.

Flores, E.S. \& M ascheroni, R.H . (1983) Theoretical and experimental study 
Bibliografía

of thawing of frozen food blocks by aspersion with water. Lat A m J H eat M ass Transf 7:263.

Flores, E.S. \& M ascheroni, R.H . (1984) Tiempos de congelación de bloques decarne picada. CIDCA, Publicación interna Nº39.

Flores, E.S., Bazán, H ., De M ichelis, A . \& M ascheroni, R.H. (1993) Thawing time of blocks of boneless or minced meats. Measure and prediction for different types of equipments. Lat A m A ppl Res 23:79.

Frederick, D. \& G rief, R. (1985) A method for the solution of heat transfer problems with a change of phase. J H eat Transf 107:520.

Hayakawa, K., Scott, K .R. \& Succar, J. (1986) Theoretical and semitheoretical methods for estimating freezing or thawing time. A SH RA E Trans Part 2B 91:371.

Heldman, D.R. (1990) Simulation of temperature dependent quality deterioration in frozen foods. J Food Eng 11:43.

Heldman, D.R. \& Lai, D. (1983) A model for prediction of shelf life for frozen foods. Proc 16th Int Congress Refrig, Paris, 417.

Hense, H. \& Kieckbusch, T.G. (1991) Congelamento de cacao: I-Resultados experimentais. A ctas IV Congreso Latinoamericano de Transferencia de Calor y $M$ ateria.

Hung, Y.C. \& Thompson, D.R. (1983) Freezing time prediction for slab shape foodstuffs by an improved analytical method.J Food Sci 48:555.

IIR (1972) Recommendations for the processing and handling of frozen foods, International Institute of Refrigeration, 2nd Edition, París.

Ilicali, C. (1989) A simplified analytical model for thawing time calculation in foods. J Food Sci 54:1031.

Ilicali, C. \& Saglam, N. (1987) A simplified analytical model for freezing timecalculation in foods. J Food Proc Eng 9:299.

James, S.J., Bailey, C. \& O no, S. (1976) Determination of freezing and thawing times in the centre of blocks of meat by measurement of surface temperature. J Food Technol 11:505.

James, S.J., Creed, P.G . \& Roberts, T.A . (1977) Air thawing of beef quarters. J Sci Food A gric 28:1109.

James, S.J. \& Bailey, C. (1980) Air and vacuum thawing of unwrapped boneless meat blocks. Refrig and $\mathrm{A}$ ir Condit 83:75.

Joshi, C. \& Tao, L. (1974) A numerical method of simulating the axisymmetrical freezing of food systems. J Food Sci 39:623.

Jul, M . (1984) Quality of frozen foods. 
Bibliografía

Labuza, T.P. (1982) Shelf-life dating of foods. Food and N utrition Press Inc.

Lanari, M .C. \& Zaritzky, N .E. (198) J Food Sci

Lees, M. (1966) A linear three level difference scheme for quasi linear parabolic equations. M aths Comput 20:516.

Levenspiel, 0. (1976) Ingeniería de las reacciones químicas. Ed. Reverté, Buenos Aires.

Levy, F.L. (1958) Calculating freezinf time of fish in airblast freezers. J Refrig 1:55.

Levy, F.L. (1983) Calculating the thawing time of meat. Zeits Lebens Technol 34:691.

Luyben, (1973) Process modeling, simulation and control for chemical engineers. M c Graw - Hill Kogakusha Ltd, Caps. 9 y 14.

M annapperuma, J.D. \& Singh, R.P. (1988a) Prediction of freezing and thawing times of foods using a numerical method based on enthalpy transformation. J. Food Sci. 53:626.

Mannapperuma, J.D. \& Singh, R.P. (1988b) Thawing of frozen foods in humid air. Int J Refrig 7:123.

Martino, M.N. \& Zaritzky, N.E. (1987) Effects of temperature on recrystallization in polycrystallineice. Sci A liments 7:147.

M artino, M .N . \& Zaritzky, N.E. (1988) Ice crystal size modifications during frozen beef storage. J Food Sci 53:1631.

M ascheroni, R.H . (1977) Transferencia de calor con simultáneo cambio de faseen productos cárneos. Tesis doctoral, Universidad Nacional de La Plata.

M ascheroni, R.H . (1982) The utilization of numerical methods for solution of the heat balance during the thawing of meat blocks under industrial conditions. Lat A $\mathrm{m} J \mathrm{H}$ eat $\mathrm{M}$ ass Transf 6:13.

Mascheroni, R.H . \& Calvelo, A. (1978) Modelo de descenso crioscópico en tejidos cárneos. La A limentación Latinoamericana 12:34.

Mascheroni, R.H . \& Calvelo, A . (1980) Relationship between heat transfer parameters and the characteristic damage variables for the freezing of beef. M eat Sci 4:267.

M ascheroni, R.H . \& Calvelo, A. (1982) A simplified model for freezing time calculations in foods. J Food Sci 47:1201.

Mascheroni, R.H., Ottino, J. \& Calvelo, A. (1977) A model for the thermal conductivity of frozen meat. M eat Sci 1:235. 
M ascheroni R. H., Sanz P. D . \& D omínguez M . (1987) A new way to predict thermal histories in multidimensional heat conduction: the z-transfer function method. Int Comm H eat M ass Trans 14:81.

Mattarolo, L. (1990) Refrigeration and food processing to ensure the nutrition of the growing world population. Progress in the science and technology of refrigeration in food engineering IIR Commissions B2, C2, D1, D2/ 3:43.

Mitalas, G.P. (1978) Comments on the z-transfer function method for calculating heat transfer in buildings, A SH RA E Trans 84, Part 1:667.

M oleeratanond, W., Kramer, A ., A shby, B.H., Bailey, W.A . \& Bennett, A.H. (1979) Effect of temperature fluctuations on energy consumption and quality changes of palletized foods in frozen storage. A SH RAE Trans 85, Part 2:56.

Nagaoka, J., Takaji, S. \& Hotani, S. (1955) Experiments on the freezing of fish, by air-blast freezer. J Tokio U niv Fish 42:65.

Pham, Q.T. (1984) An extension to Plank's equation for predicting freezing times for foodstuffs of simple shapes. Int J Refrig 7:377.

Pham, Q.T. (1985) A fast, unconditionally stable finite- difference method for heat conduction with phase change. Int J H eat M ass Transf 28:2079.

Pham, Q.T. (1986) Simplified equation for predicting the freezing time of foodstuffs. J Food Technol 21:209.

Pham, Q.T. (1987) A converging front model for the asymmetric freezing of slab shaped foods: a simplified graphical method. Int J Refrig 10:357.

Pham, Q.T. \& Willix, J.(1990) Effect of Biot number and freezing rate on accuracy of some food freezing time prediction methods. J Food Sci 55:1429.

Plank, R. (1963) El empleo del frío en la industria de la refrigeración. Ed. Reverté, Barcelona.

PROMEX (1992a) Situación y perspectivas del mercado nacional e internacional. Frutas y hortalizas procesadas. Proyecto de promoción de exportaciones de productos de base agrícola no tradicional, Secretaría de Agricultura, Ganadería y Pesca, Argentina.

PROM EX (1992b) Argentina, a vegetable garden for the world. Proyecto de promoción de exportaciones de base agrícola no tradicional, Secretaría de Agricultura, Ganadería y Pesca, Argentina.

Ramaswamy, H.S. \& Tung, M.A. (1984) A review on predicting freezing times of foods. J Food Proc Eng 7:169.

Rubiolo de Reinick, A. \& Schwartzberg, H. (1986) Predicting temperature 
vs. time behavior during the freezing and thawing of rectangular foods. Biotech Progress 2:164.

Sanz, P.D . (1984) A nálisis de las funciones de transferencia para la resolución de problemas de transmisión de calor en régimen variable en cámaras adiabáticas y de régimen controlado, Tesis doctoral, Universidad Complutense, Madrid.

Sanz, P.D., Domínguez, M . \& M ascheroni, R.H . (1987) Thermophysical properties of meat products. General bibliography and experimental data. Trans ASAE 30:283.

Sanz, P.D., Domínguez, M . \& Mascheroni, R.H . (1989) Equations for the prediction of thermophysical properties of meat products. Latin A m A ppl Res 19:155.

Sanz, P.D., Mascheroni, R.H., Domínguez, M. \& García de Vinuesa, S. (1986) Time-temperature prediction curves of foodstuffs by means of the z-transfer function method, Int J R efrig 9:89.

Sastry, S.K. (1984) Freezing time prediction: an enthalpy based approach. J Food Sci 49:1121.

Schwartzberg, H.G. (1976) Effective heat capacities for the freezing and thawing of food. J Food Sci 41:152.

Schwartzberg, H.G., Rosenau, J.R. \& H aight, J.R. (1977) The prediction of freezing and thawing temperature vs. time behavior through the use of effective heat capacity equations, in F reezing, Frozen Storage and Freeze D rying, IIR, París: 311.

\section{Schwimmer}

Seem, J.E., Klein, S.A ., Beckman, W.A. \& M itchell, J.W. (1989a) Transfer functions for efficient calculation of multidimensional transient heat transfer, ASM E J H eat Trans 111:5.

Seem, J.E., Klein, S.A., Beckman, W.A. \& Mitchell, J.W. (1989b) Comprehensive room transfer functions for efficient calculation of the transient heat transfer processes in buildings, A SM E J H eat Trans 111:264.

Singh, R.K. \& Lund, D.B. (1984) Mathematical modelling of heat and moisture transfer-related properties of intermediate moisture apples. J Food Proc Pres 8:191.

Singh, R.P. \& Wang, C.Y. (1977) Quality of frozen foods - a review. J Food Proc Eng 1:97.

Stephenson, D.G. \& Mitalas, G.P. (1971) Calculation of heat conduction transfer functions for multi-layer slabs, A SH RA E Trans 77, Part 2:117.

Succar, J. \& Hayakawa, K. (1984) Parametric analysis for predicting freezing 
Bibliografía

time of infinitely slab shaped food. J Food Sci 49:468.

Tocci, A . \& M ascheroni, R.H . (1993) Freezing times of meat balls.falta

Uno, J. \& H ayakawa, K. (1979) Nonsymmetric heat conduction in an infinite slab.J Food Sci 44:396.

V an Arsdel, W.B., Copley, M .T \& \& O Ison, R.L. (1969) Quality and stability of frozen foods. John Wiley \& Sons, New York.

Vanichseni, S. (1971) Thawing of frozen lamb shoulders. M IRINZ Report N ${ }^{\circ}$ 233, Hamilton, New Zealand.

Wagner, J.R. (1986) Desnaturalización de proteínas de músculo bovino durante la congelación y el almacenamiento en estado congelado. Tesis doctoral, Universidad Nacional de La Plata.

Wagner, J.R. \& Añón, M.C. (1986) Effect of frozen storage on protein denaturation in bovine muscle. I.Myofibrillar ATPase activity and differential scanning calorimetric studies. J Food Technol 21:9.

Wang, D.Q. \& Kolbe, E. (1987) Measurement and prediction of freezing times of vacuum canned Pacific shrimp. Int J Refrig 10:18.

Wilkinson, (1990) SYSTAT

Wilson, H .A. \& Singh, R.P. (1987) Numerical simulation of individual quick freezing of spherical particles. Int J R efrig 10:149.

Zaritzky, N.E. (1982) Mathematical simulation of the thermal behavior of frozen meat during its storage and distribution.J Food Eng 6:15.

Zuritz, C. \& Singh, R. (1985) Modelling temperature fluctuations in stored frozen foods. Int J Refrig 8:289. 